\title{
TRY plant trait database - enhanced coverage and open access
}

\author{
Correspondence \\ Jens Kattge, Max Planck Institute for \\ Biogeochemistry, Hans Knöll Str. 10, 07745 \\ Jena, Germany. \\ Email: jkattge@bgc-jena.mpg.de

\section{Funding information} \\ Max Planck Institute for Biogeochemistry; \\ Max Planck Society; German Centre for \\ Integrative Biodiversity Research (iDiv) \\ Halle-Jena-Leipzig; International Programme \\ of Biodiversity Science (DIVERSITAS); \\ International Geosphere-Biosphere \\ Programme (IGBP); Future Earth; French \\ Foundation for Biodiversity Research (FRB); \\ GIS 'Climat, Environnement et Société' \\ France; UK Natural Environment Research \\ Council (NERC); AXA Research Fund
}

\begin{abstract}
Plant traits-the morphological, anatomical, physiological, biochemical and phenological characteristics of plants-determine how plants respond to environmental factors, affect other trophic levels, and influence ecosystem properties and their benefits and detriments to people. Plant trait data thus represent the basis for a vast area of research spanning from evolutionary biology, community and functional ecology, to biodiversity conservation, ecosystem and landscape management, restoration, biogeography and earth system modelling. Since its foundation in 2007, the TRY database of plant traits has grown continuously. It now provides unprecedented data coverage under an open access data policy and is the main plant trait database used by the research community worldwide. Increasingly, the TRY database also supports new frontiers of trait-based plant research, including the identification of data gaps and the subsequent mobilization or measurement of new data. To support this development, in this article we evaluate the extent of the trait data compiled in TRY and analyse emerging patterns of data coverage and representativeness. Best species coverage is achieved for categorical traits-almost complete coverage for 'plant growth form'. However, most traits relevant for ecology and vegetation modelling are characterized by continuous intraspecific variation and trait-environmental relationships. These traits have to be measured on individual plants in their respective environment. Despite unprecedented data coverage, we observe a humbling lack of completeness and representativeness of these continuous traits in many aspects. We, therefore, conclude that reducing data gaps and biases in the TRY database remains a key challenge and requires a coordinated approach to data mobilization and trait measurements. This can only be achieved in collaboration with other initiatives.
\end{abstract}

\section{KEYWORDS}

data coverage, data integration, data representativeness, functional diversity, plant traits, TRY plant trait database 


\section{1 | INTRODUCTION}

Plant traits-the morphological, anatomical, physiological, biochemical and phenological characteristics of plants measurable at the individual plant level (Violle et al., 2007)-reflect the outcome of evolutionary and community assembly processes responding to abiotic and biotic environmental constraints (Valladares, Gianoli, \& Gomez, 2007). Traits and trait syndromes (recurrent coordinated expressions of multiple traits) determine how plants perform and respond to environmental factors (Grime, 1974; Wright et al., 2017), affect other trophic levels (Lavorel et al., 2013; Loranger et al., 2012, 2013), and provide a link from species richness to functional diversity, which influences ecosystem properties and derived benefits and detriments to people (Aerts \& Chapin, 2000; Díaz et al., 2004, 2007; Garnier \& Navas, 2012; Grime, 2001, 2006; Lavorel et al., 2015; Lavorel \& Garnier, 2002). In the context of the Global Earth Observation Biodiversity Observation Network (GEO BON) species traits are considered an Essential Biodiversity Variable to inform policy about biodiversity change (Kissling et al., 2018; Pereira et al., 2013). A focus on traits and trait syndromes, therefore, provides a crucial basis for quantitative and predictive ecology, ecologically informed landscape conservation and the global change sciencepolicy interface (Díaz et al., 2016; McGill, Enquist, Weiher, \& Westoby, 2006; Westoby \& Wright, 2006). To fully realize this potential, plant trait data not only need to be available and accessible in appropriate quantity and quality but also representative for the scales of inference and research questions (König et al., 2019). Here we analyse where the TRY plant trait database stands with respect to coverage and representativeness after 12 years of operation. We further review the mechanisms and emergent dynamics helping to increase both.

\section{1 | A global database of plant traits-A brief history}

Before the foundation of TRY in 2007, several research groups had already developed major plant trait databases with remarkable success, e.g. the Ecological Flora of the British Islands
(Fitter \& Peat, 1994), the Seed Information Database (Royal Botanical Gardens KEW, 2008), BIOPOP (Poschlod, Kleyer, Jackel, Dannemann, \& Tackenberg, 2003), GLOPNET (Wright et al., 2004), BiolFlor (Klotz, Kühn, \& Durka, 2002, 2017), LEDA (Kleyer et al., 2008), BROT (Paula et al., 2009), USDA PLANTSdata (Green, 2009) and BRIDGE (Baraloto, Timothy Paine, Patino, et al., 2010). However, these databases were either focused on particular regions (BiolFlor, LEDA, BIOPOP, BROT, USDA Plants, Ecological Flora of the British Islands, BRIDGE) or specific traits (GLOPNET, SID). A 'database of databases' was in discussion for some time, but it had been impossible to secure long-term funding for such a project. Finally, at a joint workshop of the International Geosphere-Biosphere Program (IGBP) and DIVERSITAS, the TRY database (TRY-not an acronym, rather a statement of sentiment; https://www.try-db.org; Kattge et al., 2011) was proposed with the explicit assignment to improve the availability and accessibility of plant trait data for ecology and earth system sciences. The Max Planck Institute for Biogeochemistry (MPI-BGC) offered to host the database and the different groups joined forces for this community-driven program. Two factors were key to the success of TRY: the support and trust of leaders in the field of functional plant ecology submitting large databases and the long-term funding by the Max Planck Society, the MPI-BGC and the German Centre for Integrative Biodiversity Research (iDiv) Halle-Jena-Leipzig, which has enabled the continuous development of the TRY database.

At the time of the foundation of TRY, data sharing was not yet a common practice in ecology (Kattge et al., 2011; Reichman, Jones, \& Schildhauer, 2011). This was an important obstacle for scientific progress. The first important step of the initiative was, therefore, to jointly develop a data sharing policy. This was based on permission of data set owners and a 'give-and-take' system: to keep the TRY database growing, the right to request data was coupled to data contribution. Exceptions were data requests for vegetation modelling projects, as modellers typically do not own plant trait data. At an open workshop in 2013, the members decided to offer the opportunity to make data publicly available and trait data contribution was no longer a requirement for data access. In 2014, this decision was implemented in the TRY Data Portal and was immediately followed by an 'explosion' of the number of data requests (Figure 1a): TRY
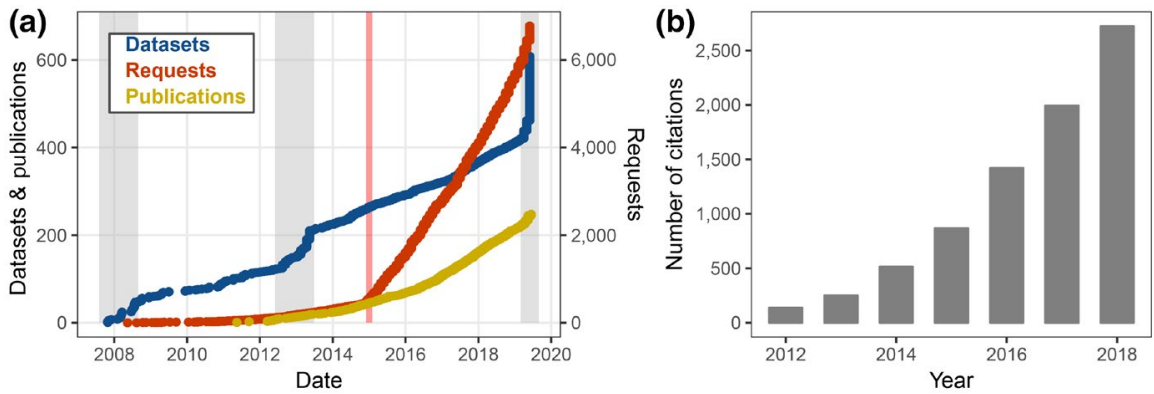

FIGURE 1 TRY performance statistics, status 1 July 2019. (a) Cumulative numbers of data sets and publications (left axis) and data requests (right axis); light grey vertical bars indicate calls for data contribution; the red vertical bar indicates the date of opening TRY to the public. (b) Number of citations for publications using trait data via TRY (Google Scholar) 
started to serve more than 1,000 requests per year, so that as of July 2019, about 700 million trait records accompanied by 3 billion ancillary data have been released for 7,000 requests, submitted by more than 5,000 registered users. Since 2019, the TRY database is open access under a Creative Commons Attribution license (CC BY 4.0, https://creativecommons.org/licenses/by/4.0): anyone can use and redistribute data received via TRY under the only condition of appropriate citation of the TRY database and the references of contributing data sets. Restriction of data access now is the exception and limited to 2(+2) years, after which the data sets become public.
Since 2014, the TRY Data Portal (https://www.try-db.org/Try Web/dp.php) has become the central access point of the TRY database: the portal organizes data uploads, searches and requests, and enables interaction between data contributors, management and users. The portal provides an account for each data set custodian (the individual who directly contributed the data set), which provides precise bookkeeping about the use of his or her trait data via TRY. The TRY Data Portal also provides a link to the TRY File Archive (https://www.try-db.org/TryWeb/Data.php), which offers climate and soil data for TRY measurement sites, standardized

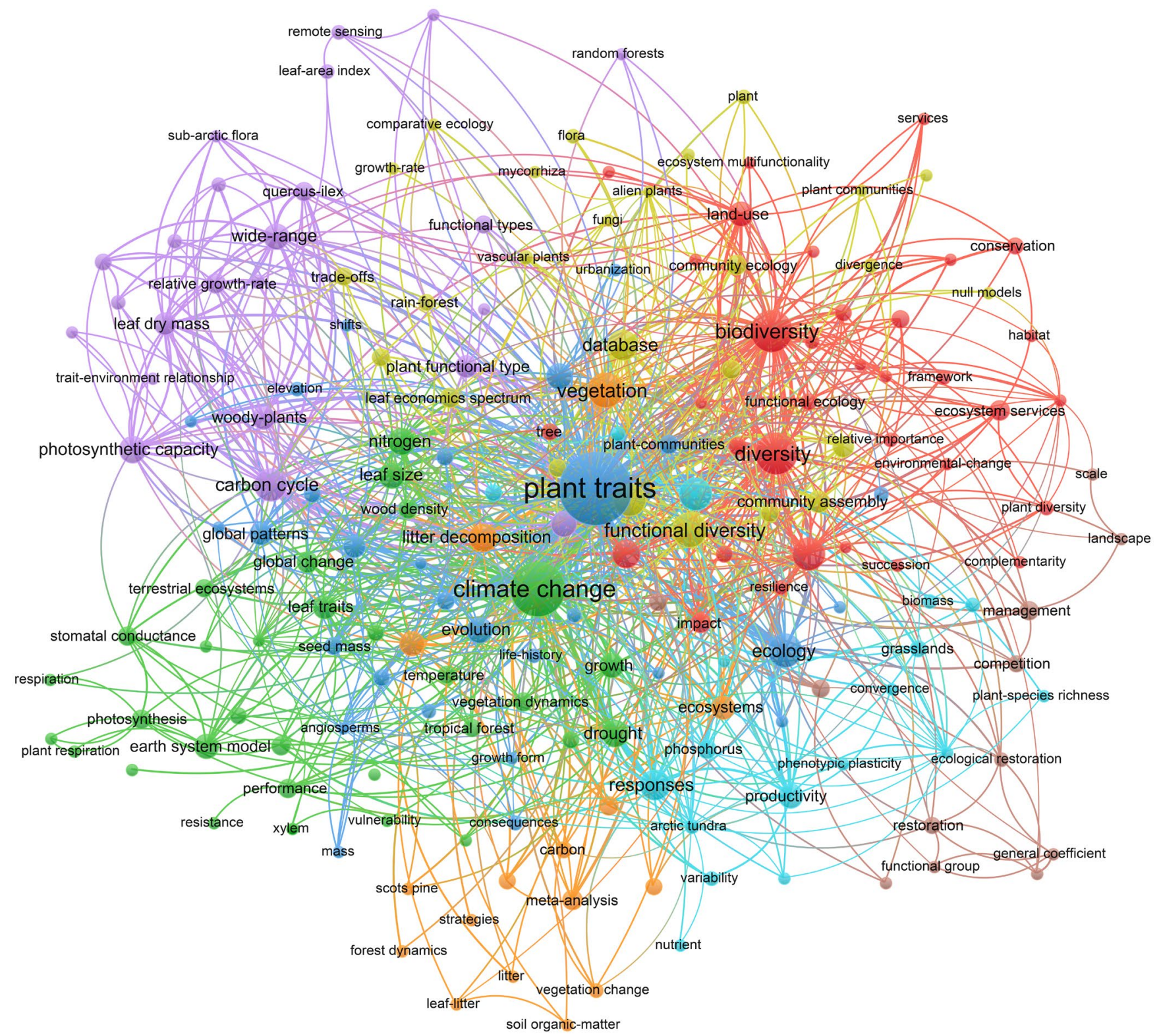

FIGURE 2 Cluster analysis of keywords from peer-reviewed publications using plant trait data via TRY. The size of the circles and letters indicates the frequency of the keywords, colours indicate the eight clusters around the central keywords (from largest to smallest cluster): biodiversity (red), climate change (dark green), plant traits (dark blue), functional diversity (light green), carbon cycle (violet), community (light blue), vegetation (orange) and environmental filtering (brown). The analysis is based on 190 publications with DOIs compiled by ISI Web of Science (https://clarivate.com/products/web-of-science). The analysis was performed with VOSviewer version 1.6.11 (https://www.vosvi ewer.com) using the default settings and only minor editing of selected terms. The clustering technique used by VOSviewer is discussed by Waltman, van Eck, and Noyons (2010). Due to limited space not all central keywords of small clusters are displayed. Material to display the results in detail using the VOSviewer software is provided in the Supporting Information 
categorical traits relevant to attribute species to plant functional types (PFTs), and provides the opportunity to publish plant trait data sets and receive a DOI.

Trait data via TRY contributed to at least 250 scientific applications and publications (Figure 1a), among these 202 peer-reviewed publications in 83 different scientific journals, covering a broad range of topics, from 'Landscape and Urban planning' to 'Geoscientific Model Development'. Twenty publications were directly related to vegetation model development, while 230 were empirical studies. A cluster analysis of keywords from the peer-reviewed publications shows eight clusters around the central keywords biodiversity, climate change, plant traits, functional diversity, carbon cycle, community, vegetation and environmental filtering (Figure 2). Citations of publications using trait data via TRY have increased exponentially, leading to about 10,000 citations and an h-factor of 46 for the TRY database (Figure $1 \mathrm{~b}$ ).

During 12 years of development, versions 1-5 of the TRY database have been released with an increasing number of contributed data sets and trait records (Tables 1 and 2; Figure 1a). Currently, TRY is working on version 6. As of July 2019, the TRY database comprised 588 data sets from 765 data contributors (Table A1). The dynamics of the number of data sets in TRY indicates an increasing success of calls to the scientific community for data

TABLE 1 TRY database versions

\begin{tabular}{|c|c|c|c|}
\hline Version & $\begin{array}{l}\text { Data } \\
\text { acquisition and } \\
\text { import }\end{array}$ & Data release & Status \\
\hline 1 & $\begin{array}{l}\text { October } 2007- \\
\text { July } 2009\end{array}$ & $\begin{array}{l}\text { October } 2008- \\
\text { April } 2011\end{array}$ & $\begin{array}{l}\text { Restricted, } \\
\text { give-and-take }\end{array}$ \\
\hline 2 & $\begin{array}{l}\text { July 2009- } \\
\text { April } 2011\end{array}$ & $\begin{array}{l}\text { April 2011- } \\
\text { December } 2014\end{array}$ & $\begin{array}{l}\text { Restricted, } \\
\text { give-and-take }\end{array}$ \\
\hline 3 & $\begin{array}{r}\text { April 2011- } \\
\text { April } 2014\end{array}$ & $\begin{array}{l}\text { December 2014- } \\
\text { July } 2017\end{array}$ & $\begin{array}{l}\text { Optionally } \\
\text { open access }\end{array}$ \\
\hline 4 & $\begin{array}{l}\text { April 2014- } \\
\text { February } \\
2017\end{array}$ & $\begin{array}{l}\text { July 2017-March } \\
2019\end{array}$ & $\begin{array}{l}\text { Optionally } \\
\text { open access }\end{array}$ \\
\hline 5 & $\begin{array}{l}\text { February } \\
\text { 2017-March } \\
2019\end{array}$ & March 2019- & Open access \\
\hline 6 & March 2019- & & Open access \\
\hline
\end{tabular}

contribution in 2007, 2013 and 2019. When the manuscript was submitted, data contributions responding to the call in 2019 were not yet fully integrated into the TRY database. Therefore all analyses presented in this paper are based on versions 1-5 of the TRY database (Table 1). TRY version 5, released on 26 March 2019, contains 387 data sets providing 11.8 million trait records, accompanied by 35 million ancillary data, for 2,091 traits and 280,000 plant taxa, mostly at the species level (Table 2). Data coverage is still driven by a few large (often integrated) databases, but increasingly small data sets (mostly primary data) contribute to the overall coverage (Figure 3a). Plant trait data in TRY can be traced to $>10,000$ original references. This highlights the breadth of data integrated in the TRY database and its nature as database of databases, a 'second generation of data pooling' (M. Westoby, personal communication, August 24, 2009).

We now observe a tendency that new trait-based research is increasingly planned against the background of the TRY database. Coverage and availability of trait data in TRY stimulate trait-based research, which then often leads to the identification of unexpected data gaps. This motivates data mobilization and/or new measurements, which improve the availability of plant trait data for the research community, and-if contributed to TRY-help the database grow. Examples for such a 'feed-forward data integration loop' are provided in Box 1.

To support this process, in this article, we take stock of the data compiled in the TRY database and present emerging patterns of data coverage and representativeness with a focus on the identification of principal and systematic gaps. Finally, we discuss ways forward and the potential future role of the TRY initiative for the research community.

\section{2 | MATERIALS AND METHODS}

\section{1 | Plant trait data in the TRY database}

Plant traits can be classified as categorical (qualitative and ordinal) or quantitative (continuous) traits (Kattge et al., 2011). Some traits are rather stable within species (mostly categorical traits), and some of these can be systematically compiled from species checklists and floras (e.g. Weigelt, König, \& Kreft, 2019).

TAB LE 2 Data coverage from TRY version 1 to 5

\begin{tabular}{|c|c|c|c|c|c|c|c|c|c|}
\hline Version & Trait records & Entities & $\begin{array}{l}\text { Trait } \\
\text { records } \\
\text { per } \\
\text { entity }\end{array}$ & Traits & $\begin{array}{l}\text { Average } \\
\text { number of } \\
\text { records } \\
\text { per trait }\end{array}$ & Species & $\begin{array}{l}\text { Geo- } \\
\text { referenced } \\
\text { trait records }\end{array}$ & Sites & $\begin{array}{l}\text { Ancillary } \\
\text { data }\end{array}$ \\
\hline 1 & $2,077,640$ & $1,110,303$ & 1.87 & 661 & 3,143 & 57,591 & 682,108 & 8,276 & $4,439,783$ \\
\hline 2 & $2,376,231$ & $1,207,669$ & 1.97 & 743 & 3,198 & 65,746 & 871,582 & 8,513 & $4,758,033$ \\
\hline 3 & $5,783,482$ & $2,246,967$ & 2.57 & 1,149 & 5,033 & 92,146 & $2,201,242$ & 11,844 & $11,834,960$ \\
\hline 4 & $7,162,252$ & $3,435,238$ & 2.08 & 1,981 & 3,615 & 141,461 & 2,978,776 & 16,480 & $14,644,354$ \\
\hline 5 & $11,850,781$ & $5,102,993$ & 2.37 & 2,091 & 5,668 & 279,875 & $4,952,839$ & 20,953 & $35,516,190$ \\
\hline
\end{tabular}



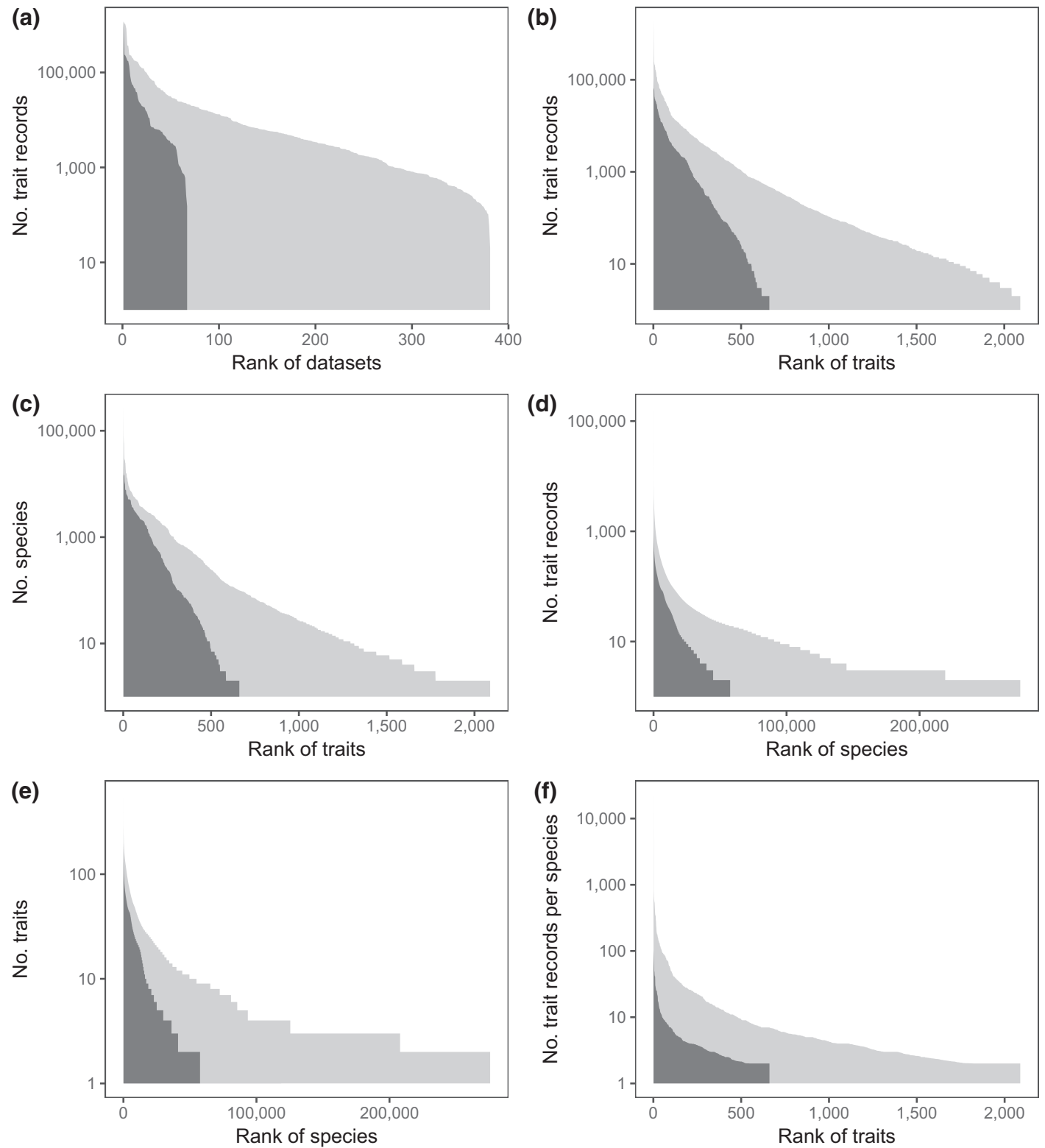

FIGURE 3 Trait data coverage of TRY version 1 (dark grey) and 5 (light grey). Data coverage in TRY is characterized by long-tailed ranksize distributions: (a) rank of dataset by trait records, (b) rank of traits by number of records, (c) rank of traits by number of species, (d) rank of species by trait records, (e) rank of species by number of traits, (f) rank of traits by number of records per species (averaged by trait). Note that $y$-axes are log-scaled

However, most traits relevant to ecology and earth system sciences are characterized by intraspecific variability and trait-environment relationships (mostly quantitative traits). Both kinds of traits are compiled in the TRY database, but with a focus on continuous traits. These traits have to be measured on individual plants in their particular environmental context. Each such trait measurement has high information content as it captures the specific response of a given genome to the prevailing environmental conditions. The collection of these quantitative traits and their essential environmental covariates is important but often tedious and expensive: researchers need to travel to the objects of interest-often to remote places-or they need to develop experiments creating specific environmental conditions. While trait measurements themselves may be relatively simple, the selection of the adequate entity (e.g., a representative plant in a community, or a representative leaf on a tree) and obtaining the relevant ancillary data (taxonomic identification, soil and climate properties, disturbance history, etc.) may require sophisticated instruments and a high degree of expertise and experience. Besides, these data are most often individual measurements with a low level of automation. This not only limits the number of measurements but also causes a high risk of errors, which need to be corrected a posteriori, requiring substantial human work. The 


\section{BOX 1 Examples for the 'feed-forward data integration loop' observed in the context of the TRY database}

- Iversen et al. (2017) indicated that in the TRY database only 1\% of trait records were related to roots. This motivated the development of the Fine-Root Ecology Database (FRED) specializing in the mobilization of fine-root trait records from the literature (Iversen et al., 2017). In the meantime, the first versions of the FRED database have been contributed to the TRY database. The improved number and availability of trait data on roots allowed for a project on root trait functionality in a whole-plant context (https://www.idiv.de/en/sdiv/working_groups/wg_pool/sroot.html), which motivated additional mobilization of root trait data.

- The promising coverage of plant trait data from tundra regions in the TRY database encouraged the inclusion of plant traits in an analysis of tundra ecosystem change, scaling shrub expansion from site to biome (Bjorkman, Myers-Smith, Elmendorf, Normand, Rüger, et al., 2018). In the context of the project, a large number of additional trait data were mobilized by the Tundra Traits Team (Bjorkman, Myers-Smith, Elmendorf, Normand, Thomas, et al., 2018), which have recently been contributed to the TRY database.

- Moreno-Martínez et al. (2018) estimated the worldwide variation of several leaf traits to improve the parameterization of global vegetation models and remote sensing approaches predicting, for example, gross primary productivity. Due to the low representation of traits for crop species in the TRY database, they could not provide estimates for major agricultural regions (see white spots in figure 5 of Moreno-Martínez et al., 2018). The identification of these gaps motivated mobilization of trait data for crop plants and agro-ecosystems (Engemann et al., 2016; Martin, Hale, et al., 2018; Martin \& Isaac, 2015), which were then contributed to the TRY database.

- Trait data on plant growth form (tree, shrub, herb, etc.) were compiled by TRY, extended and consolidated in the context of the BIEN initiative (Engemann et al., 2016; Enquist, Condit, Peet, Schildhauer, \& Thiers, 2016) and then contributed to the development of the GIFT database (Weigelt, König, \& Kreft, 2019). The upgraded plant growth form data were contributed again to TRY.

- Plant species richness is unequally distributed across the globe, with the highest species richness observed in the tropics (von Humboldt, 1817). The highest numbers of species with measurements in TRY are also found in the tropics, but as well the largest gap relative to reported species richness: less than $1 \%$ of estimated species richness is represented in TRY (Jetz et al., 2016). This principal and systematic mismatch of data coverage and representativeness has contributed to motivate the development of a 'global biodiversity observatory' of in situ measurements and space-borne remote sensing that tracks temporal changes in plant functional traits around the globe to fill critical knowledge gaps, aid in the assessment of global environmental change and improve predictions of future change (Jetz et al., 2016).

- TRY is involved in the sPlot initiative to establish a global vegetation-plot database (www.idiv.de/en/splot.html). sPlot supports the analysis of plant communities across the world's biomes by combining vegetation-plot data with traits from the TRY database (Bruelheide et al., 2019). This has resulted, for the first time, in global analyses of plant functional community data (Bruelheide et al., 2018). In contrast to single species measurements or trait values aggregated in grid cells, using vegetation-plot data allows understanding the role of traits for biotic interactions and community assembly processes. In turn, trait data measured in the context of sPlot are contributed to TRY.

integration of these data from different sources into a consistent data set requires a carefully designed workflow with sufficient data quality assurance (see Box 2: TRY data integration workflow).

These measurements of quantitative traits are single sampling events for particular individuals at certain locations and times, which preserve relevant information on intraspecific variation and provide the necessary detail to address questions at the level of populations or communities. Within individual field campaigns or experiments, researchers often aim to measure complete sets of these data: all traits of interest for all individuals or species in the analyses. However, across studies and data sets and at large scales, the coverage of these data shows major gaps, which provide major challenges concerning data completeness and representativeness (König et al., 2019).

\section{3 | RESULTS}

\section{1 | Data coverage}

Compared to TRY database version 1 and the state reported in Kattge et al. (2011), TRY version 5 has substantially grown with respect to the number of trait records, traits, species, entities, georeferenced measurement sites and ancillary data (Table 2).

\section{2 | Trait records and entities}

The numbers of trait records (individual trait measurements) and entities (individual plants or plant organs on which the measurements have been taken) increased by a factor of about 6 for trait 


\section{BOX 2 The data integration workflow for the TRY database version 5}

\section{Data acquisition}

In the context of the TRY initiative, data acquisition so far relies on active contributions by the community-data sets need to be sent by email or uploaded at the TRY website (https://www.try-db.org/TryWeb/Submission.php). From time to time (2007, 2013 and 2019), TRY sends out calls for data contributions to the community. However, so far there has been no systematic screening of public data repositories like DRYAD or PANGAEA for plant trait data.

\section{Data integration}

The basic principle of data integration in the TRY database is to preserve the original trait and ancillary data and annotate these with complementing and consolidated information. Data integration consists of three major components: data consolidation, complementation and quality assurance. We here provide a brief overview; a detailed description can be found in Supporting Information and on the TRY website (https://www.try-db.org/TryWeb/Database.php).

\section{Data consolidation}

The data structure is transformed into the entity-attribute-value (EAV) model and the OBOE schema (Madin et al., 2007) as used in the TRY database: a long table of trait records and ancillary data where all trait records and ancillary data measured on the same entity (individual plant or plant organ on which the measurements have been taken) are linked by a unique identifier. Plant taxonomy is consolidated using the Taxonomic Names Resolution Service (TNRS; http://tnrs.iplantcollaborative.org; Boyle et al., 2013) with a taxonomic backbone based on the Plant List (http://www.theplantlist.org), Missouri Botanical Garden's Tropicos database (http:// www.tropicos.org), the Global Compositae Checklist (https://www.compositae.org/checklist), the International Legume Database and Information Service (http://www.ildis.org), and USDA's Plants Database (http://plants.usda.gov). Trait names and definitions are consolidated across all data sets, based on the TOP thesaurus of plant characteristics (Garnier et al., 2017) or the plant trait handbook (Pérez-Harguindeguy et al., 2013), if possible. For continuous traits with more than 1,000 records, units are standardized and trait values recalculated. Most relevant ancillary data-geo-references, measurement date, exposition, maturity, and health-are consolidated across data sets and, if possible, to external standards, like the decimal representation of latitude and longitude, or ISO 8601 (YYYY-MM-DD) for the date.

\section{Data complementation}

After consolidation, additional trait values are derived from contributed trait data where possible; for example, leaf nitrogen content per area from leaf nitrogen content per dry mass and specific leaf area (SLA) if both were measured on the same entity.

Data quality assurance

Continuous traits with $>1,000$ records in the database are subject to a three-step process: (a) Systematic errors, like a wrong unit for a given trait for all records of a specific data set, are identified across data sets with semi-automated procedures and corrected. (b) Z-scores are calculated for each standardized trait value to indicate outliers and potential errors of individual trait records. (c) Duplicate trait records are identified based on consolidated trait names, taxonomy, units and values. Geo-references are checked against the ESA CCI Land Cover Map of Global Water Bodies (https://www.esa-landcover-cci.org/?q=node/162) to assure at least that the provided locations are on land.

After a data set has been integrated into the TRY database, the data set custodian is asked for feedback; that is, whether consolidated trait names are appropriate and consolidated values correct. Data are reformatted for data release and format errors (i.e. tabs and line breaks in database cells) are corrected. Finally, the original and consolidated data (including flags for outliers and duplicates) are released on request as tab-delimited text files.

records and 5 for entities from TRY version 1 (2.1 million trait records measured on 1.1 million entities) to TRY version 5 (11.8 million trait records measured on 5.0 million entities). The average number of trait records per entity increased from 1.9 to 2.4 (Table 2).

\section{3 | Traits}

The number of traits has grown steadily from TRY version 1 to 5 , apart from a steep step from TRY version 3 to 4 (Table 2). This step was caused by the contribution of the FRED database, which added about 700 new traits for roots. Data coverage across traits is characterized by long-tail distributions: a small number of traits is well covered by records and species, while the majority of traits has only very low coverage of records and species (Figure 3). However, the number of continuous traits with more than 1,000 records (which are subject to intense data quality assurance during integration) has increased from about 200 in TRY version 1 to 600 in TRY version 5 (Figure 3b). The number of traits with data for more than 100 species has increased from 300 to 700 (Figure 3c). In parallel, the number of records per trait and species ('intraspecific retakes') has increased from almost zero traits with on average more than 10 records per 
trait-species combination in TRY version 1 to more than 500 in TRY version 5 (Figure 3f).

The traits with the best species coverage in TRY version 5 are mostly categorical (Table 3). The categorical traits used for the

TABLE 3 Traits with best species coverage. The 30 traits covering the highest number of species in the TRY database version 5 and the number of species represented for these traits in TRY version 1. Data type: cat $=$ categorical; con $=$ continuous. Sorted by the number of species in TRY 5

\begin{tabular}{|c|c|c|c|}
\hline \multirow[b]{2}{*}{ Trait name } & \multirow{2}{*}{$\begin{array}{l}\text { Data } \\
\text { type }\end{array}$} & \multicolumn{2}{|c|}{ Number of species } \\
\hline & & TRY 1 & TRY 5 \\
\hline Plant growth form & cat & 31,327 & 263,357 \\
\hline Plant woodiness & cat & 14,628 & 79,298 \\
\hline Leaf type & cat & 7,934 & 62,904 \\
\hline Leaf compoundness & cat & 7,998 & 57,922 \\
\hline Leaf photosynthesis pathway & cat & 15,609 & 37,315 \\
\hline Leaflet number per leaf & con & 0 & 30,296 \\
\hline Plant height vegetative & con & 13,899 & 28,944 \\
\hline Leaf phenology type & cat & 14,622 & 28,514 \\
\hline Species tolerance to frost & cat & 2,180 & 28,122 \\
\hline Seed dry mass & con & 14,602 & 27,022 \\
\hline $\begin{array}{l}\text { Species occurrence range: } \\
\text { native versus invasive }\end{array}$ & cat & 11,313 & 25,067 \\
\hline Plant lifespan & $\begin{array}{l}\text { cat, } \\
\text { con }\end{array}$ & 7,617 & 24,712 \\
\hline Dispersal syndrome & cat & 7,528 & 21,717 \\
\hline $\begin{array}{l}\text { Plant nitrogen fixation } \\
\text { capacity }\end{array}$ & cat & 10,504 & 18,247 \\
\hline Leaf area ${ }^{a}$ & con & 8,873 & 16,663 \\
\hline $\begin{array}{l}\text { Leaf area per leaf dry mass } \\
\text { (specific leaf area) }^{b}\end{array}$ & con & 7,879 & 16,460 \\
\hline Plant resprouting capacity & cat & 3,320 & 15,997 \\
\hline $\begin{array}{l}\text { Seed germination rate (germina- } \\
\text { tion efficiency) }\end{array}$ & con & 6,698 & 15,822 \\
\hline Plant life form sensu Raunkiaer & cat & 7,710 & 15,766 \\
\hline Pollination syndrome & cat & 4,064 & 15,631 \\
\hline Leaf shape & cat & 3,191 & 15,594 \\
\hline Flower sex & cat & 3,572 & 13,735 \\
\hline $\begin{array}{l}\text { Leaf distribution arrangement } \\
\text { type }\end{array}$ & cat & 3,998 & 13,130 \\
\hline $\begin{array}{l}\text { Leaf nitrogen content per leaf } \\
\text { dry mass }\end{array}$ & con & 6,291 & 12,238 \\
\hline Stem specific density & con & 9,813 & 11,001 \\
\hline Flower colour & cat & 4,747 & 10,507 \\
\hline Seed storage behaviour & cat & 10,161 & 10,161 \\
\hline Fruit type & cat & 3,644 & 9,573 \\
\hline Leaf margin type & cat & 0 & 9,179 \\
\hline Wood growth ring distinction & cat & 5,121 & 9,103 \\
\hline
\end{tabular}

an case of compound leaves: leaflet, undefined if petiole is included or excluded.

b Undefined if petiole is included or excluded. classification of PFTs-plant woodiness, plant growth form, leaf type (broadleaved vs. needle-leaved), leaf phenology type (deciduous vs. evergreen), leaf photosynthesis pathway (C3, C4, CAM)-are still among the best covered. However, the number of species characterized for each of these traits has substantially increased from TRY version 1 to 5, most significantly for plant growth form from 31,327 to 263,357 species, supported by the contribution from the GIFT database (Weigelt, König, \& Kreft, 2019).

The quantitative traits with the highest species coverage are still the six traits which were already prominent in TRY version 1 and involved in the analysis of the global spectrum of plant form and function (Díaz et al., 2016): plant height, seed mass, leaf area, leaf area per dry mass, leaf nitrogen content per dry mass and stem specific density (SSD). However, in general, the coverage of continuous traits already present in TRY 1 has substantially improved. This facilitates a more robust characterization of frequency distributions (Figure 4). In most cases, the range of observed trait values did not change much from TRY version 1 to 5 , but the shapes of frequency distributions became more regular and pronounced, especially for multimodal frequency distributions like plant height and leaf $\Delta 13 \mathrm{C}$. Noteworthy, the examples in Figure 4 lack several kinds of traits because they are missing relevant numbers of trait records, like roots (only one trait), flowers and dead plant material (litter), secondary metabolites or data related to trophic interactions.

The 30 traits that were most often requested (Table 4) are dominated by continuous traits related to the global spectrum of plant form and function (Díaz et al., 2016), the leaf economics spectrum (Wright et al., 2004) and rooting depth. Only seven categorical traits are among these 30 traits. This indicates a switch between well covered-categorical-traits (Table 3 ) and most frequently requested-continuous-traits (Table 4). The first five most documented traits are categorical whereas among the 10 most requested traits, only one is categorical. However, within continuous traits, there is, in general, a good match between traits characterized for most species and traits most often requested. To some extent this may be influenced by the amount of available data for the individual traits. However, a noteworthy exception is rooting depth, as $10 \%$ of requests ask for this trait, while it is 'only' covered for 3,886 species, mostly contributed via the Global Dataset of Maximum Rooting Depth (Fan, MiguezMacho, Jobbágy, Jackson, \& Otero-Casal, 2017). This mismatch indicates a demand for more data on the most relevant belowground traits.

\section{4 | Species}

From TRY version 1 to 4, the number of species increased slowly, but almost doubled to version 5 due to the contribution of plant growth form data from the GIFT database, which added about 100,000 new species. As in the case of traits, the data coverage for species is characterized by long-tail distributions: few species are covered well by measurements and traits, while the majority 

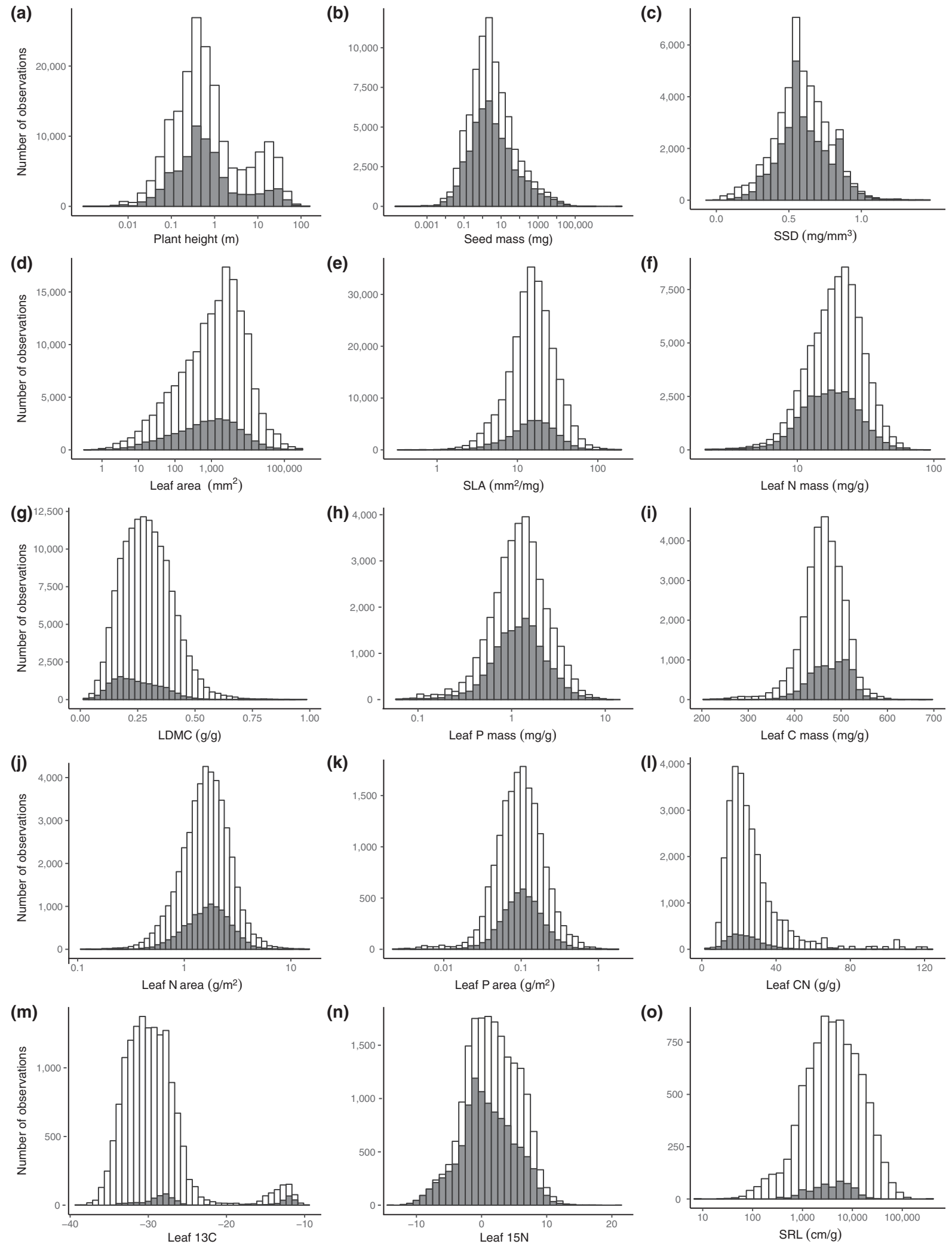

FIGURE 4 Frequency distributions of observations for 15 ecologically relevant and well sampled continuous traits from different plant organs. Grey: TRY version 1; white: TRY version 5. (a) Plant height, (b) Seed mass, (c) SSD: stem dry mass per stem fresh volume (stem specific density), (d) Leaf area, (e) SLA: leaf area per leaf dry mass (specific leaf area), (f) Leaf N mass: leaf nitrogen content per leaf dry mass (leaf nitrogen concentration), (g) LDMC: leaf dry mass per leaf fresh mass (leaf dry matter content), (h) Leaf P mass: leaf phosphorus content per leaf dry mass, (i) Leaf $C$ mass: leaf carbon content per leaf dry mass, (j) Leaf $N$ area: leaf nitrogen content per leaf area, (k) Leaf $P$ area: leaf phosphorus content per leaf area, (I) Leaf CN: leaf carbon content per leaf nitrogen content, (m) Leaf 13C: leaf 13C carbon isotope signature, leaf $\Delta 13 \mathrm{C}$, (n) Leaf $15 \mathrm{~N}$ : leaf $15 \mathrm{~N}$ nitrogen isotope signature, (o) SRL: root length per root dry mass (specific root length) 
TAB LE 4 Most often requested traits. The 30 traits with the highest number of requests (status 1 October 2019). Number of requests and in parentheses the percentage relative to all 7,330 requests

\begin{tabular}{|c|c|c|}
\hline Trait & Data type & $\begin{array}{l}\text { Number of } \\
\text { requests }\end{array}$ \\
\hline $\begin{array}{l}\text { Leaf area per leaf dry mass (specific } \\
\text { leaf area or } 1 / \text { LMA) }\end{array}$ & con & $2,977(41 \%)$ \\
\hline Plant height vegetative & con & $2,159(29 \%)$ \\
\hline $\begin{array}{l}\text { Leaf nitrogen }(\mathrm{N}) \text { content per leaf dry } \\
\text { mass }\end{array}$ & con & $1,938(26 \%)$ \\
\hline Leaf area $^{\mathrm{b}}$ & con & $1,676(23 \%)$ \\
\hline Plant growth form & cat & $1,625(22 \%)$ \\
\hline Seed dry mass & con & $1,580(22 \%)$ \\
\hline $\begin{array}{l}\text { Leaf nitrogen }(\mathrm{N}) \text { content per leaf } \\
\text { area }\end{array}$ & con & $1,221(17 \%)$ \\
\hline $\begin{array}{l}\text { Leaf phosphorus }(\mathrm{P}) \text { content per leaf } \\
\text { dry mass }\end{array}$ & con & $1,170(16 \%)$ \\
\hline Plant lifespan (longevity) & con & $1,168(16 \%)$ \\
\hline $\begin{array}{l}\text { Leaf dry mass per leaf fresh mass (leaf } \\
\text { dry matter content) }\end{array}$ & con & $1,147(16 \%)$ \\
\hline Leaf phenology type & cat & $1,047(14 \%)$ \\
\hline $\begin{array}{l}\text { Leaf carbon }(C) \text { content per leaf dry } \\
\text { mass }\end{array}$ & con & $973(13 \%)$ \\
\hline Dispersal syndrome & cat & $958(13 \%)$ \\
\hline Stem specific density & con & $951(13 \%)$ \\
\hline Leaf photosynthesis rate per leaf area & con & $896(12 \%)$ \\
\hline Leaf dry mass (single leaf) & con & $896(12 \%)$ \\
\hline Leaf photosynthesis pathway & cat & $874(12 \%)$ \\
\hline Leaf thickness & con & $852(12 \%)$ \\
\hline Plant nitrogen $(\mathrm{N})$ fixation capacity & con & $833(11 \%)$ \\
\hline Leaf carbon/nitrogen $(\mathrm{C} / \mathrm{N})$ ratio & con & $817(11 \%)$ \\
\hline Plant life form sensu Raunkiaer & cat & 801 (11\%) \\
\hline Leaf lifespan (longevity) & con & $790(11 \%)$ \\
\hline Root rooting depth & con & $733(10 \%)$ \\
\hline Plant growth rate & con & $727(10 \%)$ \\
\hline Leaf type & cat & $727(10 \%)$ \\
\hline $\begin{array}{l}\text { Leaf phosphorus }(\mathrm{P}) \text { content per leaf } \\
\text { area }\end{array}$ & con & 719 (10\%) \\
\hline Plant functional type & cat & 710 (10\%) \\
\hline Plant woodiness & cat & $708(10 \%)$ \\
\hline $\begin{array}{l}\text { Leaf photosynthesis rate per leaf dry } \\
\text { mass }\end{array}$ & con & $701(10 \%)$ \\
\hline Plant reproductive phenology timing & con & $672(9 \%)$ \\
\hline
\end{tabular}

${ }^{a}$ Undefined if petiole is included or excluded.

${ }^{b}$ In case of compound leaves: leaflet, undefined if petiole is included or excluded.

of species has only very low data coverage (Figure $3 d-f)$. The species characterized by the most traits tend to be northern temperate trees or globally distributed pasture species (Table 5). Out of the top 30 species with the best trait coverage, 27 (90\%) originate in Central or Northern Europe.
TABLE 5 Species with best trait coverage. The 30 species with the highest number of traits in the TRY database version 5 and number of traits represented for these species in TRY version 1 and 5 . Sorted by the number of traits in TRY 5

\begin{tabular}{|c|c|c|c|}
\hline \multirow[b]{2}{*}{ Species } & \multirow{2}{*}{$\begin{array}{l}\text { Plant } \\
\text { growth } \\
\text { form }\end{array}$} & \multicolumn{2}{|c|}{ Number of traits } \\
\hline & & TRY 1 & TRY 5 \\
\hline Pinus sylvestris & Tree & 264 & 569 \\
\hline Fagus sylvatica & Tree & 237 & 517 \\
\hline Picea abies & Tree & 252 & 475 \\
\hline Quercus robur & Tree & 194 & 435 \\
\hline Acer saccharum & Tree & 139 & 430 \\
\hline Betula pendula & Tree & 265 & 429 \\
\hline Achillea millefolium & Herb & 209 & 403 \\
\hline Acer pseudoplatanus & Tree & 186 & 397 \\
\hline Trifolium pratense & Herb & 181 & 395 \\
\hline Quercus rubra & Tree & 190 & 388 \\
\hline Dactylis glomerata & Herb & 193 & 387 \\
\hline Plantago lanceolata & Herb & 156 & 386 \\
\hline Vaccinium vitis-idaea & Shrub & 189 & 382 \\
\hline Trifolium repens & Herb & 173 & 380 \\
\hline Fraxinus excelsior & Tree & 196 & 378 \\
\hline Acer platanoides & Tree & 186 & 378 \\
\hline Quercus petraea & Tree & 194 & 368 \\
\hline Poa pratensis & Herb & 195 & 366 \\
\hline Holcus lanatus & Herb & 178 & 364 \\
\hline Tilia cordata & Tree & 153 & 362 \\
\hline Calluna vulgaris & Shrub & 190 & 360 \\
\hline Lotus corniculatus & Herb & 153 & 360 \\
\hline Pseudotsuga menziesii & Tree & 141 & 356 \\
\hline Medicago lupulina & Herb & 145 & 351 \\
\hline Festuca rubra & Herb & 175 & 347 \\
\hline Sorbus aucuparia & Tree & 197 & 335 \\
\hline Phleum pratense & Herb & 179 & 335 \\
\hline Quercus ilex & Tree & 195 & 333 \\
\hline Betula papyrifera & Tree & 126 & 332 \\
\hline Vaccinium uliginosum & Shrub & 169 & 330 \\
\hline
\end{tabular}

\subsection{Entity $\times$ trait and species $\times$ trait matrices}

The trait data in the TRY database can be represented by two two-dimensional matrices: the entity $\times$ trait matrix, with entities in rows and traits in columns; and the species $x$ trait matrix, with species in rows and traits in columns. Both matrices are characterized as large but sparse: high numbers of entities, species and traits in TRY make the two matrices large, but many cells in the matrices are empty. From TRY version 1 to 5 the size of the matrices has grown by a factor of 15 , but at the same time the number of trait records to fill 
the cells increased only by a factor of about 5 and thus the matrices became even sparser: the fractional coverage decreased from $0.4 \%$ to $0.1 \%$ (entity $\times$ trait) and $1.4 \%$ to $0.4 \%$ (species $\times$ trait; Figure $5 a$ ). This sparsity together with the observed long-tail distributions has consequences, especially for multivariate analyses. Given that on average only two to three traits of the 2,091 traits in TRY version 5 are measured on an individual plant (entity), a multivariate analysis based on individuals is indeed practically impossible, as mentioned by Shan et al. (2012). Even after aggregation at the species level, the decline of the number of species with complete trait coverage when adding a new trait, for example for multivariate analysis, is surprisingly high (Figure 5b). Additionally, the final number of species represented in the analysis is determined by the trait with the lowest species coverage. Therefore multivariate analyses with more than about six traits are still very much limited by the number of species. The same applies when species have to be classified by several categorical traits, like for example in the context of PFTs.

\section{6 | Ancillary data}

The numbers of ancillary data, geo-referenced trait records and trait records with measurement date increased by a factor of almost 10 from TRY version 1 to TRY version 5 (Table 2). The ratio of ancillary data to trait records, therefore, increased from TRY version 1 to 5 from 2:1 to 3:1 and the fraction of geo-referenced trait records from about $33 \%$ to $42 \%$ (Table 2). The number of geo-referenced trait records with information on measurement date that could be standardized to year, month and day increased from 290,000 in TRY version 1 (15\% of all trait records) to 2.5 million records in TRY version 5 (20\%). The increasing ratio of ancillary data to trait records indicates growing awareness for the relevance of environmental conditions during plant growth and trait measurements. In this context, geo-references (and date) are crucial, as they allow trait records to be related to information on climate, soil or biome type from external sources.

The geographic coverage of trait measurements has substantially improved from TRY version 1 (8,276 measurement sites representing $1,2601^{\circ} \times 1^{\circ}$ grid-cells) to TRY version 5 (20,953 sites representing

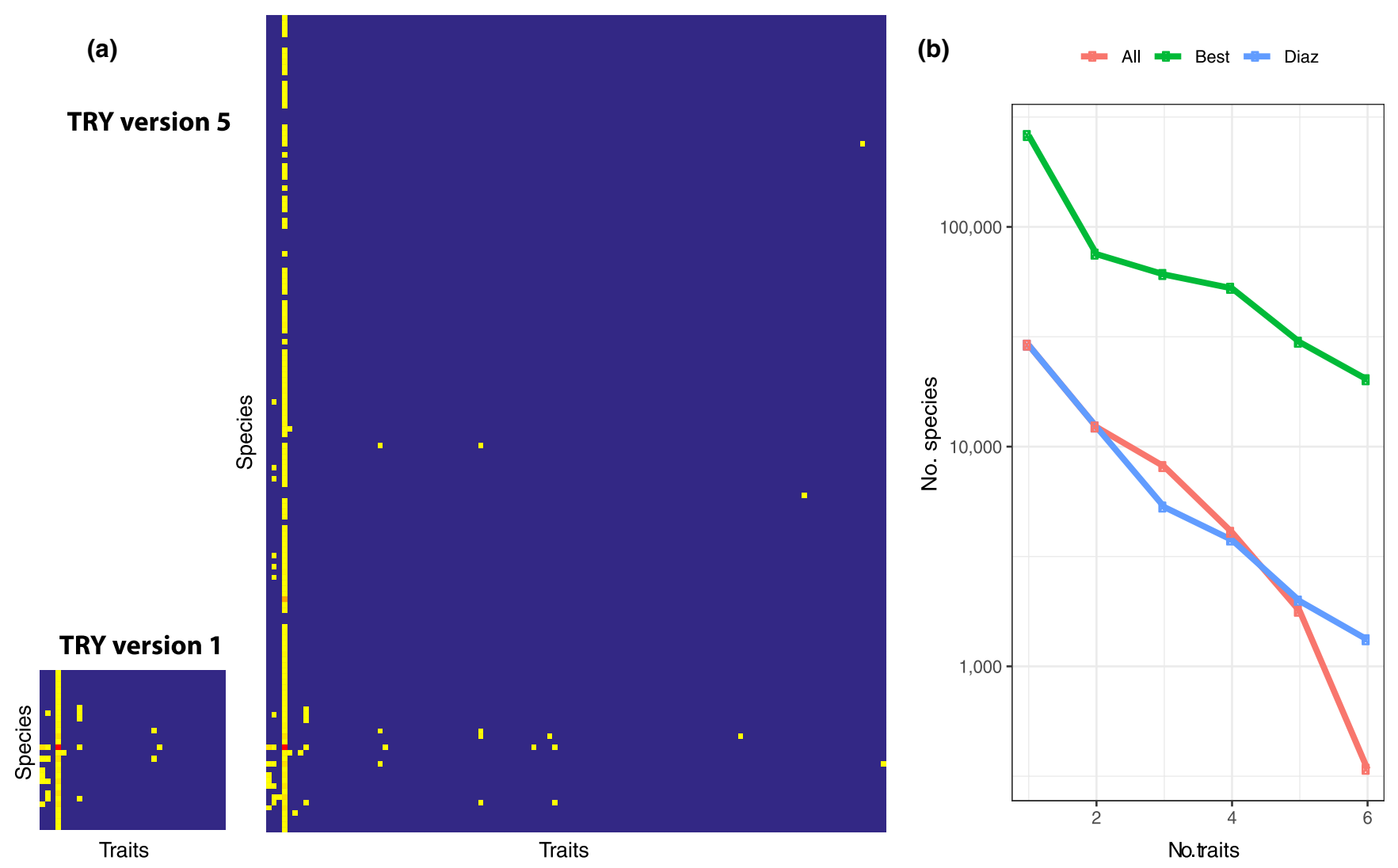

FIGURE 5 (a) Comparison of the species $\times$ trait matrix from TRY version 1 and version 5 . The sizes of the boxes represent the numbers of species and traits. Blue: missing data; bright colour: the presence of data (yellow to red indicating an increasing number of measurements). For visibility, only $5 \%$ of traits and $0.05 \%$ of species are shown (randomly selected, with TRY version 1 as a subsample of TRY version 5; ordering of species and traits by submission date). 'Plant growth form' with entries for almost all species has been selected coincidentally. (b) Multivariate analyses: the decline of the number of common species with an increasing number of traits. Green: the six best covered traits in TRY version 5 (categorical traits: plant growth form, plant woodiness, leaf type, leaf compoundness, leaf photosynthetic pathway, leaflet number per leaf); blue: six traits representing plant form and function in Díaz et al. (2016); red: the best covered quantitative traits representing each of the six plant parts (see Figure 6): shoot (plant height vegetative), reproductive organs (seed dry mass), whole plant (plant lifespan), leaves (SLA), roots (rooting depth) and dead material (litter decomposition rate) 


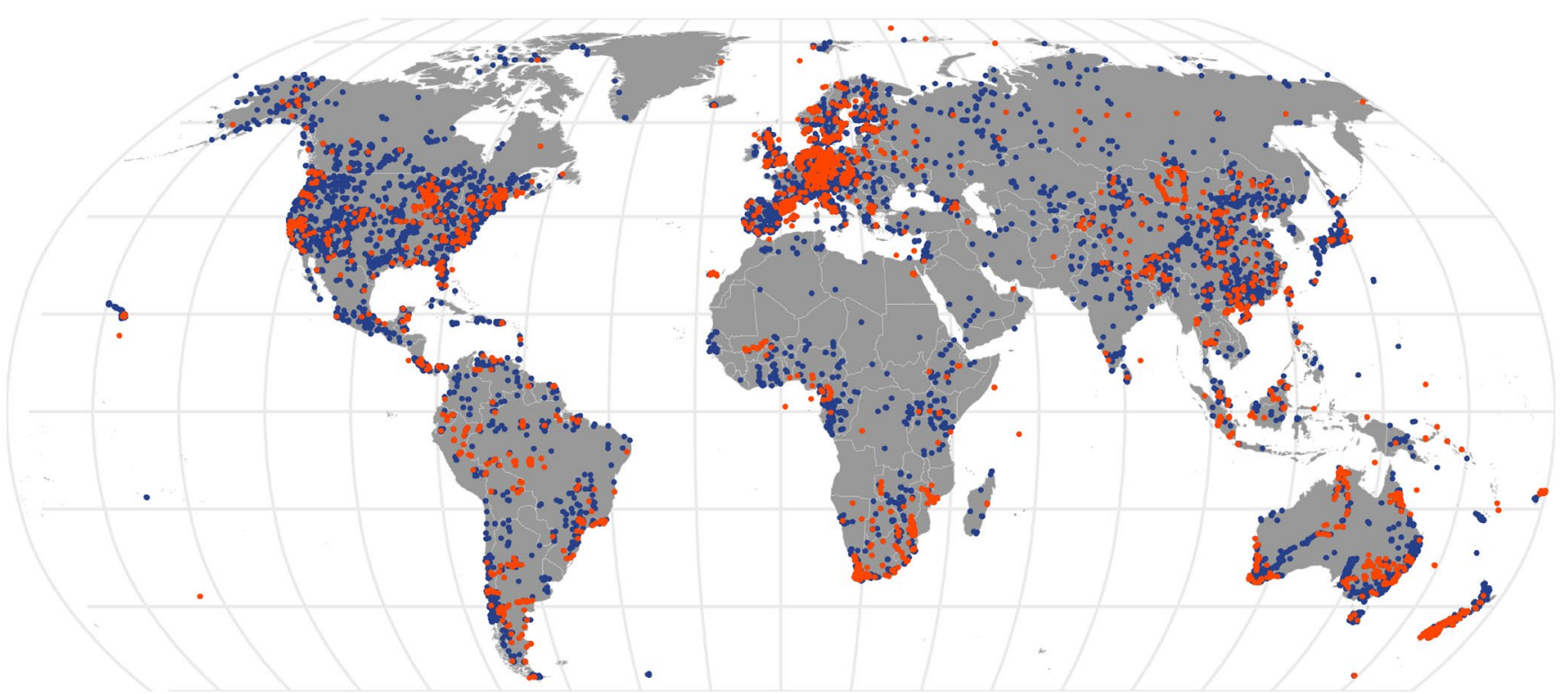

FIGURE 6 Geographic coverage of measurement sites in TRY version 1 (red) and additional measurement sites in TRY version 5 (blue)

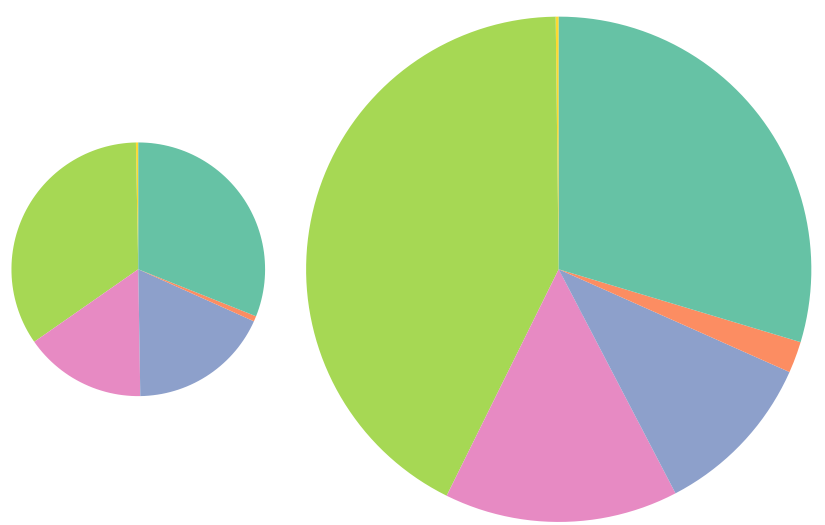

$3,3201^{\circ} \times 1^{\circ}$ grid-cells; Table 2; Figure 6). Europe still has the highest density of measurement sites, but TRY version 5 also provides good coverage for the United States and China. The number of measurement sites has substantially improved for several other regions as well, for example Central America, Russia, Asia and parts of central Africa. However, there are still obvious gaps in boreal regions (Canada, East Russia) and some parts of the tropics and subtropics, particularly in Africa (Figure 6).

\section{7 | Data completeness and representativeness}

To progress from a description of data coverage towards an analysis of representativeness, we need a baseline for comparison. At the global scale, this information has been lacking. Reference data sets have become available only recently for plant growth form (Weigelt, König, \& Kreft, 2019) and phylogeny (Smith \& Brown, 2018) representing about 260,000 and 356,000 of the 400,000 extant species. Together with estimations for the global distribution of plant species richness (Kier et al., 2005), it seems now possible and timely to explicitly address
FIGURE 7 Distribution of trait records among plant parts. Different sizes of circles indicate different data coverage in TRY version 1 (left, 2.1 million trait records) and TRY version 5 (right, 11.8 million trait records)

Root

Shoot

Reproductive organs

Whole plant

Dead material representativeness of plant trait data in the TRY database along five key dimensions: (a) Are trait data in TRY well distributed among plant parts? (b) Are the species in TRY and for individual traits representative for global plant growth forms and functional types? (c) Are the species in TRY and for individual traits representative according to phylogeny? (d) Does the geographic distribution of species richness in TRY represent the estimated pattern of global species richness? (e) Is data coverage sufficient to represent intraspecific variation?

\section{8 | Distribution of trait records among plant parts}

Trait records in the TRY database are very unequally distributed among different plant parts (Figure 7): leaves and the whole plant are well represented; shoot and reproductive organs are moderately represented; roots and dead material (morphological and chemical feature of litter and coarse woody debris, but also decomposition rates) are not well represented. Within reproductive organs, seeds are better represented than floral traits, mostly due to contributions from the Seed Information Database. The skewed distribution of 
trait records to the different parts of the plants has only changed little from TRY version 1 to 5 . However, the fraction of records for root traits has substantially increased (from $0.7 \%$ to $2.0 \%$ ), due to the contribution of the FRED database.

\section{9 | Plant growth form and PFTs}

The most basic approach to characterize functional groups of plant species and their impact on vegetation and ecosystem function is the plant growth form, with its simplest classification to herbs, shrubs and trees. We compare here the fraction of the different plant growth forms for trait measurements in TRY version 5 to a comprehensive baseline of plant growth form for $>280,000$ of the extant 400,000 species, which is currently developed in the context of the GIFT database project.

In GIFT, about $50 \%$ of species are currently assigned to herbs, $30 \%$ to trees and $20 \%$ to shrubs (Figure 8 ). This distribution is well reflected by the species in TRY (excluding data from the GIFT database). However, the six best covered continuous traits in TRY indicate that this distribution is very much trait dependent, with a bias towards trees versus herbs, while the fraction of shrubs is surprisingly constant and close to the fraction in the GIFT database (Figure 8). The overrepresentation of trees is most obvious for SSD, which is not surprising because SSD is a more general concept derived from wood density, a trait relevant for forestry, timber industry and estimates of forest vegetation biomass. However, the tendency of relatively more data for trees compared to other growth forms is also obvious for SLA, leaf nitrogen content per dry mass and leaf area, but opposite for root length per root dry mass (specific root length), which is frequently reported for herbs.

Apart from plant growth form, three additional categorical traits are relevant to determine PFTs commonly used in global vegetation models: leaf type (broadleaved vs. needle-leaved) and leaf phenology type (deciduous vs. evergreen) for tree species, and photosynthetic pathway (C3, C4, CAM) for herbaceous species. TRY provides leaf type and leaf phenology type, for about $10 \%$ of the estimated
130,000 tree species worldwide and photosynthetic pathway for about $6 \%$ of estimated 200,000 herb species.

\subsection{0 | Phylogeny}

Smith and Brown (2018) published a series of broadly inclusive seed plant phylogenies. Here we chose the most comprehensive phylogeny (ALLMB), containing 356,305 taxa, as a baseline to visualize the coverage of TRY in a phylogenetic context. Taxa in ALLMB were cut to binomials and consolidated using the TNRS with TPL, GCC, ILDIS, TROPICOS and USDA as the taxonomic backbone (the same approach as for TRY). After consolidation, we could match the taxa in the phylogeny to 208,406 of the 279,875 taxa in TRY. Higher level taxonomy is based on Zanne et al. (2014).

Visually, the 208,000 species with data in TRY are well distributed across the 350,000 species represented in the phylogeny of seed plants (Figure 9). An ancestral state reconstruction (ASR) of species trait number confirms that the long-tail distribution previously seen at the species level also holds in a phylogenetic context: some clades are covered very well (bright colours), while most clades have lower data coverage (dark colours). The ASR additionally shows how deep in phylogeny data gaps are rooted. This indicates the potential for, and limits to, phylogenetically or taxonometically informed gap-filling (Schrodt et al., 2015). Examples of high-coverage clades are (parts of) the Pinales, Poales and Asterales. When looking at the six best-covered continuous traits individually, we find these too are well distributed across the phylogeny (Figure 9).

\subsection{Geographic distribution of species richness}

Jetz et al. (2016) reported a latitudinal gradient in disparities between plant species with regional measurements in TRY and estimated species richness, with the largest gap observed in the tropics, because these are especially rich in species. To address this in more detail, we
FIGURE 8 Fraction of species with plant growth forms herb, shrub and tree in the GIFT database, in TRY version 5 (excl. GIFT) and for the six best covered continuous traits in TRY version 5: plant height, seed dry mass, leaf area per leaf dry mass (SLA), leaf $N$ per dry mass, leaf area and stem specific density (SSD), and one well covered root trait: specific root length (SRL). In parentheses: the number of species with data for the trait and the number of species for which the growth form could be determined as tree, shrub or herb

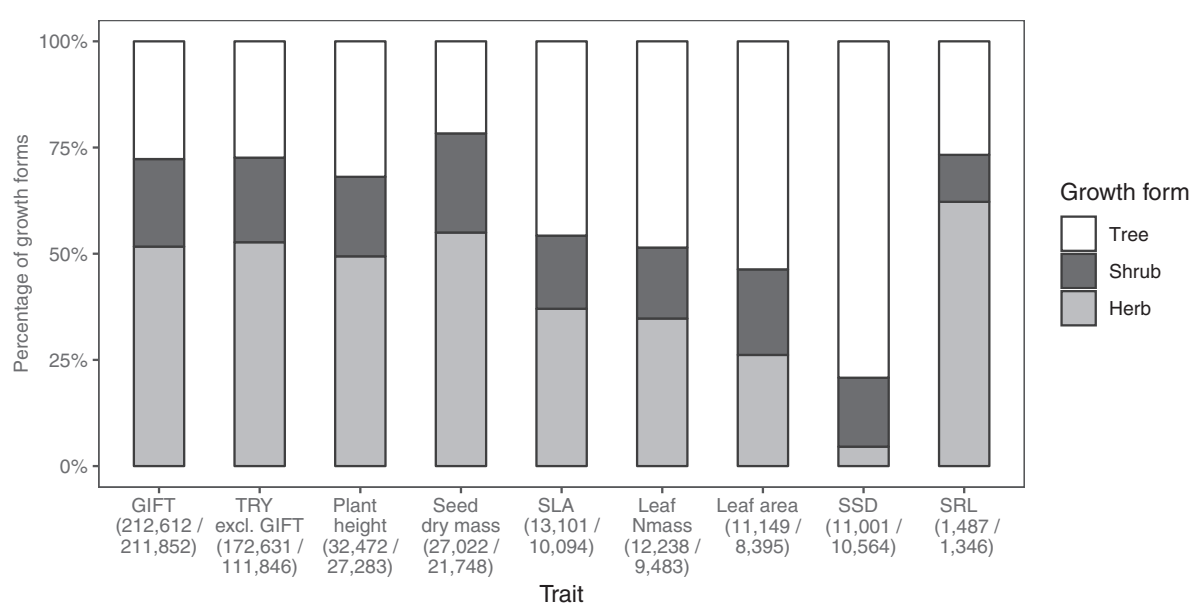




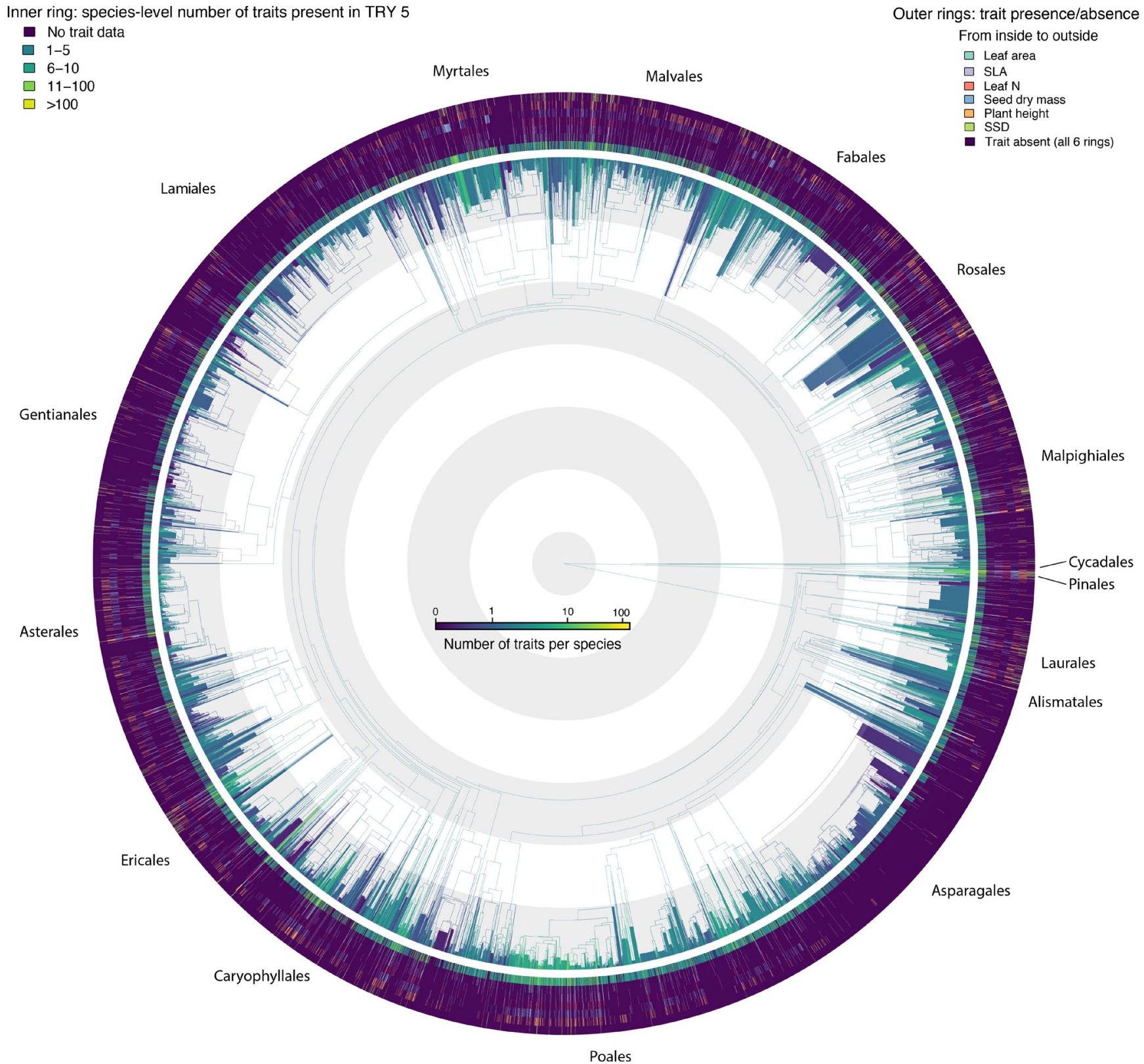

FIGURE 9 Trait coverage per species projected on a global phylogeny. The presence of trait data for plant species in the TRY database version 5 matched to the global ALLMB phylogeny published by Smith and Brown (2018). Rings surrounding the phylogeny indicate from inside outwards: (i) number of traits per species (innermost ring); (ii) presence of data for six of the best covered continuous traits, specifically: leaf area, leaf area per leaf dry mass (SLA), leaf nitrogen content per dry mass (LeafN), seed dry mass, plant height and stem specific density (SSD). Colours of phylogeny branches represent an ancestral state reconstruction of the number of traits per species. White and grey circles indicate periods of 50 million years. For visibility, only $5 \%$ of species (randomly selected) are presented

here ask if the TRY database provides trait information for a relevant number of plant species in the different regions worldwide. To characterize regions in an ecologically meaningful way we adopt the ecoregions introduced by Olson et al. (2001), which are defined as relatively large units of land containing a distinct assemblage of natural communities and species, with boundaries that approximate the original extent of natural communities before major land-use change. The ecoregions are nested within biomes with biotic communities formed in response to a shared physical climate, most importantly temperature and rainfall. We compare the number of species, which have trait measurements in an ecoregion in TRY version 5 to species numbers per ecoregion estimated by Kier et al. (2005). This approach accounts to some extent for intraspecific trait variation, as it counts only species with at least one trait measurement in the given ecoregion.

The 839 ecoregions defined by Olson et al. (2001) are very different in size, from $6 \mathrm{~km}^{2}$ (San Felix-San Ambrosio Islands temperate forests) to $4,639,920 \mathrm{~km}^{2}$ (Sahara desert) with species numbers ranging from 0 (St. Peter and St. Paul rocks and the Maudlandia Antarctic desert) to 10,000 (Borneo lowland rain forests). The TRY database contains no trait measurements for 271 mostly small ecoregions and 
up to 1,400 species for some ecoregions in Europe (Alps conifer and mixed forests) and tropical South America (Napo moist forests, Tapajos-Xingu moist forests). In general, high absolute numbers of species with trait measurements for ecoregions are found in Europe,
East Asia, Oceania, Australia, tropical South America and the United States (Figure 10a). East Asia, Oceania and tropical South America are also the regions with the highest numbers of species per ecoregions estimated by Kier et al. (2005; Figure $10 \mathrm{~b})$.

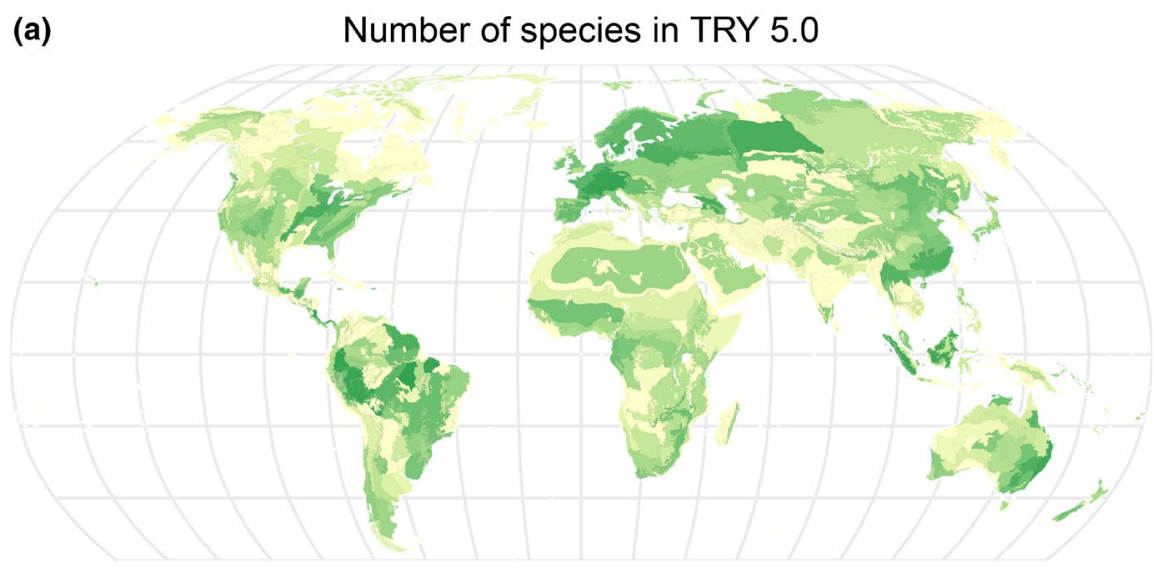

(b)

Number of species in Kier et al. (2005)

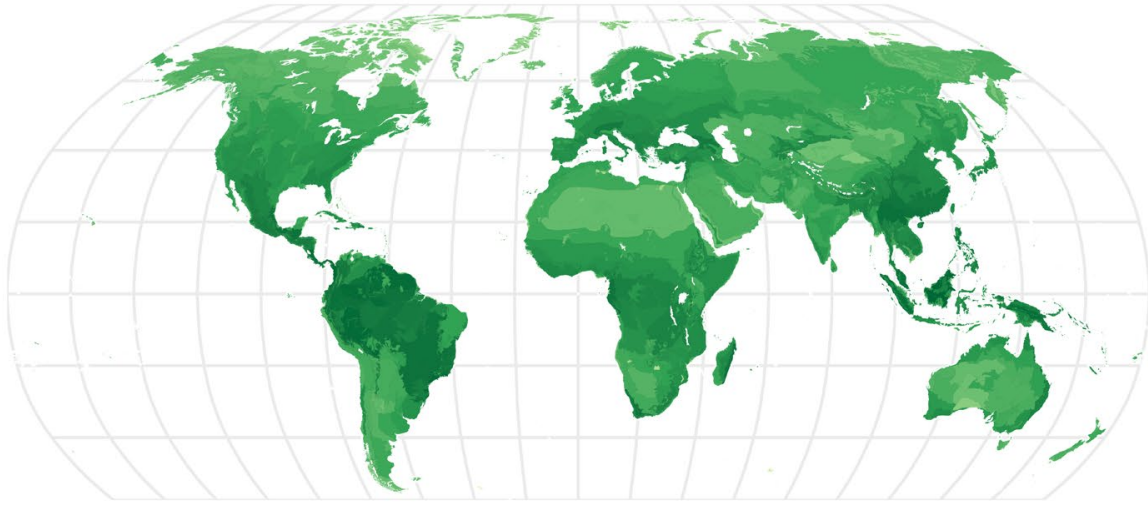

(c)

Proportion of species covered by TRY 5.0

FIGURE 10 Geographic representativeness: (a) the number of species with at least one trait measurement in an ecoregion in TRY version 5; (b) number of species per ecoregion estimated by Kier et al. (2005); (c) fraction of species represented in TRY version 5 versus number of species per ecoregion estimated by Kier et al. (2005)

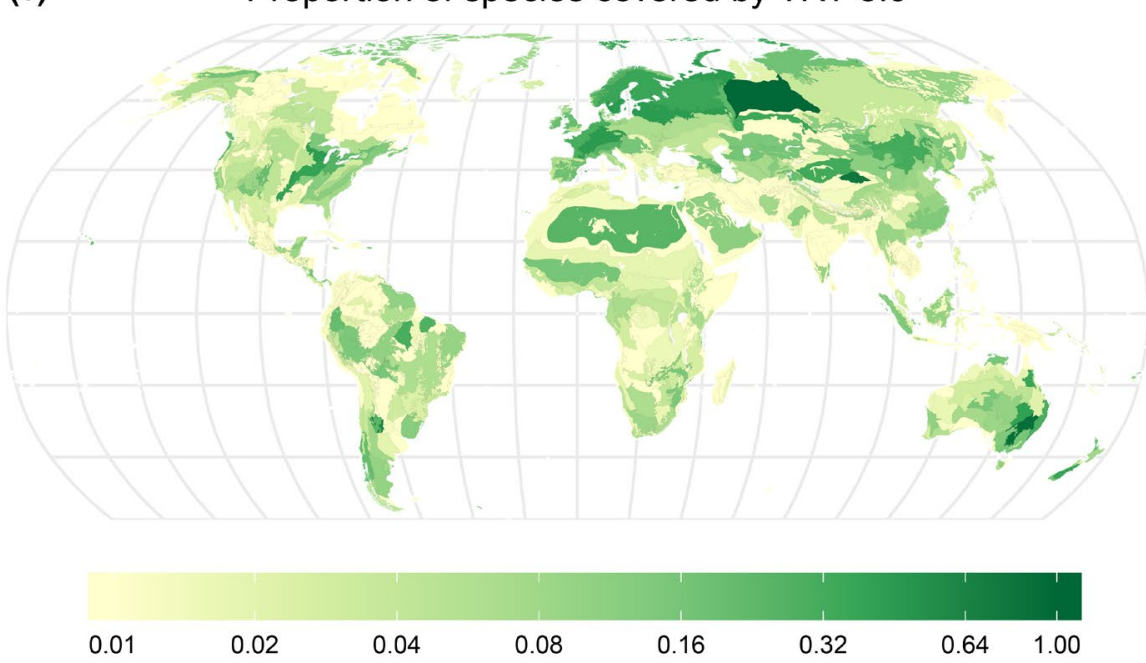




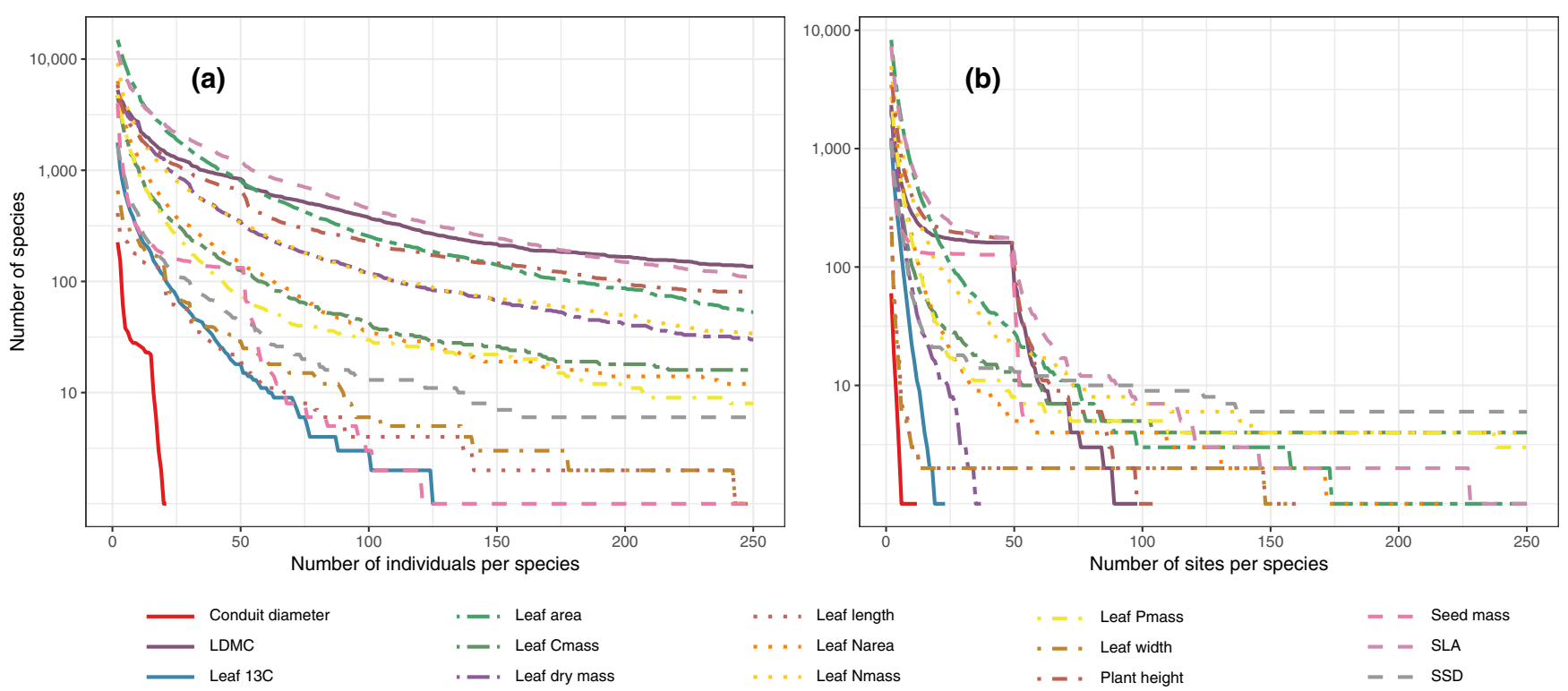

FIGURE 11 Data coverage enabling examination of intraspecific variation: impact of the minimum number of individuals per species (a) and measurement sites per species (b) on the number of species available for analyses of intraspecific variation. Measurement sites were classified as different if they differed at least by 0.01 degrees in latitude or longitude. LDMC: leaf dry matter content; Leaf 13C: Leaf carbon (C) isotope signature (leaf $\Delta 13 C$ ); Leaf $C$ mass: leaf carbon content per leaf dry mass; Leaf $N$ area: leaf nitrogen content per leaf area; Leaf $\mathrm{N}$ mass: leaf nitrogen content per leaf dry mass; Leaf P mass: leaf phosphorus content per leaf dry mass; SLA: leaf area per leaf dry mass (specific leaf area); SSD: stem specific density

The best relative coverage in TRY (Figure 10c) is provided for the Marielandia Antarctic tundra (two species estimated and in TRY) and for a large ecoregion in Central Russia (West Siberian taiga, 900 species estimated, 885 in TRY). The species in the Russian ecoregion are measured at several sites relatively well distributed across the ecoregion, but dominated by just one trait, 'mycorrhiza infection intensity' contributed by the mycorrhizal intensity database (Akhmetzhanova et al., 2012). Some other ecoregions are also well covered with data for more than $50 \%$ of estimated species (Southeast Australia temperate savanna, Qaidam Basin semi-desert, Córdoba forests and mountain grasslands). Apart from these individual ecoregions spread across the world, large parts of Europe are well covered, with trait data for about $30 \%$ of the species number estimated by Kier et al. (2005). Some ecoregions in East Asia, Australia, tropical South America, the Sahara, and the United States are also well covered, providing data for about $20 \%$ of estimated species richness. Very low relative coverage $(<2 \%)$ is observed for major parts of Canada, Africa, western Asia (Iran, Iraq, Pakistan, Afghanistan) and major parts of India.

\subsection{2 | Intraspecific variation}

Understanding and predicting intraspecific variation for a relevant number of traits and species is still in its infancy. Given that TRY is collecting trait measurements on individual plants, the TRY data set might be suited to address these questions. A precondition for such analyses is a minimum number of measurements on different individual plants at different sites per trait and species. To assess this issue, we plotted the number of species for which TRY version 5 contains a minimum number of individual measurements (Figure 11a) and the number of species for which TRY version 5 contains measurements from a minimum number of individual sites (Figure 11b) for the 15 best-covered continuous traits. By increasing the minimum number of individuals or sites, the number of species available for analysis decreases sharply (more than exponentially), whereas the exact slope is trait specific. The characterization of intraspecific variation in Kattge et al. (2011) relied on at least 20 individuals per species. Based on this criterion, TRY 5 provides information for hundreds to thousands of species for 14 out of the 15 traits (Figure 11a). Assuming a more realistic limit of 100 individuals per species, SLA and LDMC are sufficiently covered for about 300 species, and four other traits for more than 100 species. Assuming a minimum number of 200 individuals per species, four traits still allow for an analysis of 100 species, and nine traits allow for the analysis of intraspecific variation of more than 10 species. However, the numbers are more humbling if the environmental context is taken into account (Figure 11b). If we assume that trait records from a minimum number of 50 sites per species are necessary to represent intraspecific variation, four traits are sufficiently covered for about 100 species. If 100 sites are necessary, no trait is covered by data for more than 10 species.

\section{4 | DISCUSSION}

Plant trait data provide a wealth of information directly relevant in several scientific contexts, from conservation, ecology and evolution to earth system sciences. To fully realize this potential, the TRY 
initiative was initiated in 2007 as a 'database of databases' and leading groups in the field of functional plant ecology joined forces for this community-driven program. The TRY database now provides an unprecedented number of consolidated plant trait data, which have become easily accessible at the TRY Data Portal under an open access data policy.

The TRY database is well accepted by the scientific community and has facilitated progress in different aspects of research, for example in global vegetation modelling from static PFTs to a more continuous representation of biodiversity (e.g. Peaucelle, Bellassen, Ciais, Peñuelas, \& Viovy, 2016; Sakschewski et al., 2015, 2016; Verheijen et al., 2013, 2015), extending macroecology and biodiversity by functional aspects (e.g. Bjorkman, Myers-Smith, Elmendorf, Normand, Rüger, et al., 2018; Bruelheide et al., 2018; Craven et al., 2018; Newbold et al., 2015), linking soil characteristics to vegetation attributes (e.g. Boeddinghaus et al., 2019; de Vries et al., 2012; Delgado-Baquerizo et al., 2018) and providing data for global maps of plant traits (e.g. Butler et al., 2017; Moreno-Martínez et al., 2018). The central keywords of the cluster analysis in Figure 2 (biodiversity, climate change, plant traits, functional diversity, carbon cycle, community, vegetation and environmental filtering) seem to reflect the expectation that improved knowledge of plant functional diversity, mediated by plant traits, contributes to a better understanding of vegetation feedbacks to climate change and drivers and consequences of plant biodiversity loss.

Data coverage of the TRY database is characterized by four attributes: (a) long-tail distributions, (b) sparse matrices, (c) increasing number of ancillary data per trait record and (d) increasing geographic coverage. So far the size of the two sparse matrices (entity $\times$ trait and species $\times$ trait) has increased faster than the number of trait records to fill the matrices. Therefore the sparseness of the matrices has increased from TRY version 1 to 5 (the fractional coverage declined). Rather than converging in a small number of traits, the scientific community continues to measure a large, diverse number of traits, following equally diverse motivations.

However, given the number of species has a natural limit and assuming the number of traits will continue to grow, but more slowly, once the most obvious ones have been covered, we expect that the sparseness of the entity $\times$ trait matrix will become stable: new data adding new rows for entities, but not many new columns for traits. In comparison, the sparseness of the species $\times$ trait matrix should decline in the future; new data will mostly contribute to filling the matrix and increasing the number of species with data per trait. This reduced sparseness of the species $x$ traits matrix will systematically improve the applicability of trait data for macroecology and earth system modelling and will facilitate multivariate analyses for an increasing number of traits. In parallel, the number of records per species-trait combination is increasing: between TRY 1 and 5 it already doubled and will further increase in the future. This increasing number of records per species-trait combination will improve data coverage for analyses of intraspecific trait variation and trait-environment relationships accounting for intraspecific variation. It is noteworthy that the matrix will not only become more complete, but the traits will increasingly be able to inform each other. The 'usual suspects' (i.e. the best covered continuous traits) might not be masters of all traits, but they surely will be very useful as baseline traits and provide a background against which other-maybe more influential-traits can be analysed for coverage, representativeness, orthogonality, etc.

\section{1 | Data completeness and representativeness}

Despite unprecedented and continuously growing data coverage, we observe a humbling lack of completeness and representativeness in many aspects. The best species coverage is achieved for categorical traits relevant to determine PFTs commonly used in global vegetation models. For the traits 'woodiness' and 'plant growth form', even full species coverage is within reach, due to the contribution of data from the GIFT database. For the first time, this provides a global baseline for these traits, which are relevant to understand basic patterns of variation for several other traits (Díaz et al., 2016). With this baseline, future analyses will be able to address representativeness in addition to coverage, which will substantially contribute to better understand the global pattern of plant traits relevant for biodiversity and ecosystem function (König et al., 2019).

Most traits directly relevant for ecology and vegetation modelling are characterized by intraspecific variation and trait-environmental relationships; for these traits, completeness at the global scale is impossible and representativeness is challenging. We find that in the current version of the TRY database, these traits are biased against roots, floral traits and dead organic material, like litter or coarse woody debris. Plant growth forms and phylogeny are generally well represented, but there are significant biases for individual traits. The global distribution of species richness is only marginally reflected by trait measurements. We observe a general bias towards temperate biomes. In contrast to Jetz et al. (2016), the tropics do not stand out as especially underrepresented in our analysis; apart from Europe, all continents contain major regions that are very sparsely represented in TRY.

So far we addressed representativeness in a geographic context only based on species richness, the number of species observed in an ecoregion. To address representativeness in an ecologically more meaningful context, species identity and species abundance should be taken into account. Both aspects are relevant to community attributes and ecosystem function. There is ample evidence in the literature of the high influence, at the level of community structure and ecosystem dynamics, of species that represent a large proportion of the total local biomass (consisting of large individuals and/ or large total cover). Such species have a particularly large impact on the community weighted mean trait value (Garnier et al., 2004), and particularly large individual trees may overrule remaining trees' attributes (Ali et al., 2019). Initial evidence indicates that abundant species are better covered in TRY than rare species. Bruelheide et al. (2019) show that the $25 \%$ most dominant or most frequent species 
compiled in the sPlot vegetation-plot database are better represented by trait data in the TRY database than species observed on the plots overall. We also checked this for the 227 hyperdominant species of the Amazonia tree flora identified by ter Steege et al. (2013). After the consolidation of species names via TNRS, all 227 hyperdominant species are present in the TRY database, with on average 69 traits per species, which is far above average (see Figure 3e). We therefore conclude that the coverage of trait data in TRY is biased towards the more abundant species in the respective ecoregions-which is reasonable and welcome for many kinds of analyses.

We have reported that intraspecific variation in space is in creasingly well covered, but variation in time is hard to estimate. Nevertheless, intraspecific variation in time is relevant for several traits to characterize the seasonal variation of plant and ecosystem function (Xu \& Baldocchi, 2003; Xu \& Griffin, 2006) and long-term trends to inform policy about biodiversity change (Kissling et al., 2018). About half of the geo-referenced trait records have information on the sampling date that could be standardized to year, month and day, but systematic replicates over time ('time series') are rare (but see e.g. the 'Photosynthesis Traits Database'; Xu \& Baldocchi, 2003). In principle, 'non-time series' data allow detection of trait changes over time (Craine et al., 2018), but these analyses are very challenging, as most traits demonstrate stronger variation in geographic space along climate and soil gradients than over time. In addition, the variations of traits on different time scales (diurnal, seasonal, inter-annual variation and long-term trends) are superimposed and hard to disentangle. Apart from this, there is a need to collect and report repeated trait measurements from the same location or population to monitor biodiversity change and inform policy, for example in the context of GEO BON (Kissling et al., 2018).

\subsection{Ways forward}

Figure 1 shows the most obvious way to mobilize additional trait data: the TRY initiative should regularly send calls for data contribution to the wider scientific community, that is the network of more than 6,000 researchers contributing and using trait data via TRY. These calls should be combined with regular publications of respective reference papers. This can be combined with (a) a systematic collection of data sets from public data repositories, which is becoming more effective with the general move by many journals to require that authors make their data open access; and (b) systematic extraction of trait data from the ecological literature, floras and herbarium specimens, which is a promising task, especially for its potential to open a window into the past. In parallel, TRY should further support the 'feed-forward data integration loop' outlined above: using trait data via TRY, identifying gaps, mobilizing and/or measuring new data, contributing additional data to TRY. This has proven very effective for focused data mobilization. If relevant gaps are detected, TRY can also send specific calls to the community.

As TRY has been designed as a community cyber-infrastructure based on the idea of incentive-driven data sharing (Kattge, Díaz, \&
Wirth, 2014), the collaboration and data exchange with other plant trait databases will continue to be the key to achieve a comprehensive representation of plant traits. TRY is, therefore, collaborating with many more recent trait database initiatives, such as, for example, FRED, GIFT, BIEN and the Tundra Trait Team, and since the early days of TRY-GLOPNET, LEDA, SID, BiolFlor, BIOPOP, BROT, the Ecological Flora of the British Isles, eHALOPH, USDA PLANTSdata, BRIDGE and many others. Importantly, these collaborations need to provide mutual benefit. Based on these collaborations, the TRY database may serve as a central node for plant traits in an overarching network of trait databases, currently emerging in the context of the Open Traits Network (Gallagher et al., in press). Finally, new techniques and approaches are gradually becoming available, which may substantially change how plant trait data are collected: remote sensing, citizen sciences, microbiological and molecular screening, etc.

\subsection{Towards a third generation of plant trait data integration and sharing}

We expect that the combination of (a) systematic involvement of the TRY network towards extraction and mobilization of legacy and recent trait data from public repositories, ecological literature, floras and herbaria, (b) facilitation of the 'feed-forward data integration loop' and (c) intensified collaboration of all plant trait-related initiatives, including new approaches and techniques, will be effective towards an increasingly comprehensive representation of plant traits. After the development of integrated databases focused on specific regions or topics, and the development of a 'database of databases', such a joined effort might be leading towards a third generation of plant trait data integration and sharing.

\section{5 | CONCLUSION}

TRY has received institutional support since 2007 and is still growing considerably in quantity and quality. While TRY may be considered a success and potentially a role model for database initiatives, it is important to realize that this development needed time and patience. It took until 2011 for the first TRY publications to appear because the early years of TRY were mostly devoted to the development of the database, organizing the community process towards a joint data sharing policy and building trust. This process involved initially dozens and later hundreds of scientist when it came to agree on moving towards open access. These dynamics do not fit into 3 year funding cycles as typically offered by national funding agencies. A key lesson of TRY is that the development of a database that is trusted by the community and accepted for its service and quality also needs the trust of the funders, that is long-term support, at the scale of decades rather than years. It also needs journals that are willing to accept long author lists and extended references lists to adequately acknowledge the original contributions that are the building blocks of communal databases. 


\section{ACKNOWLEDGEMENTS}

We would like to thank Stephen Long for the invitation to contribute to the special issue celebrating the 25th anniversary of Global Change Biology and the Executive Editor Rachel Shekar for her extraordinary support and patience handling this manuscript. We also thank the publisher for excellent support. We thank the two anonymous reviewers for valuable suggestions, which helped to substantially improve the manuscript. The TRY database is hosted, developed and maintained at the Max Planck Institute for Biogeochemistry (MPI-BGC) in Jena, Germany, in collaboration with the German Centre for Integrative Biodiversity Research (iDiv) Halle-Jena-Leipzig. The TRY database receives additional funding by the Max Planck Society via the Max Planck Fellow Program for Christian Wirth. In addition, the TRY initiative has been supported by the International Programme of Biodiversity Science (DIVERSITAS), the International Geosphere-Biosphere Programme (IGBP), Future Earth, the French Foundation for Biodiversity Research (FRB), and GIS 'Climat, Environnement et Société' France. The TRY initiative is grateful for major support by Linda Maack and the IT Department at the MPIBGC. We would like to thank all data contributors to TRY, who are not co-authors of this paper, that is Pierre Meerts, Jennifer Powers, Nina Koele, Henrik Balslev, John Briggs, Michael White and Robin Chazdon. V.O. thanks RSF (\#19-14-00038). S.D. thanks CONICET, FONCyT and IAI. Finally, the TRY initiative is very grateful to all the numerous scientists not mentioned here measuring plant traits: without their work the TRY database would not exist.

\section{CONFLICT OF INTEREST}

All authors declare no conflict of interest.

\section{AUTHOR CONTRIBUTION}

Jens Kattge, Gerhard Bönisch, Sandra Díaz, Sandra Lavorel, lain Colin Prentice, Paul Leadley and Christian Wirth developed the concept and draft manuscript. Susanne Tautenhahn and Gijsbert Werner contributed analyses and plots for Figures 9, 10 and 11. The other authors contributed plant trait data and/or supported data curation and analysis. All 729 authors contributed to writing.

\section{ORCID}

Jens Kattge (iD https://orcid.org/0000-0002-1022-8469 Sandra Díaz iD https://orcid.org/0000-0003-0012-4612 Susanne Tautenhahn (iD https://orcid.org/0000-0002-2753-3443 Gijsbert D. A. Werner iD https://orcid.org/0000-0002-5426-2562 Tuomas Aakala iD https://orcid.org/0000-0003-0160-6410 Mehdi Abedi iD https://orcid.org/0000-0002-1499-0119 Alicia T. R. Acosta iD https://orcid.org/0000-0001-6572-3187 George C. Adamidis (iD https://orcid.org/0000-0001-8704-6623 Masahiro Aiba iD https://orcid.org/0000-0002-5966-1562 Cécile H. Albert iD https://orcid.org/0000-0002-0991-1068 Julio M. Alcántara iD https://orcid.org/0000-0002-8003-7844 Carolina Alcázar C (ID https://orcid.org/0000-0002-9366-8098 Izabela Aleixo (iD https://orcid.org/0000-0001-9220-8965 Hamada Ali iD https://orcid.org/0000-0002-7062-9344 Christian Ammer (iD https://orcid.org/0000-0002-4235-0135
Carolyn Anderson (iD https://orcid.org/0000-0003-4211-5765

Deborah Mattos Guimarães Apgaua iD https://orcid. org/0000-0002-6303-6989

Tia-Lynn Ashman (D) https://orcid.org/0000-0002-9884-5954 Gregory P. Asner (iD https://orcid.org/0000-0001-7893-6421 Michael Aspinwall iD https://orcid.org/0000-0003-0199-2972 Owen Atkin (iD https://orcid.org/0000-0003-1041-5202 Isabelle Aubin (iD https://orcid.org/0000-0002-5953-1012 Lars Baastrup-Spohr iD https://orcid.org/0000-0001-8382-984X Khadijeh Bahalkeh iD https://orcid.org/0000-0003-1485-0316 Michael Bahn (iD https://orcid.org/0000-0001-7482-9776 William J. Baker iD https://orcid.org/0000-0001-6727-1831 Jan P. Bakker (iD https://orcid.org/0000-0001-7475-5906 Dennis Baldocchi iD https://orcid.org/0000-0003-3496-4919 Jennifer Baltzer iD https://orcid.org/0000-0001-7476-5928 Jos Barlow iD https://orcid.org/0000-0003-4992-2594 Diego R. Barneche iD https://orcid.org/0000-0002-4568-2362 Zdravko Baruch iD https://orcid.org/0000-0002-7264-4812 Denis Bastianelli iD https://orcid.org/0000-0002-6394-5920 John Battles iD https://orcid.org/0000-0001-7124-7893 William Bauerle iD https://orcid.org/0000-0003-3090-234X Marijn Bauters (iD https://orcid.org/0000-0003-0978-6639 Michael Beckmann iD https://orcid.org/0000-0002-5678-265X Hans Beeckman iD https://orcid.org/0000-0001-8954-6277 Carl Beierkuhnlein (iD https://orcid.org/0000-0002-6456-4628 Gavin Belfry iD https://orcid.org/0000-0003-3405-5950 Michael Belluau (iD https://orcid.org/0000-0001-6707-546X Mirela Beloiu (D) https://orcid.org/0000-0002-3592-8170 Raquel Benavides (iD https://orcid.org/0000-0003-2328-5371 Lahcen Benomar (iD https://orcid.org/0000-0001-9301-5655 Mary Lee Berdugo-Lattke iD https://orcid.org/0000-0002-6662-6458 Erika Berenguer (iD https://orcid.org/0000-0001-7357-8805 Rodrigo Bergamin (iD https://orcid.org/0000-0002-2405-9977 Joana Bergmann (iD https://orcid.org/0000-0002-2008-4198 Marcos Bergmann Carlucci iD https://orcid.org/0000-0002-5868-7090 Logan Berner (iD https://orcid.org/0000-0001-8947-0479 Markus Bernhardt-Römermann iD https://orcid.org/0000-0002-2740-2304 Christof Bigler (iD https://orcid.org/0000-0003-3757-6356 Anne D. Bjorkman iD https://orcid.org/0000-0003-2174-7800 Carolina Blanco iD https://orcid.org/0000-0002-8959-2633 Benjamin Blonder (iD https://orcid.org/0000-0002-5061-2385 Dana Blumenthal iD https://orcid.org/0000-0001-7496-0766 Kelly T. Bocanegra-González (iD https://orcid.org/0000-0001-7177-5856 Pascal Boeckx iD https://orcid.org/0000-0003-3998-0010 Katrin Böhning-Gaese (iD https://orcid.org/0000-0003-0477-5586 Laura Boisvert-Marsh iD https://orcid.org/0000-0002-0939-8196 William Bond (iD https://orcid.org/0000-0002-3441-2084 Ben Bond-Lamberty iD https://orcid.org/0000-0001-9525-4633 Arnoud Boom (iD https://orcid.org/0000-0003-1299-691X Coline C. F. Boonman iD https://orcid.org/0000-0003-2417-1579 Kauane Bordin (iD https://orcid.org/0000-0003-3871-6293 Elizabeth H. Boughton (iD https://orcid.org/0000-0003-0932-280X Vanessa Boukili (iD https://orcid.org/0000-0002-5950-2123 
Sandra Bravo iD https://orcid.org/0000-0001-6098-7255 Marco Richard Brendel ID https://orcid.org/0000-0003-4356-4597 Martin R. Broadley iD https://orcid.org/0000-0003-3964-7226 Kerry A. Brown iD https://orcid.org/0000-0002-6342-8966 Helge Bruelheide (iD https://orcid.org/0000-0003-3135-0356 Federico Brumnich iD https://orcid.org/0000-0002-0502-6694 Hans Henrik Bruun (iD https://orcid.org/0000-0003-0674-2577 David Bruy (iD https://orcid.org/0000-0002-7339-0375 Solveig Franziska Bucher iD https://orcid.org/0000-0002-2303-4583 Nina Buchmann iD https://orcid.org/0000-0003-0826-2980 Robert Buitenwerf (iD https://orcid.org/0000-0003-3356-2301 Daniel E. Bunker (iD https://orcid.org/0000-0002-7234-3403 Sabina Burrascano iD https://orcid.org/0000-0002-6537-3313 David F. R. P. Burslem iD https://orcid.org/0000-0001-6033-0990 Bradley J. Butterfield (iD https://orcid.org/0000-0003-0974-9811 Chaeho Byun (iD https://orcid.org/0000-0003-3209-3275 Marcia Marques iD https://orcid.org/0000-0002-1003-9596 Marina C. Scalon iD https://orcid.org/0000-0003-2069-8226 Marco Caccianiga iD https://orcid.org/0000-0001-9715-1830 Marc Cadotte iD https://orcid.org/0000-0002-5816-7693 Maxime Cailleret iD https://orcid.org/0000-0001-6561-1943 James Camac (iD https://orcid.org/0000-0003-4785-0742 Jesús Julio Camarero (iD https://orcid.org/0000-0003-2436-2922 Giandiego Campetella iD https://orcid.org/0000-0001-6126-522X Juan Antonio Campos (iD https://orcid.org/0000-0001-5992-2753 Roberto Canullo iD https://orcid.org/0000-0002-9913-6981 Michele Carbognani iD https://orcid.org/0000-0001-7701-9859 Fabio Carvalho iD https://orcid.org/0000-0002-6305-5602 Fernando Casanoves (iD https://orcid.org/0000-0001-8765-9382 Bastien Castagneyrol iD https://orcid.org/0000-0001-8795-7806 Jane A. Catford iD https://orcid.org/0000-0003-0582-5960 Jeannine Cavender-Bares (D) https://orcid.org/0000-0003-3375-9630 Bruno E. L. Cerabolini iD https://orcid.org/0000-0002-3793-0733 Marco Cervellini (iD https://orcid.org/0000-0002-0853-2330 Eduardo Chacón-Madrigal (iD https://orcid.org/0000-0002-8328-5456 Kenneth Chapin (iD https://orcid.org/0000-0002-8382-4050 F. Stuart Chapin (iD https://orcid.org/0000-0002-2558-9910 Stefano Chelli iD https://orcid.org/0000-0001-7184-8242 Si-Chong Chen (iD https://orcid.org/0000-0002-6855-2595 Anping Chen (D) https://orcid.org/0000-0003-2085-3863 Paolo Cherubini iD https://orcid.org/0000-0002-9809-250X Francesco Chianucci (iD https://orcid.org/0000-0002-5688-2060 Brendan Choat iD https://orcid.org/0000-0002-9105-640X Kyong-Sook Chung (iD https://orcid.org/0000-0002-4464-4698 Milan Chytrý (iD https://orcid.org/0000-0002-8122-3075 Daniela Ciccarelli iD https://orcid.org/0000-0001-9715-9779 Luisa Conti iD https://orcid.org/0000-0001-8047-1467 David Coomes (iD https://orcid.org/0000-0002-8261-2582 Johannes H. C. Cornelissen (iD https://orcid.org/0000-0002-2346-1585 William K. Cornwell iD https://orcid.org/0000-0003-4080-4073 Piermaria Corona iD https://orcid.org/0000-0002-8105-0792 Joseph Craine iD https://orcid.org/0000-0001-6561-3244 Dylan Craven iD https://orcid.org/0000-0003-3940-833X
Anikó Csecserits iD https://orcid.org/0000-0002-0538-4520 Katarina Cufar (iD https://orcid.org/0000-0002-7403-3994 Matthias Cuntz iD https://orcid.org/0000-0002-5966-1829 Ana Carolina da Silva iD https://orcid.org/0000-0002-1285-640X Kyla M. Dahlin (iD https://orcid.org/0000-0002-6016-2605 Matteo Dainese (iD https://orcid.org/0000-0001-7052-5572 Igor Dalke iD https://orcid.org/0000-0001-5711-9916 Michele Dalle Fratte iD https://orcid.org/0000-0002-7907-1586 Anh Tuan Dang-Le iD https://orcid.org/0000-0002-9794-0669 Jirí Danihelka iD https://orcid.org/0000-0002-2640-7867 Masako Dannoura (iD https://orcid.org/0000-0003-0389-871X Samantha Dawson (iD https://orcid.org/0000-0001-5165-9591 Angel De Frutos (iD https://orcid.org/0000-0002-5250-9488 Jonathan R. De Long iD https://orcid.org/0000-0002-7951-4818 Benjamin Dechant (iD https://orcid.org/0000-0001-5171-2364 Nicolas Delpierre iD https://orcid.org/0000-0003-0906-9402 Géraldine Derroire iD https://orcid.org/0000-0001-7239-2881 Arildo S. Dias (iD https://orcid.org/0000-0002-5495-3435 Milton Hugo Diaz-Toribio iD https://orcid.org/0000-0003-1675-2699 Panayiotis G. Dimitrakopoulos iD https://orcid.org/0000-0002-8374-4392 Mark Dobrowolski iD https://orcid.org/0000-0001-5586-4023 Pavel Dřevojan (iD https://orcid.org/0000-0003-0802-3509 Ning Dong (iD https://orcid.org/0000-0003-0793-8854 Leandro Duarte iD https://orcid.org/0000-0003-1771-0407 Stefan Dullinger iD https://orcid.org/0000-0003-3919-0887 Walter Durka iD https://orcid.org/0000-0002-6611-2246 Olga Dymova iD https://orcid.org/0000-0003-2008-6350 Anna E-Vojtkó (D) https://orcid.org/0000-0001-6370-680X Rolf Lutz Eckstein (iD https://orcid.org/0000-0002-6953-3855 Hamid Ejtehadi iD https://orcid.org/0000-0002-6128-2481 James Elser (iD https://orcid.org/0000-0002-1460-2155 Thaise Emilio (iD https://orcid.org/0000-0001-5415-1822 Kristine Engemann (iD https://orcid.org/0000-0003-1431-1726 Mohammad Bagher Erfanian (iD https://orcid.org/0000-0002-8671-9037 Adriane Esquivel-Muelbert (iD https://orcid.org/0000-0001-5335-1259 Marc Estiarte iD https://orcid.org/0000-0003-1176-8480 Tomas F. Domingues (D) https://orcid.org/0000-0003-2857-9838 William F. Fagan iD https://orcid.org/0000-0003-2433-9052 Jaime Fagúndez (iD https://orcid.org/0000-0001-6605-7278 Daniel S. Falster iD https://orcid.org/0000-0002-9814-092X Ying Fan (iD https://orcid.org/0000-0002-0024-7965 Emmanuele Farris (iD https://orcid.org/0000-0002-9843-5998 Fatih Fazlioglu iD https://orcid.org/0000-0002-4723-3640 Yanhao Feng (iD https://orcid.org/0000-0003-0460-4883 Fernando Fernandez-Mendez (D) https://orcid.org/0000-0001-8693-790X Joice Ferreira (iD https://orcid.org/0000-0002-4008-2341 Alessandra Fidelis iD https://orcid.org/0000-0001-9545-2285 Jennifer Firn (iD https://orcid.org/0000-0001-6026-8912 Timothy J. Flowers (D) https://orcid.org/0000-0002-2712-9504 Dan F. B. Flynn iD https://orcid.org/0000-0002-2978-5257 Estelle Forey iD https://orcid.org/0000-0001-6082-3023 Cristiane Forgiarini iD https://orcid.org/0000-0002-0319-7430 Louis François iD https://orcid.org/0000-0001-8292-8360 
Marcelo Frangipani iD https://orcid.org/0000-0001-8050-8282

Grégoire T. Freschet iD https://orcid.org/0000-0002-8830-3860

Ellen L. Fry iD https://orcid.org/0000-0001-7513-2006

Nikolaos M. Fyllas (iD https://orcid.org/0000-0002-5651-5578

Guilherme G. Mazzochini iD https://orcid.org/0000-0002-6932-8544 Rachael Gallagher (iD https://orcid.org/0000-0002-4680-8115 Pablo García-Palacios (iD https://orcid.org/0000-0002-6367-4761 Verónica Gargaglione iD https://orcid.org/0000-0002-0797-1868 Eric Garnier (D) https://orcid.org/0000-0002-9392-5154 Jose Luis Garrido (iD https://orcid.org/0000-0002-6859-4234 André Luís de Gasper iD https://orcid.org/0000-0002-1940-9581 Guillermo Gea-Izquierdo iD https://orcid.org/0000-0003-0148-3721 David Gibson (iD) https://orcid.org/0000-0002-0308-7506 Andrew N. Gillison iD https://orcid.org/0000-0003-2781-1500 Aelton Giroldo iD https://orcid.org/0000-0001-7850-0011 Sean Gleason (iD https://orcid.org/0000-0002-5607-4741 Mariana Gliesch iD https://orcid.org/0000-0003-3226-6762 Erika Gonzalez-Akre iD https://orcid.org/0000-0001-8305-6672 Jose L. Gonzalez-Andujar (iD https://orcid.org/0000-0003-2356-4098 Ana González-Robles iD https://orcid.org/0000-0003-3135-8905 Bente Jessen Graae iD https://orcid.org/0000-0002-5568-4759 Elena Granda iD https://orcid.org/0000-0002-9559-4213 Sarah Graves iD https://orcid.org/0000-0003-3805-4242 Thomas Gregor iD https://orcid.org/0000-0001-7692-8348 Nicolas Gross iD https://orcid.org/0000-0001-9730-3240 Greg R. Guerin (D) https://orcid.org/0000-0002-2104-6695 Alvaro G. Gutiérrez (ID) https://orcid.org/0000-0001-8928-3198 Lillie Haddock (DD https://orcid.org/0000-0003-3432-6191 Jefferson Hall (iD https://orcid.org/0000-0003-4761-9268 Wenxuan Han iD https://orcid.org/0000-0001-8678-7147 Sandy P. Harrison iD https://orcid.org/0000-0001-5687-1903 Joseph E. Hawes (iD https://orcid.org/0000-0003-0053-2018 Pengcheng He iD https://orcid.org/0000-0001-9531-531X Jacob Mason Heberling (iD https://orcid.org/0000-0003-0756-5090 Aveliina Helm (iD https://orcid.org/0000-0003-2338-4564 Bruno Hérault iD https://orcid.org/0000-0002-6950-7286 Ana-Maria Hereş (DD https://orcid.org/0000-0002-1839-1770 Katharina Herz iD https://orcid.org/0000-0001-5676-3646 Myriam Heuertz iD https://orcid.org/0000-0002-6322-3645 Peter Hietz (iD https://orcid.org/0000-0002-0458-6593 Pedro Higuchi (iD https://orcid.org/0000-0002-3855-555X Andrew L. Hipp (iD https://orcid.org/0000-0002-1241-9904 Maria Hock iD https://orcid.org/0000-0001-8401-7309 James Aaron Hogan iD https://orcid.org/0000-0001-9806-3074 Karen Holl iD https://orcid.org/0000-0003-2893-6161 Olivier Honnay iD https://orcid.org/0000-0002-4287-8511 Enqing Hou iD https://orcid.org/0000-0003-4864-2347 Nate Hough-Snee (iD https://orcid.org/0000-0003-4581-0931 Knut Anders Hovstad iD https://orcid.org/0000-0002-7108-0787 Tomoaki Ichie iD https://orcid.org/0000-0002-1979-5806 Boris Igić (iD https://orcid.org/0000-0002-6302-8030 Estela Illa iD https://orcid.org/0000-0001-7136-6518 Marney Isaac (iD https://orcid.org/0000-0002-5975-833X
Masae Ishihara (iD https://orcid.org/0000-0002-8365-7766 Leonid Ivanov (iD https://orcid.org/0000-0001-6900-5086 Larissa Ivanova (iD https://orcid.org/0000-0003-2363-9619 Colleen M. Iversen (iD https://orcid.org/0000-0001-8293-3450 Jordi Izquierdo (iD https://orcid.org/0000-0002-3949-1295 Robert B. Jackson (iD https://orcid.org/0000-0001-8846-7147 Benjamin Jackson (iD https://orcid.org/0000-0001-6405-8111 Hervé Jactel iD https://orcid.org/0000-0002-8106-5310 Andrzej M. Jagodzinski (iD https://orcid.org/0000-0001-6899-0985 Steven Jansen iD https://orcid.org/0000-0002-4476-5334 Thomas Jenkins (D) https://orcid.org/0000-0002-3036-971X Anke Jentsch iD https://orcid.org/0000-0002-2345-8300 Guo-Feng Jiang (iD https://orcid.org/0000-0002-3221-8608 Jesper Liengaard Johansen iD https://orcid.org/0000-0001-7602-316X David Johnson (iD https://orcid.org/0000-0003-2299-2525 Eric J. Jokela iD https://orcid.org/0000-0002-1819-1285 Carlos Alfredo Joly iD https://orcid.org/0000-0002-7945-2805 Gregory J. Jordan (DD https://orcid.org/0000-0002-6033-2766 Grant Stuart Joseph iD https://orcid.org/0000-0002-8731-9828 Decky Junaedi iD https://orcid.org/0000-0001-5846-6135 Robert R. Junker (iD https://orcid.org/0000-0002-7919-9678 Eric Justes (iD https://orcid.org/0000-0001-7390-7058 Jeffrey Kane iD https://orcid.org/0000-0003-1453-9608 Zdenek Kaplan (iD https://orcid.org/0000-0003-1707-7461 Teja Kattenborn (iD https://orcid.org/0000-0001-7381-3828 Elizabeth Kearsley (iD https://orcid.org/0000-0003-0046-3606 Anne Kempel iD https://orcid.org/0000-0002-0563-901X Tanaka Kenzo (iD https://orcid.org/0000-0002-4856-7204 Mohammed I. Khalil ID https://orcid.org/0000-0002-9310-2780 Nicole L. Kinlock iD https://orcid.org/0000-0002-2917-5133 Wilm Daniel Kissling iD https://orcid.org/0000-0002-7274-6755 Kaoru Kitajima (iD) https://orcid.org/0000-0001-6822-8536 Thomas Kitzberger (iD https://orcid.org/0000-0002-9754-4121 Rasmus Kjøller (iD https://orcid.org/0000-0002-2027-4119 Tamir Klein iD https://orcid.org/0000-0002-3882-8845 Michael Kleyer (iD https://orcid.org/0000-0002-0824-2974 Jitka Klimešová iD https://orcid.org/0000-0003-0123-3263 Joice Klipel iD https://orcid.org/0000-0003-3936-9692 Brian Kloeppel (iD https://orcid.org/0000-0003-0215-4671 Takashi Kohyama iD https://orcid.org/0000-0001-7186-8585 Fumito Koike iD https://orcid.org/0000-0002-6588-6485 Johannes Kollmann (iD https://orcid.org/0000-0002-4990-3636 Kimberly Komatsu iD https://orcid.org/0000-0001-7056-4547 Christian König (iD https://orcid.org/0000-0003-0585-5983 Nathan J. B. Kraft iD https://orcid.org/0000-0001-8867-7806 Koen Kramer iD https://orcid.org/0000-0002-1402-2775 Holger Kreft iD https://orcid.org/0000-0003-4471-8236 Ingolf Kühn (iD https://orcid.org/0000-0003-1691-8249 Dushan Kumarathunge iD https://orcid.org/0000-0003-1309-4731 Jonas Kuppler (iD https://orcid.org/0000-0003-4409-9367 Hiroko Kurokawa iD https://orcid.org/0000-0001-8778-8045 Yoko Kurosawa iD https://orcid.org/0000-0002-1419-7575 Shem Kuyah iD https://orcid.org/0000-0001-7692-585X 
Jean-Paul Laclau (DD https://orcid.org/0000-0002-2506-214X Eric Lamb (iD https://orcid.org/0000-0001-5201-4541 Andrea Lamprecht (D) https://orcid.org/0000-0002-8719-026X Daniel J. Larkin (D) https://orcid.org/0000-0001-6378-0495 Daniel Laughlin (D) https://orcid.org/0000-0002-9651-5732 Yoann Le Bagousse-Pinguet $\mathbb{D}^{D}$ https://orcid.org/0000-0002-5615-5541 Guerric le Maire (DD https://orcid.org/0000-0002-5227-958X Peter C. le Roux (D) https://orcid.org/0000-0002-7941-7444 Elizabeth le Roux (D) https://orcid.org/0000-0001-8468-8284 Tali Lee (ID https://orcid.org/0000-0002-3933-6607 Frederic Lens (iD https://orcid.org/0000-0002-5001-0149 Simon L. Lewis (D) https://orcid.org/0000-0002-8066-6851

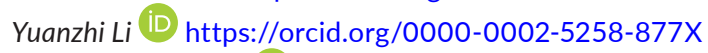
Jeremy W. Lichstein (D) https://orcid.org/0000-0001-5553-6142 Jun Ying Lim (D) https://orcid.org/0000-0001-7493-2159 Juan Carlos Linares (D) https://orcid.org/0000-0001-8375-6353 Daijun Liu (D) https://orcid.org/0000-0002-0993-0832 Udayangani Liu (D) https://orcid.org/0000-0002-6192-1319 Madelon Lohbeck (iD) https://orcid.org/0000-0002-3959-1800 Álvaro López-García (D) https://orcid.org/0000-0001-8267-3572 Gabriela Lopez-Gonzalez (D) https://orcid.org/0000-0002-0814-8830 Zdeňka Lososová (D) https://orcid.org/0000-0001-9152-7462 Frédérique Louault (iD https://orcid.org/0000-0002-9430-4261 Balázs A. Lukács (D) https://orcid.org/0000-0002-3163-1281 Michele Lussu (D) https://orcid.org/0000-0002-1313-4732 Camilla Maciel Rabelo Pereira (iD https://orcid.org/0000-0002-7027-7430 Vincent Maire (D) https://orcid.org/0000-0002-3245-2568 Annikki Mäkelä (D) https://orcid.org/0000-0001-9633-7350 Harri Mäkinen (D) https://orcid.org/0000-0002-1820-6264 Ana Claudia Mendes Malhado (D) https://orcid.org/0000-0003-3621-779X Azim Mallik (D) https://orcid.org/0000-0002-6275-6255 Peter Manning (D) https://orcid.org/0000-0002-7940-2023

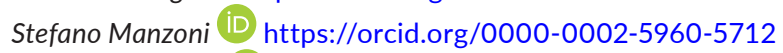
Zuleica Marchetti (D) https://orcid.org/0000-0002-9241-9217 Vinicius Marcilio-Silva (D) https://orcid.org/0000-0002-5276-2838 Eric Marcon (D) https://orcid.org/0000-0002-5249-321X Michela Marignani (DD https://orcid.org/0000-0002-8420-5454 Lars Markesteijn (D) https://orcid.org/0000-0003-3046-3121 Adam Martin (D) https://orcid.org/0000-0002-7207-4004 Cristina Martínez-Garza (D) https://orcid.org/0000-0002-9310-564X Jordi Martínez-Vilalta (D) https://orcid.org/0000-0002-2332-7298 Tereza Mašková (D) https://orcid.org/0000-0001-8386-5146 Kelly Mason (D) https://orcid.org/0000-0003-3426-3178 Itay Mayrose (D) https://orcid.org/0000-0002-8460-1502 James McCarthy (D) https://orcid.org/0000-0003-3060-1678 M. Luke McCormack (D) https://orcid.org/0000-0002-8300-5215 Katherine McCulloh (iD https://orcid.org/0000-0003-0801-3968 lan R. McFadden (D) https://orcid.org/0000-0002-4508-7272 Mara Y. McPartland (D) https://orcid.org/0000-0002-1242-0438 Belinda Medlyn (D) https://orcid.org/0000-0001-5728-9827 Zia Mehrabi (D) https://orcid.org/0000-0001-9574-0420 Patrick Meir (D) https://orcid.org/0000-0002-2362-0398 Felipe P. L. Melo (D) https://orcid.org/0000-0002-1271-3214
Maurizio Mencuccini (D) https://orcid.org/0000-0003-0840-1477 Céline Meredieu (D) https://orcid.org/0000-0002-2950-2253 Julie Messier (D) https://orcid.org/0000-0003-1637-6793 Sean T. Michaletz (iD) https://orcid.org/0000-0003-2158-6525 Chrysanthi Michelaki iD https://orcid.org/0000-0001-9217-0063 Ruben Milla iD https://orcid.org/0000-0001-8912-4373 Jesse E. D. Miller (D) https://orcid.org/0000-0002-2316-779X Vanessa Minden (D) https://orcid.org/0000-0002-4933-5931 Ray Ming (D) https://orcid.org/0000-0002-9417-5789 Karel Mokany (D) https://orcid.org/0000-0003-4199-3697 Angela T. Moles (D) https://orcid.org/0000-0003-2041-7762 Attila Molnár (iD https://orcid.org/0000-0001-7096-9579 Jane Molofsky (D) https://orcid.org/0000-0001-7927-516X Arnaud Monty (D) https://orcid.org/0000-0003-1910-6748 Alvaro Moreno-Martínez (iD https://orcid.org/0000-0003-2990-7768 Marco Moretti https://orcid.org/0000-0002-5845-3198 Akira S. Mori (D) https://orcid.org/0000-0002-8422-1198 Shigeta Mori (D) https://orcid.org/0000-0001-5604-2443 Dave Morris (D) https://orcid.org/0000-0002-5739-0594 Jane Morrison (iD https://orcid.org/0000-0002-4055-4679 Ladislav Mucina (iD) https://orcid.org/0000-0003-0317-8886 Christopher D. Muir (D) https://orcid.org/0000-0003-2555-3878 Sandra Cristina Müller (D) https://orcid.org/0000-0002-6316-2897

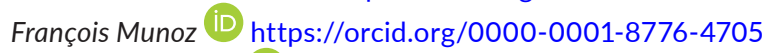
Isla H. Myers-Smith (D) https://orcid.org/0000-0002-8417-6112 Balachandran Natesan (D) https://orcid.org/0000-0001-6396-3865 Luka Negoita (D) https://orcid.org/0000-0003-3225-7575 Andrew S. Nelson (D) https://orcid.org/0000-0001-8715-7825 Eike Lena Neuschulz (iD) https://orcid.org/0000-0001-7526-2580 Jian Ni ${ }^{(D)}$ https://orcid.org/0000-0001-5411-7050 Georg Niedrist (D) https://orcid.org/0000-0002-7511-6273 Ülo Niinemets (D) https://orcid.org/0000-0002-3078-2192 Rachael Nolan (D) https://orcid.org/0000-0001-9277-5142 Henning Nottebrock (iD) https://orcid.org/0000-0003-2238-2700 Yann Nouvellon (iD) https://orcid.org/0000-0003-1920-3847 Alexander Novakovskiy (D) https://orcid.org/0000-0003-4105-7436 Kristin Odden Nystuen (iD https://orcid.org/0000-0003-1027-469X Kevin O'Hara (D) https://orcid.org/0000-0002-3800-9188 Andrew O'Reilly-Nugent (iD https://orcid.org/0000-0003-2071-6279 Simon Oakley (D) https://orcid.org/0000-0002-5757-7420 Walter Oberhuber (D) https://orcid.org/0000-0002-5197-7044 Toshiyuki Ohtsuka (D) https://orcid.org/0000-0002-9032-7933 Ricardo Oliveira (D) https://orcid.org/0000-0001-8044-3458 Kinga Öllerer (D) https://orcid.org/0000-0003-3142-0000 Mark E. Olson (D) https://orcid.org/0000-0003-3715-4567 Vladimir Onipchenko iD https://orcid.org/0000-0002-1626-1171 Yusuke Onoda (D) https://orcid.org/0000-0001-6245-2342 Renske E. Onstein (D) https://orcid.org/0000-0002-2295-3510 Ivika Ostonen (D) https://orcid.org/0000-0001-9043-6083 Gianluigi Ottaviani (D) https://orcid.org/0000-0003-3027-4638 Sarah Otto (D) https://orcid.org/0000-0003-3042-0818 Gerhard E. Overbeck (iD https://orcid.org/0000-0002-8716-5136 Wim A. Ozinga (D) https://orcid.org/0000-0002-6369-7859 
C. E. Timothy Paine iD https://orcid.org/0000-0001-8705-3719 Robin J. Pakeman (iD https://orcid.org/0000-0001-6248-4133 Aristotelis C. Papageorgiou (iD https://orcid.org/0000-0001-6657-7820 Meelis Pärtel (DD https://orcid.org/0000-0002-5874-0138 Susana Paula (iD https://orcid.org/0000-0001-5405-6155 Juraj Paule iD https://orcid.org/0000-0001-5375-7689 Harald Pauli iD https://orcid.org/0000-0002-9842-9934 Juli G. Pausas (iD https://orcid.org/0000-0003-3533-5786 Begoña Peco (iD https://orcid.org/0000-0003-2149-1438 Josep Penuelas iD https://orcid.org/0000-0002-7215-0150 Antonio Perea iD https://orcid.org/0000-0001-8351-9358 Pablo Peri (D) https://orcid.org/0000-0002-5398-4408 Ana Carolina Petisco-Souza iD https://orcid.org/0000-0002-2393-6210 Alessandro Petraglia iD https://orcid.org/0000-0003-4632-2251 Any Mary Petritan iD https://orcid.org/0000-0003-3683-1108 Oliver L. Phillips (iD https://orcid.org/0000-0002-8993-6168 Simon Pierce (iD https://orcid.org/0000-0003-1182-987X Valério D. Pillar iD https://orcid.org/0000-0001-6408-2891 Jan Pisek (iD https://orcid.org/0000-0003-0396-2072 Hendrik Poorter (iD https://orcid.org/0000-0001-9900-2433 Angelika Portsmuth (iD https://orcid.org/0000-0002-5100-2888 Peter Poschlod iD https://orcid.org/0000-0003-4473-7656 A. Shafer Powell iD https://orcid.org/0000-0002-9622-0061 Sally A. Power (iD https://orcid.org/0000-0002-2723-8671 Giacomo Puglielli iD https://orcid.org/0000-0003-0085-4535 Petr Pyšek iD https://orcid.org/0000-0001-8500-442X Valerie Raevel ID https://orcid.org/0000-0002-9631-114X Courtenay A. Ray (iD https://orcid.org/0000-0002-2276-5915 Peter B. Reich iD http://orcid.org/0000-0003-4424-662X Douglas E. B. Reid (iD https://orcid.org/0000-0002-0967-5122 Maxime Réjou-Méchain (D) https://orcid.org/0000-0003-2824-267X Victor Resco de Dios (iD https://orcid.org/0000-0002-5721-1656 Sabina Ribeiro (iD https://orcid.org/0000-0002-4504-3050 Sarah Richardson (iD https://orcid.org/0000-0002-4097-0381 Matthias C. Rillig (iD https://orcid.org/0000-0003-3541-7853 Fiamma Riviera (iD https://orcid.org/0000-0003-2505-8180 Elisabeth M. R. Robert (iD https://orcid.org/0000-0002-3611-7265 Bjorn Robroek (iD https://orcid.org/0000-0002-6714-0652 Adam Roddy (iD https://orcid.org/0000-0002-4423-8729 Arthur Vinicius Rodrigues (D) https://orcid.org/0000-0003-2656-558X Alistair Rogers (iD https://orcid.org/0000-0001-9262-7430 Emily Rollinson (iD https://orcid.org/0000-0003-3428-2352 Victor Rolo iD https://orcid.org/0000-0001-5854-9512 Christine Römermann (iD https://orcid.org/0000-0003-3471-0951 Dina Ronzhina (iD https://orcid.org/0000-0003-0854-0223 Christiane Roscher (iD https://orcid.org/0000-0001-9301-7909 Julieta A. Rosell (D) https://orcid.org/0000-0001-5741-8027 Milena Fermina Rosenfield (D) https://orcid.org/0000-0002-1799-2822 David B. Roy iD https://orcid.org/0000-0002-5147-0331 Samuel Royer-Tardif (D) https://orcid.org/0000-0001-7809-6485 Nadja Rüger (iD) https://orcid.org/0000-0003-2371-4172 Ricardo Ruiz-Peinado iD https://orcid.org/0000-0003-0126-1651 Sabine B. Rumpf iD https://orcid.org/0000-0001-5909-9568
Masahiro Ryo iD https://orcid.org/0000-0002-5271-3446 Lawren Sack iD https://orcid.org/0000-0002-7009-7202 Beatriz Salgado-Negret iD https://orcid.org/0000-0002-3103-9878 Roberto Salguero-Gomez iD https://orcid.org/0000-0002-6085-4433 Ignacio Santa-Regina iD https://orcid.org/0000-0002-7312-3605 Ana Carolina Santacruz-García (iD https://orcid.org/0000-0002-3340-4859 Joaquim Santos (iD https://orcid.org/0000-0002-2160-4968 Jordi Sardans (iD https://orcid.org/0000-0003-2478-0219 Brandon Schamp iD https://orcid.org/0000-0002-6885-2029 Michael Scherer-Lorenzen (iD https://orcid.org/0000-0001-9566-590X Matthias Schleuning iD https://orcid.org/0000-0001-9426-045X Bernhard Schmid iD https://orcid.org/0000-0002-8430-3214 Marco Schmidt (iD https://orcid.org/0000-0001-6087-6117 Sylvain Schmitt iD https://orcid.org/0000-0001-7759-7106 Julio V. Schneider (iD https://orcid.org/0000-0002-9823-6569 Simon D. Schowanek iD https://orcid.org/0000-0001-6036-7507 Julian Schrader iD https://orcid.org/0000-0002-8392-211X Franziska Schrodt iD https://orcid.org/0000-0001-9053-8872 Frank Schurr iD https://orcid.org/0000-0002-4518-0602 Galia Selaya Garvizu (DD https://orcid.org/0000-0003-1920-7450 Marina Semchenko (iD https://orcid.org/0000-0001-6196-3562 Julia C. Sfair iD https://orcid.org/0000-0003-3823-7233 Christine S. Sheppard iD https://orcid.org/0000-0002-7847-2310 Serge Sheremetiev iD https://orcid.org/0000-0002-0318-6766 Satomi Shiodera (iD https://orcid.org/0000-0002-6849-8568 Bill Shipley iD https://orcid.org/0000-0002-7026-3880 Tanvir Ahmed Shovon iD https://orcid.org/0000-0003-3311-1797 Alrun Siebenkäs iD https://orcid.org/0000-0001-9676-7094 Carlos Sierra iD https://orcid.org/0000-0003-0009-4169 Vasco Silva iD https://orcid.org/0000-0003-2729-1824 Mateus Silva iD https://orcid.org/0000-0002-4281-3400 Tommaso Sitzia (D) https://orcid.org/0000-0001-6221-4256 Henrik Sjöman (iD https://orcid.org/0000-0002-5526-6303 Martijn Slot iD https://orcid.org/0000-0002-5558-1792 Nicholas G. Smith iD https://orcid.org/0000-0001-7048-4387 Darwin Sodhi (iD https://orcid.org/0000-0003-2119-2681 Pamela Soltis (iD https://orcid.org/0000-0001-9310-8659 Douglas Soltis iD https://orcid.org/0000-0001-8638-4137 Ben Somers (iD https://orcid.org/0000-0002-7875-107X Grégory Sonnier (iD https://orcid.org/0000-0002-0614-7033 Mia Vedel Sørensen (iD https://orcid.org/0000-0002-2225-6117 Enio Egon Sosinski iD https://orcid.org/0000-0001-6310-9474 Nadejda A. Soudzilovskaia (iD https://orcid.org/0000-0002-9584-2109 Alexandre F. Souza iD https://orcid.org/0000-0001-7468-3631 Marko Spasojevic (iD https://orcid.org/0000-0003-1808-0048 Marta Gaia Sperandii iD https://orcid.org/0000-0002-2507-5928 Amanda B. Stan iD https://orcid.org/0000-0001-6111-0578 James Stegen (iD https://orcid.org/0000-0001-9135-7424 Klaus Steinbauer iD https://orcid.org/0000-0002-3730-9920 Jörg G. Stephan (iD https://orcid.org/0000-0001-6195-7867 Dejan B. Stojanovic (D) https://orcid.org/0000-0003-2967-2049 Jens-Christian Svenning iD https://orcid.org/0000-0002-3415-0862 Ivana Svitková (iD https://orcid.org/0000-0002-5671-0330 
Marek Svitok iD https://orcid.org/0000-0003-2710-8102 Miroslav Svoboda iD https://orcid.org/0000-0003-4050-3422 Nathan Swenson iD https://orcid.org/0000-0003-3819-9767 Marcelo Tabarelli iD https://orcid.org/0000-0001-7573-7216 Kentaro Takagi iD https://orcid.org/0000-0002-1321-2841 Ulrike Tappeiner (iD https://orcid.org/0000-0002-0195-7459 Rubén Tarifa iD https://orcid.org/0000-0002-0288-1978 Simon Tauugourdeau iD https://orcid.org/0000-0001-6561-3228 Cagatay Tavsanoglu iD https://orcid.org/0000-0003-4447-6492 Mariska te Beest (iD https://orcid.org/0000-0003-3673-4105 Leho Tedersoo (iD https://orcid.org/0000-0002-1635-1249 Nelson Thiffault iD https://orcid.org/0000-0003-2017-6890 Dominik Thom iD https://orcid.org/0000-0001-8091-6075 Evert Thomas (iD https://orcid.org/0000-0002-7838-6228 Peter E. Thornton (iD https://orcid.org/0000-0002-4759-5158 Wilfried Thuiller iD https://orcid.org/0000-0002-5388-5274 Lubomír Tichý (iD https://orcid.org/0000-0001-8400-7741 David Tissue (D) https://orcid.org/0000-0002-8497-2047 Mark G. Tjoelker (iD https://orcid.org/0000-0003-4607-5238 David Yue Phin Tng (iD https://orcid.org/0000-0001-5135-0922 Joseph Tobias (iD https://orcid.org/0000-0003-2429-6179 Péter Török (iD https://orcid.org/0000-0002-4428-3327 Tonantzin Tarin (ID https://orcid.org/0000-0001-5125-579X José M. Torres-Ruiz iD https://orcid.org/0000-0003-1367-7056 Béla Tóthmérész (iD https://orcid.org/0000-0002-4766-7668 Martina Treurnicht iD https://orcid.org/0000-0002-0853-0247 Franck Trolliet (ID https://orcid.org/0000-0003-1472-2592 Volodymyr Trotsiuk iD https://orcid.org/0000-0002-8363-656X James L. Tsakalos (iD https://orcid.org/0000-0001-5067-196X loannis Tsiripidis (iD https://orcid.org/0000-0001-9373-676X Vladimir Usoltsev (iD http://orcid.org/0000-0003-4587-8952 Matthew Vadeboncoeur (iD https://orcid.org/0000-0002-8269-0708 Fernando Valladares (iD https://orcid.org/0000-0002-5374-4682 Jana Vamosi iD https://orcid.org/0000-0002-2376-0729 Peter M. van Bodegom (iD https://orcid.org/0000-0003-0771-4500 Michiel van Breugel iD https://orcid.org/0000-0003-2778-7803 Elisa Van Cleemput (iD https://orcid.org/0000-0002-5305-9749 Stephni van der Merwe (ID https://orcid.org/0000-0002-6385-8536 Masha T. van der Sande (iD https://orcid.org/0000-0002-6845-2308 Mark van Kleunen iD https://orcid.org/0000-0002-2861-3701 Koenraad Van Meerbeek iD https://orcid.org/0000-0002-9260-3815 Kim André Vanselow (iD https://orcid.org/0000-0003-3299-6220 Laura Varone iD https://orcid.org/0000-0002-9989-5283 Maribel Yesenia Vasquez Valderrama iD https://orcid. org/0000-0001-7839-7229 Kiril Vassilev iD https://orcid.org/0000-0003-4376-5575 Mark Vellend (iD https://orcid.org/0000-0002-2491-956X Erik J. Veneklaas iD https://orcid.org/0000-0002-7030-4056 Hans Verbeeck iD https://orcid.org/0000-0003-1490-0168 Kris Verheyen (iD https://orcid.org/0000-0002-2067-9108 Alexander Vibrans (iD https://orcid.org/0000-0002-8789-5833 Ima Vieira iD https://orcid.org/0000-0003-1233-318X Jaime Villacís iD https://orcid.org/0000-0001-7752-8506
Cyrille Violle iD https://orcid.org/0000-0002-2471-9226 Katrin Wagner iD https://orcid.org/0000-0003-4326-1086 Anthony P. Walker iD https://orcid.org/0000-0003-0557-5594 Martyn Waller (iD https://orcid.org/0000-0003-3876-2492 Han Wang (iD https://orcid.org/0000-0003-2482-1818 Feng Wang (iD https://orcid.org/0000-0003-3595-515X Weiqi Wang (iD https://orcid.org/0000-0001-8503-0978 Harry Watkins iD https://orcid.org/0000-0002-4038-7145 James T. Weedon (D) https://orcid.org/0000-0003-0491-8719 Liping Wei (iD https://orcid.org/0000-0002-4296-9851 Patrick Weigelt (iD https://orcid.org/0000-0002-2485-3708 Evan Weiher (iD https://orcid.org/0000-0002-5375-9964 Aidan W. Wells iD https://orcid.org/0000-0002-2209-3282 Camilla Wellstein iD https://orcid.org/0000-0001-6994-274X Mark Westoby iD https://orcid.org/0000-0001-7690-4530 Philip John White iD https://orcid.org/0000-0003-0827-288X Mathew Williams (iD https://orcid.org/0000-0001-6117-5208 Daniel E. Winkler iD https://orcid.org/0000-0003-4825-9073 lan J. Wright iD https://orcid.org/0000-0001-8338-9143 S. Joseph Wright iD https://orcid.org/0000-0003-4260-5676 Justin Wright iD https://orcid.org/0000-0002-9102-5347 Bruno X. Pinho iD https://orcid.org/0000-0002-6588-3575 Fabiano Ximenes iD https://orcid.org/0000-0003-0516-9414 Toshihiro Yamada iD https://orcid.org/0000-0001-9307-6255 Keiko Yamaji iD https://orcid.org/0000-0003-2875-5619 Ruth Yanai (iD) https://orcid.org/0000-0001-6987-2489 Amy E. Zanne ID https://orcid.org/0000-0001-6379-9452 David Zelený (iD https://orcid.org/0000-0001-5157-044X Jingming Zheng (iD https://orcid.org/0000-0002-8517-9999 Ji Zheng (iD https://orcid.org/0000-0003-4936-1168 Kasia Ziemińska iD https://orcid.org/0000-0001-7750-4758 Chad R. Zirbel iD https://orcid.org/0000-0002-9289-1722 Irié Casimir Zo-Bi iD https://orcid.org/0000-0003-0982-8579 Gerhard Zotz iD https://orcid.org/0000-0002-6823-2268

\section{REFERENCES}

Aerts, R., \& Chapin, F. S. (2000). The mineral nutrition of wild plants revisited: A re-evaluation of processes and patterns. Advances in Ecological Research, 30, 1-67. https://doi.org/10.1016/s0065-2504(08)60016-1

Akhmetzhanova, A. A., Soudzilovskaia, N. A., Onipchenko, V. G., Cornwell, W. K., Agafonov, V. A., Selivanov, I. A., \& Cornelissen, J. H. C. (2012). A rediscovered treasure: Mycorrhizal intensity database for 3000 vascular plant species across the former Soviet Union. Ecology, 93(3), 689-690. https://doi.org/10.1890/11-1749.1

Ali, A., Lin, S. L., He, J. K., Kong, F. M., Yu, J. H., \& Jiang, H. S. (2019). Bigsized trees overrule remaining trees' attributes and species richness as determinants of aboveground biomass in tropical forests. Global Change Biology, 25(8), 2810-2824. https://doi.org/10.1111/gcb.14707

Baraloto, C., Timothy Paine, C. E. T., Patino, S., Bonal, D., Herault, B., $\&$ Chave, J. (2010). Functional trait variation and sampling strategies in species-rich plant communities. Functional Ecology, 24(1), 208-216. https://doi.org/10.1111/j.1365-2435.2009.01600.x

Bjorkman, A. D., Myers-Smith, I. H., Elmendorf, S. C., Normand, S., Rüger, N., Beck, P. S. A., ... Weiher, E. (2018). Plant functional trait change across a warming tundra biome. Nature, 562(7725), 57-62. https://doi.org/10.1038/s41586-018-0563-7 
Bjorkman, A. D., Myers-Smith, I. H., Elmendorf, S. C., Normand, S., Thomas, H. J. D., Alatalo, J. M., ... Zamin, T. (2018). Tundra Trait Team: A database of plant traits spanning the tundra biome. Global Ecology and Biogeography, 27(12), 1402-1411. https://doi.org/10.1111/geb.12821

Boeddinghaus, R. S., Marhan, S., Berner, D., Boch, S., Fischer, M., Hölzel, N., ... Manning, P. (2019). Plant functional trait shifts explain concurrent changes in the structure and function of grassland soil microbial communities. Journal of Ecology, 107(5), 2197-2210. https://doi. org/10.1111/1365-2745.13182

Boyle, B., Hopkins, N., Lu, Z., Raygoza Garay, J. A., Mozzherin, D., Rees, T., ... Enquist, B. J. (2013). The taxonomic name resolution service: An online tool for automated standardization of plant names. BMC Bioinformatics, 14(1). https://doi.org/10.1186/1471-2105-14-16

Bruelheide, H., Dengler, J., Jiménez-Alfaro, B., Purschke, O., Hennekens, S. M., Chytrý, M., ... Zverev, A. (2019). sPlot - A new tool for global vegetation analyses. Journal of Vegetation Science, 30(2), 161-186. https://doi.org/10.1111/jvs.12710

Bruelheide, H., Dengler, J., Purschke, O., Lenoir, J., Jiménez-Alfaro, B., Hennekens, S. M., ... Jandt, U. (2018). Global trait-environment relationships of plant communities. Nature Ecology \& Evolution, 2(12), 1906-1917. https://doi.org/10.1038/s41559-018-0699-8

Butler, E. E., Datta, A., Flores-Moreno, H., Chen, M., Wythers, K. R., Fazayeli, F., ... Reich, P. B. (2017). Mapping local and global variability in plant trait distributions. Proceedings of the National Academy of Sciences of the United States of America, 114(51), E10937-E10946. https://doi.org/10.1073/pnas.1708984114

Craine, J. M., Elmore, A. J., Wang, L., Aranibar, J., Bauters, M., Boeckx, P., ... Zmudczyńska-Skarbek, K. (2018). Isotopic evidence for oligotrophication of terrestrial ecosystems. Nature Ecology \& Evolution, 2(11), 1735-1744. https://doi.org/10.1038/s41559-018-0694-0

Craven, D., Eisenhauer, N., Pearse, W. D., Hautier, Y., Isbell, F., Roscher, C., ... Manning, P. (2018). Multiple facets of biodiversity drive the diversity-stability relationship. Nature Ecology \& Evolution, 2(10), 15791587. https://doi.org/10.1038/s41559-018-0647-7

deVries, F. T., Manning, P., Tallowin, J. R. B., Mortimer, S. R., Pilgrim, E. S., Harrison, K. A., ... Bardgett, R. D. (2012). Abiotic drivers and plant traits explain landscape-scale patterns in soil microbial communities. Ecology Letters, 15(11), 1230-1239. https://doi. org/10.1111/j.1461-0248.2012.01844.x

Delgado-Baquerizo, M., Fry, E. L., Eldridge, D. J., deVries, F. T., Manning, P., Hamonts, K., ... Bardgett, R. D. (2018). Plant attributes explain the distribution of soil microbial communities in two contrasting regions of the globe. New Phytologist, 219(2), 574-587. https://doi. org/10.1111/nph.15161

Díaz, S., Hodgson, J. G., Thompson, K., Cabido, M., Cornelissen, J. H. C., Jalili, A., ... Zak, M. R. (2004). The plant traits that drive ecosystems: Evidence from three continents. Journal of Vegetation Science, 15(3), 295-304. https://doi.org/10.1658/1100-9233(2004)015[0295:tpttd e]2.0.co;2

Díaz, S., Kattge, J., Cornelissen, J. H. C., Wright, I. J., Lavorel, S., Dray, S., ... Gorné, L. D. (2016). The global spectrum of plant form and function. Nature, 529(7585), 167-171. https://doi.org/10.1038/nature 16489

Díaz, S., Lavorel, S., de Bello, F., Quétier, F., Grigulis, K., \& Robson, T. M. (2007). Incorporating plant functional diversity effects in ecosystem service assessments. Proceedings of the National Academy of Sciences of the United States of America, 104(52), 20684-20689. https://doi. org/10.1073/pnas.0704716104

Engemann, K., Sandel, B., Boyle, B., Enquist, B. J., Jørgensen, P. M., Kattge, J., ... Svenning, J. C. (2016). A plant growth form dataset for the New World. Ecology, 97(11), 3243-3243. https://doi.org/10.1002/ ecy.1569

Enquist, B., Condit, R., Peet, R., Schildhauer, M., \& Thiers, B. (2016). Cyberinfrastructure for an integrated botanical information network to investigate the ecological impacts of global climate change on plant biodiversity. PeerJ Preprints, 4, e2615v2. https://doi.org/10.7287/ peerj.preprints. $2615 \mathrm{v} 2$

Fan, Y., Miguez-Macho, G., Jobbágy, E. G., Jackson, R. B., \& Otero-Casal, C. (2017). Hydrologic regulation of plant rooting depth. Proceedings of the National Academy of Sciences of the United States of America, 114(40), 10572-10577. https://doi.org/10.1073/pnas.1712381114

Fitter, A. H., \& Peat, H. J. (1994). The ecological flora database. Journal of Ecology, 82(2), 415. https://doi.org/10.2307/2261309

Gallagher, R. V., Falster, D. S., Maitner, B., Salguero-Gomez, R., Vandvik, V., Pearse, W., ... Enquist, B. J. (in press). The open traits network: Using open science principles to accelerate trait-based science across the tree of life. Nature Ecology \& Evolution.

Garnier, E., Cortez, J., Billès, G., Navas, M.-L., Roumet, C., Debussche, M., ... Toussaint, J.-P. (2004). Plant functional markers capture ecosystem properties during secondary succession. Ecology, 85, 2630-2637. https://doi.org/10.1890/03-0799

Garnier, E., \& Navas, M.-L. (2012). A trait-based approach to comparative functional plant ecology: Concepts, methods and applications for agroecology. A review. Agronomy for Sustainable Development, 32, 365-399. https://doi.org/10.1007/s13593-011-0036-y

Garnier, E., Stahl, U., Laporte, M.-A., Kattge, J., Mougenot, I., Kühn, I., ... Klotz, S. (2017). Towards a thesaurus of plant characteristics: An ecological contribution. Journal of Ecology, 105(2), 298-309. https://doi. org/10.1111/1365-2745.12698

Green, W. (2009). USDA PLANTS Compilation, version 1, 09-02-02. (http:// bricol.net/downloads/data/PLANTSdatabase/) NRCS: The PLANTS Database (http://plants.usda.gov, 1 Feb 2009). Baton Rouge, LA: National Plant Data Center.

Grime, J. P. (1974). Vegetation classification by reference to strategies. Nature, 250, 26-31. https://doi.org/10.1038/250026a0

Grime, J. P. (2001). Plant strategies, vegetation processes, and ecosystem properties. Chichester, UK: John Wiley \& Sons.

Grime, J. P. (2006). Trait convergence and trait divergence in herbaceous plant communities: Mechanisms and consequences. Journal of Vegetation Science, 17(2), 255-260. https://doi.org/10.1111/ j.1654-1103.2006.tb02444.x

Iversen, C. M., McCormack, M. L., Powell, A. S., Blackwood, C. B., Freschet, G. T., Kattge, J., ... Violle, C. (2017). A global Fine-Root Ecology Database to address below-ground challenges in plant ecology. New Phytologist, 215(1), 15-26. https://doi.org/10.1111/nph.14486

Jetz, W., Cavender-Bares, J., Pavlick, R., Schimel, D., Davis, F. W., Asner, G. P., ... Ustin, S. L. (2016). Monitoring plant functional diversity from space. Nature Plants, 2(3). https://doi.org/10.1038/nplants.2016.24

Kattge, J., Díaz, S., Lavorel, S., Prentice, I. C., Leadley, P., Bönisch, G., ... Wirth, C. (2011). TRY - A global database of plant traits. Global Change Biology, 17(9), 2905-2935. https://doi.org/10.1111/j.1365-2486.2011. 02451.x

Kattge, J., Diaz, S., \& Wirth, C. (2014). Of carrots and sticks: Commentary. Nature Geoscience, 7(11), 778-779. https://doi.org/10.1038/ngeo2280

Kattge, J., Ogle, K., Boenisch, G., Diaz, S., Lavorel, S., Madin, J., ... Wirth, C. (2011). A generic structure for plant trait databases. Methods in Ecology and Evolution, 2(2), 202-213. https://doi. org/10.1111/j.2041-210X.2010.00067.x

Kier, G., Mutke, J., Dinerstein, E., Ricketts, T. H., Küper, W., Kreft, H., \& Barthlott, W. (2005). Global patterns of plant diversity and floristic knowledge. Journal of Biogeography, 32(7), 1107-1116. https://doi. org/10.1111/j.1365-2699.2005.01272.x

Kissling, W. D., Walls, R., Bowser, A., Jones, M. O., Kattge, J., Agosti, D., ... Guralnick, R. P. (2018). Towards global data products of Essential Biodiversity Variables on species traits. Nature Ecology \& Evolution, 2(10), 1531-1540. https://doi.org/10.1038/s41559-018-0667-3

Kleyer, M., Bekker, R. M., Knevel, I. C., Bakker, J. P., Thompson, K., Sonnenschein, M., ... Peco, B. (2008). The LEDA Traitbase: A database of life-history traits of the Northwest European flora. Journal of Ecology, 96(6), 1266-1274. https://doi.org/10.1111/j.1365-2745.2008.01430.x 
Klotz, S., Kühn, I., \& Durka, W. (2002). BIOLFLOR - Eine Datenbank zu biologisch-ökologischen Merkmalen der Gefäßpflanzen in Deutschland - Schriftenreihe für Vegetationskunde 38. Bonn, Germany: Bundesamt Für Naturschutz.

König, C., Weigelt, P., Schrader, J., Taylor, A., Kattge, J., \& Kreft, H. (2019). Biodiversity data integration - The significance of data resolution and domain. PLoS Biology, 17(3), e3000183. https://doi.org/10.1371/ journal.pbio.3000183

Lavorel, S., Colloff, M. J., McIntyre, S., Doherty, M. D., Murphy, H. T., Metcalfe, D. J., ... Williams, K. J. (2015). Ecological mechanisms underpinning climate adaptation services. Global Change Biology, 21(1), 12-31. https://doi.org/10.1111/gcb.12689

Lavorel, S., \& Garnier, E. (2002). Predicting changes in community composition and ecosystem functioning from plant traits: Revisiting the Holy Grail. Functional Ecology, 16(5), 545-556. https://doi. org/10.1046/j.1365-2435.2002.00664.x

Lavorel, S., Storkey, J., Bardgett, R. D., de Bello, F., Berg, M. P., Le Roux, X., ... Harrington, R. (2013). A novel framework for linking functional diversity of plants with other trophic levels for the quantification of ecosystem services. Journal of Vegetation Science, 24(5), 942-948. https://doi.org/10.1111/jvs.12083

Loranger, J., Meyer, S. T., Shipley, B., Kattge, J., Loranger, H., Roscher, C., \& Weisser, W. W. (2012). Predicting invertebrate herbivory from plant traits: Evidence from 51 grassland species in experimental monocultures. Ecology, 93(12), 2674-2682. https://doi.org/10.1890/12-0328.1

Loranger, J., Meyer, S. T., Shipley, B., Kattge, J., Loranger, H., Roscher, C., ... Weisser, W. W. (2013). Predicting invertebrate herbivory from plant traits: Polycultures show strong nonadditive effects. Ecology, 94(7), 1499-1509. https://doi.org/10.1890/12-2063.1

Madin, J., Bowers, S., Schildhauer, M., Krivov, S., Pennington, D., \& Villa, F. (2007). An ontology for describing and synthesizing ecological observation data. Ecological Informatics, 2, 279-296. https://doi. org/10.1016/j.ecoinf.2007.05.004

Martin, A. R., Hale, C. E., Cerabolini, B. E. L., Cornelissen, J. H. C., Craine, J., Gough, W. A., ... Tirona, C. K. F. (2018). Inter- and intraspecific variation in leaf economic traits in wheat and maize. AoB PLANTS, 10(1), https://doi.org/10.1093/aobpla/ply006

Martin, A. R., \& Isaac, M. E. (2015). REVIEW: Plant functional traits in agroecosystems: A blueprint for research. Journal of Applied Ecology, 52(6), 1425-1435. https://doi.org/10.1111/1365-2664.12526

McGill, B. J., Enquist, B. J., Weiher, E., \& Westoby, M. (2006). Rebuilding community ecology from functional traits. Trends in Ecology \& Evolution, 21(4), 178-185. https://doi.org/10.1016/j.tree.2006.02.002

Moreno-Martínez, Á., Camps-Valls, G., Kattge, J., Robinson, N., Reichstein, M., van Bodegom, P., ... Running, S. W. (2018). A methodology to derive global maps of leaf traits using remote sensing and climate data. Remote Sensing of Environment, 218, 69-88. https://doi. org/10.1016/j.rse.2018.09.006

Newbold, T., Hudson, L. N., Hill, S. L. L., Contu, S., Lysenko, I., Senior, R. A., ... Purvis, A. (2015). Global effects of land use on local terrestrial biodiversity. Nature, 520(7545), 45-50. https://doi.org/10.1038/ nature14324

Olson, D. M., Dinerstein, E., Wikramanayake, E. D., Burgess, N. D., Powell, G. V. N., Underwood, E. C., ... Kassem, K. R. (2001). Terrestrial Ecoregions of the world: A new map of life on earth. BioScience, 51(11), 933. https://doi.org/10.1641/0006-3568(2001)051[0933:teotwa]2.0.co;2

Paula, S., Arianoutsou, M., Kazanis, D., Tavsanoglu, Ç., Lloret, F., Buhk, C., ... Pausas, J. G. (2009). Fire-related traits for plant species of the Mediterranean Basin. Ecology, 90(5), 1420. https://doi. org/10.1890/08-1309.1

Peaucelle, M., Bellassen, V., Ciais, P., Peñuelas, J., \& Viovy, N. (2016). A new approach to optimal discretization of plant functional types in a process-based ecosystem model with forest management: A case study for temperate conifers. Global Ecology and Biogeography, 26(4), 486-499. https://doi.org/10.1111/geb.12557
Pereira, H. M., Ferrier, S., Walters, M., Geller, G. N., Jongman, R. H. G., Scholes, R. J., ... Wegmann, M. (2013). Essential biodiversity variables. Science, 339(6117), 277-278. https://doi.org/10.1126/scien ce.1229931

Pérez-Harguindeguy, N., Díaz, S., Garnier, E., Lavorel, S., Poorter, H., Jaureguiberry, P., ... Cornelissen, J. H. C. (2013). New handbook for standardised measurement of plant functional traits worldwide. Australian Journal of Botany, 61(3), 167-234. https://doi.org/10.1071/ bt12225

Poschlod, P., Kleyer, M., Jackel, A.-K., Dannemann, A., \& Tackenberg, O. (2003). BIOPOP - A database of plant traits and internet application for nature conservation. Folia Geobotanica, 38(3), 263-271. https://doi.org/10.1007/bf02803198

Reichman, O. J., Jones, M. B., \& Schildhauer, M. P. (2011). Challenges and opportunities of open data in ecology. Science, 331(6018), 703-705. https://doi.org/10.1126/science.1197962

Royal Botanical Gardens KEW. (2008). Seed Information Database (SID). Version 7.1. Retrieved from http://data.kew.org/sid/

Sakschewski, B., von Bloh, W., Boit, A., Poorter, L., Peña-Claros, M., Heinke, J., ... Thonicke, K. (2016). Resilience of Amazon forests emerges from plant trait diversity. Nature Climate Change, 6(11), 1032-1036. https://doi.org/10.1038/nclimate3109

Sakschewski, B., vonBloh, W., Boit, A., Rammig, A., Kattge, J., Poorter, L., ... Thonicke, K. (2015). Leaf and stem economics spectra drive diversity of functional plant traits in a dynamic global vegetation model. Global Change Biology, 21(7), 2711-2725. https://doi.org/10.1111/gcb.12870

Schrodt, F., Kattge, J., Shan, H., Fazayeli, F., Joswig, J., Banerjee, A., ... Reich, P. B. (2015). BHPMF - A hierarchical Bayesian approach to gap-filling and trait prediction for macroecology and functional biogeography. Global Ecology and Biogeography, 24(12), 1510-1521. https://doi.org/10.1111/geb.12335

Shan, H., Kattge, J., Reich, P., Banerjee, A., Schrodt, F., \& Reichstein, M. (2012). Gap filling in the plant kingdom - Trait prediction using hierarchical probabilistic matrix factorization. Paper presented at the International Conference on Machine Learning (ICML), Edinburgh.

Smith, S. A., \& Brown, J. W. (2018). Constructing a broadly inclusive seed plant phylogeny. American Journal of Botany, 105(3), 302-314. https://doi.org/10.1002/ajb2.1019

ter Steege, H., Pitman, N. C. A., Sabatier, D., Baraloto, C., Salomão, R. P., Guevara, J. E., ... Silman, M. R. (2013). Hyperdominance in the Amazonian tree flora. Science, 342(6156). https://doi.org/10.1126/ science.1243092

Valladares, F., Gianoli, E., \& Gomez, J. M. (2007). Ecological limits to plant phenotypic plasticity. New Phytologist, 176, 749-763. https://doi. org/10.1111/j.1469-8137.2007.02275.x

Verheijen, L. M., Aerts, R., Brovkin, V., Cavender-Bares, J., Cornelissen, J. H. C., Kattge, J., \& van Bodegom, P. M. (2015). Inclusion of ecologically based trait variation in plant functional types reduces the projected land carbon sink in an earth system model. Global Change Biology, 21(8), 3074-3086. https://doi.org/10.1111/gcb.12871

Verheijen, L. M., Brovkin, V., Aerts, R., Bönisch, G., Cornelissen, J. H. C., Kattge, J., ... van Bodegom, P. M. (2013). Impacts of trait variation through observed trait-climate relationships on performance of an Earth system model: A conceptual analysis. Biogeosciences, 10(8), 5497-5515. https://doi.org/10.5194/bg-10-5497-2013

Violle, C., Navas, M. L., Vile, D., Kazakou, E., Fortunel, C., Hummel, I., \& Garnier, E. (2007). Let the concept of trait be functional!Oikos, 116(5), 882-892. https://doi.org/10.1111/j.2007.0030-1299.15559.x

von Humboldt, A.. (1817) De distributione geographica plantarum secundum coeli temperiem et altitudinem montium: Prolegomena. Lutetiae Parisiorum. In Libraria Graeco-Latino-Germanica. https://doi. org/10.5962/bhl.title.118581

Waltman, L., vanEck, N. J., \& Noyons, E. C. M. (2010). A unified approach to mapping and clustering of bibliometric networks. Journal of Informetrics, 4(4), 629-635. https://doi.org/10.1016/j.joi.2010.07.002 
Weigelt, P., König, C., \& Kreft, H. (2019). GIFT - A global inventory of floras and traits for macroecology and biogeography. Journal of Biogeography. https://doi.org/10.1111/jbi.13623

Westoby, M., \& Wright, I. J. (2006). Land-plant ecology on the basis of functional traits. Trends in Ecology \& Evolution, 21(5), 261-268. https://doi.org/10.1016/j.tree.2006.02.004

Wright, I. J., Dong, N., Maire, V., Prentice, I. C., Westoby, M., Díaz, S., ... Wilf, P. (2017). Global climatic drivers of leaf size. Science, 357(6354), 917-921. https://doi.org/10.1126/science.aal4760

Wright, I. J., Reich, P. B., Westoby, M., Ackerly, D. D., Baruch, Z., Bongers, F., ... Villar, R. (2004). The worldwide leaf economics spectrum. Nature, 428(6985), 821-827. https://doi.org/10.1038/nature02403

Xu, C. Y., \& Griffin, K. L. (2006). Seasonal variation in the temperature response of leaf respiration in Quercus rubra: Foliage respiration and leaf properties. Functional Ecology, 20(5), 778-789. https://doi. org/10.1111/j.1365-2435.2006.01161.x

Xu, L. K., \& Baldocchi, D. D. (2003). Seasonal trends in photosynthetic parameters and stomatal conductance of blue oak (Quercus douglasii) under prolonged summer drought and high temperature. Tree Physiology, 23(13), 865-877. https://doi.org/10.1093/treephys/ 23.13.865

Zanne, A. E., Tank, D. C., Cornwell, W. K., Eastman, J. M., Smith, S. A., FitzJohn, R. G., ... Beaulieu, J. M. (2014). Three keys to the radiation of angiosperms into freezing environments. Nature, 506(7486), 89-92. https://doi.org/10.1038/nature12872

\section{DATASET REFERENCES}

Abakumova, M., Zobel, K., Lepik, A., \& Semchenko, M. (2016). Plasticity in plant functional traits is shaped by variability in neighbourhood species composition. New Phytologist, 211(2), 455-463. https://doi. org/10.1111/nph.13935

Abedi, M., Bartelheimer, M., \& Poschlod, P. (2012). Aluminium toxic effects on seedling root survival affect plant composition along soil reaction gradients - A case study in dry sandy grasslands. Journal of Vegetation Science, 24(6), 1074-1085. https://doi.org/10.1111/ jvs.12016

Adamidis, G. C., Kazakou, E., Fyllas, N. M., \& Dimitrakopoulos, P. G. (2014). Species adaptive strategies and leaf economic relationships across serpentine and non-serpentine habitats on Lesbos, Eastern Mediterranean. PLoS ONE, 9(5), e96034. https://doi.org/10.1371/ journal.pone.0096034

Adler, P. B., Milchunas, D. G., Lauenroth, W. K., Sala, O. E., \& Burke, I. C. (2004). Functional traits of graminoids in semi-arid steppes: A test of grazing histories. Journal of Applied Ecology, 41(4), 653-663. https://doi.org/10.1111/j.0021-8901.2004.00934.x

Adler, P. B., Salguero-Gomez, R., Compagnoni, A., Hsu, J. S., RayMukherjee, J., Mbeau-Ache, C., \& Franco, M. (2013). Functional traits explain variation in plant life history strategies. Proceedings of the National Academy of Sciences of the United States of America, 111(2), 740-745. https://doi.org/10.1073/pnas.1315179111

Adriaenssens, S. (2012). Dry deposition and canopy exchange for temperate tree species under high nitrogen deposition. (PhD), Ghent University, Ghent, Belgium.

Albert, C. H., de Bello, F., Boulangeat, I., Pellet, G., Lavorel, S., \& Thuiller, W. (2011). On the importance of intraspecific variability for the quantification of functional diversity. Oikos, 121(1), 116-126. https://doi. org/10.1111/j.1600-0706.2011.19672.x

Albert, C. H., Thuiller, W., Yoccoz, N. G., Soudant, A., Boucher, F., Saccone, P., \& Lavorel, S. (2010). Intraspecific functional variability: Extent, structure and sources of variation. Journal of Ecology, 98(3), 604-613. https://doi.org/10.1111/j.1365-2745.2010.01651.x

Aleixo, I., Norris, D., Hemerik, L., Barbosa, A., Prata, E., Costa, F., \& Poorter, L. (2019). Amazonian rainforest tree mortality driven by climate and functional traits. Nature Climate Change, 9(5), 384-388. https://doi.org/10.1038/s41558-019-0458-0

Ali, H. E., Reineking, B., \& Münkemüller, T. (2017). Effects of plant functional traits on soil stability: Intraspecific variability matters. Plant and Soil, 411(1-2), 359-375. https://doi.org/10.1007/s11104-016-3036-5

Almeida, D., Domingues, T. F., Ehleringer, J., Martinelli, L. A., Cook, C., Flanagan, L., \& Ometto, J. P. (2001). LBA-ECO CD-02 Leaf Water Potential, Forest and Pasture Sites, Para, Brazil: 2000-2001. Retrieved from http://daac.ornl.gov/cgi-bin/dsviewer.pl?ds_xml:id=1100

Apgaua, D. M. G., Ishida, F. Y., Tng, D. Y. P., Laidlaw, M. J., Santos, R. M., Rumman, R., ... Laurance, S. G. W. (2015). Functional traits and water transport strategies in lowland tropical rainforest trees. PLOS ONE, 10(6), e0130799. https://doi.org/10.1371/journal.pone.0130799

Atkin, O. K., Bloomfield, K. J., Reich, P. B., Tjoelker, M. G., Asner, G. P., Bonal, D., ... Zaragoza-Castells, J. (2015). Global variability in leaf respiration in relation to climate, plant functional types and leaf traits. New Phytologist, 206(2), 614-636. https://doi.org/10.1111/ nph.13253

Aubin, I., Messier, C., Gachet, S., Lawrence, K., McKenney, D., Arseneault, A., ... Munson, A. D. (2012). TOPIC-traits of plants in Canada. Retrieved from http://cfs.cloud.nrcan.gc.ca/ctn/topic.php

Auger, S., \& Shipley, B. (2012). Inter-specific and intra-specific trait variation along short environmental gradients in an old-growth temperate forest. Journal of Vegetation Science, 24(3), 419-428. https://doi. org/10.1111/j.1654-1103.2012.01473.x

Baastrup-Spohr, L., Sand-Jensen, K., Nicolajsen, S. V., \& Bruun, H. H. (2015). From soaking wet to bone dry: Predicting plant community composition along a steep hydrological gradient. Journal of Vegetation Science, 26(4), 619-630. https://doi.org/10.1111/jvs.12280

Bahar, N. H. A., Ishida, F. Y., Weerasinghe, L. K., Guerrieri, R., O'Sullivan, O. S., Bloomfield, K. J., ... Atkin, O. K. (2016). Leaf-level photosynthetic capacity in lowland Amazonian and high-elevation Andean tropical moist forests of Peru. New Phytologist, 214(3), 1002-1018. https://doi.org/10.1111/nph.14079

Bahn, M., Wohlfahrt, G., Haubner, E., Horak, I., Michaeler, W., Rottmar, K., ... Cernusca, A. (1999). Leaf photosynthesis, nitrogen contents and specific leaf area of 30 grassland species in differently managed mountain ecosystems in the Eastern Alps. In A. Cernusca, U. Tappeiner, \& N. Bayfield (Eds.), Land-use changes in European mountain ecosystems. ECOMONT- Concept and Results (pp. 247-255). Berlin, Germany: Blackwell Wissenschaft.

Baraloto, C., Timothy Paine, C. E., Poorter, L., Beauchene, J., Bonal, D., Domenach, A.-M., ... Chave, J. (2010). Decoupled leaf and stem economics in rain forest trees. Ecology Letters, 13(11), 1338-1347. https://doi.org/10.1111/j.1461-0248.2010.01517.x

Baruch, Z., \& Goldstein, G. (1999). Leaf construction cost, nutrient concentration, and net $\mathrm{CO}_{2}$ assimilation of native and invasive species in Hawaii. Oecologia, 121(2), 183-192. https://doi.org/10.1007/s0044 20050920

Bauerle, W. L., Oren, R., Way, D. A., Qian, S. S., Stoy, P. C., Thornton, P. E., ... Reynolds, R. F. (2012). Photoperiodic regulation of the seasonal pattern of photosynthetic capacity and the implications for carbon cycling. Proceedings of the National Academy of Sciences of the United States of America, 109(22), 8612-8617. https://doi.org/10.1073/ pnas.1119131109

Bauters, M., Ampoorter, E., Huygens, D., Kearsley, E., De Haulleville, T., Sellan, G., ... Verheyen, K. (2015). Functional identity explains carbon sequestration in a 77-year-old experimental tropical plantation. Ecosphere, 6(10), art198. https://doi.org/10.1890/es15-00342.1

Bauters, M., Verbeeck, H., Demol, M., Bruneel, S., Taveirne, C., Van der Heyden, D., ... Boeckx, P. (2017). Parallel functional and stoichiometric trait shifts in South American and African forest communities with elevation. Biogeosciences, 14(23), 5313-5321. https://doi. org/10.5194/bg-14-5313-2017 
Bauters, M., Vercleyen, O., Vanlauwe, B., Six, J., Bonyoma, B., Badjoko, H., ... Boeckx, P. (2019). Long-term recovery of the functional community assembly and carbon pools in an African tropical forest succession. Biotropica, 51(3), 319-329. https://doi.org/10.1111/btp. 12647

Beckmann, M., Hock, M., Bruelheide, H., \& Erfmeier, A. (2012). The role of UV-B radiation in the invasion of Hieracium pilosella-A comparison of German and New Zealand plants. Environmental and Experimental Botany, 75, 173-180. https://doi.org/10.1016/j.envex pbot.2011.09.010

Belluau, M., \& Shipley, B. (2017). Predicting habitat affinities of herbaceous dicots to soil wetness based on physiological traits of drought tolerance. Annals of Botany, 119(6), 1073-1084. https://doi. org/10.1093/aob/mcw267

Belluau, M., \& Shipley, B. (2018). Linking hard and soft traits: Physiology, morphology and anatomy interact to determine habitat affinities to soil water availability in herbaceous dicots. PLOS ONE, 13(3), e0193130. https://doi.org/10.1371/journal.pone.0193130

Benomar, L., Lamhamedi, M. S., Pepin, S., Rainville, A., Lambert, M.-C., Margolis, H. A., ... Beaulieu, J. (2018). Thermal acclimation of photosynthesis and respiration of southern and northern white spruce seed sources tested along a regional climatic gradient indicates limited potential to cope with temperature warming. Annals of Botany, 121(3), 443-457. https://doi.org/10.1093/aob/mcx174

Bergmann, J., Ryo, M., Prati, D., Hempel, S., \& Rillig, M. C. (2017). Root traits are more than analogues of leaf traits: The case for diaspore mass. New Phytologist, 216(4), 1130-1139. https://doi.org/10.1111/ nph.14748

Berner, L. T., Alexander, H. D., Loranty, M. M., Ganzlin, P., Mack, M. C., Davydov, S. P., \& Goetz, S. J. (2015). Biomass allometry for alder, dwarf birch, and willow in boreal forest and tundra ecosystems of far northeastern Siberia and north-central Alaska. Forest Ecology and Management, 337, 110-118. https://doi.org/10.1016/j.foreco.2014.10.027

Bernhardt-Römermann, M., Poschlod, P., \& Hentschel, J. (2018). BryForTrait - A life-history trait database of forest bryophytes. Journal of Vegetation Science, 29(4), 798-800. https://doi.org/10.1111/ jvs.12646

Blonder, B., Baldwin, B. G., Enquist, B. J., \& Robichaux, R. H. (2015). Variation and macroevolution in leaf functional traits in the Hawaiian silversword alliance (Asteraceae). Journal of Ecology, 104(1), 219-228. https://doi.org/10.1111/1365-2745.12497

Blonder, B., \& Enquist, B. J. (2014). Inferring climate from angiosperm leaf venation networks. New Phytologist, 204(1), 116-126. https://doi. org/10.1111/nph.12780

Blonder, B., Kapas, R. E., Dalton, R. M., Graae, B. J., Heiling, J. M., \& Opedal, $\varnothing$. H. (2018). Microenvironment and functional-trait context dependence predict alpine plant community dynamics. Journal of Ecology, 106(4), 1323-1337. https://doi.org/10.1111/1365-2745.12973

Blonder, B., Royer, D. L., Johnson, K. R., Miller, I., \& Enquist, B. J. (2014). Plant ecological strategies shift across the cretaceous-paleogene boundary. PLoS Biology, 12(9), e1001949. https://doi.org/10.1371/ journal.pbio.1001949

Blonder, B., Vasseur, F., Violle, C., Shipley, B., Enquist, B. J., \& Vile, D. (2015). Testing models for the leaf economics spectrum with leaf and whole-plant traits in Arabidopsis thaliana. AoB PLANTS, 7, plv049. https://doi.org/10.1093/aobpla/plv049

Blonder, B., Violle, C., Bentley, L. P., \& Enquist, B. J. (2010). Venation networks and the origin of the leaf economics spectrum. Ecology Letters, 14(2), 91-100. https://doi.org/10.1111/j.1461-0248.2010.01554.x

Blonder, B., Violle, C., \& Enquist, B. J. (2013). Assessing the causes and scales of the leaf economics spectrum using venation networks in Populus tremuloides. Journal of Ecology, 101(4), 981-989. https://doi. org/10.1111/1365-2745.12102

Bocanegra-González, K. T., Fernández-Méndez, F., \& Galvis-Jiménez, J. D. (2015). Grupos funcionales de árboles en bosques secundarios de la región Bajo Calima (Buenaventura, Colombia). Boletín Científico Del Centro De Museos, 19(1), 17-40. https://doi.org/10.17151/ bccm.2015.19.1.2

Bond-Lamberty, B., Gower, S. T., Wang, C., Cyr, P., \& Veldhuis, H. (2006). Nitrogen dynamics of a boreal black spruce wildfire chronosequence. Biogeochemistry, 81(1), 1-16. https://doi.org/10.1007/ s10533-006-9025-7

Bond-Lamberty, B., Wang, C., Gower, S. T., \& Norman, J. (2002). Leaf area dynamics of a boreal black spruce fire chronosequence. Tree Physiology, 22(14), 993-1001. https://doi.org/10.1093/treep hys/22.14.993

Boucher, F. C., Thuiller, W., Arnoldi, C., Albert, C. H., \& Lavergne, S. (2013). Unravelling the architecture of functional variability in wild populations of Polygonum viviparum L. Functional Ecology, 27(2), 382391. https://doi.org/10.1111/1365-2435.12034

Boukili, V. K., \& Chazdon, R. L. (2017). Environmental filtering, local site factors and landscape context drive changes in functional trait composition during tropical forest succession. Perspectives in Plant Ecology, Evolution and Systematics, 24, 37-47. https://doi. org/10.1016/j.ppees.2016.11.003

Brown, K. A., Johnson, S. E., Parks, K. E., Holmes, S. M., Ivoandry, T., Abram, N. K., ... Wright, P. C. (2013). Use of provisioning ecosystem services drives loss of functional traits across land use intensification gradients in tropical forests in Madagascar. Biological Conservation, 161, 118-127. https://doi.org/10.1016/j.biocon.2013.03.014

Brumnich, F., Marchetti, Z. Y., \& Pereira, M. S. (2019). Changes in forest diversity over a chronosequence of fluvial islands. iForest Biogeosciences and Forestry, 12(3), 306-316. https://doi.org/10.3832/ ifor2737-012

Bruun, H. H. (2019). Dataset on reproductive traits of Scandinavian alpine plants. Data in Brief, 25. https://doi.org/10.1016/j.dib.2019.104149

Bruy, D., Hattermann, T., Barrabé, L., Mouly, A., Barthélémy, D., \& Isnard, S. (2018). Evolution of plant architecture, functional diversification and divergent evolution in the genus Atractocarpus (Rubiaceae) for New Caledonia. Frontiers in Plant Science, 9. https://doi.org/10.3389/ fpls.2018.01775

Buchanan, S., Isaac, M. E., Van den Meersche, K., \& Martin, A. R. (2018). Functional traits of coffee along a shade and fertility gradient in coffee agroforestry systems. Agroforestry Systems, 93(4), 1261-1273. https://doi.org/10.1007/s10457-018-0239-1

Bucher, S. F., Auerswald, K., Tautenhahn, S., Geiger, A., Otto, J., Müller, A., \& Römermann, C. (2016). Inter- and intraspecific variation in stomatal pore area index along elevational gradients and its relation to leaf functional traits. Plant Ecology, 217(3), 229-240. https://doi. org/10.1007/s11258-016-0564-2

Burrascano, S., Copiz, R., Del Vico, E., Fagiani, S., Giarrizzo, E., Mei, M., ... Blasi, C. (2015). Wild boar rooting intensity determines shifts in understorey composition and functional traits. Community Ecology, 16(2), 244-253. https://doi.org/10.1556/168.2015.16.2.12

Butterfield, B. J., \& Briggs, J. M. (2010). Regeneration niche differentiates functional strategies of desert woody plant species. Oecologia, 165(2), 477-487. https://doi.org/10.1007/s00442-010-1741-y

Byun, C., deBlois, S., \& Brisson, J. (2012). Plant functional group identity and diversity determine biotic resistance to invasion by an exotic grass. Journal of Ecology, 101(1), 128-139. https://doi. org/10.1111/1365-2745.12016

Cadotte, M. W. (2017). Functional traits explain ecosystem function through opposing mechanisms. Ecology Letters, 20(8), 989-996. https://doi.org/10.1111/ele.12796

Cailleret, M., Jansen, S., Robert, E. M. R., Desoto, L., Aakala, T., Antos, J. A., ... Martinez-Vilalta, J. (2017). A synthesis of radial growth patterns preceding tree mortality. Global Change Biology, 23(4), 1675-1690. https://doi.org/10.1111/gcb.13535

Campany, C. E., Martin, L., \& Watkins, J. E. (2018). Convergence of ecophysiological traits drives floristic composition of early lineage 
vascular plants in a tropical forest floor. Annals of Botany, 123(5), 793-803. https://doi.org/10.1093/aob/mcy210

Campbell, C., Atkinson, L., Zaragoza-Castells, J., Lundmark, M., Atkin, O., \& Hurry, V. (2007). Acclimation of photosynthesis and respiration is asynchronous in response to changes in temperature regardless of plant functional group. New Phytologist, 176(2), 375-389. https://doi. org/10.1111/j.1469-8137.2007.02183.x

Campetella, G., Botta-Dukát, Z., Wellstein, C., Canullo, R., Gatto, S., Chelli, S., ... Bartha, S. (2011). Patterns of plant trait-environment relationships along a forest succession chronosequence. Agriculture, Ecosystems \& Environment, 145(1), 38-48. https://doi.org/10.1016/ j.agee.2011.06.025

Campetella, G., Chelli, S., Wellstein, C., Farris, E., Calvia, G., Simonetti, E., ... Marignani, M. (2019). Contrasting patterns in leaf traits of Mediterranean shrub communities along an elevation gradient: Measurements matter. Plant Ecology, 220(7-8), 765-776. https://doi. org/10.1007/s11258-019-00951-y

Carvalho, F., Brown, K. A., Waller, M. P., \& Boom, A. (2019). Leaf traits interact with management and water table to modulate ecosystem properties in fen peatlands. Plant and Soil, 441(1-2), 331-347. https://doi.org/10.1007/s11104-019-04126-6

Carvalho, F., Brown, K. A., Waller, M. P., Bunting, M. J., Boom, A., \& Leng, M. J. (2019). A method for reconstructing temporal changes in vegetation functional trait composition using Holocene pollen assemblages. PLoS ONE, 14(5), e0216698. https://doi.org/10.1371/journ al.pone.0216698

Castro-Díez, P., Puyravaud, J. P., Cornelissen, J. H. C., \& Villar-Salvador, P. (1998). Stem anatomy and relative growth rate in seedlings of a wide range of woody plant species and types. Oecologia, 116(1-2), 57-66. https://doi.org/10.1007/s004420050563

Catford, J. A., Morris, W. K., Vesk, P. A., Gippel, C. J., \& Downes, B. J. (2014). Species and environmental characteristics point to flow regulation and drought as drivers of riparian plant invasion. Diversity and Distributions, 20(9), 1084-1096. https://doi.org/10.1111/ddi.12225

Catford, J. A., Smith, A. L., Wragg, P. D., Clark, A. T., Kosmala, M., Cavender-Bares, J., ... Tilman, D. (2019). Traits linked with species invasiveness and community invasibility vary with time, stage and indicator of invasion in a long-term grassland experiment. Ecology Letters, 22(4), 593-604. https://doi.org/10.1111/ele.13220

Cavender-Bares, J., Keen, A., \& Miles, B. (2006). Phylogenetic structure of Floridian plant communities depends on taxonomic and spatial scale. Ecology, 87(sp7), S109-S122. https://doi. org/10.1890/0012-9658(2006)87[109:psofpc]2.0.co;2

Cerabolini, B. E. L., Brusa, G., Ceriani, R. M., De Andreis, R., Luzzaro, A., \& Pierce, S. (2010). Can CSR classification be generally applied outside Britain?Plant Ecology, 210(2), 253-261. https://doi.org/10.1007/ s11258-010-9753-6

Cerabolini, B. E. L., Pierce, S., Luzzaro, A., \& Ossola, A. (2009). Species evenness affects ecosystem processes in situ via diversity in the adaptive strategies of dominant species. Plant Ecology, 207(2), 333345. https://doi.org/10.1007/s11258-009-9677-1

Chacón-Madrigal, E., Wanek, W., Hietz, P., \& Dullinger, S. (2018). Traits indicating a conservative resource strategy are weakly related to narrow range size in a group of neotropical trees. Perspectives in Plant Ecology, Evolution and Systematics, 32, 30-37. https://doi. org/10.1016/j.ppees.2018.01.003

Chain-Guadarrama, A., Imbach, P., Vilchez-Mendoza, S., Vierling, L., \& Finegan, B. (2017). Potential trajectories of old-growth Neotropical forest functional composition under climate change. Ecography, 41, 75-89.

Chambers, J. Q., Tribuzy, E. S., Toledo, L. C., Crispim, B. F., Higuchi, N., dosSantos, J., ... Trumbore, S. E. (2004). Respiration from a tropical forest ecosystem: Partitioning of sources and low carbon use efficiency. Ecological Applications, 14(sp4), 72-88. https://doi.org/ 10.1890/01-6012
Chave, J., Coomes, D., Jansen, S., Lewis, S. L., Swenson, N. G., \& Zanne, A. E. (2009). Towards a worldwide wood economics spectrum. Ecology Letters, 12(4), 351-366. https://doi.org/10.1111/j.1461-0248. 2009.01285.x

Chen, A., Lichstein, J. W., Osnas, J. L. D., \& Pacala, S. W. (2014). Speciesindependent down-regulation of leaf photosynthesis and respiration in response to shading: Evidence from six temperate tree species. PLoS ONE, 9(4), e91798. https://doi.org/10.1371/journal.pone.0091798

Chen, S.-C., Cornwell, W. K., Zhang, H.-X., \& Moles, A. T. (2017). Plants show more flesh in the tropics: Variation in fruit type along latitudinal and climatic gradients. Ecography, 40(4), 531-538. https://doi. org/10.1111/ecog.02010

Chen, Y. H., Han, W. X., Tang, L. Y., Tang, Z. Y., \& Fang, J. Y. (2013). Leaf nitrogen and phosphorus concentrations of woody plants differ in responses to climate, soil and plant growth form. Ecography, 36(2), 178-184. https://doi.org/10.1111/j.1600-0587.2011.06833.x

Chianucci, F., Pisek, J., Raabe, K., Marchino, L., Ferrara, C., \& Corona, P. (2018). A dataset of leaf inclination angles for temperate and boreal broadleaf woody species. Annals of Forest Science, 75(2). https://doi. org/10.1007/s13595-018-0730-x

Choat, B., Jansen, S., Brodribb, T. J., Cochard, H., Delzon, S., Bhaskar, R., ... Zanne, A. E. (2012). Global convergence in the vulnerability of forests to drought. Nature, 491(7426), 752-755. https://doi. org/10.1038/nature11688

Chrobock, T., Kempel, A., Fischer, M., \& van Kleunen, M. (2011). Introduction bias: Cultivated alien plant species germinate faster and more abundantly than native species in Switzerland. Basic and Applied Ecology, 12(3), 244-250. https://doi.org/10.1016/j.baae.2011.03.001

Chung, K.-S., Hipp, A. L., \& Roalson, E. H. (2012). Chromosome number evolves independently of genome size in a clade with nonlocalized centromeres (Carex: Cyperaceae). Evolution, 66(9), 2708-2722. https://doi.org/10.1111/j.1558-5646.2012.01624.x

Chytrý, M., Tichý, L., Dřevojan, P., Sádlo, J., \& Zelený, D. (2018). Ellenbergtype indicator values for the Czech flora. Preslia, 90(2), 83-103. https://doi.org/10.23855/preslia.2018.083

Ciccarelli, D. (2015). Mediterranean coastal dune vegetation: Are disturbance and stress the key selective forces that drive the psammophilous succession?Estuarine, Coastal and Shelf Science, 165, 247-253. https://doi.org/10.1016/j.ecss.2015.05.023

Ciocârlan, V. (2009). The illustrated Flora of Romania. Pteridophyta et Spermatopyta (in Romanian). Editura Ceres. 1141 pp.

Cornelissen, J. H. C., Diez, P. C., \& Hunt, R. (1996). Seedling growth, allocation and leaf attributes in a wide range of woody plant species and types. The Journal of Ecology, 84(5), 755. https://doi. org/10.2307/2261337

Cornelissen, J. H. C., Quested, H. M., Gwynn-Jones, D., Van Logtestijn, R. S. P., De Beus, M. A. H., Kondratchuk, A., ... Aerts, R. (2004). Leaf digestibility and litter decomposability are related in a wide range of subarctic plant species and types. Functional Ecology, 18(6), 779-786. https://doi.org/10.1111/j.0269-8463.2004.00900.x

Cornwell, W. K., Cornelissen, J. H. C., Amatangelo, K., Dorrepaal, E., Eviner, V. T., Godoy, O., ... Westoby, M. (2008). Plant species traits are the predominant control on litter decomposition rates within biomes worldwide. Ecology Letters, 11(10), 1065-1071. https://doi. org/10.1111/j.1461-0248.2008.01219.x

Cornwell, W. K., Wright, I. J., Turner, J., Maire, V., Barbour, M. M., Cernusak, L. A., ... Santiago, L. S. (2018). Climate and soils together regulate photosynthetic carbon isotope discrimination within $\mathrm{C} 3$ plants worldwide. Global Ecology and Biogeography, 27(9), 1056-1067. https://doi.org/10.1111/geb.12764

Craine, J. M., Elmore, A. J., Aidar, M. P. M., Bustamante, M., Dawson, T. E., Hobbie, E. A., ... Wright, I. J. (2009). Global patterns of foliar nitrogen isotopes and their relationships with climate, mycorrhizal fungi, foliar nutrient concentrations, and nitrogen availability. New Phytologist, 183(4), 980-992. https://doi.org/10.1111/j.1469-8137.2009.02917.x 
Craine, J. M., Lee, W. G., Bond, W. J., Williams, R. J., \& Johnson, L. C. (2005). Environmental constraints on a global relationship among leaf and root traits of grasses. Ecology, 86(1), 12-19. https://doi. org/10.1890/04-1075

Craine, J. M., Nippert, J. B., Towne, E. G., Tucker, S., Kembel, S. W., Skibbe, A., \& McLauchlan, K. K. (2011). Functional consequences of climate change-induced plant species loss in a tallgrass prairie. Oecologia, 165(4), 1109-1117. https://doi.org/10.1007/s00442-011-1938-8

Craven, D., Braden, D., Ashton, M. S., Berlyn, G. P., Wishnie, M., \& Dent, D. (2007). Between and within-site comparisons of structural and physiological characteristics and foliar nutrient content of 14 tree species at a wet, fertile site and a dry, infertile site in Panama. Forest Ecology and Management, 238(1-3), 335-346. https://doi. org/10.1016/j.foreco.2006.10.030

Dahlin, K. M., Asner, G. P., \& Field, C. B. (2013). Environmental and community controls on plant canopy chemistry in a Mediterraneantype ecosystem. Proceedings of the National Academy of Sciences of the United States of America, 110(17), 6895-6900. https://doi. org/10.1073/pnas.1215513110

Dainese, M., \& Bragazza, L. (2012). Plant traits across different habitats of the Italian Alps: A comparative analysis between native and alien species. Alpine Botany, 122, 11-21. https://doi.org/10.1007/ s00035-012-0101-4

Dalke, I. V., Novakovskiy, A. B., Maslova, S. P., \& Dubrovskiy, Y. A. (2018). Morphological and functional traits of herbaceous plants with different functional types in the European Northeast. Plant Ecology, 219(11), 1295-1305. https://doi.org/10.1007/s11258-018-0879-2

Dang-Le, A. T., Edelin, C., \& Le-Cong, K. (2013). Ontogenetic variations in leaf morphology of the tropical rain forest species Dipterocarpus alatus Roxb. ex G. Don. Trees, 27(3), 773-786. https://doi.org/10.1007/ s00468-012-0832-2

Dawson, S. K., Warton, D. I., Kingsford, R. T., Berney, P., Keith, D. A., \& Catford, J. A. (2017). Plant traits of propagule banks and standing vegetation reveal flooding alleviates impacts of agriculture on wetland restoration. Journal of Applied Ecology, 54(6), 1907-1918. https://doi.org/10.1111/1365-2664.12922

deAraujo, A. C., Ometto, J. P. H. B., Dolman, A. J., Kruijt, B., Waterloo, M. J., \& Ehleringer, J. R. (2012). LBA-ECO CD-02 C and N isotopes in leaves and atmospheric $\mathrm{CO}_{2}$, Amazonas, Brazil. Retrieved from http://dx.doi. org/10.3334/ORNLDAAC/1097

deFrutos, Á., Navarro, T., Pueyo, Y., \& Alados, C. L. (2015). Inferring resilience to fragmentation-induced changes in plant communities in a semi-arid Mediterranean ecosystem. PLoS ONE, 10(3), e0118837. https://doi.org/10.1371/journal.pone.0118837

De Long, J. R., Jackson, B. G., Wilkinson, A., Pritchard, W. J., Oakley, S., Mason, K. E., ... Bardgett, R. D. (2019). Relationships between plant traits, soil properties and carbon fluxes differ between monocultures and mixed communities in temperate grassland. Journal of Ecology, 107(4), 1704-1719. https://doi.org/10.1111/1365-2745.13160

deVries, F. T., \& Bardgett, R. D. (2016). Plant community controls on short-term ecosystem nitrogen retention. New Phytologist, 210(3), 861-874. https://doi.org/10.1111/nph.13832

Dechant, B., Cuntz, M., Vohland, M., Schulz, E., \& Doktor, D. (2017). Estimation of photosynthesis traits from leaf reflectance spectra: Correlation to nitrogen content as the dominant mechanism. Remote Sensing of Environment, 196, 279-292. https://doi.org/10.1016/j. rse.2017.05.019

Delpierre, N., Berveiller, D., Granda, E., \& Dufrêne, E. (2015). Wood phenology, not carbon input, controls the interannual variability of wood growth in a temperate oak forest. New Phytologist, 210(2), 459-470. https://doi.org/10.1111/nph.13771

Demey, A., Staelens, J., Baeten, L., Boeckx, P., Hermy, M., Kattge, J., \& Verheyen, K. (2013). Nutrient input from hemiparasitic litter favors plant species with a fast-growth strategy. Plant and Soil, 371(1-2), 53-66. https://doi.org/10.1007/s11104-013-1658-4
Domingues, T. F., Martinelli, L. A., \& Ehleringer, J. R. (2007). Ecophysiological traits of plant functional groups in forest and pasture ecosystems from eastern Amazônia, Brazil. Plant Ecology, 193(1), 101-112. https://doi.org/10.1007/s11258-006-9251-z

Domingues, T. F., Meir, P., Feldpausch, T. R., Saiz, G., Veenendaal, E. M., Schrodt, F., ... Lloyd, J. O. N. (2010). Co-limitation of photosynthetic capacity by nitrogen and phosphorus in West Africa woodlands. Plant, Cell \& Environment, 33(6), 959-980. https://doi. org/10.1111/j.1365-3040.2010.02119.x

Dong, N., Prentice, I. C., Evans, B. J., Caddy-Retalic, S., Lowe, A. J., \& Wright, I. J. (2017). Leaf nitrogen from first principles: Field evidence for adaptive variation with climate. Biogeosciences, 14(2), 481-495. https://doi.org/10.5194/bg-14-481-2017

Dressler, S., Schmidt, M., \& Zizka, G. (2014). Introducing 'African plants A Photo Guide' - An interactive photo data-base and rapid identification tool for continental Africa. Taxon, 63(5), 1159-1161. https://doi. org/10.12705/635.26

Dwyer, J. M., Hobbs, R. J., \& Mayfield, M. M. (2014). Specific leaf area responses to environmental gradients through space and time. Ecology, 95(2), 399-410. https://doi.org/10.1890/13-0412.1

Ellenberg, H., \& Leuschner, C. (2010). Vegetation Mitteleuropas mit den Alpen in ökologischer, dynamischer und historischer Sicht (6th ed.). Stuttgart, Germany: Ulmer.

Fagúndez, J., \& Izco, J. (2008). Seed morphology of two distinct species of Erica L. (Ericaceae). Acta Botanica Malacitana, 33, 1-9.

Fagúndez, J., Juan, R., Fernández, I., Pastor, J., \& Izco, J. (2010). Systematic relevance of seed coat anatomy in the European heathers (Ericeae, Ericaceae). Plant Systematics and Evolution, 284(1-2), 65-76. https://doi.org/10.1007/s00606-009-0240-2

Falster, D. S., Duursma, R. A., Ishihara, M. I., Barneche, D. R., FitzJohn, R. G., Vårhammar, A., ... York, R. A. (2015). BAAD: A biomass and allometry database for woody plants. Ecology, 96(5), 1445-1445. https://doi. org/10.1890/14-1889.1

Fazlioglu, F. (2008). Numerical analysis of Taeniatherum caput-medusae collected throughout Turkey. Report from project at Middle East Technical University (METU), Ankara, Turkey.

Fazlioglu, F. (2011). A phenetics for infrageneric grouping of Limonium Mill. genus (Plumbaginaceae) in Turkey. Master thesis, Middle East Technical University (METU), Ankara, Turkey.

Fazlioglu, F., Al-Namazi, A., \& Bonser, S. P. (2016). Reproductive efficiency and shade avoidance plasticity under simulated competition. Ecology and Evolution, 6(14), 4947-4957. https://doi.org/10.1002/ece3.2254

Fazlioglu, F., \& Bonser, S. P. (2016). Phenotypic plasticity and specialization in clonal versus non-clonal plants: A data synthesis. Acta Oecologica, 77, 193-200. https://doi.org/10.1016/j.actao.2016.10.012

Fazlioglu, F., Wan, J. S. H., \& Bonser, S. P. (2016). Testing specialization hypothesis on a stress gradient. Austral Ecology, 42(1), 40-47. https://doi.org/10.1111/aec.12399

Fazlioglu, F., Wan, J. S. H., \& Bonser, S. P. (2018). Phenotypic plasticity and specialization along an altitudinal gradient in Trifolium repens. Turkish Journal of Botany, 42(4), 440-447. https://doi.org/10.3906/ bot-1711-21

Feng, Y., \& van Kleunen, M. (2014). Responses to shading of naturalized and non-naturalized exotic woody species. Annals of Botany, 114(5), 981-989. https://doi.org/10.1093/aob/mcu163

Findurová, A. (2018). Variability of leaf traits SLA and LDMC in selected species of the Czech flora (Master thesis). Brno, Czech Republic: Masaryk University.

Finegan, B., Peña-Claros, M., de Oliveira, A., Ascarrunz, N., Bret-Harte, M. S., Carreño-Rocabado, G., ... Poorter, L. (2015). Does functional trait diversity predict above-ground biomass and productivity of tropical forests? Testing three alternative hypotheses. Journal of Ecology, 103, 191-201.

Firn, J., McGree, J. M., Harvey, E., Flores-Moreno, H., Schütz, M., Buckley, Y. M., ... Risch, A. C. (2019). Leaf nutrients, not specific leaf 
area, are consistent indicators of elevated nutrient inputs. Nature Ecology \& Evolution, 3(3), 400-406. https://doi.org/10.1038/ s41559-018-0790-1

Flowers, T. J., Santos, J., Jahns, M., Warburton, B., \& Reed, P. (2017). eHALOPH - Halophytes database (version 3.11) accessed 2017. Retrieved from http://www.sussex.ac.uk/affiliates/halophytes

Fonseca, C. R., Overton, J. M., Collins, B., \& Westoby, M. (2000). Shifts in trait-combinations along rainfall and phosphorus gradients. Journal of Ecology, 88(6), 964-977. https://doi.org/10.1046/ j.1365-2745.2000.00506.x

Forgiarini, C., Souza, A. F., Longhi, S. J., \& Oliveira, J. M. (2014). In the lack of extreme pioneers: Trait relationships and ecological strategies of 66 subtropical tree species. Journal of Plant Ecology, 8(4), 359-367. https://doi.org/10.1093/jpe/rtu028

Fortunel, C., McFadden, I. R., Valencia, R., \& Kraft, N. J. B. (2019). Neither species geographic range size, climatic envelope, nor intraspecific leaf trait variability capture habitat specialization in a hyperdiverse Amazonian forest. Biotropica, 51(3), 304-310. https://doi. org/10.1111/btp.12643

Frenette-Dussault, C., Shipley, B., Léger, J.-F., Meziane, D., \& Hingrat, Y. (2011). Functional structure of an arid steppe plant community reveals similarities with Grime's C-S-R theory. Journal of Vegetation Science, 23(2), 208-222. https://doi.org/10.1111/j.1654-1103.2011.01350.x

Freschet, G. T., Cornelissen, J. H. C., vanLogtestijn, R. S. P., \& Aerts, R. (2010). Evidence of the 'plant economics spectrum' in a subarctic flora. Journal of Ecology, 98(2), 362-373. https://doi.org/ 10.1111/j.1365-2745.2009.01615.x

Freschet, G. T., Kichenin, E., \& Wardle, D. A. (2015). Explaining within-community variation in plant biomass allocation: A balance between organ biomass and morphology above vs below ground?Journal of Vegetation Science, 26(3), 431-440. https://doi.org/10.1111/ jvs.12259

Freschet, G. T., Swart, E. M., \& Cornelissen, J. H. C. (2015). Integrated plant phenotypic responses to contrasting above- and below-ground resources: Key roles of specific leaf area and root mass fraction. New Phytologist, 206(4), 1247-1260. https://doi.org/10.1111/nph.13352

Freschet, G. T., Violle, C., Bourget, M. Y., Scherer-Lorenzen, M., \& Fort, F. (2018). Allocation, morphology, physiology, architecture: The multiple facets of plant above- and below-ground responses to resource stress. New Phytologist, 219(4), 1338-1352. https://doi.org/10.1111/nph.15225

Fry, E. L., Power, S. A., \& Manning, P. (2013). Trait-based classification and manipulation of plant functional groups for biodiversity-ecosystem function experiments. Journal of Vegetation Science, 25(1), 248-261. https://doi.org/10.1111/jvs.12068

Fyllas, N. M., Christopoulou, A., Galanidis, A., Michelaki, C. Z., Giannakopoulos, C., Dimitrakopoulos, P. G., ... Gloor, M. (2017). Predicting species dominance shifts across elevation gradients in mountain forests in Greece under a warmer and drier climate. Regional Environmental Change, 17(4), 1165-1177. https://doi.org/10.1007/ s10113-016-1093-1

Fyllas, N. M., Patiño, S., Baker, T. R., Bielefeld Nardoto, G., Martinelli, L. A., Quesada, C. A., ... Lloyd, J. (2009). Basin-wide variations in foliar properties of Amazonian forest: Phylogeny, soils and climate. Biogeosciences, 6(11), 2677-2708. https://doi.org/10.5194/ bg-6-2677-2009

Gachet, S., Véla, E., \& Tatoni, T. (2005). BASECO: A floristic and ecological database of Mediterranean French flora. Biodiversity and Conservation, 14(4), 1023-1034. https://doi.org/10.1007/s10531-004-8411-5

Gallagher, R. V., \& Leishman, M. R. (2012). A global analysis of trait variation and evolution in climbing plants. Journal of Biogeography, 39(10), 1757-1771. https://doi.org/10.1111/j.1365-2699.2012.02773.x

Gallagher, R. V., Leishman, M. R., \& Moles, A. T. (2011). Traits and ecological strategies of Australian tropical and temperate climbing plants. Journal of Biogeography, 38(5), 828-839. https://doi. org/10.1111/j.1365-2699.2010.02455.x
García-Palacios, P., Maestre, F. T., Kattge, J., \& Wall, D. H. (2013). Climate and litter quality differently modulate the effects of soil fauna on litter decomposition across biomes. Ecology Letters, 16(8), 1045-1053. https://doi.org/10.1111/ele.12137

Garnier, E., Lavorel, S., Ansquer, P., Castro, H., Cruz, P., Dolezal, J., ... Zarovali, M. P. (2007). Assessing the effects of land-use change on plant traits, communities and ecosystem functioning in grasslands: A standardized methodology and lessons from an application to 11 European sites. Annals of Botany, 99(5), 967-985. https://doi. org/10.1093/aob/mcl215

Giarrizzo, E., Burrascano, S., Chiti, T., de Bello, F., Lepš, J., Zavattero, L., \& Blasi, C. (2016). Re-visiting historical semi-natural grasslands in the Apennines to assess patterns of changes in species composition and functional traits. Applied Vegetation Science, 20(2), 247-258. https://doi.org/10.1111/avsc.12288

Giroldo, A. (2016). Pequenas plantas, grandes estrategias: adaptacoes e sobrevivencia no Cerrado. PhD thesis, University of Brasilia, Brasil.

Givnish, T. J., Montgomery, R. A., \& Goldstein, G. (2004). Adaptive radiation of photosynthetic physiology in the Hawaiian lobeliads: Light regimes, static light responses, and whole-plant compensation points. American Journal of Botany, 91(2), 228-246. https://doi.org/10.3732/ ajb.91.2.228

Golovko, T., Dymova, O., Yatsko, Y., \& Tabalenkova, G. (2011). Photosynthetic pigments apparatus in the northern plants. In $\mathrm{M}$. Pessarakli (Ed.), Handbook of plant and crop stress (3rd ed., pp. 391405). New York, NY: Marcel Dekker.

Gonzalez-Akre, E., McShea, W., Bourg, N., \& Anderson-Teixeira, K. (2015). Leaf traits data (SLA) for 56 woody species at the Smithsonian Conservation Biology Institute-ForestGEO Forest Dynamic Plot. Front Royal, Virginia. USA. [Data set]. Version 1.0. Retrieved from http:// www.try-db.org

Granda, E., Baumgarten, F., Gessler, A., Gil-Pelegrin, E., Peguero-Pina, J. J., Sancho-Knapik, D. E., ... Resco de Dios, V. (2020). Day-length regulates seasonal patterns of stomatal conductance in Quercus species. Plant, Cell \& Environment. https://doi.org/10.1111/pce.13665

Gubsch, M., Buchmann, N., Schmid, B., Schulze, E.-D., Lipowsky, A., \& Roscher, C. (2011). Differential effects of plant diversity on functional trait variation of grass species. Annals of Botany, 107(1), 157169. https://doi.org/10.1093/aob/mcq220

Guerin, G. R., Wen, H., \& Lowe, A. J. (2012). Leaf morphology shift linked to climate change. Biology Letters, 8(5), 882-886. https://doi. org/10.1098/rsbl.2012.0458

Gutiérrez, A. G., \& Huth, A. (2012). Successional stages of primary temperate rainforests of Chiloé Island, Chile. Perspectives in Plant Ecology, Evolution and Systematics, 14(4), 243-256. https://doi.org/10.1016/j. ppees.2012.01.004

Guy, A. L., Mischkolz, J. M., \& Lamb, E. G. (2013). Limited effects of simulated acidic deposition on seedling survivorship and root morphology of endemic plant taxa of the Athabasca Sand Dunes in well-watered greenhouse trials. Botany-Botanique, 91(3), 176-181. https://doi. org/10.1139/cjb-2012-0162

Han, W., Fang, J., Guo, D., \& Zhang, Y. (2005). Leaf nitrogen and phosphorus stoichiometry across 753 terrestrial plant species in China. New Phytologist, 168(2), 377-385. https://doi.org/10.1111/j.14698137.2005.01530.x

Han, W. X., Chen, Y. H., Zhao, F. J., Tang, L. Y., Jiang, R. F., \& Zhang, F. S. (2012). Floral, climatic and soil pH controls on leaf ash content in China's terrestrial plants. Global Ecology and Biogeography, 21(3), 376382. https://doi.org/10.1111/j.1466-8238.2011.00677.x

Hayes, F. J., Buchanan, S. W., Coleman, B., Gordon, A. M., Reich, P. B., Thevathasan, N. V., ... Martin, A. R. (2018). Intraspecific variation in soy across the leaf economics spectrum. Annals of Botany, 123(1), 107-120. https://doi.org/10.1093/aob/mcy147

He, P., Wright, I. J., Zhu, S., Onoda, Y., Liu, H., Li, R., ... Ye, Q. (2019). Leaf mechanical strength and photosynthetic capacity vary independently 
across 57 subtropical forest species with contrasting light requirements. New Phytologist, 223(2), 607-618. https://doi.org/10.1111/ nph.15803

He, T., Fowler, W. M., \& Causley, C. L. (2015). High nutrient-use efficiency during early seedling growth in diverse Grevillea species (Proteaceae). Scientific Reports, 5. https://doi.org/10.1038/srep17132

He, T., Lamont, B. B., \& Downs, K. S. (2011). Banksias born to burn. New Phytologist, 191, 184-196. https://doi.org/10.1111/j.1469-8137.2011. 03663.x

He,T.,Pausas, J.P., Belcher, C.M.,Schwilk,D.W.,\&Lamont, B. B. (2012). Fireadapted traits of Pinus arose in the fiery Cretaceous. New Phytologist, 194, 751-759. https://doi.org/10.1111/j.1469-8137.2012.04079.x

Heberling, J. M., Cassidy, S. T., Fridley, J. D., \& Kalisz, S. (2019). Carbon gain phenologies of spring-flowering perennials in a deciduous forest indicate a novel niche for a widespread invader. New Phytologist, 221(2), 778-788. https://doi.org/10.1111/nph.15404

Heberling, J. M., \& Mason, N. W. H. (2018). Are endemics functionally distinct? Leaf traits of native and exotic woody species in a New Zealand forest. PLoS ONE, 13(5), e0196746. https://doi.org/10.1371/ journal.pone.0196746

Herz, K., Dietz, S., Haider, S., Jandt, U., Scheel, D., \& Bruelheide, H. (2017). Drivers of intraspecific trait variation of grass and forb species in German meadows and pastures. Journal of Vegetation Science, 28(4), 705-716. https://doi.org/10.1111/jvs.12534

Hickler, T. (1999). Plant functional types and community characteristics along environmental gradients on Öland's Great Alvar (Sweden). Master's thesis, University of Lund, Sweden.

Hietz, P., Rosner, S., Hietz-Seifert, U., \& Wright, S. J. (2016). Wood traits related to size and life history of trees in a Panamanian rainforest. New Phytologist, 213(1), 170-180. https://doi.org/10.1111/ nph.14123

Hill, M. O., Preston, C. D., \& Roy, D. B. (2004). PLANTATT - Attributes of British and Irish plants: Status, size, life history, geography and habitats. Huntingdon, UK: Centre for Ecology and Hydrology.

Hipp, A. L., Glasenhardt, M.-C., Bowles, M. L., Garner, M., Scharenbroch, B. C., Williams, E. W., ... Larkin, D. J. (2018). Effects of phylogenetic diversity and phylogenetic identity in a restoration ecology experiment. In R. Scherson \& D. Faith (Eds.), Phylogenetic diversity (pp. 189210). Cham: Springer.

Hogan, J. A., Valverde-Barrantes, O. J., Ding, Q., Xu, H., \& Baraloto, C. (2019). Intraspecific root and leaf trait variation with tropical forest successional status: Consequences for community-weighted patterns. Retrieved from http://dx.doi.org/10.1101/611640

Hou, E., Chen, C., McGroddy, M. E., \& Wen, D. (2012). Nutrient limitation on ecosystem productivity and processes of mature and old-growth subtropical forests in China. PLoS ONE, 7(12), e52071. https://doi. org/10.1371/journal.pone.0052071

Hough-Snee, N., Nackley, L. L., Kim, S.-H., \& Ewing, K. (2015). Does plant performance under stress explain divergent life history strategies? The effects of flooding and nutrient stress on two wetland sedges. Aquatic Botany, 120, 151-159. https://doi.org/10.1016/j.aquabot. 2014.03.001

Isaac, M. E., Martin, A. R., de Melo Virginio Filho, E., Rapidel, B., Roupsard, O., \& Van den Meersche, K. (2017). Intraspecific trait variation and coordination: Root and leaf economics spectra in coffee across environmental gradients. Frontiers in Plant Science, 8(1196), https://doi. org/10.3389/fpls.2017.01196

Jager, M. M., Richardson, S. J., Bellingham, P. J., Clearwater, M. J., \& Laughlin, D. C. (2015). Soil fertility induces coordinated responses of multiple independent functional traits. Journal of Ecology, 103(2), 374-385. https://doi.org/10.1111/1365-2745.12366

Joseph, G. S., Seymour, C. L., Cumming, G. S., Cumming, D. H. M., \& Mahlangu, Z. (2014). Termite mounds increase functional diversity of woody plants in African savannas. Ecosystems, 17(5), 808-819. https://doi.org/10.1007/s10021-014-9761-9
Kaplan, Z., J. Danihelka, J. Chrtek , J. Kirschner, K. Kubát, M. Štech, \& J. Štěpánek (Eds.) (2019). Klíč ke květeně České republiky [Key to the flora of the Czech Republic] (2nd ed.). Academia, Praha: Czech Republic.

Kapralov, M. V., Smith, J. A. C., \& Filatov, D. A. (2012). Rubisco evolution in C4 eudicots: An analysis of Amaranthaceae Sensu Lato. PLoS ONE, 7(12), e52974. https://doi.org/10.1371/journal.pone.0052974

Kattenborn, T., Fassnacht, F. E., \& Schmidtlein, S. (2018). Differentiating plant functional types using reflectance: Which traits make the difference?Remote Sensing in Ecology and Conservation, 5(1), 5-19. https://doi.org/10.1002/rse2.86

Kattenborn, T., \& Schmidtlein, S. (2019). Radiative transfer modelling reveals why canopy reflectance follows function. Scientific Reports, 9(1). https://doi.org/10.1038/s41598-019-43011-1

Kattge, J., Knorr, W., Raddatz, T., \& Wirth, C. (2009). Quantifying photosynthetic capacity and its relationship to leaf nitrogen content for global-scale terrestrial biosphere models. Global Change Biology, 15(4), 976-991. https://doi.org/10.1111/j.1365-2486.2008.01744.x

Kavelenova, L. M., Rozno, S. A., Kireyeva, Y. V., \& Smirnov, Y. V. (2007). $\mathrm{K}$ strukturno-funktsional'nym osobennostyam list'yev drevesnykh rasteniy $v$ nasazhdeniyakh lesostepi. Byulleten' Samarskaya Luka, 16: 3(21), 568-574.

Kearsley, E., Verbeeck, H., Hufkens, K., Van de Perre, F., Doetterl, S., Baert, G., ... Huygens, D. (2016). Functional community structure of African monodominant Gilbertiodendron dewevrei forest influenced by local environmental filtering. Ecology and Evolution, 7(1), 295-304. https://doi.org/10.1002/ece3.2589

Kempel, A., Chrobock, T., Fischer, M., Rohr, R. P., \& van Kleunen, M. (2013). Determinants of plant establishment success in a multispecies introduction experiment with native and alien species. Proceedings of the National Academy of Sciences of the United States of America, 110(31), 12727-12732. https://doi.org/10.1073/pnas.1300481110

Kerkhoff, A. J., Fagan, W. F., Elser, J. J., \& Enquist, B. J. (2006). Phylogenetic and growth form variation in the scaling of nitrogen and phosphorus in the seed plants. The American Naturalist, 168(4), E103-E122. https://doi.org/10.1086/507879

Khalil, M. I., Gibson, D. J., Baer, S. G., \& Willand, J. E. (2018). Functional diversity is more sensitive to biotic filters than phylogenetic diversity during community assembly. Ecosphere, 9(3), e02164.https://doi. org/10.1002/ecs2.2164

Kichenin, E., Wardle, D. A., Peltzer, D. A., Morse, C. W., \& Freschet, G. T. (2013). Contrasting effects of plant inter- and intraspecific variation on community-level trait measures along an environmental gradient. Functional Ecology, 27(5), 1254-1261. https://doi. org/10.1111/1365-2435.12116

Kirkup, D., Malcolm, P., Christian, G., \& Paton, A. (2005). Towards a digital African flora. Taxon, 54(2), 457-466. https://doi.org/10.2307/ 25065373

Kisel, Y., Moreno-Letelier, A. C., Bogarín, D., Powell, M. P., Chase, M. W., \& Barraclough, T. G. (2012). Testing the link between population genetic differentiation and clade diversification in Costa Rican orchids. Evolution, 66(10), 3035-3052. https://doi.org/10.1111/ j.1558-5646.2012.01663.x

Kissling, W. D., Balslev, H., Baker, W. J., Dransfield, J., Göldel, B., Lim, J. Y., ... Svenning, J.-C. (2019). PalmTraits 1.0, a species-level functional trait database of palms worldwide. Scientific Data, 6(1), 178. https://doi.org/10.1038/s41597-019-0189-0

Klein, T., Di Matteo, G., Rotenberg, E., Cohen, S., \& Yakir, D. (2012). Differential ecophysiological response of a major Mediterranean pine species across a climatic gradient. Tree Physiology, 33(1), 26-36. https://doi.org/10.1093/treephys/tps116

Klimešová, J., \& de Bello, F. (2009). CLO-PLA: The database of clonal and bud bank traits of Central European flora. Journal of Vegetation Science, 20(3), 511-516. https://doi.org/10.1111/j.1654-1103.2009.01050.x

Knauer, J., Zaehle, S., Medlyn, B. E., Reichstein, M., Williams, C. A., Migliavacca, M., ... Linderson, M. L. (2017). Towards physiologically meaningful 
water-use efficiency estimates from eddy covariance data. Global Change Biology, 24(2), 694-710. https://doi.org/10.1111/gcb.13893

Koele, N., Dickie, I. A., Oleksyn, J., Richardson, S. J., \& Reich, P. B. (2012). No globally consistent effect of ectomycorrhizal status on foliar traits. New Phytologist, 196(3), 845-852. https://doi. org/10.1111/j.1469-8137.2012.04297.x

Koike, F. (2001). Plant traits as predictors of woody species dominance in climax forest communities. Journal of Vegetation Science, 12(3), 327336. https://doi.org/10.2307/3236846

Komac, B., Pladevall, C., Domènech, M., \& Fanlo, R. (2014). Functional diversity and grazing intensity in sub-alpine and alpine grasslands in Andorra. Applied Vegetation Science, 18(1), 75-85. https://doi. org/10.1111/avsc.12119

Kraft, N. J. B., Valencia, R., \& Ackerly, D. D. (2008). Functional traits and niche-based tree community assembly in an Amazonian forest. Science, 322(5901), 580-582. https://doi.org/10.1126/scien ce. 1160662

Kumarathunge, D. P., Medlyn, B. E., Drake, J. E., Tjoelker, M. G., Aspinwall, M. J., Battaglia, M., ... Way, D. A. (2019). Acclimation and adaptation components of the temperature dependence of plant photosynthesis at the global scale. New Phytologist, 222(2), 768-784. https://doi. org/10.1111/nph.15668

Kuppler, J., Höfers, M. K., Trutschnig, W., Bathke, A. C., Eiben, J. A., Daehler, C. C., \& Junker, R. R. (2017). Exotic flower visitors exploit large floral trait spaces resulting in asymmetric resource partitioning with native visitors. Functional Ecology, 31(12), 2244-2254. https://doi.org/10.1111/1365-2435.12932

Kurokawa, H., \& Nakashizuka, T. (2008). Leaf herbivory and decomposability in a Malaysian tropical rain forest. Ecology, 89(9), 2645-2656. https://doi.org/10.1890/07-1352.1

La Pierre, K. J., \& Smith, M. D. (2014). Functional trait expression of grassland species shift with short- and long-term nutrient additions. Plant Ecology, 216(2), 307-318. https://doi.org/10.1007/ s11258-014-0438-4

Laughlin, D. C., Fulé, P. Z., Huffman, D. W., Crouse, J., \& Laliberté, E. (2011). Climatic constraints on trait-based forest assembly. Journal of Ecology, 99(6), 1489-1499. https://doi.org/10.1111/j.1365-2745. 2011.01885.x

Lavergne, S., \& Molofsky, J. (2007). Increased genetic variation and evolutionary potential drive the success of an invasive grass. Proceedings of the National Academy of Sciences of the United States of America, 104(10), 3883-3888. https://doi.org/10.1073/pnas.0607324104

Lavorel, S., Grigulis, K., Lamarque, P., Colace, M.-P., Garden, D., Girel, J., ... Douzet, R. (2010). Using plant functional traits to understand the landscape distribution of multiple ecosystem services. Journal of Ecology, 99(1), 135-147. https://doi.org/10.1111/j.1365-2745.2010. 01753.x

Lens, F., Endress, M. E., Baas, P., Jansen, S., \& Smets, E. (2008). Wood anatomy of Rauvolfioideae (Apocynaceae): A search for meaningful non-DNA characters at the tribal level. American Journal of Botany, 95, 1199-1215. https://doi.org/10.3732/ajb.0800159

Lens, F., Gasson, P., Smets, E., \& Jansen, S. (2003). Comparative wood anatomy of epacrids (Styphelioideae, Ericaceae s.I.). Annals of Botany, 91, 835-857. https://doi.org/10.1093/aob/mcg089

Lens, F., Sperry, J. S., Christman, M. A., Choat, B., Rabaey, D., \& Jansen, S. (2011). Testing hypotheses that link wood anatomy to cavitation resistance and hydraulic conductivity in the genus Acer. New Phytologist, 190, 709-723. https://doi.org/10.1111/j.1469-8137.2010.03518.x

Letts, B., Lamb, E. G., Mischkolz, J. M., \& Romo, J. T. (2015). Litter accumulation drives grassland plant community composition and functional diversity via leaf traits. Plant Ecology, 216, 357-370. https://doi. org/10.1007/s11258-014-0436-6

Lhotsky, B., Csecserits, A., Kovács, B., \& Botta-Dukát, Z. (2016). New plant trait records of the Hungarian flora. Acta Botanica Hungarica, 58(3-4), 397-400. https://doi.org/10.1556/abot.58.2016.3-4.8
Li, R., Zhu, S., Chen, H. Y. H., John, R., Zhou, G., Zhang, D., ... Ye, Q. (2015). Are functional traits a good predictor of global change impacts on tree species abundance dynamics in a subtropical forest?Ecology Letters, 18(11), 1181-1189. https://doi.org/10.1111/ele.12497

Li, X., Nie, Y., Song, X., Zhang, R., \& Wang, G. (2011). Patterns of species diversity and functional diversity along a south-to north-facing slope in a sub-alpine meadow. Community Ecology, 12(2), 179-187.

Li, Y., \& Shipley, B. (2018). Community divergence and convergence along experimental gradients of stress and disturbance. Ecology, 99(4), 775781. https://doi.org/10.1002/ecy.2162

Liebergesell, M., Reu, B., Stahl, U., Freiberg, M., Welk, E., Kattge, J., ... Wirth, C. (2016). Functional resilience against climate-driven extinctions - Comparing the functional diversity of European and North American tree floras. PLoS ONE, 11(2), e0148607. https://doi. org/10.1371/journal.pone.0148607

Lin, Y.-S., Medlyn, B. E., Duursma, R. A., Prentice, I. C., Wang, H., Baig, S., ... Wingate, L. (2015). Optimal stomatal behaviour around the world. Nature Climate Change, 5(5), 459-464. https://doi.org/10.1038/nclim ate 2550

Lipowsky, A., Roscher, C., Schumacher, J., Michalski, S., Gubsch, M., Buchmann, N., ... Schmid, B. (2015). Plasticity of functional traits of forb species in response to biodiversity. Perspectives in Plant Ecology, Evolution and Systematics, 17, 66-77. https://doi.org/10.1016/j.ppees. 2014.11.003

Lohbeck, M., Poorter, L., Paz, H., Pla, L., van Breugel, M., MartínezRamos, M., \& Bongers, F. (2012). Functional diversity changes during tropical forest succession. Perspectives in Plant Ecology, Evolution and Systematics, 14(2), 89-96. https://doi.org/10.1016/ j.ppees.2011.10.002

Louault, F., Pillar, V. D., Aufrère, J., Garnier, E., \& Soussana, J. F. (2005). Plant traits and functional types in response to reduced disturbance in a semi-natural grassland. Journal of Vegetation Science, 16(2), 151. https://doi.org/10.1658/1100-9233(2005)016[0151:ptafti]2.0.co;2

Lukeš, P., Stenberg, P., Rautiainen, M., Mõttus, M., \& Vanhatalo, K. M. (2013). Optical properties of leaves and needles for boreal tree species in Europe. Remote Sensing Letters, 4(7), 667-676. https://doi. org/10.1080/2150704x.2013.782112

Lusk, C. H. (2019). Leaf functional trait variation in a humid temperate forest, and relationships with juvenile tree light requirements. PeerJ, 7, e6855. https://doi.org/10.7717/peerj.6855

Lusk, C. H., Kaneko, T., Grierson, E., \& Clearwater, M. (2013). Correlates of tree species sorting along a temperature gradient in New Zealand rain forests: Seedling functional traits, growth and shade tolerance. Journal of Ecology, 101(6), 1531-1541. https://doi. org/10.1111/1365-2745.12152

Maire, V., Wright, I. J., Prentice, I. C., Batjes, N. H., Bhaskar, R., van Bodegom, P. M., ... Santiago, L. S. (2015). Global effects of soil and climate on leaf photosynthetic traits and rates. Global Ecology and Biogeography, 24(6), 706-717. https://doi.org/10.1111/geb.12296

Malhado, A. C. M., Malhi, Y., Whittaker, R. J., Ladle, R. J., terSteege, H., Fabré, N. N., ... Malhado, C. H. M. (2012). Drip-tips are associated with intensity of precipitation in the Amazon rain forest. Biotropica, 44(6), 728-737. https://doi.org/10.1111/j.1744-7429.2012.00868.x

Malhado, A. C. M., Malhi, Y., Whittaker, R. J., Ladle, R. J., terSteege, H., Phillips, O. L., ... Laurance, W. F. (2009). Spatial trends in leaf size of Amazonian rainforest trees. Biogeosciences, 6(8), 1563-1576. https://doi.org/10.5194/bg-6-1563-2009

Malhado, A. C. M., Whittaker, R. J., Malhi, Y., Ladle, R. J., terSteege, H., Butt, N., ... Ramírez, A. H., (2009). Spatial distribution and functional significance of leaf lamina shape in Amazonian forest trees. Biogeosciences, 6(8), 1577-1590. https://doi.org/10.5194/ bg-6-1577-2009

Malhado, A. C. M., Whittaker, R. J., Malhi, Y., Ladle, R. J., Ter Steege, H., Phillips, O., ... Ramírez-Angulo, H. (2010). Are compound leaves an adaptation to seasonal drought or to rapid growth? Evidence from the 
Amazon rain forest. Global Ecology and Biogeography, 19(6), 852-862. https://doi.org/10.1111/j.1466-8238.2010.00567.x

Manning, P., Newington, J. E., Robson, H. R., Saunders, M., Eggers, T., Bradford, M. A., ... Rees, M. (2006). Decoupling the direct and indirect effects of nitrogen deposition on ecosystem function. Ecology Letters, 9(9), 1015-1024. https://doi.org/10.1111/j.1461-0248.2006. 00959.x

Manzoni, S., Vico, G., Porporato, A., \& Katul, G. (2013). Biological constraints on water transport in the soil-plant-atmosphere system. Advances in Water Resources, 51, 292-304. https://doi.org/10.1016/ j.advwatres.2012.03.016

Martin, A. R., Doraisami, M., \& Thomas, S. C. (2018). Global patterns in wood carbon concentration across the world's trees and forests. Nature Geoscience, 11(12), 915-920. https://doi.org/10.1038/ s41561-018-0246-x

Martin, A. R., Hayes, F. J., Borden, K. A., Buchanan, S. W., Gordon, A. M., Isaac, M. E., \& Thevathasan, N. V. (2019). Integrating nitrogen fixing structures into above- and belowground functional trait spectra in soy (Glycine max). Plant and Soil, 440(1-2), 53-69. https://doi. org/10.1007/s11104-019-04058-1

Martin, A. R., Rapidel, B., Roupsard, O., Van den Meersche, K., de Melo Virginio Filho, E., Barrios, M., \& Isaac, M. E. (2017). Intraspecific trait variation across multiple scales: The leaf economics spectrum in coffee. Functional Ecology, 31(3), 604-612. https://doi. org/10.1111/1365-2435.12790

Martínez-Garza, C., Bongers, F., \& Poorter, L. (2013). Are functional traits good predictors of species performance in restoration plantings in tropical abandoned pastures?Forest Ecology and Management, 303, 35-45. https://doi.org/10.1016/j.foreco.2013.03.046

Messier, J., Violle, C., Enquist, B. J., Lechowicz, M. J., \& B, McGill, J. (2018). Similarities and differences in intrapopulation trait correlations of co-occurring tree species: Consistent water use relationships amidst widely different correlation patterns. American Journal of Botany, 105(9), 1-14. https://doi.org/10.1002/ajb2.1146

McCarthy, J. K., Dwyer, J. M., \& Mokany, K. (2019). A regional-scale assessment of using metabolic scaling theory to predict ecosystem properties. Proceedings of the Royal Society B: Biological Sciences, 286. https://doi.org/10.1098/rspb.2019.2221

McFadden, I. R., Bartlett, M. K., Wiegand, T., Turner, B. L., Sack, L., Valencia, R., \& Kraft, N. J. B. (2019). Disentangling the functional trait correlates of spatial aggregation in tropical forest trees. Ecology, 100(3), e02591. https://doi.org/10.1002/ecy.2591

Medeiros, J. S., Burns, J. H., Nicholson, J., Rogers, L., \& Valverde-Barrantes, O. (2017). Decoupled leaf and root carbon economics is a key component in the ecological diversity and evolutionary divergence of deciduous and evergreen lineages of genus Rhododendron. American Journal of Botany, 104(6), 803-816. https://doi.org/10.3732/ajb.1700051

Medlyn, B. E., Badeck, F. W., De Pury, D. G. G., Barton, C. V. M., Broadmeadow, M., Ceulemans, R., ... Jstbid, P. G. (1999). Effects of elevated [CO2] on photosynthesis in European forest species: A meta-analysis of model parameters. Plant, Cell \& Environment, 22(12), 1475-1495. https://doi.org/10.1046/j.1365-3040.1999.00523.x

Meir, P., Kruijt, B., Broadmeadow, M., Barbosa, E., Kull, O., Carswell, F., ... Jarvis, P. G. (2002). Acclimation of photosynthetic capacity to irradiance in tree canopies in relation to leaf nitrogen concentration and leaf mass per unit area. Plant, Cell and Environment, 25(3), 343-357. https://doi.org/10.1046/j.0016-8025.2001.00811.x

Mencuccini, M. (2003). The ecological significance of long-distance water transport:Short-term regulation, long-term acclimation and the hydraulic costs of stature across plant life forms. Plant, Cell and Environment, 26(1), 163-182. https://doi.org/10.1046/j.1365-3040.2003.00991.x

Messier, J., McGill, B. J., \& Lechowicz, M. J. (2010). How do traits vary across ecological scales? A case for trait-based ecology. Ecology Letters, 13(7), 838-848. https://doi.org/10.1111/j.1461-0248.2010.01476.x
Messier, J., Violle, C., Enquist, B. J., Lechowicz, M. J., \& McGill, B. J. (2018). Similarities and differences in intrapopulation trait correlations of co-occurring tree species: Consistent water use relationships amidst widely different correlation patterns. American Journal of Botany, 105(9), 1-14. https://doi.org/10.1002/ajb2.1146

Michaletz, S. T., \& Johnson, E. A. (2006). A heat transfer model of crown scorch in forest fires. Canadian Journal of Forest Research, 36(11), 2839-2851. https://doi.org/10.1139/x06-158

Michelaki, C., Fyllas, N. M., Galanidis, A., Aloupi, M., Evangelou, E., Arianoutsou, M., \& Dimitrakopoulos, P. G. (2019). An integrated phenotypic trait-network in thermo-Mediterranean vegetation describing alternative, coexisting resource-use strategies. Science of the Total Environment, 672, 583-592. https://doi.org/10.1016/j.scitotenv.2019.04.030

Milla, R., \& Reich, P. B. (2011). Multi-trait interactions, not phylogeny, fine-tune leaf size reduction with increasing altitude. Annals of Botany, 107(3), 455-465. https://doi.org/10.1093/aob/mcq261

Miller, J. E. D., Ives, A. R., Harrison, S. P., \& Damschen, E. I. (2017). Early- and late-flowering guilds respond differently to landscape spatial structure. Journal of Ecology, 106(3), 1033-1045. https://doi. org/10.1111/1365-2745.12849

Minden, V., Deloy, A., Volkert, A. M., Leonhardt, S. D., \& Pufal, G. (2017). Antibiotics impact plant traits, even at small concentrations. AoB PLANTS, 9(2). https://doi.org/10.1093/aobpla/plx010

Minden, V., \& Gorschlüter, J. (2016). Comparison of native and non-native Impatiens species across experimental light and nutrient gradients. Plant Ecology and Evolution, 149(1), 59-72. https://doi.org/10.5091/ plecevo.2016.1118

Minden, V., \& Kleyer, M. (2011). Testing the effect-response framework: Key response and effect traits determining above-ground biomass of salt marshes. Journal of Vegetation Science, 22(3), 387-401. https://doi.org/10.1111/j.1654-1103.2011.01272.x

Minden, V., \& Kleyer, M. (2014). Internal and external regulation of plant organ stoichiometry. Plant Biology, 16(5), 897-907. https://doi.org/ 10.1111/plb.12155

Minden, V., \& Kleyer, M. (2015). Ecosystem multifunctionality of coastal marshes is determined by key plant traits. Journal of Vegetation Science, 26(4), 651-662. https://doi.org/10.1111/jvs.12276

Minden, V., \& Olde Venterink, H. (2019). Plant traits and species interactions along gradients of $\mathrm{N}, \mathrm{P}$ and $\mathrm{K}$ availabilities. Functional Ecology, 33(9), 1611-1626. https://doi.org/10.1111/1365-2435.13387

Minden, V., Schnetger, B., Pufal, G., \& Leonhardt, S. D. (2018). Antibioticinduced effects on scaling relationships and on plant element contents in herbs and grasses. Ecology and Evolution, 8(13), 6699-6713. https://doi.org/10.1002/ece3.4168

Moles, A. T., Ackerly, D. D., Webb, C. O., Tweddle, J. C., Dickie, J. B., Pitman, A. J., \& Westoby, M. (2005). Factors that shape seed mass evolution. Proceedings of the National Academy of Sciences of the United States of America, 102(30), 10540-10544. https://doi.org/10.1073/ pnas.0501473102

Moles, A. T., Warton, D. I., Warman, L., Swenson, N. G., Laffan, S. W., Zanne, A. E., ... Leishman, M. R. (2009). Global patterns in plant height. Journal of Ecology, 97, 923-932. https://doi.org/10.1111/ j.1365-2745.2009.01526.x

Moravcová, L., Pyšek, P., Jarošík, V., Havlíčková, V., \& Zákravský, P. (2010). Reproductive characteristics of neophytes in the Czech Republic: Traits of invasive and non-invasive species. Preslia, 82, 365-390.

Moretti, M., \& Legg, C. (2009). Combining plant and animal traits to assess community functional responses to disturbance. Ecography, 32(2), 299-309. https://doi.org/10.1111/j.1600-0587.2008.05524.x

Mori, A. S., Shiono, T., Haraguchi, T. F., Ota, A. T., Koide, D., Ohgue, T., ... Gustafsson, L. (2015). Functional redundancy of multiple forest taxa along an elevational gradient: Predicting the consequences of non-random species loss. Journal of Biogeography, 42(8), 1383-1396. https://doi.org/10.1111/jbi.12514 
Muir, C. D., Hangarter, R. P., Moyle, L. C., \& Davis, P. A. (2013). Morphological and anatomical determinants of mesophyll conductance in wild relatives of tomato (Solanum sect. Lycopersicon, sect. Lycopersicoides; Solanaceae). Plant, Cell \& Environment, 37(6), 14151426. https://doi.org/10.1111/pce.12245

Müller, S. C., Overbeck, G. E., Pfadenhauer, J., \& Pillar, V. D. (2006). Plant functional types of woody species related to fire disturbance in forest-grassland ecotones. Plant Ecology, 189(1), 1-14. https://doi. org/10.1007/s11258-006-9162-z

Nakahashi, C. D., Frole, K., \& Sack, L. (2005). Bacterial leaf nodule symbiosis in Ardisia (Myrsinaceae): Does it contribute to seedling growth capacity? Plant Biology, 7(5), 495-500. https://doi.org/10.1055/s-2005-865853

Neuschulz, E. L., Mueller, T., Schleuning, M., \& Böhning-Gaese, K. (2016). Pollination and seed dispersal are the most threatened processes of plant regeneration. Scientific Reports, 6. https://doi.org/10.1038/ srep29839

Niinemets, Ü. (2001). Global-scale climatic controls of leaf dry mass per area, density, and thickness in trees and shrubs. Ecology, 82(2), 453. https://doi.org/10.2307/2679872

Niinemets, Ü., \& Valladares, F. (2006). Tolerance to shade, drought, and waterlogging of temperate northern hemisphere trees and shrubs. Ecological Monographs, 76(4), 521-547. https://doi.org/10.1890/001 2-9615(2006)076[0521:ttsdaw]2.0.co;2

Nolan, R. H., Fairweather, K. A., Tarin, T., Santini, N. S., Cleverly, J., Faux, R., \& Eamus, D. (2017). Divergence in plant water-use strategies in semiarid woody species. Functional Plant Biology, 44(11), 1134. https://doi.org/10.1071/fp17079

Nolan, R. H., Hedo, J., Arteaga, C., Sugai, T., \& Resco de Dios, V. (2018). Physiological drought responses improve predictions of live fuel moisture dynamics in a Mediterranean forest. Agricultural and Forest Meteorology, 263, 417-427. https://doi.org/10.1016/j.agrformet.2018.09.011

Núñez-Florez, R., Pérez-Gómez, U., \& Fernández-Méndez, F. (2019). Functional diversity criteria for selecting urban trees. Urban Forestry \& Urban Greening, 38, 251-266. https://doi.org/10.1016/ j.ufug.2019.01.005

Ogaya, R., \& Peñuelas, J. (2003). Comparative field study of Quercus ilex and Phillyrea latifolia: Photosynthetic response to experimental drought conditions. Environmental and Experimental Botany, 50(2), 137-148. https://doi.org/10.1016/s0098-8472(03)00019-4

Oliveira, R. A. C., Marques, R., \& Marques, M. C. M. (2019). Plant diversity and local environmental conditions indirectly affect litter decomposition in a tropical forest. Applied Soil Ecology, 134, 45-53. https://doi. org/10.1016/j.apsoil.2018.09.016

Olson, M. E., Anfodillo, T., Rosell, J. A., Petit, G., Crivellaro, A., Isnard, S., ... Castorena, M. (2014). Universal hydraulics of the flowering plants: Vessel diameter scales with stem length across angiosperm lineages, habits and climates. Ecology Letters, 17(8), 988-997. https://doi. org/10.1111/ele.12302

Olson, M. E., Rosell, J. A., Zamora Muñoz, S., \& Castorena, M. (2018). Carbon limitation, stem growth rate and the biomechanical cause of Corner's rules. Annals of Botany, 122(4), 583-592. https://doi. org/10.1093/aob/mcy089

Olson, M. E., Soriano, D., Rosell, J. A., Anfodillo, T., Donoghue, M. J., Edwards, E. J., ... Méndez-Alonzo, R. (2018). Plant height and hydraulic vulnerability to drought and cold. Proceedings of the National Academy of Sciences of the United States of America, 115(29), 75517556. https://doi.org/10.1073/pnas.1721728115

Onoda, Y., Westoby, M., Adler, P. B., Choong, A. M. F., Clissold, F. J., Cornelissen, J. H. C., ... Yamashita, N. (2011). Global patterns of leaf mechanical properties. Ecology Letters, 14(3), 301-312. https://doi. org/10.1111/j.1461-0248.2010.01582.x

Onoda, Y., Wright, I. J., Evans, J. R., Hikosaka, K., Kitajima, K., Niinemets, Ü., ... Westoby, M. (2017). Physiological and structural tradeoffs underlying the leaf economics spectrum. New Phytologist, 214(4), 14471463. https://doi.org/10.1111/nph.14496
Onstein, R. E., Carter, R. J., Xing, Y., \& Linder, H. P. (2014). Diversification rate shifts in the Cape Floristic Region: The right traits in the right place at the right time. Perspectives in Plant Ecology, Evolution and Systematics, 16(6), 331-340. https://doi.org/10.1016/j.ppees.2014.08.002

Ordoñez, J. C., vanBodegom, P. M., Witte, J.-P. M., Bartholomeus, R. P, van Hal, J. R., \& Aerts, R. (2010). Plant strategies in relation to resource supply in mesic to wet environments: Does theory mirror nature? The American Naturalist, 175(2), 225-239. https://doi. org/10.1086/649582

OReilly-Nugent, A., Wandrag, E., Catford, J., Gruber, B., Driscoll, D., \& Duncan, R. (2019). Measuring competitive impact: Joint-species modelling of invaded plant communities. Journal of Ecology. https://doi. org/10.1111/1365-2745.13280

Ostonen, I., Rosenvald, K., Helmisaari, H.-S., Godbold, D., Parts, K., Uri, V., \& Lõhmus, K. (2013). Morphological plasticity of ectomycorrhizal short roots in Betula sp and Picea abies forests across climate and forest succession gradients: Its role in changing environments. Frontiers in Plant Science, 4. https://doi.org/10.3389/fpls.2013.00335

Ostonen, I., Tedersoo, L., Suvi, T., \& Lõhmus, K. (2009). Does a fungal species drive ectomycorrhizal root traits in Alnus spp.? Canadian Journal of Forest Research, 39(10), 1787-1796. https://doi.org/10.1139/x09-093

Ottaviani, G., Marcantonio, M., \& Mucina, L. (2016). Soil depth shapes plant functional diversity in granite outcrops vegetation of Southwestern Australia. Plant Ecology \& Diversity, 9(3), 263-276. https://doi.org/10.1080/17550874.2016.1211192

Pahl, A. T., Kollmann, J., Mayer, A., \& Haider, S. (2013). No evidence for local adaptation in an invasive alien plant: Field and greenhouse experiments tracing a colonization sequence. Annals of Botany, 112(9), 1921-1930. https://doi.org/10.1093/aob/mct246

Paine, C. E. T., Amissah, L., Auge, H., Baraloto, C., Baruffol, M., Bourland, N., ... Hector, A. (2015). Globally, functional traits are weak predictors of juvenile tree growth, and we do not know why. Journal of Ecology, 103(4), 978-989. https://doi.org/10.1111/1365-2745.12401

Paule, J., Gregor, T., Schmidt, M., Gerstner, E.-M., Dersch, G., Dressler, S., ... Zizka, G. (2017). Chromosome numbers of the flora of Germany-A new online database of georeferenced chromosome counts and flow cytometric ploidy estimates. Plant Systematics and Evolution, 303(8), 1123-1129. https://doi.org/10.1007/s00606-016-1362-y

Pausas, J. G., Lamont, B. B., Paula, S., Appezzato-da-Glória, B., \& Fidelis, A. (2018). Unearthing belowground bud banks in fire-prone ecosystems. New Phytologist, 217(4), 1435-1448. https://doi.org/10.1111/ nph.14982

Pausas, J. G., Pratt, R. B., Keeley, J. E., Jacobsen, A. L., Ramirez, A. R., Vilagrosa, A., ... Davis, S. D. (2015). Towards understanding resprouting at the global scale. New Phytologist, 209(3), 945-954. https://doi. org/10.1111/nph.13644

Peco, B., dePablos, I., Traba, J., \& Levassor, C. (2005). The effect of grazing abandonment on species composition and functional traits: The case of dehesa grasslands. Basic and Applied Ecology, 6(2), 175-183. https://doi.org/10.1016/j.baae.2005.01.002

Peñuelas, J., Sardans, J., Filella, I., Estiarte, M., Llusià, J., Ogaya, R., ... Terradas, J. (2017). Impacts of global change on Mediterranean forests and their services. Forests, 8(12), 463. https://doi.org/10.3390/ f8120463

Peñuelas, J., Sardans, J., Filella, I., Estiarte, M., Llusià, J., Ogaya, R., ... Terradas, J. (2018). Assessment of the impacts of climate change on Mediterranean terrestrial ecosystems based on data from field experiments and long-term monitored field gradients in Catalonia. Environmental and Experimental Botany, 152, 49-59. https://doi. org/10.1016/j.envexpbot.2017.05.012

Peñuelas, J., Sardans, J., Llusia, J., Owen, S. M., Carnicer, J., Giambelluca, T. W., ... Niinemets, Ü. (2009). Faster returns on 'leaf economics' and different biogeochemical niche in invasive compared with native plant species. Global Change Biology, 16(8), 2171-2185. https://doi. org/10.1111/j.1365-2486.2009.02054.x 
Petter, G., Wagner, K., Wanek, W., Sánchez Delgado, E. J., Zotz, G., Cabral, J. S., \& Kreft, H. (2016). Functional leaf traits of vascular epiphytes: Vertical trends within the forest, intra- and interspecific trait variability, and taxonomic signals. Functional Ecology, 30(2), 188-198. https://doi.org/10.1111/1365-2435.12490

Pierce, S., Brusa, G., Sartori, M., \& Cerabolini, B. E. L. (2012). Combined use of leaf size and economics traits allows direct comparison of hydrophyte and terrestrial herbaceous adaptive strategies. Annals of Botany, 109(5), 1047-1053. https://doi.org/10.1093/aob/mcs021

Pierce, S., Ceriani, R. M., De Andreis, R., Luzzaro, A., \& Cerabolini, B. (2007). The leaf economics spectrum of Poaceae reflects variation in survival strategies. Plant Biosystems, 141(3), 337-343. https://doi. org/10.1080/11263500701627695

Pierce, S., Vagge, I., Brusa, G., \& Cerabolini, B. E. L. (2014). The intimacy between sexual traits and Grime's CSR strategies for orchids coexisting in semi-natural calcareous grassland at the Olive Lawn. Plant Ecology, 215(5), 495-505. https://doi.org/10.1007/s11258-014-0318-y

Pinho, B. X., deMelo, F. P. L., Arroyo-Rodríguez, V., Pierce, S., Lohbeck, M., \& Tabarelli, M. (2017). Soil-mediated filtering organizes tree assemblages in regenerating tropical forests. Journal of Ecology, 106(1), 137-147. https://doi.org/10.1111/1365-2745.12843

Pisek, J., Sonnentag, O., Richardson, A. D., \& Mõttus, M. (2013). Is the spherical leaf inclination angle distribution a valid assumption for temperate and boreal broadleaf tree species?Agricultural and Forest Meteorology, 169, 186-194. https://doi.org/10.1016/j.agrformet.2012.10.011

Pomogaybin, A. V., \& Pomogaybin, Y. A. Kizucheniyu bioekologicheskikh osobennostey predstaviteley roda Juglans L. pri introduktsii $v$ lesostepi Srednego Povolzhya Sovremennaya botanika v Rossii. Trudy XIII syezda Russkogo botanicheskogo obshchestva (pp. 156-158).

Poorter, H., Jagodzinski, A. M., Ruiz-Peinado, R., Kuyah, S., Luo, Y., Oleksyn, J., ... Sack, L. (2015). How does biomass distribution change with size and differ among species? An analysis for 1200 plant species from five continents. New Phytologist, 208, 736-749. https://doi. org/10.1111/nph.13571

Poorter, H., Niinemets, Ü., Poorter, L., Wright, I. J., \& Villar, R. (2009). Causes and consequences of variation in leaf mass per area (LMA): A meta-analysis. New Phytologist, 182(3), 565-588. https://doi. org/10.1111/j.1469-8137.2009.02830.x

Poorter, L., \& Bongers, F. (2006). Leaf traits are good predictors of plant performance across 53 rain forest species. Ecology, 87(7), 17331743. https://doi.org/10.1890/0012-9658(2006)87[1733:Itagpo]2. $0 . c 0 ; 2$

Powers, J. S., \& Tiffin, P. (2010). Plant functional type classifications in tropical dry forests in Costa Rica: Leaf habit versus taxonomic approaches. Functional Ecology, 24(4), 927-936. https://doi. org/10.1111/j.1365-2435.2010.01701.x

Prentice, I. C., Meng, T., Wang, H., Harrison, S. P., Ni, J., \& Wang, G. (2010). Evidence of a universal scaling relationship for leaf $\mathrm{CO}_{2}$ drawdown along an aridity gradient. New Phytologist, 190(1), 169-180. https://doi.org/10.1111/j.1469-8137.2010.03579.x

Preston, K. A., Cornwell, W. K., \& DeNoyer, J. L. (2006). Wood density and vessel traits as distinct correlates of ecological strategy in 51 California coast range angiosperms. New Phytologist, 170(4), 807818. https://doi.org/10.1111/j.1469-8137.2006.01712.x

Price, C. A., \& Enquist, B. J. (2007). Scaling mass and morphology in leaves: An extension of the WBE model. Ecology, 88(5), 1132-1141. https://doi.org/10.1890/06-1158

Puglielli, G., \& Varone, L. (2018). Inherent variation of functional traits in winter and summer leaves of Mediterranean seasonal dimorphic species: Evidence of a 'within leaf cohort' spectrum. AoB PLANTS, 10(3). https://doi.org/10.1093/aobpla/ply027

Purcell, A. S. T., Lee, W. G., Tanentzap, A. J., \& Laughlin, D. C. (2018). Fine root traits are correlated with flooding duration while aboveground traits are related to grazing in an ephemeral wetland. Wetlands, 39(2), 291-302. https://doi.org/10.1007/s13157-018-1084-8
Quitián, M., Santillán, V., Espinosa, C. I., Homeier, J., Böhning-Gaese, K., Schleuning, M., \& Neuschulz, E. L. (2018). Direct and indirect effects of plant and frugivore diversity on structural and functional components of fruit removal by birds. Oecologia, 189(2), 435-445. https://doi.org/10.1007/s00442-018-4324-y

Raabe, K., Pisek, J., Sonnentag, O., \& Annuk, K. (2015). Variations of leaf inclination angle distribution with height over the growing season and light exposure for eight broadleaf tree species. Agricultural and Forest Meteorology, 214-215, 2-11. https://doi.org/10.1016/j.agrfo rmet.2015.07.008

Raevel, V., Anthelme, F., Meneses, R. I., \& Munoz, F. (2018). Cushion-plant protection determines guild-dependent plant strategies in high-elevation peatlands of the Cordillera Real, Bolivian Andes. Perspectives in Plant Ecology, Evolution and Systematics, 30, 103-114. https://doi. org/10.1016/j.ppees.2017.09.006

Raevel, V., Violle, C., \& Munoz, F. (2012). Mechanisms of ecological succession: Insights from plant functional strategies. Oikos, 121(11), 1761-1770. https://doi.org/10.1111/j.1600-0706.2012.20261.x

Reich, P. B., Oleksyn, J., \& Wright, I. J. (2009). Leaf phosphorus influences the photosynthesis-nitrogen relation: A cross-biome analysis of 314 species. Oecologia, 160(2), 207-212. https://doi.org/10.1007/ s00442-009-1291-3

Reich, P. B., Tjoelker, M. G., Pregitzer, K. S., Wright, I. J., Oleksyn, J., \& Machado, J.-L. (2008). Scaling of respiration to nitrogen in leaves, stems and roots of higher land plants. Ecology Letters, 11(8), 793-801. https://doi.org/10.1111/j.1461-0248.2008.01185.x

Richardson, S. J., Allen, R. B., Buxton, R. P., Easdale, T. A., Hurst, J. M., Morse, C. W., ... Peltzer, D. A. (2013). Intraspecific relationships among wood density, leaf structural traits and environment in four co-occurring species of Nothofagus in New Zealand. PLoS ONE, 8(3), e58878. https://doi.org/10.1371/journal.pone.0058878

Richardson, S. J., Laughlin, D. C., Lawes, M. J., Holdaway, R. J., Wilmshurst, J. M., Wright, M., ... McGlone, M. S. (2015). Functional and environmental determinants of bark thickness in fire-free temperate rain forest communities. American Journal of Botany, 102(10), 1590-1598. https://doi.org/10.3732/ajb.1500157

Richardson, S. J., Williams, P. A., Mason, N. W. H., Buxton, R. P., Courtney, S. P., Rance, B. D., ... Wiser, S. K. (2012). Rare species drive local trait diversity in two geographically disjunct examples of a naturally rare alpine ecosystem in New Zealand. Journal of Vegetation Science, 23(4), 626-639. https://doi.org/10.1111/j.1654-1103.2012.01396.x

Roddy, A. B., Jiang, G., Cao, K., Simonin, K. A., \& Brodersen, C. R. (2019). Hydraulic traits are more diverse in flowers than in leaves. New Phytologist, 223, 193-203. https://doi.org/10.1111/nph.15749

Rodrigues, A., Bones, F., Schneiders, A., Oliveira, L., Vibrans, A., \& Gasper, A. (2018). Plant trait dataset for tree-like growth forms species of the subtropical Atlantic rain forest in Brazil. Data, 3(2), 16. https://doi. org $/ 10.3390 /$ data3020016

Rogers, A., Serbin, S. P., Ely, K. S., Sloan, V. L., \& Wullschleger, S. D. (2017). Terrestrial biosphere models underestimate photosynthetic capacity and $\mathrm{CO}_{2}$ assimilation in the Arctic. New Phytologist, 216(4), 1090-1103. https://doi.org/10.1111/nph.14740

Rolo, V., López-Díaz, M. L., \& Moreno, G. (2012). Shrubs affect soil nutrients availability with contrasting consequences for pasture understory and tree overstory production and nutrient status in Mediterranean grazed open woodlands. Nutrient Cycling in Agroecosystems, 93(1), 89-102. https://doi.org/10.1007/s10705-012-9502-4

Rolo, V., Olivier, P., \& van Aarde, R. (2016). Seeded pioneer die-offs reduce the functional trait space of new-growth coastal dune forests. Forest Ecology and Management, 377, 26-35. https://doi.org/10.1016/j. foreco.2016.06.039

Ronzhina, D. A., \& P'Yankov, V. I. (2001). Structure of the photosynthetic apparatus in leaves of freshwaterhydrophytes: 2. Quantitative characterization of leaf mesophyll and the functional activity of leaves with different degrees of submersion. Russian Journal of 
Plant Physiology, 48(6), 723-732. https://doi.org/10.1023/a:10125 44105453

Roscher, C., Schmid, B., Buchmann, N., Weigelt, A., \& Schulze, E.D. (2011). Legume species differ in the responses of their functional traits to plant diversity. Oecologia, 165, 437-452. https://doi. org/10.1007/s00442-010-1735-9

Rosell, J. A. (2016). Bark thickness across the angiosperms: More than just fire. New Phytologist, 211(1), 90-102. https://doi.org/10.1111/ nph.13889

Rosell, J. A., Gleason, S., Méndez-Alonzo, R., Chang, Y., \& Westoby, M. (2013). Bark functional ecology: Evidence for tradeoffs, functional coordination, and environment producing bark diversity. New Phytologist, 201(2), 486-497. https://doi.org/10.1111/nph.12541

Rosell, J. A., \& Olson, M. E. (2014). Do lianas really have wide vessels? Vessel diameter-stem length scaling in non-self-supporting plants. Perspectives in Plant Ecology, Evolution and Systematics, 16(6), 288295. https://doi.org/10.1016/j.ppees.2014.08.001

Rosell, J. A., Olson, M. E., Anfodillo, T., \& Martínez-Méndez, N. (2017). Exploring the bark thickness-stem diameter relationship: Clues from lianas, successive cambia, monocots and gymnosperms. New Phytologist, 215(2), 569-581. https://doi.org/10.1111/nph.14628

Rossi, C. (2017). Three morphological plant traits in the Swiss National Park and surroundings. Retrieved from http://www.parcs.ch/snp/mmd_ fullentry.php?docu_xml:id=37905

Royal Botanic Gardens Kew. (2019). Seed Information Database (SID). Version 7.1. Retrieved from http://data.kew.org/sid/

Rüger, N., Berger, U., Hubbell, S. P., Vieilledent, G., \& Condit, R. (2011). Growth strategies of tropical tree species: Disentangling light and size effects. PLoS ONE, 6(9), e25330. https://doi.org/10.1371/journ al.pone. 0025330

Rüger, N., Huth, A., Hubbell, S. P., \& Condit, R. (2009). Response of recruitment to light availability across a tropical lowland rain forest community. Journal of Ecology, 97(6), 1360-1368. https://doi. org/10.1111/j.1365-2745.2009.01552.x

Rüger, N., Huth, A., Hubbell, S. P., \& Condit, R. (2011). Determinants of mortality across a tropical lowland rainforest community. Oikos, 120(7), 1047-1056. https://doi.org/10.1111/j.1600-0706.2010.19021.x

Rumpf, S. B., Hülber, K., Klonner, G., Moser, D., Schütz, M., Wessely, J., ... Dullinger, S. (2018). Range dynamics of mountain plants decrease with elevation. Proceedings of the National Academy of Sciences of the United States of America, 115(8), 1848-1853. https://doi.org/10.1073/ pnas.1713936115

Sanda, V., Bita-Nicolae, C. D., \& Barabas, N. (2003). The flora of spontane and cultivated cormophytes from Romania (in Romanian) (p. 316). Bacău: Editura Ion Borcea.

Sandel, B., Corbin, J. D., \& Krupa, M. (2011). Using plant functional traits to guide restoration: A case study in California coastal grassland. Ecosphere, 2(2), art23. https://doi.org/10.1890/es10-00175.1

Scalon, M. C., Haridasan, M., \& Franco, A. C. (2017). Influence of longterm nutrient manipulation on specific leaf area and leaf nutrient concentrations in savanna woody species of contrasting leaf phenologies. Plant and Soil, 421(1-2), 233-244. https://doi.org/10.1007/ s11104-017-3437-0

Schall, P., Lödige, C., Beck, M., \& Ammer, C. (2012). Biomass allocation to roots and shoots is more sensitive to shade and drought in European beech than in Norway spruce seedlings. Forest Ecology and Management, 266, 246-253. https://doi.org/10.1016/j.foreco. 2011.11.017

Scherer-Lorenzen, M., Schulze, E., Don, A., Schumacher, J., \& Weller, E. (2007). Exploring the functional significance of forest diversity: A new long-term experiment with temperate tree species (BIOTREE). Perspectives in Plant Ecology, Evolution and Systematics, 9(2), 53-70. https://doi.org/10.1016/j.ppees.2007.08.002

Schmitt, M., Mehltreter, K., Sundue, M., Testo, W., Watanabe, T., \& Jansen, S. (2017). The evolution of aluminum accumulation in ferns and lycophytes. American Journal of Botany, 104(4), 573-583. https://doi.org/10.3732/ajb.1600381

Schroeder-Georgi, T., Wirth, C., Nadrowski, K., Meyer, S. T., Mommer, L., $\&$ Weigelt, A. (2015). From pots to plots: Hierarchical trait-based prediction of plant performance in a mesic grassland. Journal of Ecology, 104(1), 206-218. https://doi.org/10.1111/1365-2745.12489

Schuldt, B., Leuschner, C., Brock, N., \& Horna, V. (2013). Changes in wood density, wood anatomy and hydraulic properties of the xylem along the root-to-shoot flow path in tropical rainforest trees. Tree Physiology, 33(2), 161-174. https://doi.org/10.1093/treephys/tps122

Schurr, F. M., Midgley, G. F., Rebelo, A. G., Reeves, G., Poschlod, P., \& Higgins, S. I. (2007). Colonization and persistence ability explain the extent to which plant species fill their potential range. Global Ecology and Biogeography, 16(4), 449-459. https://doi. org/10.1111/j.1466-8238.2006.00293.x

Schweingruber, F. H., \& Landolt, W. (2005). The xylem database (updated). Birmensdorf, Switzerland: Swiss Federal Research Institute, WSL, Birmensdorf, Switzerland.

Seymour, C. L., Milewski, A. V., Mills, A. J., Joseph, G. S., Cumming, G. S., Cumming, D. H. M., \& Mahlangu, Z. (2014). Do the large termite mounds of Macrotermes concentrate micronutrients in addition to macronutrients in nutrient-poor African savannas? Soil Biology and Biochemistry, 68, 95-105. https://doi.org/10.1016/j.soilbio.2013.09.022

Sfair, J. C., deBello, F., deFrança, T. Q., Baldauf, C., \& Tabarelli, M. (2018). Chronic human disturbance affects plant trait distribution in a seasonally dry tropical forest. Environmental Research Letters, 13(2), 025005. https://doi.org/10.1088/1748-9326/aa9f5e

Sheremetiev, S. N., \& Chebotareva, K. E. (2018). Modern and cretaceouscenozoic diversification of angiosperms. Biology Bulletin Reviews, 8(5), 351-374. https://doi.org/10.1134/s2079086418050079

Shiodera, S., Rahajoe, J. S., \& Kohyama, T. (2008). Variation in longevity and traits of leaves among co-occurring understorey plants in a tropical montane forest. Journal of Tropical Ecology, 24(2), 121-133. https://doi.org/10.1017/s0266467407004725

Shipley, B. (2002). Trade-offs between net assimilation rate and specific leaf area in determining relative growth rate: Relationship with daily irradiance. Functional Ecology, 16(5), 682-689. https://doi. org/10.1046/j.1365-2435.2002.00672.x

Shovon, T., Rozendaal, D., Gagnon, D., Gendron, F., Vetter, M., \& Vanderwel, M. (2019). Plant communities on nitrogen-rich soil are less sensitive to soil moisture than plant communities on nitrogen-poor soil. Journal of Ecology. https://doi.org/10.1111/1365-2745.13251

Siebenkäs, A., Schumacher, J., \& Roscher, C. (2015). Phenotypic plasticity to light and nutrient availability alters functional trait ranking across eight perennial grassland species. AoB PLANTS, 7, 1-15. https://doi. org/10.1093/aobpla/plv029

Siefert, A. (2011). Spatial patterns of functional divergence in oldfield plant communities. Oikos, 121(6), 907-914. https://doi. org/10.1111/j.1600-0706.2011.19706.x

Siefert, A., Fridley, J. D., \& Ritchie, M. E. (2014). Community functional responses to soil and climate at multiple spatial scales: When does intraspecific variation matter?PLoS ONE, 9(10), e111189. https://doi. org/10.1371/journal.pone.0111189

Silva, M. C., Teodoro, G. S., Bragion, E. F. A., \& van denBerg, E. (2019). The role of intraspecific trait variation in the occupation of sharp forest-savanna ecotones. Flora, 253, 35-42. https://doi.org/10.1016/j. flora.2019.03.003

Silva, V., Catry, F. X., Fernandes, P. M., Rego, F. C., Paes, P., Nunes, L., ... Bugalho, M. N. (2019). Effects of grazing on plant composition, conservation status and ecosystem services of Natura 2000 shrub-grassland habitat types. Biodiversity and Conservation, 28(5), 1205-1224. https://doi.org/10.1007/s10531-019-01718-7

Sitzia, T., Dainese, M., Krüsi, B. O., \& McCollin, D. (2017). Landscape metrics as functional traits in plants: Perspectives from a glacier foreland. PeerJ, 5, e3552. https://doi.org/10.7717/peerj.3552 
Sitzia, T., Michielon, B., lacopino, S., \& Kotze, D. J. (2016). Population dynamics of the endangered shrub Myricaria germanica in a regulated Alpine river is influenced by active channel width and distance to check dams. Ecological Engineering, 95, 828-838. https://doi. org/10.1016/j.ecoleng.2016.06.066

Sjöman, H., Hirons, A. D., \& Bassuk, N. L. (2015). Urban forest resilience through tree selection-Variation in drought tolerance in Acer. Urban Forestry \& Urban Greening, 14(4), 858-865. https://doi.org/10.1016/j. ufug.2015.08.004

Slot, M., Rey-Sánchez, C., Winter, K., \& Kitajima, K. (2014). Trait-based scaling of temperature-dependent foliar respiration in a species-rich tropical forest canopy. Functional Ecology, 28(5), 1074-1086. https://doi.org/10.1111/1365-2435.12263

Slot, M., \& Winter, K. (2017). In situ temperature response of photosynthesis of 42 tree and liana species in the canopy of two Panamanian lowland tropical forests with contrasting rainfall regime. New Phytologist, 214(3), 1103-1117. https://doi.org/10.1111/nph.14469

Smith, N. G., \& Dukes, J. S. (2017). LCE: Leaf carbon exchange data set for tropical, temperate, and boreal species of North and Central America. Ecology, 98(11), 2978-2978. https://doi.org/10.1002/ecy.1992

Smith, N. G., Pold, G., Goranson, C., \& Dukes, J. S. (2016). Characterizing the drivers of seedling leaf gas exchange responses to warming and altered precipitation: Indirect and direct effects. AoB PLANTS, 8, plw066. https://doi.org/10.1093/aobpla/plw066

Smith, S. W., Woodin, S. J., Pakeman, R. J., Johnson, D., \& van derWal, R. (2014). Root traits predict decomposition across a landscape-scale grazing experiment. New Phytologist, 203(3), 851-862. https://doi. org/10.1111/nph.12845

Soboleski, V. F., Higuchi, P., Silva, A. C. D., Loebens, R., Souza, K., Buzzi Junior, F., ... Dallabrida, J. P. (2017). Variação de atributos funcionais do componente arbóreo em função de gradientes edáficos em uma floresta nebular no sul do Brasil. Rodriguésia, 68(2), 291-300. https://doi.org/10.1590/2175-7860201768201

Sodhi, D. S., Livingstone, S. W., Carboni, M., \& Cadotte, M. W. (2019). Plant invasion alters trait composition and diversity across habitats. Ecology and Evolution, 9, 6199-6210. https://doi.org/10.1002/ece3.5130

Soler Martin, M., Bonet, J. A., Martínez De Aragón, J., Voltas, J., Coll, L., \& Resco De Dios, V. (2017). Crown bulk density and fuel moisture dynamics in Pinus pinaster stands are neither modified by thinning nor captured by the Forest Fire Weather Index. Annals of Forest Science, 74. https://doi.org/10.1007/s13595-017-0650-1

Soudzilovskaia, N. A., Elumeeva, T. G., Onipchenko, V. G., Shidakov, I. I., Salpagarova, F. S., Khubiev, A. B., ... Cornelissen, J. H. C. (2013). Functional traits predict relationship between plant abundance dynamic and long-term climate warming. Proceedings of the National Academy of Sciences of the United States of America, 110(45), 1818018184. https://doi.org/10.1073/pnas.1310700110

Souza, K., Higuchi, P., Silva, A. C. D., Schimalski, M. B., Loebens, R., Buzzi Júnior, F., ... Rosa, A. D. (2017). Partição de nicho por grupos funcionais de espécies arbóreas em uma floresta subtropical. Rodriguésia, 68(4), 1165-1175. https://doi.org/10.1590/2175-7860201768401

Spasojevic, M. J., \& Suding, K. N. (2012). Inferring community assembly mechanisms from functional diversity patterns: The importance of multiple assembly processes. Journal of Ecology, 100(3), 652-661. https://doi.org/10.1111/j.1365-2745.2011.01945.x

Spasojevic, M. J., Turner, B. L., \& Myers, J. A. (2016). When does intraspecific trait variation contribute to functional beta-diversity? Journal of Ecology, 104(2), 487-496. https://doi.org/10.1111/13652745.12518

Staples, T. L., Dwyer, J. M., England, J. R., \& Mayfield, M. M. (2019). Productivity does not correlate with species and functional diversity in Australian reforestation plantings across a wide climate gradient. Global Ecology and Biogeography, 28(10), 1417-1429. https://doi. org/10.1111/geb.12962
Steyn, C., Greve, M., Robertson, M. P., Kalwij, J. M., \& leRoux, P. C. (2016). Alien plant species that invade high elevations are generalists: Support for the directional ecological filtering hypothesis. Journal of Vegetation Science, 28(2), 337-346. https://doi.org/10.1111/jvs.12477

Swaine, E. K. (2007). Ecological and evolutionary drivers of plant community assembly in a Bornean rain forest. PhD thesis, University of Aberdeen, Aberdeen.

Swenson, N. G., Anglada-Cordero, P., \& Barone, J. A. (2010). Deterministic tropical tree community turnover: Evidence from patterns of functional beta diversity along an elevational gradient. Proceedings of the Royal Society B: Biological Sciences, 278(1707), 877-884. https://doi. org/10.1098/rspb.2010.1369

Takkis, K. (2014). Changes in plant species richness and population performance in response to habitat loss and fragmentation. PhD, University Tartu. Retrieved from http://hdl.handle.net/10062/ 39546 (Dissertationes Biologicae Universitatis Tartuensis 255, 2014-04-07.).

Tavşanoğlu, Ç., \& Pausas, J. G. (2018). A functional trait database for Mediterranean Basin plants. Scientific Data, 5. https://doi. org/10.1038/sdata.2018.135

Tedersoo, L., Laanisto, L., Rahimlou, S., Toussaint, A., Hallikma, T., \& Pärtel, M. (2018). Global database of plants with root-symbiotic nitrogen fixation: NodDB. Journal of Vegetation Science, 29(3), 560-568. https://doi.org/10.1111/jvs.12627

The Tree of Sex Consortium, Ashman, T.-L., Bachtrog, D., Blackmon, H., Goldberg, E., Hahn, M., ... Vamosi, J. (2014). Tree of sex: A database of sexual systems. Scientific Data, 1, 140015. https://doi.org/10.1038/ sdata. 2014.15

Thomas, E., Alcazar, C., Moscoso, H. L. G., Osorio, L. F., Salgado, B., Gonzalez, M., ... Ramirez, W. (2017). The importance of species selection and seed sourcing in forest restoration for enhancing adaptive potential to climate change: Colombian tropical dry forest as a model. CBD Technical Series, 89, 122-134.

Thomas, S. C., \& Martin, A. R. (2012). Wood carbon content database. Retrieved from http://dx.doi.org/10.5061/dryad.69sg2

Tng, D. Y. P., Jordan, G. J., \& Bowman, D. M. J. S. (2013). Plant traits demonstrate that temperate and tropical giant eucalypt forests are ecologically convergent with rainforest not savanna. PLoS ONE, 8(12), e84378. https://doi.org/10.1371/journal.pone.0084378

Torca, M., Campos, J. A., \& Herrera, M. (2019). Species composition and plant traits of south Atlantic European coastal dunes and other comparative data. Data in Brief, 22, 207-213. https://doi.org/10.1016/j. dib.2018.12.005

Torres-Ruiz, J. M., Cochard, H., Fonseca, E., Badel, E., Gazarini, L., \& Vaz, M. (2017). Differences in functional and xylem anatomical features allow Cistus species to co-occur and cope differently with drought in the Mediterranean region. Tree Physiology, 37(6), 755-766. https://doi. org/10.1093/treephys/tpx013

Tribouillois, H., Fort, F., Cruz, P., Charles, R., Flores, O., Garnier, E., \& Justes, E. (2015). A functional characterisation of a wide range of cover crop species: Growth and nitrogen acquisition rates, leaf traits and ecological strategies. PLoS ONE, 10(3), e0122156. https://doi. org/10.1371/journal.pone.0122156

Usoltsev, V. (2010). Фитомасса и первичная продукция лесов Евразии = Eurasian forest biomass and primary production data / B. A. Усольцев ; [отв. ред. С. Г. Шиятов]; Рос. акад. наук, Урал. отд-ние, Ботан. сад УрО РАН, Урал. гос. лесотехн. ун-т. - Екатеринбург: УрО РАН, 570 с. - Парал. тит. англ. - Библиогр.: с. 520.

van Bodegom, P. M., Sorrell, B. K., Oosthoek, A., Bakke, C., \& Aerts, R. (2008). Separating the effects of partial submergence and soil oxygen demand on plant physiology. Ecology, 89(1), 193-204. https://doi. org/10.1890/07-0390.1

Van Cleemput, E., Roberts, D. A., Honnay, O., \& Somers, B. (2019). A novel procedure for measuring functional traits of herbaceous 
species through field spectroscopy. Methods in Ecology and Evolution, 10(8), 1332-1338. https://doi.org/10.1111/2041-210x.13237

van deWeg, M. J., Meir, P., Grace, J., \& Atkin, O. K. (2009). Altitudinal variation in leaf mass per unit area, leaf tissue density and foliar nitrogen and phosphorus content along an Amazon-Andes gradient in Peru. Plant Ecology \& Diversity, 2(3), 243-254. https://doi. org/10.1080/17550870903518045

van deWeg, M. J., Meir, P., Grace, J., \& Ramos, G. D. (2011). Photosynthetic parameters, dark respiration and leaf traits in the canopy of a Peruvian tropical montane cloud forest. Oecologia, 168(1), 23-34. https://doi. org/10.1007/s00442-011-2068-z

Van der Plas, F., Howison, R., Reinders, J., Fokkema, W., \& Olff, H. (2013). Functional traits of trees on and off termite mounds: Understanding the origin of biotically-driven heterogeneity in savannas. Journal of Vegetation Science, 24(2), 227-238. https://doi. org/10.1111/j.1654-1103.2012.01459.x

van derSande, M. T., Arets, E. J. M. M., Peña-Claros, M., Hoosbeek, M. R., Cáceres-Siani, Y., van derHout, P., \& Poorter, L. (2017). Soil fertility and species traits, but not diversity, drive productivity and biomass stocks in a Guyanese tropical rainforest. Functional Ecology, 32(2), 461-474. https://doi.org/10.1111/1365-2435.12968

Vanselow, K. A., Samimi, C., \& Breckle, S.-W. (2016). Preserving a comprehensive vegetation knowledge base - An evaluation of four historical soviet vegetation maps of the Western Pamirs (Tajikistan). PLoS ONE, 11(2), e0148930. https://doi.org/10.1371/journal.pone.0148930

Vásquez-Valderrama, M. (2016). Efecto de especies con potencial invasor en procesos de regulación hídrica del suelo en un ecosistema seco tropical. Maestría en Manejo, Uso y Conservación del Bosque, Universidad Distrital Francisco José de Caldas, Bogotá, Colombia.

Vassilev, K., Pedashenko, H., Nikolov, S. C., Apostolova, I., \& Dengler, J. (2011). Effect of land abandonment on the vegetation of upland semi-natural grasslands in the Western Balkan Mts., Bulgaria. Plant Biosystems, 145(3), 654-665. https://doi.org/10.1080/11263 504.2011.601337

Verdier, B., Jouanneau, I., Simonnet, B., Rabin, C., Van Dooren, T. J. M., Delpierre, N., ... Le Galliard, J.-F. (2014). Climate and atmosphere simulator for experiments on ecological systems in changing environments. Environmental Science \& Technology, 48(15), 8744-8753. https://doi.org/10.1021/es405467s

Vergutz, L., Manzoni, S., Porporato, A., Novais, R. F., \& Jackson, R. B. (2012). Global resorption efficiencies and concentrations of carbon and nutrients in leaves of terrestrial plants. Ecological Monographs, 82(2), 205-220. https://doi.org/10.1890/11-0416.1

Von Holle, B., \& Simberloff, D. (2004). Testing Fox's assembly rule: Does plant invasion depend on recipient community structure?Oikos, 105(3), 551-563. https://doi.org/10.1111/j.0030-1299.2004.12597.x

Wagenführ, R. (2007). Holzatlas (Vol. 6, neu bearbeitete und erweiterte Auflage). Leipzig, Germany: Fachbuchverlag Leipzig.

Walker, A. P., Beckerman, A. P., Gu, L., Kattge, J., Cernusak, L. A., Domingues, T. F., ... Woodward, F. I. (2014). The relationship of leaf photosynthetic traits $-\mathrm{V}_{\mathrm{cmax}}$ and $\mathrm{J}_{\max }$ - To leaf nitrogen, leaf phosphorus, and specific leaf area: A meta-analysis and modeling study. Ecology and Evolution, 4(16), 3218-3235. https://doi.org/10.1002/ece3.1173

Wang, H., Harrison, S. P., Prentice, I. C., Yang, Y., Bai, F., Togashi, H. F., ... $\mathrm{Ni}$, J. (2018). The China plant trait database: Toward a comprehensive regional compilation of functional traits for land plants. Ecology, 99(2), 500-500. https://doi.org/10.1002/ecy.2091

Watanabe, T., Broadley, M. R., Jansen, S., White, P. J., Takada, J., Satake, K., ... Osaki, M. (2007). Evolutionary control of leaf element composition in plants. New Phytologist, 174(3), 516-523. https://doi. org/10.1111/j.1469-8137.2007.02078.x

Weedon, J. T., Cornwell, W. K., Cornelissen, J. H. C., Zanne, A. E., Wirth, C., \& Coomes, D. A. (2009). Global meta-analysis of wood decomposition rates: A role for trait variation among tree species?Ecology Letters, 12(1), 45-56. https://doi.org/10.1111/j.1461-0248.2008.01259.x
Wellstein, C., Chelli, S., Campetella, G., Bartha, S., Galiè, M., Spada, F., \& Canullo, R. (2013). Intraspecific phenotypic variability of plant functional traits in contrasting mountain grasslands habitats. Biodiversity and Conservation, 22(10), 2353-2374. https://doi.org/ 10.1007/s10531-013-0484-6

Werner, G. D. A., Cornelissen, J. H. C., Cornwell, W. K., Soudzilovskaia, N. A., Kattge, J., West, S. A., \& Kiers, E. T. (2018). Symbiont switching and alternative resource acquisition strategies drive mutualism breakdown. Retrieved from http://dx.doi.org/10.1101/242834

Werner, G. D. A., Cornwell, W. K., Sprent, J. I., Kattge, J., \& Kiers, E. T. (2014). A single evolutionary innovation drives the deep evolution of symbiotic $\mathrm{N}_{2}$-fixation in angiosperms. Nature Communications, 5(1), 4087. https://doi.org/10.1038/ncomms5087

White, M. A., Thornton, P. E., Running, S. W., \& Nemani, R. R. (2000). Parameterization and sensitivity analysis of the BIOME-BGC terrestrial ecosystem model: Net primary production controls. Earth Interactions, 4(3), 1-85. https://doi.org/10.1175/1087-3562(2000)004<0003:pasao $\mathrm{t}>2.0 . \mathrm{co} ; 2$

White, P. J., Broadley, M. R., Thompson, J. A., McNicol, J. W., Crawley, M. J., Poulton, P. R., \& Johnston, A. E. (2012). Testing the distinctness of shoot ionomes of angiosperm families using the Rothamsted Park Grass Continuous Hay Experiment. New Phytologist, 196(1), 101-109. https://doi.org/10.1111/j.1469-8137.2012.04228.x

Williams, G. M., \& Nelson, A. S. (2018). Spatial variation in specific leaf area and horizontal distribution of leaf area in juvenile western larch (Larix occidentalis Nutt.). Trees, 32(6), 1621-1631. https://doi. org/10.1007/s00468-018-1738-4

Williams, M., Shimabokuro, Y. E., \& Rastetter, E. B. (2012). LBAECO CD-09 Soil and Vegetation Characteristics, Tapajos National Forest, Brazil. Retrieved from http://dx.doi.org/10.3334/ORNLD AAC $/ 1104$

Willis, C. G., Halina, M., Lehman, C., Reich, P. B., Keen, A., McCarthy, S., \& Cavender-Bares, J. (2010). Phylogenetic community structure in Minnesota oak savanna is influenced by spatial extent and environmental variation. Ecography, 33, 565-577. https://doi. org/10.1111/j.1600-0587.2009.05975.x

Wilson, K. B., Baldocchi, D. D., \& Hanson, P. J. (2000). Spatial and seasonal variability of photosynthetic parameters and their relationship to leaf nitrogen in a deciduous forest. Tree Physiology, 20(9), 565-578. https://doi.org/10.1093/treephys/20.9.565

Winkler, D. E., Amagai, Y., Huxman, T. E., Kaneko, M., \& Kudo, G. (2016). Seasonal dry-down rates and high stress tolerance promote bamboo invasion above and below treeline. Plant Ecology, 217(10), 1219-1234. https://doi.org/10.1007/s11258-016-0649-y

Winkler, D. E., Gremer, J. R., Chapin, K. J., Kao, M., \& Huxman, T. E. (2018). Rapid alignment of functional trait variation with locality across the invaded range of Sahara mustard (Brassica tournefortii). American Journal of Botany, 105(7), 1188-1197. https://doi.org/10.1002/ajb2.1126

Winkler, D. E., Lin, M. Y., Delgadillo, J., Chapin, K. J., \& Huxman, T. E. (2019). Early life history responses and phenotypic shifts in a rare endemic plant responding to climate change. Conservation Physiology, 7. https://doi.org/10.1093/conphys/coz076

Wirth, C., \& Lichstein, J. W. (2009). The imprint of succession on oldgrowth forest carbon balances insights from a trait-based model of forest dynamics. In C. Wirth, G. Gleixner, \& M. Heimann (Eds.), Oldgrowth forests: Function, fate and value. Ecological Studies (Vol. 207). New York, NY; Berlin; Heidelberg: Springer.

Wright, I. J., Ackerly, D. D., Bongers, F., Harms, K. E., Ibarra-Manriquez, G., Martinez-Ramos, M., ... Wright, S. J. (2006). Relationships among ecologically important dimensions of plant trait variation in seven Neotropical forests. Annals of Botany, 99(5), 1003-1015. https://doi. org/10.1093/aob/mcl066

Wright, I. J., Cooke, J., Cernusak, L. A., Hutley, L. B., Scalon, M. C., Tozer, W. C., \& Lehmann, C. E. R. (2018). Stem diameter growth rates in a fireprone savanna correlate with photosynthetic rate and branch-scale 
biomass allocation, but not specific leaf area. Austral Ecology, 44(2), 339-350. https://doi.org/10.1111/aec.12678

Wright, J. P., \& Sutton-Grier, A. (2012). Does the leaf economic spectrum hold within local species pools across varying environmental conditions?Functional Ecology, 26(6), 1390-1398. https://doi. org/10.1111/1365-2435.12001

Wright, S. J., Kitajima, K., Kraft, N., Reich, P., Wright, I., Bunker, D. ... Zanne, A. (2010). Functional traits and the growth-mortality tradeoff in tropical trees. Ecology, 100514035422098. https://doi. org/10.1890/09-2335

Yguel, B., Bailey, R., Tosh, N. D., Vialatte, A., Vasseur, C., Vitrac, X., ... Prinzing, A. (2011). Phytophagy on phylogenetically isolated trees: Why hosts should escape their relatives. Ecology Letters, 14(11), 1117-1124. https://doi.org/10.1111/j.1461-0248.2011.01680.x

Yu, Q., Elser, J. J., He, N., Wu, H., Chen, Q., Zhang, G., \& Han, X. (2011). Stoichiometric homeostasis of vascular plants in the Inner Mongolia grassland. Oecologia, 166(1), 1-10. https://doi.org/10.1007/ s00442-010-1902-z

Zanne, A. E., Westoby, M., Falster, D. S., Ackerly, D. D., Loarie, S. R., Arnold, S. E. J., \& Coomes, D. A. (2010). Angiosperm wood structure: Global patterns in vessel anatomy and their relation to wood density and potential conductivity. American Journal of Botany, 97(2), 207-215. https://doi.org/10.3732/ajb.0900178

Zapata-Cuartas, M., Sierra, C. A., \& Alleman, L. (2012). Probability distribution of allometric coefficients and Bayesian estimation of aboveground tree biomass. Forest Ecology and Management, 277, 173-179. https://doi.org/10.1016/j.foreco.2012.04.030

Zheng, J., \& Martínez-Cabrera, H. I. (2013). Wood anatomical correlates with theoretical conductivity and wood density across China: Evolutionary evidence of the functional differentiation of axial and radial parenchyma. Annals of Botany, 112(5), 927-935. https://doi. org/10.1093/aob/mct153

Zheng, J., Zang, H., Yin, S., Sun, N., Zhu, P., Han, Y., ... Liu, C. (2018). Modeling height-diameter relationship for artificial monoculture Metasequoia glyptostroboides in sub-tropic coastal megacity Shanghai, China. Urban Forestry \& Urban Greening, 34, 226-232. https://doi.org/10.1016/j.ufug.2018.06.006

Zheng, W. (1983). Silva Sinica: Volume 1-4. Beijing: China Forestry Publishing House.

Ziemińska, K., Butler, D. W., Gleason, S. M., Wright, I. J., \& Westoby, M. (2013). Fibre wall and lumen fractions drive wood density variation across 24 Australian angiosperms. AoB PLANTS, 5. https://doi. org/10.1093/aobpla/plt046

Ziemińska, K., Westoby, M., \& Wright, I. J. (2015). Broad anatomical variation within a narrow wood density range-A study of twig wood across 69 Australian angiosperms. PLoS ONE, 10, e0124892. https://doi.org/10.1371/journal.pone.0124892

Zirbel, C. R., Bassett, T., Grman, E., \& Brudvig, L. A. (2017). Plant functional traits and environmental conditions shape community assembly and ecosystem functioning during restoration. Journal of Applied Ecology, 54(4), 1070-1079. https://doi.org/10.1111/13652664.12885

\section{SUPPORTING INFORMATION}

Additional supporting information may be found online in the Supporting Information section.

How to cite this article: Kattge J, Bönisch G, Díaz S, et al. TRY plant trait database - enhanced coverage and open access. Glob Change Biol. 2020;26:119-188. https://doi.org/10.1111/ gcb.14904

\section{APPENDIX}

Jens Kattge ${ }^{1,2}$, Gerhard Bönisch ${ }^{1}$, Sandra Díaz ${ }^{3}$, Sandra Lavorel ${ }^{4}$, lain Colin Prentice ${ }^{5}$, Paul Leadley ${ }^{6}$, Susanne Tautenhahn ${ }^{1}$, Gijsbert D. A. Werner ${ }^{7,8}$, Tuomas Aakala ${ }^{9}$, Mehdi Abedi ${ }^{10}$, Alicia T. R. Acosta ${ }^{11}$, George C. Adamidis ${ }^{12,13}$, Kairi Adamson ${ }^{14}$, Masahiro Aiba ${ }^{15}$, Cécile H. Albert ${ }^{16}$, Julio M. Alcántara ${ }^{17}$, Carolina Alcázar $C^{18}$, Izabela Aleixo ${ }^{19}$, Hamada $\mathrm{Ali}^{20}$, Bernard Amiaud ${ }^{21}$, Christian Ammer ${ }^{22,23}$, Mariano M. Amoroso ${ }^{24,25}$, Madhur Anand ${ }^{26}$, Carolyn Anderson ${ }^{27,28}$, Niels Anten ${ }^{29}$, Joseph Antos ${ }^{30}$, Deborah Mattos Guimarães Apgaua ${ }^{31}$, Tia-Lynn Ashman ${ }^{32}$, Degi Harja Asmara ${ }^{33}$, Gregory P. Asner ${ }^{34}$, Michael Aspinwall ${ }^{35}$, Owen Atkin ${ }^{36}$, Isabelle Aubin ${ }^{37}$, Lars BaastrupSpohr $^{38}$, Khadijeh Bahalkeh ${ }^{10}$, Michael Bahn ${ }^{39}$, Timothy Baker ${ }^{40}$, William J. Baker ${ }^{41}$, Jan P. Bakker ${ }^{42}$, Dennis Baldocchi ${ }^{43}$, Jennifer Baltzer ${ }^{44}$, Arindam Banerjee ${ }^{45}$, Anne Baranger ${ }^{46}$, Jos Barlow ${ }^{47}$, Diego R. Barneche ${ }^{48}$, Zdravko Baruch ${ }^{49}$, Denis Bastianelli ${ }^{50,51}$, John Battles ${ }^{52}$, William Bauerle ${ }^{53}$, Marijn Bauters ${ }^{54,55}$, Erika Bazzato ${ }^{56}$, Michael Beckmann ${ }^{57}$, Hans Beeckman ${ }^{58}$, Carl Beierkuhnlein ${ }^{59}$, Renee Bekker ${ }^{60}$, Gavin Belfry ${ }^{61,62}$, Michael Belluau ${ }^{63}$, Mirela Beloiu ${ }^{64}$, Raquel Benavides ${ }^{65}$, Lahcen Benomar ${ }^{66}$, Mary Lee BerdugoLattke $^{67,68}$, Erika Berenguer ${ }^{69}$, Rodrigo Bergamin $^{70}$, Joana Bergmann ${ }^{71,72}$, Marcos Bergmann Carlucci ${ }^{73}$, Logan Berner ${ }^{74}$, Markus Bernhardt-Römermann ${ }^{75}$, Christof Bigler $^{76}$, Anne D. Bjorkman ${ }^{77}$, Chris Blackman ${ }^{78}$, Carolina Blanco ${ }^{79}$, Benjamin Blonder ${ }^{80,62}$, Dana Blumenthal ${ }^{81}$, Kelly T. BocanegraGonzález $^{82}$, Pascal Boeckx ${ }^{83}$, Stephanie Bohlman ${ }^{84}$, Katrin BöhningGaese $^{85,86}$, Laura Boisvert-Marsh ${ }^{37}$, William Bond ${ }^{87,88}$, Ben Bond-Lamberty ${ }^{89}$, Arnoud Boom ${ }^{90}$, Coline C. F. Boonman ${ }^{91}$, Kauane Bordin ${ }^{92}$, Elizabeth H. Boughton ${ }^{93}$, Vanessa Boukili ${ }^{94}$, David M. J. S. Bowman ${ }^{95}$, Sandra Bravo ${ }^{96}$, Marco Richard Brendel ${ }^{97}$, Martin R. Broadley ${ }^{98}$, Kerry A. Brown 99 , Helge Bruelheide ${ }^{100,2}$, Federico Brumnich ${ }^{25,101}$, Hans Henrik Bruun ${ }^{38}$, David Bruy ${ }^{102,103}$, Serra W. Buchanan ${ }^{104}$, Solveig Franziska Bucher ${ }^{105}$, Nina Buchmann ${ }^{76}$, Robert Buitenwerf ${ }^{106,107}$, Daniel E. Bunker ${ }^{108}$, Jana Bürger ${ }^{109}$, Sabina Burrascano ${ }^{110}$, David F. R. P. Burslem ${ }^{111}$, Bradley J. Butterfield ${ }^{112}$, Chaeho Byun ${ }^{113}$, Marcia Marques ${ }^{114}$, Marina C. Scalon ${ }^{115}$, Marco Caccianiga ${ }^{116}$, Marc Cadotte ${ }^{104}$, Maxime Cailleret ${ }^{117,118,119}$, James Camac ${ }^{120}$, Jesús Julio Camarero ${ }^{121}$, Courtney Campany ${ }^{122}$, Giandiego Campetella ${ }^{123}$, Juan Antonio Campos ${ }^{124}$, Laura Cano-Arboleda ${ }^{125,68}$, Roberto Canullo ${ }^{123}$, Michele Carbognani ${ }^{126}$, Fabio Carvalho ${ }^{47}$, Fernando Casanoves ${ }^{127}$, Bastien Castagneyrol ${ }^{128}$, Jane A. Catford ${ }^{129}$, Jeannine CavenderBares $^{130}$, Bruno E. L. Cerabolini ${ }^{131}$, Marco Cervellini ${ }^{123,132}$, Eduardo Chacón-Madrigal ${ }^{133}$, Kenneth Chapin $^{134}$, F. Stuart Chapin $^{135}$, Stefano Chelli ${ }^{123}$, Si-Chong Chen ${ }^{136}$, Anping Chen ${ }^{137}$, Paolo Cherubini ${ }^{138,139}$, Francesco Chianucci ${ }^{140}$, Brendan Choat ${ }^{141}$, Kyong-Sook Chung ${ }^{142}$, Milan Chytrý ${ }^{143}$, Daniela Ciccarelli ${ }^{144}$, Lluís Coll ${ }^{145,146}$, Courtney G. Collins ${ }^{147}$, Luisa Conti ${ }^{148,149}$, David Coomes ${ }^{150}$, Johannes H. C. Cornelissen ${ }^{151}$, William K. Cornwell ${ }^{152}$, Piermaria Corona ${ }^{140}$, Marie Coyea ${ }^{153}$, Joseph Craine ${ }^{154}$, Dylan Craven ${ }^{155}$, Joris P. G. M. Cromsigt ${ }^{156,157}$, Anikó Csecserits ${ }^{158}$, Katarina Cufar ${ }^{159}$, Matthias Cuntz ${ }^{160}$, Ana 
Carolina da Silva ${ }^{161}$, Kyla M. Dahlin ${ }^{162}$, Matteo Dainese ${ }^{163}$, Igor Dalke ${ }^{164}$, Michele Dalle Fratte ${ }^{131}$, Anh Tuan Dang-Le ${ }^{165}$, Jirí Danihelka ${ }^{143,149}$, Masako Dannoura ${ }^{166,167}$, Samantha Dawson ${ }^{168}$, Arend Jacobus de Beer ${ }^{169}$, Angel De Frutos ${ }^{2,57}$, Jonathan R. De Long $^{170}$, Benjamin Dechant ${ }^{171,172,173}$, Sylvain Delagrange ${ }^{174,175}$, Nicolas Delpierre ${ }^{6}$, Géraldine Derroire ${ }^{176}$, Arildo S. Dias ${ }^{177}$, Milton Hugo Diaz-Toribio ${ }^{178}$, Panayiotis G. Dimitrakopoulos ${ }^{12}$, Mark Dobrowolski ${ }^{179,180}$, Daniel Doktor ${ }^{57}$, Pavel Dřevojan ${ }^{143}$, Ning Dong ${ }^{181}$, John Dransfield ${ }^{41}$, Stefan Dressler ${ }^{182}$, Leandro Duarte ${ }^{79}$, Emilie Ducouret ${ }^{176}$, Stefan Dullinger ${ }^{183}$, Walter Durka ${ }^{2,184}$, Remko Duursma ${ }^{141}$, Olga Dymova ${ }^{164}$, Anna E-Vojtkó ${ }^{149,185}$, Rolf Lutz Eckstein ${ }^{186}$, Hamid Ejtehadi ${ }^{187}$, James Elser ${ }^{188,189}$, Thaise Emilio ${ }^{190}$, Kristine Engemann ${ }^{106}$, Mohammad Bagher Erfanian ${ }^{187}$, Alexandra Erfmeier ${ }^{2,191}$, Adriane Esquivel-Muelbert ${ }^{40,192}$, Gerd Esser ${ }^{193}$, Marc Estiarte ${ }^{194,195}$, Tomas F. Domingues ${ }^{196}$, William F. Fagan ${ }^{197}$, Jaime Fagúndez ${ }^{198}$, Daniel S. Falster ${ }^{199}$, Ying Fan ${ }^{200}$, Jingyun Fang ${ }^{201}$, Emmanuele Farris ${ }^{202}$, Fatih Fazlioglu ${ }^{203}$, Yanhao Feng ${ }^{204}$, Fernando FernandezMendez $^{82,205}$, Carlotta Ferrara ${ }^{140}$, Joice Ferreira ${ }^{206}$, Alessandra Fidelis ${ }^{207}$, Bryan Finegan ${ }^{127}$, Jennifer Firn ${ }^{208}$, Timothy J. Flowers ${ }^{209}$, Dan F. B. Flynn ${ }^{210}$, Veronika Fontana ${ }^{163}$, Estelle Forey ${ }^{211}$, Cristiane Forgiarini ${ }^{212}$, Louis François ${ }^{213}$, Marcelo Frangipani ${ }^{26,79}$, Dorothea Frank ${ }^{1}$, Cedric FrenetteDussault $^{214}$, Grégoire T. Freschet ${ }^{215}$, Ellen L. Fry ${ }^{216}$, Nikolaos M. Fyllas ${ }^{12}$, Guilherme G. Mazzochini ${ }^{217}$, Sophie Gachet ${ }^{16}$, Rachael Gallagher ${ }^{181}$, Gislene Ganade ${ }^{218}$, Francesca Ganga ${ }^{56}$, Pablo García-Palacios ${ }^{219}$, Veronica Gargaglione ${ }^{220}$, Eric Garnier ${ }^{221}$, Jose Luis Garrido ${ }^{222,223}$, André Luís de Gasper ${ }^{224}$, Guillermo GeaIzquierdo ${ }^{225}$, David Gibson ${ }^{226}$, Andrew N. Gillison ${ }^{227}$, Aelton Giroldo ${ }^{228}$, Mary-Claire Glasenhardt ${ }^{229}$, Sean Gleason ${ }^{230}$, Mariana Gliesch ${ }^{231}$, Emma Goldberg ${ }^{232}$, Bastian Göldel ${ }^{106}$, Erika Gonzalez-Akre ${ }^{233}$, Jose L. Gonzalez-Andujar ${ }^{234}$, Andrés González-Melo ${ }^{235}$, Ana González-Robles ${ }^{236}$, Bente Jessen Graae ${ }^{237}$, Elena Granda ${ }^{238}$, Sarah Graves ${ }^{239}$, Walton A. Green ${ }^{240}$, Thomas Gregor ${ }^{182}$, Nicolas Gross ${ }^{241,242}$, Greg R. Guerin ${ }^{49}$, Angela Günther ${ }^{1}$, Alvaro G. Gutiérrez ${ }^{243}$, Lillie Haddock ${ }^{244}$, Anna Haines ${ }^{245}$, Jefferson Hall ${ }^{246}$, Alain Hambuckers ${ }^{247}$, Wenxuan Han ${ }^{248,249,250}$, Sandy P. Harrison ${ }^{251}$, Wesley Hattingh ${ }^{252}$, Joseph E. Hawes ${ }^{253,254}$, Tianhua He $\mathrm{He}^{255,256}$, Pengcheng $\mathrm{He}^{257}$, Jacob Mason Heberling ${ }^{258}$, Aveliina Helm ${ }^{259}$, Stefan Hempel ${ }^{71,72}$, Jörn Hentschel ${ }^{260}$, Bruno Hérault ${ }^{261,262}$, AnaMaria Hereş ${ }^{263,264}$, Katharina Herz ${ }^{100}$, Myriam Heuertz ${ }^{128}$, Thomas Hickler ${ }^{85,265}$, Peter Hietz ${ }^{266}$, Pedro Higuchi ${ }^{161}$, Andrew L. Hipp 229,267 , Andrew Hirons ${ }^{268}$, Maria Hock ${ }^{191}$, James Aaron Hogan ${ }^{269,270}$, Karen Holl ${ }^{271}$, Olivier Honnay ${ }^{272,273}$, Daniel Hornstein ${ }^{59}$, Enqing Hou ${ }^{257}$, Nate Hough-Snee ${ }^{274}$, Knut Anders Hovstad ${ }^{275}$, Tomoaki Ichie ${ }^{276}$, Boris Igić ${ }^{277}$, Estela Illa ${ }^{278}$, Marney Isaac ${ }^{279}$, Masae Ishihara ${ }^{280}$, Leonid Ivanov ${ }^{281,282}$, Larissa Ivanova ${ }^{281,282}$, Colleen M. Iversen ${ }^{283}$, Jordi Izquierdo ${ }^{284}$, Robert B. Jackson ${ }^{285}$, Benjamin Jackson ${ }^{286}$, Hervé Jactel ${ }^{128}$, Andrzej M. Jagodzinski ${ }^{287,288}$, Ute Jandt ${ }^{2,289}$, Steven Jansen ${ }^{290}$, Thomas Jenkins ${ }^{7,62}$, Anke Jentsch ${ }^{291}$, Jens Rasmus Plantener Jespersen ${ }^{292}$, Guo-Feng Jiang ${ }^{293,294}$, Jesper
Liengaard Johansen ${ }^{295}$, David Johnson ${ }^{245}$, Eric J. Jokela ${ }^{84}$, Carlos Alfredo Joly ${ }^{296}$, Gregory J. Jordan ${ }^{297}$, Grant Stuart Joseph ${ }^{298,299}$, Decky Junaedi ${ }^{300,301}$, Robert R. Junker ${ }^{302,303}$, Eric Justes ${ }^{304}$, Richard Kabzems ${ }^{305}$, Jeffrey Kane ${ }^{306}$, Zdenek Kaplan ${ }^{307,308,}$ Teja Kattenborn ${ }^{309}$, Lyudmila Kavelenova ${ }^{310}$, Elizabeth Kearsley ${ }^{311}$, Anne Kempel ${ }^{312}$, Tanaka Kenzo ${ }^{313}$, Andrew Kerkhoff ${ }^{314}$, Mohammed I. Khali ${ }^{315,316}$, Nicole L. Kinlock ${ }^{317}$, Wilm Daniel Kissling ${ }^{318}$, Kaoru Kitajima ${ }^{166,239,255}$, Thomas Kitzberger ${ }^{319,320}$, Rasmus Kjøller ${ }^{38}$, Tamir Klein ${ }^{321}$, Michael Kleyer ${ }^{322}$, Jitka Klimešová ${ }^{149,323}$, Joice Klipel ${ }^{324}$, Brian Kloeppel ${ }^{325}$, Stefan Klotz ${ }^{2,326}$, Johannes M. H. Knops ${ }^{327}$, Takashi Kohyama ${ }^{328}$, Fumito Koike ${ }^{329}$, Johannes Kollmann ${ }^{330}$, Benjamin Komac ${ }^{331}$, Kimberly Komatsu ${ }^{332}$, Christian König ${ }^{333,334}$, Nathan J. B. Kraft ${ }^{335}$, Koen Kramer ${ }^{336,337}$, Holger Kreft ${ }^{334,338}$, Ingolf Kühn ${ }^{2,184,339}$, Dushan Kumarathunge ${ }^{141,340}$, Jonas Kuppler ${ }^{341}$, Hiroko Kurokawa ${ }^{313}$, Yoko Kurosawa ${ }^{342}$, Shem Kuyah ${ }^{343}$, Jean-Paul Laclau ${ }^{344,345}$, Benoit Lafleur ${ }^{346}$, Erik Lallai ${ }^{56}$, Eric Lamb ${ }^{347}$, Andrea Lamprecht ${ }^{348}$, Daniel J. Larkin ${ }^{349}$, Daniel Laughlin ${ }^{350}$, Yoann Le Bagousse-Pinguet ${ }^{351}$, Guerric le Maire ${ }^{344,345}$, Peter C. le Roux ${ }^{169}$, Elizabeth le Roux ${ }^{352}$, Tali Lee ${ }^{353}$, Frederic Lens ${ }^{354}$, Simon L. Lewis ${ }^{40,355}$, Barbara Lhotsky ${ }^{154}$, Yuanzhi Li ${ }^{356}$, Xine Li ${ }^{357}$, Jeremy W. Lichstein ${ }^{178}$, Mario Liebergesell ${ }^{358}$, Jun Ying Lim $^{318}$, Yan-Shih $\operatorname{Lin}^{359}$, Juan Carlos Linares ${ }^{360}$, Chunjiang $\mathrm{Liu}^{361,362}$, Daijun $\mathrm{Liu}^{192}$, Udayangani $\mathrm{Liu}^{136}$, Stuart Livingstone ${ }^{104}$, Joan Llusià ${ }^{363}$, Madelon Lohbeck ${ }^{364,365}$, Álvaro López-García ${ }^{38,366}$, Gabriela Lopez-Gonzalez ${ }^{367}$, Zdeňka Lososová ${ }^{143}$, Frédérique Louault ${ }^{241}$, Balázs A. Lukács ${ }^{368}$, Petr Lukeš ${ }^{369}$, Yunjian Luo ${ }^{370,371}$, Michele Lussu ${ }^{56}$, Siyan $\mathrm{Ma}^{52}$, Camilla Maciel Rabelo Pereira ${ }^{372}$, Michelle Mack ${ }^{112}$, Vincent Maire ${ }^{373}$, Annikki Mäkelä ${ }^{374}$, Harri Mäkinen ${ }^{375}$, Ana Claudia Mendes Malhado ${ }^{376}$, Azim Mallik ${ }^{377}$, Peter Manning ${ }^{85}$, Stefano Manzoni ${ }^{378,379}$, Zuleica Marchetti ${ }^{25,101}$, Luca Marchino ${ }^{140}$, Vinicius Marcilio-Silva ${ }^{380}$, Eric Marcon ${ }^{176}$, Michela Marignani ${ }^{56}$, Lars Markesteijn ${ }^{381}$, Adam Martin ${ }^{382}$, Cristina Martínez-Garza ${ }^{383}$, Jordi Martínez-Vilalta ${ }^{195,384}$, Tereza Mašková ${ }^{323}$, Kelly Mason ${ }^{385}$, Norman Mason ${ }^{386}$, Tara Joy Massad ${ }^{387}$, Jacynthe Masse ${ }^{388,389}$, Itay Mayrose ${ }^{390}$, James McCarthy ${ }^{391,392,393}$, M. Luke McCormack ${ }^{394}$, Katherine McCulloh ${ }^{395}$, Ian R. McFadden ${ }^{118,119,335}$, Brian J. McGill ${ }^{396}$, Mara Y. McPartland ${ }^{397}$, Juliana S. Medeiros ${ }^{398}$, Belinda Medlyn ${ }^{141}$, Pierre Meerts ${ }^{399}$, Zia Mehrabi ${ }^{400}$, Patrick Meir ${ }^{401,402}$, Felipe P. L. Melo ${ }^{403}$, Maurizio Mencuccini ${ }^{404,405}$, Céline Meredieu ${ }^{406}$, Julie Messier ${ }^{407}$, Ilona Mészáros ${ }^{408}$, Juha Metsaranta ${ }^{409}$, Sean T. Michaletz ${ }^{410}$, Chrysanthi Michelaki ${ }^{12}$, Svetlana Migalina ${ }^{281,282}$, Ruben Milla ${ }^{411}$, Jesse E. D. Miller ${ }^{412}$, Vanessa Minden ${ }^{413,414}$, Ray Ming ${ }^{415}$, Karel Mokany ${ }^{416}$, Angela T. Moles ${ }^{199}$, Attila Molnár V ${ }^{417}$, Jane Molofsky ${ }^{418}$, Martin Molz ${ }^{419}$, Rebecca A. Montgomery ${ }^{420}$, Arnaud Monty ${ }^{421}$, Lenka Moravcová ${ }^{422}$, Alvaro Moreno-Martínez ${ }^{423}$, Marco Moretti ${ }^{119}$, Akira S. Mori ${ }^{329}$, Shigeta Mori ${ }^{342}$, Dave Morris ${ }^{424}$, Jane Morrison ${ }^{425}$, Ladislav Mucina ${ }^{426,427}$, Sandra Mueller ${ }^{428}$, Christopher D. Muir ${ }^{429}$, Sandra Cristina Müller ${ }^{430}$, François Munoz ${ }^{431,432}$, Isla H. Myers-Smith ${ }^{402}$, Randall W. Myster ${ }^{433}$, Masahiro Nagano ${ }^{434}$, Shawna Naidu ${ }^{415}$, Ayyappan Narayanan ${ }^{435}$, Balachandran Natesan ${ }^{435}$, Luka Negoita ${ }^{436}$, Andrew S. Nelson ${ }^{437}$, Eike Lena Neuschulz ${ }^{85}$, Jian $\mathrm{Ni}^{438}$, Georg Niedrist ${ }^{163}$, 
Jhon Nieto ${ }^{439,440}$, Ülo Niinemets ${ }^{441}$, Rachael Nolan ${ }^{141}$, Henning Nottebrock ${ }^{442}$, Yann Nouvellon ${ }^{344,345}$, Alexander Novakovskiy ${ }^{164}$, The Nutrient Network, Kristin Odden Nystuen ${ }^{443,444}$, Anthony O'Grady ${ }^{445}$, Kevin O'Hara ${ }^{52}$, Andrew O'Reilly-Nugent ${ }^{446}$, Simon Oakley ${ }^{385}$, Walter Oberhuber ${ }^{447}$, Toshiyuki Ohtsuka $^{448}$, Ricardo Oliveira ${ }^{449}$, Kinga Öllerer ${ }^{450,451}$, Mark E. Olson ${ }^{452,453}$, Vladimir Onipchenko ${ }^{454}$, Yusuke Onoda ${ }^{455}$, Renske E. Onstein ${ }^{2}$, Jenny C. Ordonez ${ }^{456}$, Noriyuki Osada ${ }^{457}$, Ivika Ostonen ${ }^{259}$, Gianluigi Ottaviani ${ }^{149}$, Sarah Otto ${ }^{458}$, Gerhard E. Overbeck ${ }^{79}$, Wim A. Ozinga ${ }^{459}$, Anna T. Pahl ${ }^{460}$, C. E. Timothy Paine ${ }^{461}$, Robin J. Pakeman ${ }^{462}$, Aristotelis C. Papageorgiou ${ }^{463}$, Evgeniya Parfionova ${ }^{310}$, Meelis Pärtel ${ }^{464}$, Marco Patacca ${ }^{336}$, Susana Paula ${ }^{465}$, Juraj Paule ${ }^{182}$, Harald Pauli ${ }^{348}$, Juli G. Pausas ${ }^{466}$, Begoña Peco ${ }^{467}$, Josep Penuelas ${ }^{195,468}$, Antonio Perea ${ }^{469}$, Pablo Luis Peri ${ }^{470,471}$, Ana Carolina PetiscoSouza ${ }^{472}$, Alessandro Petraglia ${ }^{126}$, Any Mary Petritan ${ }^{473}$, Oliver L. Phillips ${ }^{40}$, Simon Pierce ${ }^{474}$, Valério D. Pillar ${ }^{475}$, Jan Pisek ${ }^{14}$, Alexandr Pomogaybin ${ }^{476}$, Hendrik Poorter ${ }^{181,477}$, Angelika Portsmuth ${ }^{478}$, Peter Poschlod ${ }^{479}$, Catherine Potvin ${ }^{480}$, Devon Pounds ${ }^{481}$, A. Shafer Powell ${ }^{482}$, Sally A. Power ${ }^{141}$, Andreas Prinzing ${ }^{483}$, Giacomo Puglielli ${ }^{441}$, Petr Pyšek ${ }^{422,484}$, Valerie Raevel ${ }^{102,432,485}$, Anja Rammig ${ }^{486}$, Johannes Ransijn ${ }^{372}$, Courtenay A. Ray ${ }^{62,80}$, Peter B. Reich ${ }^{45,141}$, Markus Reichstein ${ }^{1}$, Douglas E. B. Reid ${ }^{424}$, Maxime Réjou-Méchain ${ }^{102}$, Victor Resco de Dios $^{487,488}$, Sabina Ribeiro ${ }^{489}$, Sarah Richardson ${ }^{490}$, Kersti Riibak ${ }^{464}$, Matthias C. Rillig ${ }^{72,491}$, Fiamma Riviera ${ }^{492}$, Elisabeth M. R. Robert ${ }^{493,494,495}$, Scott Roberts ${ }^{496}$, Bjorn Robroek $^{497,498}$, Adam Roddy ${ }^{499}$, Arthur Vinicius Rodrigues ${ }^{500}$, Alistair Rogers ${ }^{501}$, Emily Rollinson ${ }^{502}$, Victor Rolo ${ }^{503}$, Christine Römermann ${ }^{2,75}$, Dina Ronzhina ${ }^{281,282}$, Christiane Roscher ${ }^{2,504}$, Julieta A. Rosell ${ }^{505}$, Milena Fermina Rosenfield ${ }^{506}$, Christian Rossi ${ }^{507,508,509}$, David B. Roy ${ }^{510}$, Samuel Royer-Tardif ${ }^{511}$, Nadja Rüger ${ }^{2,246}$, Ricardo RuizPeinado ${ }^{512,513}$, Sabine B. Rumpf ${ }^{183,514}$, Graciela M. Rusch ${ }^{515}$, Masahiro Ryo ${ }^{72,491}$, Lawren Sack ${ }^{335}$, Angela Saldaña ${ }^{453}$, Beatriz Salgado-Negret ${ }^{516}$, Roberto Salguero-Gomez ${ }^{517}$, Ignacio SantaRegina $^{518}$, Ana Carolina Santacruz-García ${ }^{25,96}$, Joaquim Santos ${ }^{519}$, Jordi Sardans ${ }^{468}$, Brandon Schamp ${ }^{520}$, Michael Scherer-Lorenzen ${ }^{428}$, Matthias Schleuning ${ }^{85}$, Bernhard Schmid ${ }^{521}$, Marco Schmidt ${ }^{522,523}$, Sylvain Schmitt ${ }^{128}$, Julio V. Schneider ${ }^{182,524}$, Simon D. Schowanek ${ }^{106,107}$, Julian Schrader ${ }^{334}$, Franziska Schrodt ${ }^{98}$, Bernhard Schuldt ${ }^{525}$, Frank Schurr ${ }^{97}$, Galia Selaya Garvizu ${ }^{526}$, Marina Semchenko ${ }^{527}$, Colleen Seymour ${ }^{528}$, Julia C. Sfair ${ }^{529}$, Joanne M. Sharpe ${ }^{530}$, Christine S. Sheppard ${ }^{97}$, Serge Sheremetiev ${ }^{531}$, Satomi Shiodera ${ }^{532,533}$, Bill Shipley ${ }^{534}$, Tanvir Ahmed Shovon ${ }^{535}$, Alrun Siebenkäs ${ }^{536}$, Carlos Sierra ${ }^{1}$, Vasco Silva ${ }^{537}$, Mateus Silva ${ }^{538}$, Tommaso Sitzia ${ }^{539}$, Henrik Sjöman ${ }^{540,541,542}$, Martijn Slot ${ }^{246}$, Nicholas G. Smith ${ }^{543}$, Darwin Sodhi ${ }^{544}$, Pamela Soltis ${ }^{545}$, Douglas Soltis ${ }^{545}$, Ben Somers ${ }^{546}$, Grégory Sonnier ${ }^{547}$, Mia Vedel Sørensen ${ }^{237}$, Enio Egon Sosinski $\mathrm{Jr}^{548}$, Nadejda A. Soudzilovskaia ${ }^{549}$, Alexandre F. Souza ${ }^{550}$, Marko Spasojevic ${ }^{551}$, Marta Gaia Sperandii ${ }^{11}$, Amanda B. $\operatorname{Stan}^{552}$, James Stegen ${ }^{27}$, Klaus Steinbauer ${ }^{348}$, Jörg G. Stephan ${ }^{168,553}$, Frank Sterck ${ }^{554}$, Dejan B. Stojanovic ${ }^{555}$, Tanya Strydom ${ }^{556}$, Maria Laura Suarez ${ }^{557}$, Jens-Christian Svenning ${ }^{107,106}$,
Ivana Svitková ${ }^{558}$, Marek Svitok ${ }^{559,560}$, Miroslav Svoboda ${ }^{561}$, Emily Swaine ${ }^{111}$, Nathan Swenson ${ }^{562}$, Marcelo Tabarelli ${ }^{563}$, Kentaro Takagi ${ }^{564}$, Ulrike Tappeiner ${ }^{39,163}$, Rubén Tarifa ${ }^{565}$, Simon Tauugourdeau ${ }^{51,566}$, Cagatay Tavsanoglu ${ }^{567}$, Mariska te Beest ${ }^{568,569}$, Leho Tedersoo ${ }^{259}$, Nelson Thiffault ${ }^{570}$, Dominik Thom ${ }^{571}$, Evert Thomas ${ }^{572}$, Ken Thompson ${ }^{573}$, Peter E. Thornton ${ }^{283}$, Wilfried Thuiller ${ }^{4}$, Lubomír Tichý ${ }^{143}$, David Tissue ${ }^{141}$, Mark G. Tjoelker ${ }^{141}$, David Yue Phin Tng ${ }^{574}$, Joseph Tobias ${ }^{575}$, Péter Török ${ }^{576,577}$, Tonantzin Tarin ${ }^{578}$, José M. Torres-Ruiz ${ }^{579}$, Béla Tóthmérész ${ }^{580}$, Martina Treurnicht ${ }^{88,581}$, Valeria Trivellone ${ }^{582}$, Franck Trolliet ${ }^{583}$, Volodymyr Trotsiuk ${ }^{119,561,584}$, James L. Tsakalos ${ }^{585}$, loannis Tsiripidis ${ }^{586}$, Niklas Tysklind ${ }^{587}$, Toru Umehara ${ }^{588}$, Vladimir Usoltsev ${ }^{589,590}$, Matthew Vadeboncoeur ${ }^{591}$, Jamil Vaezi ${ }^{592}$, Fernando Valladares ${ }^{65}$, Jana Vamosi ${ }^{593}$, Peter M. van Bodegom ${ }^{549}$, Michiel van Breugel ${ }^{594,595,596}$, Elisa Van Cleemput ${ }^{546}$, Martine van de Weg ${ }^{597}$, Stephni van der Merwe ${ }^{87}$, Fons van der Plas ${ }^{598}$, Masha T. van der Sande ${ }^{318,364,599}$, Mark van Kleunen ${ }^{600,601}$, Koenraad Van Meerbeek $^{546}$, Mark Vanderwel ${ }^{535}$, Kim André Vanselow ${ }^{602}$, Angelica Vårhammar ${ }^{141}$, Laura Varone ${ }^{603}$, Maribel Yesenia Vasquez Valderrama ${ }^{440,604}$, Kiril Vassilev ${ }^{605}$, Mark Vellend ${ }^{534}$, Erik J. Veneklaas ${ }^{606}$, Hans Verbeeck ${ }^{311}$, Kris Verheyen ${ }^{607}$, Alexander Vibrans ${ }^{224}$, Ima Vieira ${ }^{608}$, Jaime Villacís ${ }^{609}$, Cyrille Violle ${ }^{221}$, Pandi Vivek ${ }^{610,611}$, Katrin Wagner ${ }^{612}$, Matthew Waldram ${ }^{90}$, Anthony Waldron ${ }^{613,614}$, Anthony P. Walker ${ }^{482}$, Martyn Waller ${ }^{99}$, Gabriel Walther ${ }^{75}$, Han Wang ${ }^{615}$, Feng Wang ${ }^{616}$, Weiqi Wang ${ }^{617}$, Harry Watkins ${ }^{618}$, James Watkins ${ }^{619}$, Ulrich Weber ${ }^{1}$, James T. Weedon ${ }^{620}$, Liping Wei ${ }^{346}$, Patrick Weigelt ${ }^{334}$, Evan Weiher ${ }^{353}$, Aidan W. Wells ${ }^{62,621}$, Camilla Wellstein ${ }^{622}$, Elizabeth Wenk ${ }^{199}$, Mark Westoby ${ }^{181}$, Alana Westwood ${ }^{623}$, Philip John White ${ }^{624,625}$, Mark Whitten ${ }^{239}$, Mathew Williams ${ }^{402}$, Daniel E. Winkler ${ }^{626,627}$, Klaus Winter ${ }^{246}$, Chevonne Womack ${ }^{169}$, Ian J. Wright ${ }^{181}$, S. Joseph Wright ${ }^{246}$, Justin Wright ${ }^{628}$, Bruno X. Pinho ${ }^{563}$, Fabiano Ximenes ${ }^{629}$, Toshihiro Yamada ${ }^{630}$, Keiko Yamaji ${ }^{631}$, Ruth Yanai ${ }^{632}$, Nikolay Yankov ${ }^{476}$, Benjamin Yguel ${ }^{633}$, Kátia Janaina Zanini ${ }^{634}$, Amy E. Zanne ${ }^{635}$, David Zelený ${ }^{636}$, YunPeng Zhao ${ }^{637}$, Jingming Zheng ${ }^{638}$, Ji Zheng ${ }^{361,362}$, Kasia Ziemińska ${ }^{5}$, Chad R. Zirbel ${ }^{130}$, Georg Zizka ${ }^{86,182}$, Irié Casimir Zo-Bi ${ }^{639}$, Gerhard Zotz ${ }^{246,640}$, Christian Wirth ${ }^{1,2,358}$

${ }^{1}$ Max Planck Institute for Biogeochemistry, Jena, Germany, ${ }^{2}$ German Center for Integrative Biodiversity Research (iDiv) Halle-JenaLeipzig, Leipzig, Germany, ${ }^{3}$ Consejo Nacional de Investigaciones Científicas y Técnicas, Instituto Multidisciplinario de Biología Vegetal (IMBIV), and Factulad de Ciencias Exactas, Físicas y Naturales, Universidad Nacional de Córdoba, Córdoba, Argentina, ${ }^{4}$ Univ. Grenoble Alpes, CNRS, Univ. Savoie Mont Blanc, LECA, Grenoble, France, ${ }^{5}$ Imperial College, London, UK, ${ }^{6}$ Ecologie Systématique Evolution, CNRS, AgroParisTech, University of Paris-Sud, Université Paris-Saclay, Orsay, France, ${ }^{7}$ Department of Zoology, University of Oxford, Oxford, UK, ${ }^{8}$ Balliol College, University of Oxford, Oxford, UK, ${ }^{9}$ University of Helsinki, Helsinki, Finland, ${ }^{10}$ Department of Range Management, Faculty of Natural Resources and Marine Sciences, Tarbiat Modares University, Noor, Iran, ${ }^{11}$ University of 
Roma Tre, Rome, Italy, ${ }^{12}$ Biodiversity Conservation Laboratory, Department of Environment, University of the Aegean, Mytilene, Greece, ${ }^{13}$ Institute of Ecology and Evolution, University of Bern, Bern, Switzerland, ${ }^{14}$ Tartu Observatory, University of Tartu, Tartumaa, Estonia, ${ }^{15}$ Graduate School of Life Sciences, Tohoku University, Sendai, Japan, ${ }^{16}$ Aix Marseille Univ, Univ Avignon, CNRS, IRD, IMBE, Marseille, France, ${ }^{17}$ Universidad de Jaén, Jaén, Spain, ${ }^{18}$ Instituto Alexander Von Humboldt, Bogota, Colombia, ${ }^{19}$ National Institute of Amazonian Research (INPA), Manaus, Brazil, ${ }^{20}$ Botany Department, Faculty of Science, Suez Canal University, Ismailia, Egypt, ${ }^{21}$ Université de Lorraine, Lorraine, France, ${ }^{22}$ Forest Sciences, University of Göttingen, Göttingen, Germany, ${ }^{23}$ Centre for Biodiversity and Sustainable Land-use, University of Göttingen, Göttingen, Germany, ${ }^{24}$ Instituto de Investigaciones en Recursos Naturales, Agroecología y Desarrollo Rural (IRNAD), Universidad Nacional de Río Negro, El Bolsón, Argentina, ${ }^{25}$ Conicet-Consejo Nacional de Investigaciones Científicas y Técnicas, Buenos Aires, Argentina, ${ }^{26}$ University of Guelph, Guelph, ON, Canada, ${ }^{27}$ Pacific Northwest National Laboratory, Richland, WA, USA, ${ }^{28}$ University of Massachusetts Amherst, Amherst, MA, USA, ${ }^{29}$ Centre for Crop Systems Analysis, Wageningen University, Wageningen, The Netherlands, ${ }^{30}$ University of Victoria, Victoria, BC, Canada, ${ }^{31}$ College of Science \& Engineering, James Cook University, Smithfield, Qld, Australia, ${ }^{32}$ University of Pittsburgh, Pittsburgh, PA, USA, ${ }^{33}$ Centre for Forest Research, Institute for Integrative Systems Biology, Université Laval, Quebec, QC, Canada, ${ }^{34}$ Arizona State University, Tempe, AZ, USA, ${ }^{35}$ Department of Biology, University of North Florida, Jacksonville, FL, USA, ${ }^{36}$ ARC Centre for Excellence in Plant Energy Biology, Australian National University, Acton, ACT, Australia, ${ }^{37}$ Great Lakes Forestry Centre, Canadian Forest Service, Natural Resources Canada, Sault Ste. Marie, ON, Canada, ${ }^{38}$ Department of Biology, University of Copenhagen, Copenhagen, Denmark, ${ }^{39}$ Department of Ecology, University of Innsbruck, Innsbruck, Austria, ${ }^{40}$ School of Geography, University of Leeds, Leeds, UK, ${ }^{41}$ Royal Botanic Gardens Kew, Richmond, UK, ${ }^{42}$ Conservation Ecology, Groningen Institute for Evolutionary Life Sciences (GELIFES), University of Groningen, Groningen, The Netherlands, ${ }^{43}$ Department of Environmental Science, Policy and Management, University of California Berkeley, Berkeley, CA, USA, ${ }^{44}$ Biology Department, Wilfrid Laurier University, Waterloo, ON, Canada, ${ }^{45}$ Department of Forest Resources, University of Minnesota, St. Paul, MN, USA, ${ }^{46}$ AgroParisTech, Paris, France, ${ }^{47}$ Lancaster Environment Centre, Lancaster University, Lancaster, UK, ${ }^{48}$ College of Life and Environmental Sciences, University of Exeter, Penryn, UK, ${ }^{49}$ School of Biological Sciences, The University of Adelaide, Adelaide, SA, Australia, ${ }^{50}$ CIRAD, UMR SELMET, Montpellier, France, ${ }^{51}$ SELMET, CIRAD, INRA, Univ Montpellier, Montpellier SupAgro, France, ${ }^{52}$ University of California at Berkeley, Berkeley, CA, USA, ${ }^{53}$ Department of Horticulture and Landscape Architecture, Colorado State University, Fort Collins, CO, USA, ${ }^{54}$ Department of Green Chemistry and Technology, Ghent University, Gent, Belgium, ${ }^{55}$ Department of Environment, Ghent University, Gent, Belgium, ${ }^{56}$ Department of Life and Environmental Sciences, Botany Division,
University of Cagliari, Cagliari, Italy, ${ }^{57}$ Helmholtz Centre for Environmental Research - UFZ, Leipzig, Germany, ${ }^{58}$ Royal Museum for Central Africa, Tervuren, Belgium, ${ }^{59}$ University of Bayreuth, Bayreuth, Germany, ${ }^{60}$ Groningen Institute of Archaeology (GIA), University of Groningen, Groningen, The Netherlands, ${ }^{61}$ Department of Biological Sciences, University of Tennessee, Knoxville, TN, USA, ${ }^{62}$ Rocky Mountain Biological Laboratory, Crested Butte, CO, USA, ${ }^{63}$ Département des Science, Université du Québec À Montréal, Montreal, QC, Canada, ${ }^{64}$ Department of Biogeography, University of Bayreuth, Bayreuth, Germany, ${ }^{65}$ Museo Nacional de Ciencias Naturales-CSIC, Madrid, Spain, ${ }^{66}$ Université Laval, Quebec, QC, Canada, ${ }^{67}$ Instituto de Ciencias Naturales, Universidad Nacional de Colombia, Bogota, Colombia, ${ }^{68}$ Fundación Natura, Bogota, Colombia, ${ }^{69}$ Environmental Change Institute, University of Oxford, Oxford, UK, ${ }^{70}$ Laboratório de Estudos em Vegetação Campestre (LEVCamp), Programa de Pós-Graduação em Botânica, Universidade Federal do Rio Grande do Sul, Porto Alegre, Brazil, ${ }^{71}$ Institut für Biologie, Freie Universität Berlin, Berlin, Germany, ${ }^{72}$ Berlin-Brandenburg Institute of Advanced Biodiversity Research (BBIB), Berlin, Germany, ${ }^{73}$ Laboratório de Ecologia Funcional de Comunidades (LABEF), Departamento de Botânica, Universidade Federal do Paraná, Curitiba, Brazil, ${ }^{74}$ School of Informatics, Computing, and Cyber Systems, Northern Arizona University, Flagstaff, AZ, USA, ${ }^{75}$ Institute of Ecology and Evolution, Friedrich Schiller University Jena, Jena, Germany, ${ }^{76}$ ETH Zurich, Zurich, Switzerland, ${ }^{77}$ Department of Biological and Environmental Sciences, University of Gothenburg, Gothenburg, Sweden, ${ }^{78}$ PIAF, INRA, Université Clermont-Auvergne, Clermont-Ferrand, France, ${ }^{79}$ Universidade Federal do Rio Grande do Sul, Porto Alegre, Brazil, ${ }^{80}$ School of Life Sciences, Arizona State University, Tempe, AZ, USA, ${ }^{81}$ USDA-ARS Rangeland Resources \& Systems Research Unit, Fort Collins, CO, USA, ${ }^{82}$ Grupo de Investigación en Biodiversidad y Dinámica de Ecosistémas Tropicales - Universidad del Tolima, Ibagué, Colombia, ${ }^{83}$ Isotope Bioscience Laboratory - ISOFYS, Ghent University, Gent, Belgium, ${ }^{84}$ School of Forest Resources and Conservation, University of Florida, Gainesville, FL, USA, ${ }^{85}$ Senckenberg Biodiversity and Climate Research Centre, Frankfurt am Main, Germany, ${ }^{86}$ Department of Biological Sciences, Goethe Universität Frankfurt, Frankfurt am Main, Germany, ${ }^{87}$ Department of Biological Sciences, University of Cape Town, Cape Town, South Africa, ${ }^{88}$ SAEON Fynbos Node, Claremont, South Africa, ${ }^{89}$ Pacific Northwest National Laboratory, College Park, MD, USA, ${ }^{90}$ School of Geography, Geology and Environment, University of Leicester, Leicester, UK, ${ }^{91}$ Department of Environmental Science, Institute for Water and Wetland Research, Radboud University, Nijmegen, The Netherlands, ${ }^{92}$ Laboratório de Ecologia Vegetal (LEVEG), Programa de Pós-Graduação em Ecologia, Universidade Federal do Rio Grande do Sul, Porto Alegre, Brazil, ${ }^{93}$ Archbold Biological Station's Buck Island Ranch, FL, Lake Placid, USA, ${ }^{94}$ Department of Ecology and Evolutionary Biology, University of Connecticut, Storrs, CT, USA, ${ }^{95}$ University of Tasmania, Hobart, Tas., Australia, ${ }^{96}$ Facultad de Ciencias Forestales, Universidad Nacional de Santiago del Estero, Santiago del Estero, Argentina, ${ }^{97}$ Institute of Landscape and Plant Ecology, University of Hohenheim, 
Stuttgart, Germany, ${ }^{98}$ School of Geography, University of Nottingham, Nottingham, UK, ${ }^{99}$ Department of Geography and Geology, Kingston University, Kingston upon Thames, UK, ${ }^{100}$ Institute of Biology/Geobotany and Botanical Garden, Martin Luther University Halle-Wittenberg, Halle, Germany, ${ }^{101}$ Facultad de Ingeniería y Ciencias Hídricas, Universidad Nacional del Litoral (FICH-UNL), Santa Fe, Argentina, ${ }^{102}$ AMAP, CIRAD, CNRS, IRD, INRA, Université de Montpellier, Montpellier, France, ${ }^{103}$ AMAP, IRD, Herbier de Nouvelle-Calédonie, Nouméa, New Caledonia, ${ }^{104}$ University of Toronto Scarborough, Scarborough, ON, Canada, ${ }^{105}$ Friedrich-Schiller-Universität Jena, Jena, Germany, ${ }^{106}$ Section for Ecoinformatics and Biodiversity, Department of Bioscience, Aarhus University, Aarhus, Denmark, ${ }^{107}$ Center for Biodiversity Dynamics in a Changing World (BIOCHANGE), Department of Bioscience, Aarhus University, Aarhus, Denmark, ${ }^{108} \mathrm{New}$ Jersey Institute of Technology, Newark, NJ, USA, ${ }^{109}$ Faculty of Agricultural and Environmental Sciences, University of Rostock, Rostock, Germany, ${ }^{110}$ Sapienza University of Rome, Rome, Italy, ${ }^{111}$ School of Biological Sciences, University of Aberdeen, Aberdeen, UK, ${ }^{112}$ Center for Ecosystem Science and Society, Northern Arizona University, Flagstaff, AZ, USA, ${ }^{113}$ School of Civil and Environmental Engineering, Yonsei University, Seoul, Korea, ${ }^{114}$ Departamento de Botânica, SCB, UFPR - Federal University of Parana, Curitiba, Brazil, ${ }^{115}$ Centro Politécnico, Universidade Federal do Paraná, Curitiba, Brazil, ${ }^{116}$ Dipartimento di Bioscienze, Università degli Studi di Milano, Milano, Italy, ${ }^{117}$ IRSTEA Aix-en-Provence, UMR RECOVER, Aix-Marseille University, Aix-enProvence, France, ${ }^{118}$ Department of Environmental Systems Science, ETH Zürich, Zürich, Switzerland, ${ }^{119}$ Swiss Federal Institute for Forest, Snow and Landscape Research WSL, Birmensdorf, Switzerland, ${ }^{120}$ Centre of Excellence for Bioscurity Risk Analysis, The University of Melbourne, Melbourne, Vic., Australia, ${ }^{121}$ Instituto Pirenaico de Ecología (IPE-CSIC), Zaragoza, Spain, ${ }^{122}$ Colgate University, Hamilton, NY, USA, ${ }^{123}$ School of Biosciences and Veterinary Medicine, Plant Diversity and Ecosystems Management Unit, University of Camerino, Camerino, Italy, ${ }^{124}$ Department of Plant Biology and Ecology, University of the Basque Country UPV/ EHU, Bilbao, Spain, ${ }^{125}$ Departamento de Geociencias y Medio Ambiente, Universidad Nacional de Colombia, Medellin, Colombia, ${ }^{126}$ Department of Chemistry, Life Sciences and Environmental Sustainability, University of Parma, Parma, Italy, ${ }^{127}$ CATIE-Centro Agronómico Tropical de Investigación y Enseñanza, Turrialba, Costa Rica, ${ }^{128}$ Univ. Bordeaux, INRAE, BIOGECO, Cestas, France, ${ }^{129}$ Department of Geography, King's College London, London, UK, ${ }^{130}$ Department of Ecology, Evolution, and Behavior, University of Minnesota, St. Paul, MN, USA, ${ }^{131}$ Department of Biotechnology and Life Sciences, University of Insubria, Varese, Italy, ${ }^{132}$ BIGEA, Department of Biological, Geological and Environmental Sciences, Alma Mater Studiorum - University of Bologna, Bologna, Italy, ${ }^{133}$ Escuela de Biología, Universidad de Costa Rica, San José, Costa Rica, ${ }^{134}$ The University of Arizona, Tucson, AZ, USA, ${ }^{135}$ Institute of Arctic Biology, University of Alaska Fairbanks, Fairbanks, AK, USA, ${ }^{136}$ Royal Botanic Gardens, Kew, West Sussex, UK, ${ }^{137}$ Department of Biology, Colorado State University, Fort Collins, CO, USA, ${ }^{138}$ WSL
Swiss Federal Research Institute, Birmensdorf, Switzerland, ${ }^{139}$ Faculty of Forestry, University of British Columbia, Vancouver, BC, Canada, ${ }^{140}$ CREA - Research Centre for Forestry and Wood, Arezzo, Italy, ${ }^{141}$ Hawkesbury Institute for the Environment, Western Sydney University, Sydney, NSW, Australia, ${ }^{142}$ Jungwon University, Goesan, Chungbuk, Korea, ${ }^{143}$ Department of Botany and Zoology, Masaryk University, Brno, Czech Republic, ${ }^{144}$ Department of Biology, University of Pisa, Pisa, Italy, ${ }^{145}$ Department of Agriculture and Forest Engineering (EAGROF), University of Lleida, Lleida, Spain, ${ }^{146}$ Joint Research Unit CTFC - AGROTECNIO, Solsona, Spain, ${ }^{147}$ University of California Riverside, Riverside, CA, USA, ${ }^{148}$ Faculty of Environmental Sciences, University of Life Sciences Prague, Praha-Suchdol, Czech Republic, ${ }^{149}$ Institute of Botany, Czech Academy of Sciences, Třeboň, Czech Republic, ${ }^{150}$ Department of Plant Sciences, University of Cambridge, Cambridge, UK, ${ }^{151}$ Systems Ecology, Department of Ecological Science, Vrije Universiteit, Amsterdam, The Netherlands, ${ }^{152}$ School of Biological, Earth and Environmental Sciences, UNSW Sydney, Sydney, NSW, Australia, ${ }^{153}$ Faculté de foresterie, de géographie et de géomatique, Université Laval, Quebec, QC, Canada, ${ }^{154}$ Jonah Ventures, Boulder, CO, USA, ${ }^{155}$ Centro de Modelación y Monitoreo de Ecosistemas, Universidad Mayor, Santiago, Chile, ${ }^{156}$ Department of Wildlife, Fish and Environmental Studies, Swedish University of Agricultural Sciences, Umeå, Sweden, ${ }^{157}$ Centre for African Conservation Ecology, Department of Zoology, Nelson Mandela University, Port Elizabeth, South Africa, ${ }^{158}$ MTA Centre for Ecological Research, Tihany, Hungary, ${ }^{159}$ Biotechnical Faculty, University of Ljubljana, Ljubljana, Slovenia, ${ }^{160}$ Université de Lorraine, AgroParisTech, INRAE, UMR Silva, Nancy, France, ${ }^{161}$ Santa Catarina State University, Lages, SC, Brazil, ${ }^{162}$ Department of Geography, Environment, and Spatial Sciences, Michigan State University, East Lansing, MI, USA, ${ }^{163}$ Eurac Research, Institute for Alpine Environment, Bozen-Bolzano, Italy, ${ }^{164}$ Institute of Biology of Komi Science Centre of the Ural Branch of the Russian Academy of Sciences, Syktyvkar, Komi Republic, Russia, ${ }^{165}$ University of Science - Vietnam National University Ho Chi Minh City, Ho Chi Minh City, Vietnam, ${ }^{166}$ Graduate School of Agriculture, Kyoto University, Kyoto, Japan, ${ }^{167}$ Graduate School of Global Environmental Studies, Kyoto University, Kyoto, Japan, ${ }^{168}$ Swedish Species Information Centre, Swedish University of Agricultural Sciences, Uppsala, Sweden, ${ }^{169}$ Department of Plant and Soil Sciences, University of Pretoria, Pretoria, South Africa, ${ }^{170}$ Department of Terrestrial Ecology, Netherlands Institute of Ecology, Wageningen, The Netherlands, ${ }^{171}$ Department Computational Landscape Ecology, UFZ - Helmholtz Centre for Environmental Research, Leipzig, Germany, ${ }^{172}$ Department Computational Hydrosystems, UFZ - Helmholtz Centre for Environmental Research, Leipzig, Germany, ${ }^{173}$ Department of Landscape Architecture and Rural Systems Engineering, Seoul National University, Seoul, Republic of Korea, ${ }^{174}$ Institute of Temperate Forest Sciences (ISFORT), Ripon, QC, Canada, ${ }^{175}$ UQO, Department of Natural Sciences, Ripon, QC, Canada, ${ }^{176}$ Cirad, UMR EcoFoG (Agroparistech, CNRS, INRA, Université des Antilles, Université de la Guyane), Kourou, French Guiana, France, ${ }^{177}$ Institut 
für Physische Geographie, Biogeography and Biodiversity Lab, Goethe-Universität Frankfurt, Frankfurt am Main, Germany, ${ }^{178}$ Department of Biology, University of Florida, Gainesville, FL, USA, ${ }^{179}$ lluka Resources, Perth, WA, Australia, ${ }^{180}$ School of Biological Sciences, The University of Western Australia, Perth, WA, Australia, ${ }^{181}$ Department of Biological Sciences, Macquarie University, Sydney, NSW, Australia, ${ }^{182}$ Department of Botany and Molecular Evolution, Senckenberg Research Institute and Natural History Museum, Frankfurt am Main, Germany, ${ }^{183}$ Department of Botany and Biodiversity Research, University of Vienna, Vienna, Austria, ${ }^{184}$ Helmholtz Centre for Environmental Research - UFZ, Halle, Germany, ${ }^{185}$ Department of Botany, Faculty of Sciences, University of South Bohemia, Ceske Budejovice, Czech Republic, ${ }^{186}$ Department of Environmental and Life Sciences - Biology, Karlstad University, Karlstad, Sweden, ${ }^{187}$ Quantitative Plant Ecology and Biodiversity Research Laboratory, Department of Biology, Faculty of Science, Ferdowsi University of Mashhad, Mashhad, Iran, ${ }^{188}$ Flathead Lake Biological Station, University of Montana, Polson, MT, USA, ${ }^{189}$ School of Sustainability, Arizona State University, Tempe, AZ, USA, ${ }^{190}$ Programa Nacional de Pós-Doutorado (PNPD), Programa de Pós Graduação em Ecologia, Institute of Biology, University of Campinas UNICAMP, Brazil, ${ }^{191}$ Institute for Ecosystem Research/ Geobotany, Kiel University, Kiel, Germany, ${ }^{192}$ School of Geography, Earth and Environmental Sciences - University of Birmingham, Birmingham, UK, ${ }^{193}$ Institute for Plant Ecology, Justus Liebig University, Giessen, Germany, ${ }^{194}$ Spanish National Research Council - CSIC, Catalonia, Spain, ${ }^{195}$ CREAF, Catalonia, Spain, ${ }^{196}$ Department of Biology - FFCLRP/USP, Ribeirão Preto, Brazil, ${ }^{197}$ University of Maryland, College Park, MD, USA, ${ }^{198}$ Campus da Zapateira, University of A Coruña, A Coruña, Spain, ${ }^{199}$ Evolution \& Ecology Research Centre, and School of Biological, Earth and Environmental Sciences, UNSW Sydney, Sydney, NSW, Australia, ${ }^{200}$ Rutgers University, Piscataway, NJ, USA, ${ }^{201}$ Peking University, Beijing, China, ${ }^{202}$ Department of Chemistry and Pharmacy, University of Sassari, Sassari, Italy, ${ }^{203}$ Faculty of Arts and Sciences, Molecular Biology and Genetics, Ordu University, Ordu, Turkey, ${ }^{204}$ State Key Laboratory of Grassland Agro-ecosystems, College of Pastoral Agriculture Science and Technology, Lanzhou University, Lanzhou, China, ${ }^{205}$ Centro Forestal Tropical Bajo Calima, Universidad del Tolima, Buenaventura, Colombia, ${ }^{206}$ Embrapa Amazônia Oriental, Belém, Brazil, ${ }^{207}$ Instituto de Biociências, Laboratory of Vegetation Ecology, Universidade Estadual Paulista (UNESP), Rio Claro, Brazil, ${ }^{208}$ Queensland University of Technology (QUT), Brisbane, Australia, ${ }^{209}$ School of Life Sciences, University of Sussex, Brighton, UK, ${ }^{210}$ Arnold Arboretum of Harvard University, Boston, MA, USA, ${ }^{211}$ Laboratoire ECODIV URA, IRSTEA/EA 1293, Normandie Université, UFR ST, Université de Rouen, Mont Saint-Aignan, France, ${ }^{212}$ Department of Botany, Biosciences Institute, Federal University of Rio Grande do Sul, Porto Alegre, Brazil, ${ }^{213}$ Unit of Research SPHERES, University of Liège, Liège, Belgium, ${ }^{214}$ Géopole de I'Université de Sherbrooke, Quebec, QC, Canada, ${ }^{215}$ Theoretical and Experimental Ecology Station, CNRS, Paul Sabatier University Toulouse, Moulis, France, ${ }^{216}$ School of Earth and Environment Science, University of
Manchester, Manchester, UK, ${ }^{217}$ Department of Plant Biology, Institute of Biology, University of Campinas, Campinas, Brazil, ${ }^{218}$ Universidade Federal do Rio Grande do Norte - UFRN, Natal, RN, Brazil, ${ }^{219}$ Departamento de Biología y Geología, Física y Química Inorgánica y Analítica, Universidad Rey Juan Carlos, Móstoles, Spain, ${ }^{220}$ Instituto Nacional de Tecnología Agropecuaria, Consejo Nacional de Invetigaciones Científicas y Técnicas, Universidad Nacional de La Patagonia Austral, Río Gallegos, Argentina, ${ }^{221}$ UMR 5175 CEFE, Univ. Montpellier, CNRS, EPHE, IRD, Univ. Paul Valéry, Montpellier, France, ${ }^{222}$ Estación Experimental del Zaidín, Consejo Superior de Investigaciones Científicas, Granada, Spain, ${ }^{223}$ Estación Biológica de Doñana, Consejo Superior de Investigaciones Científicas, Sevilla, Spain, ${ }^{224}$ Universidade Regional de Blumenau, Blumenau, SC, Brazil, ${ }^{225}$ INIA-CIFOR, Madrid, Spain, ${ }^{226}$ School of Biological Sciences, Southern Illinois University Carbondale, Carbondale, IL, USA, ${ }^{227}$ Center for Biodiversity Management, Yungaburra, QId, Australia, ${ }^{228}$ Instituto Federal de Educação Ciência e Tecnologia do Ceará, Crateús, Brazil, ${ }^{229}$ The Morton Arboretum, Lisle, IL, USA, ${ }^{230}$ Water Management and Systems Research Unit, United States Department of Agriculture, Agricultural Research Service, Fort Collins, CO, USA, ${ }^{231}$ Institute of Integrative Biology, ETH Zürich (Swiss Federal Institute of Technology), Zürich, Switzerland, ${ }^{232}$ Department of Ecology, Evolution \& Behavior, University of Minnesota, Minneapolis, MN, USA, ${ }^{233}$ Smithsonian Conservation Biology Institute, Front Royal, VA, USA, ${ }^{234}$ CSIC - Institute for Sustainable Agriculture (IAS), Cordoba, Spain, ${ }^{235}$ Facultad de Ciencias Naturales y Matemáticas, Universidad del Rosario, Bogota, Colombia, ${ }^{236}$ Departamento de Biología Animal, Biología Vegetal y Ecología, Universidad de Jaén, Jaén, Spain, ${ }^{237}$ Norwegian University of Science and Technology NTNU, Trondheim, Norway, ${ }^{238}$ Department of Life Sciences, University of Alcalá, Alcala de Henares, Spain, ${ }^{239}$ University of Florida, Gainesville, FL, USA, ${ }^{240}$ Department of Organismic and Evolutionary Biology, Harvard University, Cambridge, MA, USA, ${ }^{241}$ UCA, INRA, VetAgro Sup, UMR Ecosystème Prairial, ClermontFerrand, France, ${ }^{242}$ Departamento de Biología y Geología, Física y Química Inorgánica, Escuela Superior de Ciencias Experimentales y Tecnología, Universidad Rey Juan Carlos, Móstoles, Spain, ${ }^{243}$ Departamento de Ciencias Ambientales y Recursos Naturales Renovables, Facultad de Ciencias Agronómicas, Universidad de Chile, Santiago, Chile, ${ }^{244}$ Pacific Northwest National Laboratory, Joint Global Change Research Institute, College Park, MD, USA, ${ }^{245}$ The University of Manchester, Manchester, UK, ${ }^{246}$ Smithsonian Tropical Research Institute, Balboa, Ancon, Republic of Panama, ${ }^{247}$ Unit of research SPHERES, University of Liège, Liège, Belgium, ${ }^{248}$ College of Resources and Environmental Sciences, China Agricultural University, Beijing, China, ${ }^{249}$ Xinjiang Institute of Ecology and Geography, Chinese Academy of Sciences, Urumqi, China, ${ }^{250}$ Research Center for Ecology and Environment of Central Asia, Chinese Academy of Sciences, Urumqi, China, ${ }^{251}$ University of Reading, Reading, UK, ${ }^{252}$ School of Animal, Plant and Environmental Sciences, University of the Witwatersrand, Johannesburg, South Africa, ${ }^{253}$ Applied Ecology Research Group, School of Life Sciences, Anglia Ruskin University, Cambridge, UK, ${ }^{254}$ Faculty of Environmental 
Sciences and Natural Resource Management, Norwegian University of Life Sciences, Ås, Norway, ${ }^{255}$ School of Molecular and Life Sciences, Curtin University, Perth, WA, Australia, ${ }^{256}$ College of Science, Health, Engineering and Education, Murdoch University, Murdoch, WA, Australia, ${ }^{257}$ South China Botanical Garden, Chinese Academy of Sciences, Guangzhou, China, ${ }^{258}$ Carnegie Museum of Natural History, Pittsburgh, PA, USA, ${ }^{259}$ Institute of Ecology and Earth Sciences, University of Tartu, Tartu, Estonia, ${ }^{260}$ Herbarium Haussknecht, Friedrich-Schiller-Universität Jena, Jena, Germany, ${ }^{261}$ Cirad, Université de Montpellier, Montpellier, France, ${ }^{262}$ Institut National Polytehcnique Félix Houphouet-Boigny, INP-HB, Yamoussoukro, Ivory Coast, ${ }^{263}$ Department of Forest Sciences, Transilvania University of Brasov, Brasov, Romania, ${ }^{264} \mathrm{BC} 3$ - Basque Centre for Climate Change, Scientific Campus of the University of the Basque Country, Leioa, Spain, ${ }^{265}$ Department of Physical Geography, Goethe University, Frankfurt am Main, Germany, ${ }^{266}$ Institute of Botany, University of Natural Resources and Life Sciences, Vienna, Austria, ${ }^{267}$ The Field Museum, Chicago, IL, USA ${ }^{268}$ University Centre Myerscough, Preston, UK, ${ }^{269}$ Department of Biological Sciences, Florida International University, Miami, FL, USA ${ }^{270}$ Oak Ridge National Laboratory, US Department of Energy, Oak Ridge, TN, ${ }^{271}$ University of California, Santa Cruz, Santa Cruz, CA, USA, ${ }^{272}$ Plant Conservation and Population Biology, Department of Biology, KU Leuven, Leuven, Belgium, ${ }^{273}$ Division of Ecology, Evolution and Biodiversity Conservation, Heverlee, Belgium, ${ }^{274}$ Four Peaks Environmental Science and Data Solutions, Wenatchee, WA, USA, ${ }^{275}$ Department of Landscape and Biodiversity, Norwegian Institute of Bioeconomy Research (NIBIO), Ås, Norway, ${ }^{276}$ Kochi University, Nankoku, Japan, ${ }^{277}$ University of Illinois at Chicago, Chicago, IL, USA, ${ }^{278}$ Department of Evolutionary Biology, Ecology and Environmental Sciences, Biodiversity Research Institute (IRBio), Universitat de Barcelona, Barcelona, Spain, ${ }^{279}$ University of Toronto, Toronto, ON, Canada, ${ }^{280}$ Ashiu Forest Research Station, Field Science Education and Research Center, Kyoto University, Kyoto, Japan, ${ }^{281}$ Institute Botanic Garden, Ural Branch of the Russian Academy of Sciences, Ekaterinburg, Russia, ${ }^{282}$ Tyumen State University, Tyumen, Russia, ${ }^{283}$ Oak Ridge National Laboratory, Oak Ridge, TN, USA, ${ }^{284}$ Barcelona School of Agricultural Engineering, Universitat Politècnica de Catalunya, Catalonia, Spain, ${ }^{285}$ Earth System Science Department, Stanford University, Stanford, CA USA, ${ }^{286} \mathrm{Global}$ Academy of Agriculture and Food Security, University of Edinburgh, Midlothian, Scotland, ${ }^{287}$ Institute of Dendrology, Polish Academy of Sciences, Kornik, Poland, ${ }^{288}$ Department of Game Management and Forest Protection, Faculty of Forestry, Poznan University of Life Sciences, Poznan, Poland, ${ }^{289}$ Geobotany and Botanical Garden, Martin Luther University Halle-Wittenberg, Halle, Germany, ${ }^{290}$ Institute of Systematic Botany and Ecology, Ulm University, Ulm, Germany, ${ }^{291}$ BayCEER, Department of Disturbance Ecology, University of Bayreuth, Bayreuth, Germany, ${ }^{292}$ Department of Biology, University of Copenhagen, Frederiksberg C, Denmark, ${ }^{293}$ Plant Ecophysiology \& Evolution Group, Guangxi Key Laboratory of Forest Ecology and Conservation, College of Forestry, Guangxi University, Nanning, Guangxi, PR China, ${ }^{294}$ State Key Laboratory for
Conservation and Utilization of Subtropical Agro-bioresources, Guangxi University, Nanning, PR China, ${ }^{295}$ Terrestrial Ecology Section, Department of Biology, University of Copenhagen, Denmark, ${ }^{296}$ State University of Campinas/UNICAMP, Campinas, SP, Brazil, ${ }^{297}$ Biological Sciences, University of Tasmania, Hobart, Australia, ${ }^{298}$ Department of Zoology, School of Mathematical and Natural Science, University of Venda, Thohoyandou, South Africa, ${ }^{299}$ Department of Biological Sciences, DST/NRF Centre of Excellence, Percy FitzPatrick Institute of African Ornithology, University of Cape Town, Rondebosch, South Africa, ${ }^{300}$ Cibodas Botanical Garden - Indonesian Institute of Sciences (LIPI), JI. Kebun Raya Cibodas, Cipanas, Indonesia, ${ }^{301}$ Centre of Excellence for Biosecurity Risk Analysis (CEBRA), School Of Biosciences, University of Melbourne, Parkville, Vic., Australia, ${ }^{302}$ Evolutionary Ecology of Plants, Department Biology, Philipps-University Marburg, Marburg, Germany, ${ }^{303}$ Department of Bioscience, University Salzburg, Salzburg, Austria, ${ }^{304}$ PERSYST Department, CIRAD, Montpellier Cedex 5, France, ${ }^{305} \mathrm{BC}$ Ministry Forest, Lands, Natural Resource Operations and Rural Development, Dawson Creek, BC, Canada, ${ }^{306}$ Humboldt State University, Arcata, CA, USA, ${ }^{307}$ Institute of Botany, The Czech Academy of Sciences, Prùhonice, Czech Republic, ${ }^{308}$ Department of Botany, Faculty of Science, Charles University, Prague, Czech Republic, ${ }^{309}$ Institute of Geography and Geoecology, Karlsruhe Institute of Technology, Karlsruhe, Germany, ${ }^{310}$ Samara National Research University, Samara, Russia, ${ }^{311}$ CAVElab Computational and Applied Vegetation Ecology, Ghent University, Ghent, Belgium, ${ }^{312}$ Institute of Plant Sciences, Bern, Switzerland, ${ }^{313}$ Forestry and Forest Products Research Institute, Tsukuba, Japan, ${ }^{314}$ Kenyon College, Gambier, OH, USA, ${ }^{315}$ Department of Biology, University of Garmian, Kalar, Iraq, ${ }^{316}$ School of Biological Sciences and Center for Ecology, Southern Illinois University Carbondale, Carbondale, IL, USA, ${ }^{317}$ Department of Ecology and Evolution, State University of New York at Stony Brook, Stony Brook, NY, USA, ${ }^{318}$ Institute for Biodiversity and Ecosystem Dynamics (IBED), University of Amsterdam, Amsterdam, The Netherlands, ${ }^{319}$ Instituto de Investigaciones en Biodiversidad y Medioambiente (INIBIOMA), CONICET, Bariloche, Argentina, ${ }^{320}$ Departamento de Ecología, Universidad Nacional del Comahue, Bariloche, Argentina, ${ }^{321}$ Department of Plant and Environmental Sciences, Weizmann Institute of Science, Rehovot, Israel, ${ }^{322}$ Landscape Ecology Group, Institute of Biology and Environmental Sciences, University of Oldenburg, Oldenburg, Germany, ${ }^{323}$ Faculty of Sciences, Charles University, Praha, Czech Republic, ${ }^{324}$ Laboratório de Ecologia Vegetal (LEVEG), Programa de Pós-Graduação em Ecologia, Universidade Federal do Rio Grande do Sul, Porto Alegre, Brazil, ${ }^{325}$ Department of Geosciences and Natural Resources, Western Carolina University, Cullowhee, NC, USA, ${ }^{326}$ Department of Community Ecology, Helmholtz Centre for Environmental ResearchUFZ, Halle (Saale), Germany, ${ }^{327}$ Health and Environmental Sciences, Xi'an Jiaotong Liverpool University, Suzhou, Jiangsu, China, ${ }^{328}$ Hokkaido University, Sapporo, Japan, ${ }^{329}$ Graduate School of Environment and Information Sciences, Yokohama National University, Yokohama, Japan, ${ }^{330}$ Technical University of Munich, 
Freising, Germany, ${ }^{331}$ Institut d Estudis Andorrans, Andorra, ${ }^{332}$ Smithsonian Environmental Research Center, Edgewater, MD, USA, ${ }^{333}$ Department of Geography, Humboldt University of Berlin, Berlin, Germany, ${ }^{334}$ Biodiversity, Macroecology and Biogeography, University of Goettingen, Göttingen, Germany, ${ }^{335}$ Department of Ecology and Evolutionary Biology, University of California, Los Angeles, CA, USA, ${ }^{336}$ Wageningen University \& Research, Wageningen, The Netherlands, ${ }^{337}$ Land Life Company, Amsterdam, The Netherlands, ${ }^{338}$ Centre of Biodiversity and Sustainable Land Use (CBL), University of Goettingen, Göttingen, Germany, ${ }^{339}$ Martin Luther University Halle-Wittenberg, Halle, Germany, ${ }^{340}$ Plant Physiology Division, Coconut Research Institute of Sri Lanka, Lunuwila, Sri Lanka, ${ }^{341}$ Institute of Evolutionary Ecology and Conservation Genomics, Ulm University, Ulm, Germany, ${ }^{342}$ Yamagata University, Yamagata, Japan, ${ }^{343}$ Jomo Kenyatta University of Agriculture and Technology (JKUAT), Nairobi, Kenya, ${ }^{344} \mathrm{CIRAD}$, UMR Eco\&Sols, Montpellier, France, ${ }^{345}$ Eco\&Sols, CIRAD, INRA, IRD, SupAgro, University of Montpellier, Montpellier, France, ${ }^{346}$ Institut de recherche sur les forêts, Université du Québec en Abitibi-Témiscamingue, Rouyn-Noranda, QC, Canada, ${ }^{347}$ Department of Plant Sciences, University of Saskatchewan, Saskatoon, SK, Canada, ${ }^{348}$ GLORIA-Coordination, Institute for Interdisciplinary Mountain Research, Austrian Academy of Sciences \& Department of Integrative Biology and Biodiversity Research, University of Natural Resources and Life Sciences Vienna, Vienna, Austria, ${ }^{349}$ Department of Fisheries, Wildlife and Conservation Biology, University of Minnesota, St. Paul, MN, USA, ${ }^{350}$ Botany Department, University of Wyoming, Laramie, WY, USA, ${ }^{351}$ Aix Marseille Univ, Univ Avignon, CNRS, IRD, IMBE, Marseille, France, ${ }^{352}$ Nelson Mandela University, Port Elizabeth, South Africa, ${ }^{353}$ University of Wisconsin Eau Claire, Eau Claire, WI, USA, ${ }^{354}$ Naturalis Biodiversity Center, Leiden, The Netherlands, ${ }^{355}$ Department of Geography, University College London, London, UK, ${ }^{356}$ Sun Yat-sen University, Guangzhou, China, ${ }^{357}$ Yangzhou University, Yangzhou, Jiangsu, China, ${ }^{358}$ University of Leipzig, Leipzig, Germany, ${ }^{359}$ Macquarie University, North Ryde, NSW, Australia, ${ }^{360}$ University Pablo de Olavide, Seville, Spain, ${ }^{361}$ School of Agriculture and Biology, Shanghai Jiao Tong University, Shanghai, P. R. China, ${ }^{362}$ Shanghai Urban Forest Ecosystem Research Station, National Forestry and Grassland Administration, Shanghai, P.R. China, ${ }^{363}$ Universitat Autònoma de Barcelona, Barcelona, Spain, ${ }^{364}$ Forest Ecology and Forest Management Group, Wageningen University and Research, Wageningen, The Netherlands, ${ }^{365}$ World Agroforestry (ICRAF), Nairobi, Kenya, ${ }^{366}$ Dept. Animal Biology, Plant Biology and Ecology, University of Jaén, Jaén, Spain, ${ }^{367}$ water@ leeds, School of Geography, University of Leeds, Leeds, UK, ${ }^{368}$ Department for Tisza River Research, MTA Centre for Ecological Research, DRI, Debrecen, Hungary, ${ }^{369}$ Global Change Research Institute AS CR, Brno, Czech Republic, ${ }^{370}$ Department of Ecology, School of Horticulture and Plant Protection, Yangzhou University, Yangzhou, China, ${ }^{371}$ State Key Laboratory of Urban and Regional Ecology, Research Centre for Eco-Environmental Sciences, Chinese Academy of Sciences, Beijing, China, ${ }^{372}$ University of Copenhagen,
Copenhagen, Denmark, ${ }^{373}$ Université du Québec à Trois-Rivières, Trois-Rivières, Canada, ${ }^{374}$ Institute of Atmospheric and Earth System Research (INAR), University of Helsinki, Helsinki, Finland, ${ }^{375}$ Natural Resources Institute Finland, Espoo, Finland, ${ }^{376}$ Federal University of Alagoas, Maceió, AL, Brazil, ${ }^{377}$ Lakehead University, Thunder Bay, ON, Canada, ${ }^{378}$ Department of Physical Geography, Stockholm University, Stockholm, Sweden, ${ }^{379}$ Bolin Centre for Climate Research, Stockholm, Sweden, ${ }^{380}$ Universidade Federal do Paraná, Curitiba, PR, Brazil, ${ }^{381}$ School of Natural Sciences, Bangor University, Bangor, UK, ${ }^{382}$ Department of Physical and Environmental Sciences, University of Toronto Scarborough, Toronto, ON, Canada, ${ }^{383}$ Centro de Investigación en Biodiversidad y Conservación, Universidad Autónoma del Estado de Morelos, Morelos, Mexico, ${ }^{384}$ Universitat Autònoma de Barcelona, Catalonia, Spain, ${ }^{385}$ Centre for Ecology \& Hydrology, Lancaster Environment Centre, Lancaster, UK, ${ }^{386}$ Manaaki Whenua - Landcare Research, Hamilton, New Zealand, ${ }^{387}$ Department of Scientific Services, Gorongosa National Park, Beira, Sofala Province, Mozambique, ${ }^{388}$ Institut de recherche en biologie végétale, Montréal, QC, Canada, ${ }^{389}$ Université de Montréal, Montréal, QC, Canada, ${ }^{390}$ School of Plant Sciences and Food Security, George S. Wise Faculty of Life Sciences, Tel Aviv University, Tel Aviv, Israel, ${ }^{391}$ School of Biological Sciences, The University of Queensland, Brisbane, QId, Australia, ${ }^{392}$ CSIRO, Canberra, ACT, Australia, ${ }^{393}$ Manaaki Whenua - Landcare Research, Lincoln, New Zealand, ${ }^{394}$ Center For Tree Science, The Morton Arboretum, Lisle, IL, USA, ${ }^{395}$ Department of Botany, University of Wisconsin-Madison, Madison, WI, USA, ${ }^{396}$ University of Maine, Orono, ME, USA, ${ }^{397}$ Department of Geography, Environment \& Society, University of Minnesota, Minneapolis, MN, USA, ${ }^{398} \mathrm{Holden}$ Arboretum, Kirtland, Ohio, USA, ${ }^{399}$ Université Libre de Bruxelles, Bruxelles, Belgium, ${ }^{400}$ Institute for Resources Environment and Sustainability, University of British Columbia, Vancouver, BC, Canada, ${ }^{401}$ Research School of Biology, The Australian National University, Canberra, ACT, Australia, ${ }^{402}$ School of Geosciences, The University of Edinburgh, Edinburgh, UK, ${ }^{403}$ Universidade Federal de Pernambuco, Recife, PE, Brazil, ${ }^{404}$ ICREA, Barcelona, Spain, ${ }^{405}$ CREAF, Barcelona, Spain, ${ }^{406}$ INRA, UEFP, Cestas, France, ${ }^{407}$ Department of Biology, University of Waterloo, Waterloo, ON, Canada, ${ }^{408}$ Department of Botany, Faculty of Science and Technology, University of Debrecen, Debrecen, Hungary, ${ }^{409}$ Natural Resources Canada, Canadian Forest Service, Northern Forestry Centre, Edmonton, AB, Canada, ${ }^{410}$ Department of Botany and Biodiversity Research Centre, University of British Columbia, Vancouver, BC, Canada, ${ }^{411}$ Tulipan $\mathrm{s} / \mathrm{n}$, Móstoles-Madrid, Spain, ${ }^{412}$ Department of Biology, Stanford University, Stanford, CA, USA, ${ }^{413}$ Institute of Biology and Environmental Sciences, University of Oldenburg, Oldenburg, Germany, ${ }^{414}$ Department of Biology, Vrije Universiteit Brussel, Brussels, Belgium, ${ }^{415}$ University of Illinois at Urbana-Champaign, Urbana-Champaign, IL, USA, ${ }^{416}$ CSIRO, Canberra, ACT, Australia, ${ }^{417}$ Department of Botany, University of Debrecen, Hungary, ${ }^{418}$ University of Vermont, Burlington, VT, USA, ${ }^{419}$ Fundação Zoobotânica do Rio Grande do Sul, Porto Allegre, Brazil, ${ }^{420}$ University of Minnesota, St. Paul, MN, USA, ${ }^{421}$ Gembloux Agro-Bio Tech, 
Biodiversity and landscape, University of Liège, Belgium, ${ }^{422}$ Department of Invasion Ecology, Institute of Botany, Czech Academy of Sciences, Průhonice, Czech Republic, ${ }^{423}$ Numerical Terradynamic Simulation Group (NTSG), College of Forestry and Conservation, University of Montana, Missoula, USA, ${ }^{424}$ Ontario Ministry of Natural Resources and Forestry, Centre for Northern Forest Ecosystem Research, Thunder Bay, ON, Canada, ${ }^{425}$ Universitat Politècnica de Catalunya, Castelldefels, Spain, ${ }^{426}$ Harry Butler Institute, Murdoch University, Perth, WA, Australia, ${ }^{427}$ Dept of Geography \& Environmental Studies, Stellenbosch University, Matieland, Stellenbosch, South Africa, ${ }^{428}$ Geobotany, Faculty of Biology, University of Freiburg, Freiburg im Breisgau, Germany, ${ }^{429}$ Department of Botany, University of Hawai'i, Honolulu, HI, USA ${ }^{430}$ Laboratório de Ecologia Vegetal, Departamento de Ecologia, Programa de Pós-Graduação em Ecologia, Universidade Federal do Rio Grande do Sul, Porto Alegre, RS, Brazil, ${ }^{431}$ Laboratoire d'Ecologie Alpine, Université Grenoble-Alpes, Grenoble Cedex 9, France, ${ }^{432}$ French Institute of Pondicherry, Puducherry, India, ${ }^{433}$ Biology Department, Oklahoma State University, Oklahoma, OK, USA ${ }^{434}$ Osaka City University, Osaka, Japan, ${ }^{435}$ Department of Ecology, French Institute of Pondicherry, Pondicherry, India, ${ }^{436}$ Galápagos Verde 2050, Charles Darwin Foundation, Charles Darwin Research Station, Galapagos, Ecuador, ${ }^{437}$ Forest, Rangeland, and Fire Sciences Department, University of Idaho, Moscow, ID, USA, ${ }^{438}$ College of Chemistry and Life Sciences, Zhejiang Normal University, Jinhua, China, ${ }^{439}$ Instituto de Investigación de Recursos Biológicos Alexander von Humboldt, Bogota, Colombia, ${ }^{440}$ Universidad Distrital Francisco José de Caldas, Bogota, Colombia, ${ }^{441}$ Estonian University of Life Sciences, Tartu, Estonia, ${ }^{442}$ Plant Ecology, University of Bayreuth, Bayreuth, Germany, ${ }^{443}$ Faculty of Biosciences and Aquaculture, NORD University, Steinkjer, Norway, ${ }^{444}$ Department of Biology, Norwegian University of Science and Technology, NTNU, Trondheim, Norway, ${ }^{445}$ CSIRO Land and Water, Hobart Tas, Tas., Australia, ${ }^{446}$ Institute of Applied Ecology, University of Canberra, Canberra, ACT, Australia, ${ }^{447}$ University of Innsbruck, Innsbruck, Austria, ${ }^{448}$ River Basin Research Center, Gifu University, Gifu, Gifu, Japan, ${ }^{449}$ Departamento de Botânica, Universidade Federal do Paraná, Curitiba, Brazil, ${ }^{450}$ Institute of Biology Bucharest, Romanian Academy, Bucharest, Romania, ${ }^{451}$ Institute of Ecology and Botany, MTA Centre for Ecological Research, Vácrátót, Hungary, ${ }^{452}$ Instituto de Biología, Tercer Circuito s/n de Ciudad Universitaria, Mexico City, Mexico, ${ }^{453}$ Universidad Nacional Autónoma de México, Coyoacán, Mexico, ${ }^{454}$ Department of Ecology and Plant Geography, Faculty of Biology, Moscow Lomonosov State University, Moscow, Russia, ${ }^{455}$ Kyoto University, Kyoto, Japan, ${ }^{456}$ Facultad de Ingeniería Agroindustrial, Universidad de las Américas, Quito, Equador, ${ }^{457}$ Meijo University, Nagoya, Aichi, Japan, ${ }^{458}$ Department of Zoology, University of British Columbia, Vancouver, BC, Canada, ${ }^{459}$ Wageningen Environmental Research, Wageningen, The Netherlands, ${ }^{460}$ Restoration Ecology, Technische Universität München, München, Germany, ${ }^{461}$ University of New England, Armidale, NSW, Australia, ${ }^{462}$ The James Hutton Institute, Aberdeen, UK, ${ }^{463}$ Department for Molecular Biology and Genetics, Democritus
University of Thrace, Alexandroupolis, Greece, ${ }^{464}$ University of Tartu, Tartu, Estonia, ${ }^{465}$ Instituto de Ciencias Ambientales y Evolutivas, Universidad Austral de Chile, Valdivia, Chile, ${ }^{466}$ Desertification Research Center (CIDE-CSIC), Valencia, Spain, ${ }^{467}$ Departamento de Ecología, Centro de Investigación en Biodiversidad y Cambio Global (CIBC), Universidad Autónoma de Madrid, Madrid, Spain, ${ }^{468}$ Global Ecology Unit CREAF-CSIC, Universitat Autònoma de Barcelona, Barcelona, Spain, ${ }^{469}$ University of Jaén, Jaén, Spain, ${ }^{470}$ Instituto Nacional de Tecnología Agropecuaria (INTA), Río Gallegos, Santa Cruz, Argentina, ${ }^{471}$ Universidad Nacional de la Patagonia Austral (UNPA), CONICET, Rio Gallegos, Argentina, ${ }^{472}$ Pós-graduação em Ecologia e Conservação, Universidade Federal do Paraná, Curitiba, PR, Brazil, ${ }^{473}$ National Institute for ResearchDevelopment in Forestry, Voluntari, Romania, ${ }^{474}$ Department of Agricultural and Environmental Sciences (DiSAA), University of Milan, Milano, Italy, ${ }^{475}$ Department of Ecology, Universidade Federal do Rio Grande do Sul, Porto Alegre, RS, Brazil, ${ }^{476}$ Botanical Garden of the Samara University, Samara, Russia, ${ }^{477}$ Plant Sciences (IBG2), Forschungszentrum Jülich $\mathrm{GmbH}$, Jülich, Germany, ${ }^{478}$ Institute of Ecology, Tallinn University, Tallinn, Estonia, ${ }^{479}$ Ecology and Conservation Biology, Institute of Plant Sciences, University of Regensburg, Regensburg, Germany, ${ }^{480}$ McGill University, Montreal, QC, Canada, ${ }^{481}$ Morton Arboretum, Lisle, IL, USA, ${ }^{482}$ Environmental Sciences Division \& Climate Change Science Institute, Oak Ridge National Laboratory, Oak Ridge, TN, USA, ${ }^{483}$ Research Unit ECOBIO - Ecosystèmes, Biodiversité, Evolution, Université Rennes 1/CNRS, Rennes, France, ${ }^{484}$ Department of Ecology, Faculty of Science, Charles University, Prague, Czech Republic, ${ }^{485}$ CEFE, CNRS, EPHE, Université de Montpellier, Université Paul Valéry, Montpellier, France, ${ }^{486}$ TUM School of Life Sciences Weihenstephan, Technical University of Munich, Freising, Germany, ${ }^{487}$ School of Life Science and Engineering, Southwest University of Science and Technology, Mianyang, China, ${ }^{488}$ Department of Crop and Forest Sciences \& Agrotecnio Center, Universitat de Lleida, Lleida, Spain, ${ }^{489}$ Universidade Federal do Acre, Rio Branco, AC, Brazil, ${ }^{490}$ Manaaki Whenua-Landcare Research, Lincoln, New Zealand, ${ }^{491}$ Freie Universität Berlin, Berlin, Germany, ${ }^{492}$ The University of Western Australia, Crawley, WA, Australia, ${ }^{493}$ Centre for Ecological Research and Forestry Applications (CREAF), Cerdanyola del Vallès, Spain, ${ }^{494}$ Ecology and Biodiversity, Vrije Universiteit Brussel, Brussels, Belgium, ${ }^{495}$ Laboratory of Wood Biology and Xylarium, Royal Museum for Central-Africa (RMCA), Tervuren, Belgium, ${ }^{496}$ Department of Forestry, Mississippi State University, Starkville, MS, USA, ${ }^{497}$ School of Biological Sciences, University of Southampton, Southampton, UK, ${ }^{498}$ Aquatic Ecology and Environmental Biology, Radboud University Nijmegen, Nijmegen, The Netherlands, ${ }^{499}$ School of Forestry \& Environmental Studies, Yale University, New Haven, CT, USA, ${ }^{500}$ Programa de pós-graduação em Ecologia, Universidade Federal do Rio Grande do Sul, Porto Alegre, RS, Brasil, ${ }^{501}$ Environmental and Climate Sciences Department, Brookhaven National Laboratory, NY, Upton, USA, ${ }^{502}$ Department of Biological Sciences, East Stroudsburg University, East Stroudsburg, PA, USA, ${ }^{503}$ Forest Research Group, INDEHESA, 
University of Extremadura, Plasencia, Spain, ${ }^{504}$ Physiological Diversity, Helmholtz Centre for Environmental Research (UFZ), Leipzig, Germany, ${ }^{505}$ Laboratorio Nacional de Ciencias de la Sostenibilidad, Instituto de Ecología, Universidad Nacional Autónoma de México, Ciudad Universitaria, Mexico City, Mexico, ${ }^{506}$ School of Environmental Sciences, University of Guelph, Guelph, ON, Canada, ${ }^{507}$ Remote Sensing Laboratories, Department of Geography, University of Zurich, Zurich, Switzerland, ${ }^{508}$ Research Unit Community Ecology, Swiss Federal Institute for Forest, Snow and Landscape Research WSL, Birmensdorf, Switzerland, ${ }^{509}$ Department of Research and Geoinformation, Swiss National Park, Chastè Planta-Wildenberg, Zernez, Switzerland, ${ }^{510}$ Centre for Ecology \& Hydrology (CEH), Wallingford, Oxfordshire, UK, ${ }^{511}$ Natural Resources Canada, Canadian Forest Service, Québec, QC, Canada, ${ }^{512}$ Departamento de Dinamica y Gestion Forestal, INIA-CIFOR, Madrid, Spain, ${ }^{513}$ Sustainable Forest Management Research Institute, University of Valladolid-INIA, Madrid, Spain, ${ }^{514}$ Department of Ecology and Evolution, University of Lausanne, Lausanne, Switzerland, ${ }^{515}$ Norwegian Institute for Nature Research, Trondheim, Norway, ${ }^{516}$ Departamento de Biología, Universidad Nacional de Colombia, Bogota, Colombia, ${ }^{517}$ Oxford University, Oxford, UK, ${ }^{518}$ Instituto de Recursos Naturales y Agrobiología de Salamanca (IRNASA-CSIC), Salamanca, Spain, ${ }^{519}$ Centre for Functional Ecology, Departamento de Ciências da Vida, Universidade de Coimbra, Coimbra, Portugal, ${ }^{520}$ Algoma University, Sault Ste. Marie, ON, Canada, ${ }^{521}$ Department of Geography, University of Zurich, Zürich, Switzerland, ${ }^{522}$ Senckenberg Biodiversität und Klima Forschungszentrum (SBiK-F), Frankfurt, Germany, ${ }^{523}$ Palmengarten der Stadt Frankfurt am Main, Frankfurt, Germany, ${ }^{524}$ Entomology III, Senckenberg Research Institute and Natural History Museum, Frankfurt am Main, Germany, ${ }^{525}$ Julius-von-Sachs-Institute for Biological Sciences, Chair of Ecophysiology and Vegetation Ecology, University of Wuerzburg, Wuerzburg, Germany, ${ }^{526}$ Herencia, Santa Cruz, Bolivia, ${ }^{527}$ Department of Earth and Environmental Sciences, University of Manchester, Manchester, UK, ${ }^{528}$ South African National Biodiversity Institute, Pretoria, South Africa, ${ }^{529}$ Federal University of Pernambuco, Recife, PE, Brazil, ${ }^{530}$ Sharplex Services, Edgecomb, ME, USA, ${ }^{531}$ Komarov Botanical Institute RAS, St. Petersburg, Russia, ${ }^{532}$ Research Institute for Humanity and Nature, Kyoto, Japan, ${ }^{533}$ Center for Southeast Asian Studies, Kyoto University, Kyoto, Japan, ${ }^{534}$ Université de Sherbrooke, Sherbrooke, QC, Canada, ${ }^{535}$ Department of Biology, University of Regina, Regina, SK, Canada, ${ }^{536}$ Technische Universität IImenau, IImenau, Germany, ${ }^{537}$ Centre for Applied Ecology "Professor Baeta Neves" (CEABN), School of Agriculture, University of Lisbon, Lisbon, Portugal, ${ }^{538}$ Department of Biology, Federal University of Lavras, Lavras, MG, Brazil, ${ }^{539}$ Department Land, Environment, Agriculture and Forestry, Università degli Studi di Padova, Padua, Italy, ${ }^{540}$ Department of Landscape Architecture, Planning and Management, Swedish University of Agricultural Sciences, Alnarp, Sweden, ${ }^{541}$ Gothenburg Botanical Garden, Gothenburg, Sweden, ${ }^{542}$ Gothenburg Global Biodiversity Centre, Gothenburg, Sweden, ${ }^{543}$ Texas Tech University, Lubbock, TX, USA, ${ }^{544}$ Forest Sciences Centre, Faculty of Forestry and Conservation Science, University of British Columbia, Vancouver, BC, Canada, ${ }^{545}$ Florida Museum of Natural History, University of Florida, Gainesville, FL, USA, ${ }^{546}$ Department of Earth and Environmental Sciences, KU Leuven, Leuven, Belgium, ${ }^{547}$ Archbold Biological Station, Venus, FL, USA, ${ }^{548}$ Embrapa Recursos Genéticos e Biotecnologia, Brasilia, DF, Brazil, ${ }^{549}$ Institute of Environmental Sciences, Leiden University, Leiden, The Netherlands, 550 Departamento de Ecologia, Universidade Federal do Rio Grande do Norte, Natal, RN, Brazil, ${ }^{551}$ Department of Evolution, Ecology, and Organismal Biology, University of California Riverside, Riverside, CA, USA, ${ }^{552}$ Department of Geography, Planning and Recreation, Northern Arizona University, Flagstaff, AZ, USA, ${ }^{553}$ Department of Ecology, Swedish University of Agricultural Sciences, Uppsala, Sweden, ${ }^{554}$ Forest Ecology and Forest Management Group, Wageningen University, The Netherlands, ${ }^{555}$ Institute of Lowland Forestry and Environment, University of Novi Sad, Novi Sad, Serbia, ${ }^{556}$ Stockholm University, Stockholm, Sweden, ${ }^{557}$ Instituto de Investigaciones en Biodiversidad y Medioambiente-CONICET, Universidad Nacional del Comahue, Bariloche, Argentina, ${ }^{558}$ Institute of Botany, Plant Science and Biodiversity Center, Slovak Academy of Sciences, Bratislava, Slovakia, ${ }^{559}$ Department of Ecology and General Biology, Faculty of Ecology and Environmental Sciences, Technical University in Zvolen, Zvolen, Slovakia, ${ }^{560}$ Department of Ecosystem Biology, Faculty of Science, University of South Bohemia, Ceske Budejovice, Czech Republic, ${ }^{561}$ Faculty of Forestry and Wood Sciences, Czech University of Life Sciences, Prague, Czech Republic, ${ }^{562}$ Department of Biology, University of Maryland, College Park, MD, USA, ${ }^{563}$ Departamento de Botânica, Universidade Federal de Pernambuco, Recife, PE, Brazil, ${ }^{564}$ Teshio Experimental Forest, Hokkaido University, Horonobe, Japan, ${ }^{565}$ Departamento de Ecología Funcional y Evolutiva, Estación Experimental de Zonas Áridas (CSIC), La Cañada de San Urbano, Spain, ${ }^{566}$ CIRAD-UMR SELMET-PZZS, Dakar, Senegal, ${ }^{567}$ Department of Biology, Hacettepe University, Ankara, Turkey, ${ }^{568}$ Environmental Sciences, Copernicus Institute of Sustainable Development, Utrecht University, Utrecht, The Netherlands, ${ }^{569}$ Centre for African Conservation Ecology, Nelson Mandela University, Port Elizabeth, South Africa, ${ }^{570}$ Natural Resources Canada, Canadian Wood Fibre Centre, Quebec, QC, Canada, ${ }^{571}$ Rubenstein School of Environment and Natural Resources, University of Vermont, Burlington, VT, USA, ${ }^{572}$ Bioversity International, Lima, Peru, ${ }^{573}$ Department of Animal and Plant Sciences, University of Sheffield, Sheffield, UK, ${ }^{574}$ Centre for Rainforest Studies, The School for Field Studies, Yungaburra, Qld, Australia, ${ }^{575}$ Department of Life Sciences, Imperial College London, Silwood Park, Ascot, UK, ${ }^{576}$ MTA-DE Lendület Functional and Restoration Ecology Research Group, Debrecen, Hungary, ${ }^{577}$ Department of Ecology, University of Debrecen, Debrecen, Hungary, ${ }^{578}$ Department of Soil and Plant Sciences, University of Delaware, Newark, DE, USA, ${ }^{579}$ INRA - Université ClermontAuvergne, UMR PIAF, Clermont-Ferrand, France, ${ }^{580} \mathrm{MTA}-\mathrm{TKI}$ Biodiversity and Ecosystem Services Research Group, Debrecen, Hungary, ${ }^{581}$ Department of Conservation Ecology and Entomology, Stellenbosch University, Matieland, South Africa, ${ }^{582}$ Illinois Natural 
History Survey, Prairie Research Institute, University of Illinois, Champaign, IL, USA, ${ }^{583}$ Unit for Modelling of Climate and Biogeochemical Cycles, UR-SPHERES, University of Liège, Liège, Belgique, ${ }^{584}$ Institute of Agricultural Sciences, Department of Environmental Systems Science, ETH Zurich, Zurich, Switzerland, ${ }^{585}$ School of Biological Sciences, The University of Western Australia, Crawley, WA, Australia, ${ }^{586}$ School of Biology, Department of Botany, Aristotle University of Thessaloniki, Greece, ${ }^{587} \mathrm{CIRAD}$, UMR EcoFoG (Agroparistech, CNRS, INRA, Université des Antilles, Université de la Guyane), Kourou, France, ${ }^{588}$ Osaka Natural History Center, Osaka, Japan, ${ }^{589}$ Ural State Forest Engineering University, Ekaterinburg, Russia, ${ }^{590}$ Botanical Garden of Ural Branch of Russian Academy of Sciences, Ekaterinburg, Russia, ${ }^{591}$ University of New Hampshire, Durham, NH, USA, ${ }^{592}$ Department of Biology, Faculty of Science, Ferdowsi University of Mashhad, Mashhad, Iran, ${ }^{593}$ Department of Biological Sciences, University of Calgary, Calgary, AB, Canada, ${ }^{594}$ College Environmental Studies, Yale University, New Haven, CT, USA, ${ }^{595}$ Department of Biological Sciences, National University of Singapore, Singapore, Singapore, ${ }^{596}$ Smithsonian Tropical Research Institute, Panama City, Panama, ${ }^{597}$ School of Geosciences, Edinburgh University, Edinburgh, UK, ${ }^{598}$ Systematic Botany and Functional Biodiversity, Institute of Biology, Leipzig University, Leipzig, Germany, ${ }^{599}$ Institute for Global Ecology, Florida Institute of Technology, Melbourne, FL, USA, ${ }^{600}$ Department of Biology, University of Konstanz, Konstanz, Germany, ${ }^{601}$ Zhejiang Provincial Key Laboratory of Plant Evolutionary Ecology and Conservation, Taizhou University, Taizhou, China, ${ }^{602}$ Institute of Geography, University of Erlangen-Nuremberg, Erlangen, Germany, ${ }^{603}$ Department of Environmental Biology, Sapienza University of Rome, Rome, Italy, ${ }^{604}$ Laboratorio de invasiones Biológicas, Universidad de Concepcion, Concepcion, Chile, ${ }^{605}$ Institute of Biodiversity and Ecosystem Research, Bulgarian Academy of Sciences, Sofia, Bulgaria, ${ }^{606}$ School of Biological Sciences and School of Agriculture and Environment, The University of Western Australia, Crawley, WA, Australia, ${ }^{607}$ Department of Environment, Forest \& Nature Lab, Ghent University, Gontrode-Melle, Belgium, ${ }^{608}$ Museu Paraense Emilio Goeldi, Belém, PA, Brazil, ${ }^{609}$ Departamento de Ciencias de la Vida, Universidad de las Fuerzas Armadas (ESPE),
Sangolquí, Ecuador, ${ }^{610}$ Department of Botany, Goa University, Goa, India, ${ }^{611}$ Department of Ecology and Environmental Sciences, Pondicherry University, Puducherry, India, ${ }^{612}$ Carl von Ossietzky University of Oldenburg, Oldenburg, Germany, ${ }^{613}$ Zoology Department, Edward Grey Institute, Oxford University, Oxford, UK, ${ }^{614}$ Department of Zoology, Cambridge University, Cambridge Conservation Initiative, Cambridge, UK, ${ }^{615}$ Ministry of Education Key Laboratory for Earth System Modeling, Department of Earth System Science, Tsinghua University, Beijing, China, ${ }^{616}$ Institute of Desertification Studies, Chinese Academy of Forestry, Beijing, China, ${ }^{617}$ Institute of Geography, Fujian Normal University, Fuzhou, China, ${ }^{618}$ Department of Landscape Architecture, University of Sheffield, Sheffield, UK, ${ }^{619}$ Department of Biology, Colgate University, Hamilton, NY, USA, ${ }^{620}$ Ecological Sciences, Vrije Universiteit Amsterdam, Amsterdam, The Netherlands, ${ }^{621}$ Maritime and Science Technology Academy, Miami, FL, USA, ${ }^{622}$ Free University of Bozen-Bolzano, Bolzano, Italy, ${ }^{623}$ University of Winnipeg, Winnipeg, Manitoba, Canada, ${ }^{624}$ The James Hutton Institute, Dundee, UK, ${ }^{625}$ King Saud University, Riyadh, Saudi Arabia, ${ }^{626}$ University of California - Irvine, Irvine, CA, USA, ${ }^{627}$ Southwest Biological Science Center, U. S. Geological Survey, Moab, UT, USA, ${ }^{628}$ Department of Biology, Duke University, Durham, NC, USA, ${ }^{629}$ NSW Department of Primary Industries, Parramatta, NSW, Australia, ${ }^{630}$ Hiroshima University, Higashi-Hiroshima, Japan, ${ }^{631}$ Graduate School of Life and Environmental Sciences, University of Tsukuba, Tsukuba, Japan, ${ }^{632}$ SUNY-College of Environmental Science and Forestry, Syracuse, NY, USA, ${ }^{633}$ Centre d'Ecologie et des Sciences de la Conservation (CESCO), Muséum National d'Histoire Naturelle, Centre National de la Recherche Scientifique, Sorbonne-Université, Paris, France, ${ }^{634}$ Laboratório de Ecologia Vegetal (LEVEG), Porto Alegre, RS, Brazil, ${ }^{635}$ Biological Sciences, George Washington University, Washington, DC, USA, ${ }^{636}$ National Taiwan University, Taipei, Taiwan, ${ }^{637}$ College of Life Sciences, Zhejiang University, Hangzhou, China, ${ }^{638}$ Forestry College, Beijing Forestry University, Beijing, China, ${ }^{639}$ Institut National Polytechnique Félix Houphouët-Boigny (INP-HB), Yamoussoukro, Côte d'Ivoire, ${ }^{640}$ Institute for Biology and Environmental Sciences, University Oldenburg, Oldenburg, Germany 
TAB LE A1 Datasets contributed to the TRY plant trait database. Sorted by custodian surname. ID: Dataset ID in the TRY database, Name, Custodian, TRY version to which the dataset was submitted (in parentheses first submission), availability of the dataset (status 1.10.2019), reference

\begin{tabular}{|c|c|c|c|c|c|}
\hline ID & Dataset name & Custodian & Version & Availability & Reference \\
\hline 429 & TraitDunes & Alicia T.R. Acosta & 5 & Restricted & Unpublished \\
\hline 285 & $\begin{array}{l}\text { Functional traits explaining variation in plant life } \\
\text { history strategies }\end{array}$ & Peter Adler & 4 & Public & Adler et al. (2013) \\
\hline 582 & Guisane2080 & Cécile Albert & 6 & Restricted & Albert et al. (2010) \\
\hline 583 & Ecophy & Cécile Albert & 6 & Restricted & Albert et al. (2011) \\
\hline 268 & $\begin{array}{l}\text { Seed Longevity of European Early Successional } \\
\text { Species }\end{array}$ & Harald Albrecht & 3 & Public & Unpublished \\
\hline 559 & Haean_South_Korea_Traits & Hamada Ali & 6 & Public & $\begin{array}{l}\text { Ali, Reineking, and Münkemüller } \\
\text { (2017) }\end{array}$ \\
\hline 150 & French Weeds Trait Database & Bernard Amiaud & 3 & Public & Unpublished \\
\hline 376 & $\begin{array}{l}\text { Biomass allocation in beech and spruce } \\
\text { seedlings }\end{array}$ & Christian Ammer & 4 & Public & $\begin{array}{l}\text { Schall, Lödige, Beck, and Ammer } \\
\text { (2012) }\end{array}$ \\
\hline 100 & Plant Traits in Pollution Gradients Database & Madhur Anand & 2 & Restricted & Unpublished \\
\hline 624 & $\begin{array}{l}\text { CPCRW Carbon Dynamics Along Permafrost } \\
\text { Gradient: Specific Leaf Area of Alder and } \\
\text { Spruce }\end{array}$ & Carolyn Anderson & 6 & Public & Unpublished \\
\hline 76 & $\begin{array}{l}\text { European Mountain Meadows Plant Traits } \\
\text { Database }\end{array}$ & Michael Bahn & 1 & Restricted & Bahn et al. (1999) \\
\hline 101 & Photosynthesis Traits Database & Dennis Baldocchi & 2 & Public & Xu and Baldocchi (2003) \\
\hline 154 & $\begin{array}{l}\text { Leaf Photosynthesis and Nitrogen at Oak Ridge } \\
\text { Dataset }\end{array}$ & Dennis Baldocchi & 2 & Public & $\begin{array}{l}\text { Wilson, Baldocchi, and Hanson } \\
(2000)\end{array}$ \\
\hline 269 & The Bridge Database & Chris Baraloto & 3 & Public & $\begin{array}{l}\text { Baraloto, Timothy Paine, Poorter, } \\
\text { et al. (2010) }\end{array}$ \\
\hline 422 & $\begin{array}{l}\text { Hawaii native and non-indigenous species. Traits } \\
\text { and environment }\end{array}$ & Zdravko Baruch & 5 & Public & Baruch and Goldstein (1999) \\
\hline 576 & Bauerle Vcmax and Jmax data & William Bauerle & 6 & Restricted & Bauerle et al. (2012) \\
\hline 502 & Yangambi arboretum & Marijn Bauters & 6 & Restricted & Bauters et al. (2015) \\
\hline 504 & Djolu & Marijn Bauters & 6 & Restricted & Bauters et al. (2019) \\
\hline 505 & Nyungwe_Rwanda & Marijn Bauters & 6 & Restricted & Bauters et al. (2017) \\
\hline 654 & $\begin{array}{l}\text { Plant height of Mediterranean herb layer com- } \\
\text { munities, Sardinia, Italy }\end{array}$ & Erika Bazzato & 6 & Restricted & Unpublished \\
\hline 277 & $\begin{array}{l}\text { UV-B Radiation Sensitivity of Hieracium } \\
\text { Pilosella }\end{array}$ & $\begin{array}{l}\text { Michael } \\
\text { Beckmann }\end{array}$ & 3 & Public & $\begin{array}{l}\text { Beckmann, Hock, Bruelheide, } \\
\text { and Erfmeier (2012) }\end{array}$ \\
\hline
\end{tabular}


TABLE A1 (Continued)

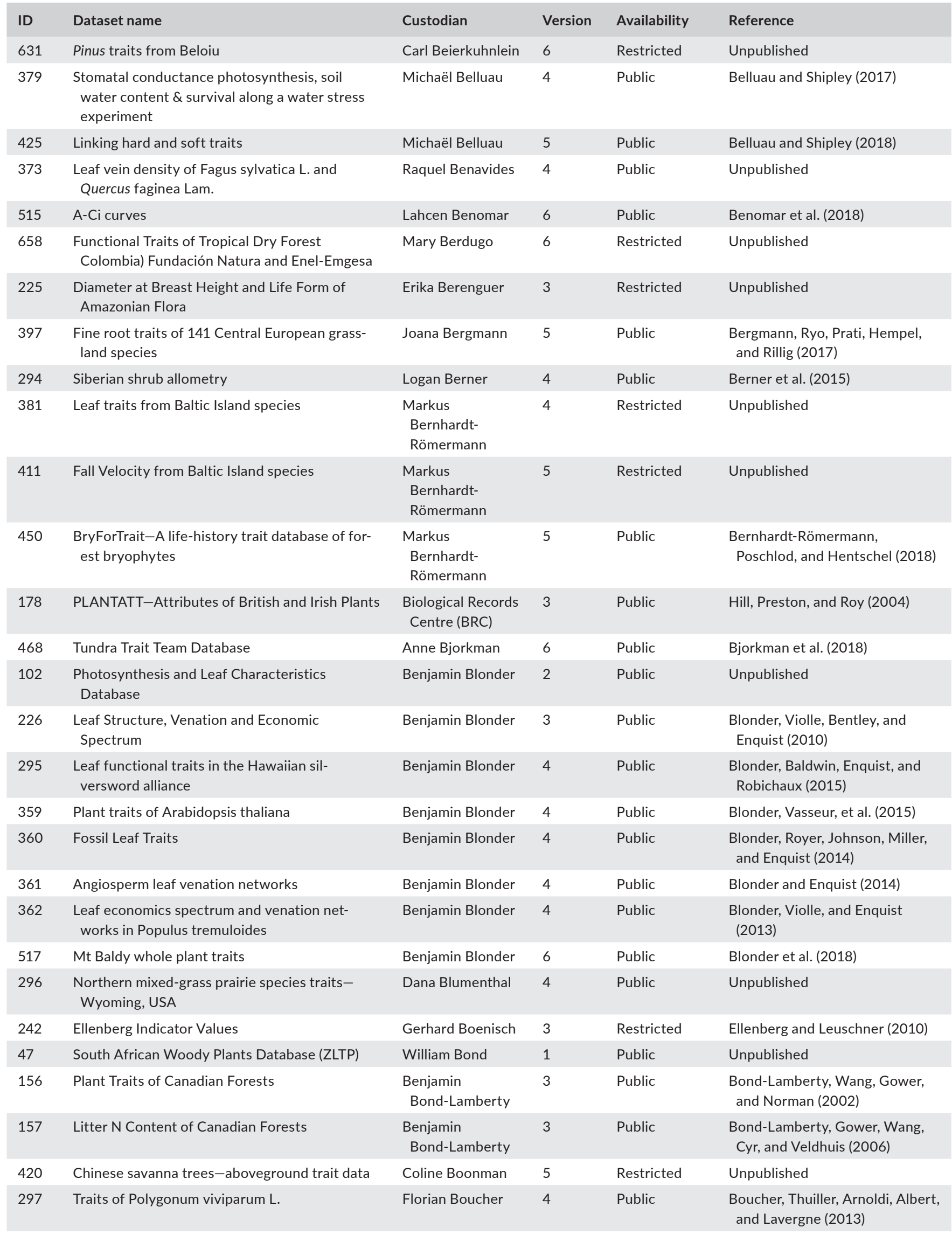


TABLE A1 (Continued)

\begin{tabular}{|c|c|c|c|c|c|}
\hline ID & Dataset name & Custodian & Version & Availability & Reference \\
\hline 636 & La Selva FT Data & Vanessa Boukili & 6 & Public & Boukili and Chazdon (2017) \\
\hline 421 & LECA-Traits of the European Alpine Flora & Louise Boulangeat & 5 & Public & Unpublished \\
\hline 165 & Leaf Traits From Madagascar & Kerry Brown & 3 & Restricted & Brown et al. (2013) \\
\hline 428 & $\begin{array}{l}\text { Trait and biomass data } 2014 \text { and } 2015 \text { of the } \\
\text { BE_LOW project }\end{array}$ & Helge Bruelheide & 5 & Public & Herz et al. (2017) \\
\hline 298 & Plant traits from Greby, Oeland, Sweden & $\begin{array}{l}\text { Hans Henrik } \\
\text { Bruun }\end{array}$ & 4 & Restricted & $\begin{array}{l}\text { Baastrup-Spohr, Sand-Jensen, } \\
\text { Nicolajsen, and Bruun (2015) }\end{array}$ \\
\hline 545 & $\begin{array}{l}\text { Dataset on reproductive traits of Scandinavian } \\
\text { alpine plants }\end{array}$ & $\begin{array}{l}\text { Hans Henrik } \\
\text { Bruun }\end{array}$ & 6 & Public & Bruun (2019) \\
\hline 416 & Garmisch-Partenkirchen elevational gradients & $\begin{array}{l}\text { Solveig Franziska } \\
\text { Bucher }\end{array}$ & 5 & Restricted & Bucher et al. (2016) \\
\hline 7 & Cedar Creek Plant Physiology Database & Daniel Bunker & 1 & Restricted & Unpublished \\
\hline 585 & Arable weed trait data set & Jana Bürger & 6 & Restricted & Unpublished \\
\hline 158 & Plant Traits from Circeo National Park, Italy & Sabina Burrascano & 3 & Public & Burrascano et al. (2015) \\
\hline 159 & Traits of US Desert Woody Plant Species & $\begin{array}{l}\text { Bradley } \\
\text { Butterfield }\end{array}$ & 3 & Public & Butterfield and Briggs (2010) \\
\hline 160 & SLA and LDMC for Canadian Wetland Species & Chaeho Byun & 3 & Restricted & $\begin{array}{l}\text { Byun, de Blois, and Brisson } \\
\text { (2012) }\end{array}$ \\
\hline 161 & Leaf Traits in Central Apennines Beech Forests & $\begin{array}{l}\text { Giandiego } \\
\text { Campetella }\end{array}$ & 3 & Public & Campetella et al. (2011) \\
\hline 220 & $\begin{array}{l}\text { Leaf Traits in Italian Central Apennines Beech } \\
\text { Forests }\end{array}$ & $\begin{array}{l}\text { Giandiego } \\
\text { Campetella }\end{array}$ & 3 & Public & Campetella et al. (2011) \\
\hline 406 & $\begin{array}{l}\text { Whole plant traits and leaf traits of four grass- } \\
\text { land species in Central Apennines (Italy) }\end{array}$ & $\begin{array}{l}\text { Giandiego } \\
\text { Campetella }\end{array}$ & 5 & Restricted & Wellstein et al. (2013) \\
\hline 600 & Bay of Biscay dunes & $\begin{array}{l}\text { Juan Antonio } \\
\text { Campos }\end{array}$ & 6 & Restricted & $\begin{array}{l}\text { Torca, Campos, and Herrera } \\
\text { (2019) }\end{array}$ \\
\hline 503 & $\begin{array}{l}\text { Leaf and whole plant traits of Val Cervara old } \\
\text { growth beech forest (Central Apennine, Italy) }\end{array}$ & Roberto Canullo & 6 & Restricted & Unpublished \\
\hline 649 & $\begin{array}{l}\text { Alpine tundra plants-Effects of climate } \\
\text { warming on traits of species in mid-latitude } \\
\text { snowbeds }\end{array}$ & $\begin{array}{l}\text { Michele } \\
\text { Carbognani }\end{array}$ & 6 & Public & Unpublished \\
\hline 595 & UFPR Atlantic Forest Tree Traits & Marcos Carlucci & 6 & Restricted & Unpublished \\
\hline 670 & Fabio Carvalho lowland fen peatland & Fabio Carvalho & 6 & Restricted & $\begin{array}{l}\text { Carvalho, Brown, Waller, and } \\
\text { Boom (2019); Carvalho, Brown, } \\
\text { Waller, Bunting, et al. (2019) }\end{array}$ \\
\hline 299 & $\begin{array}{l}\text { Traits related to riparian plant invasion in South } \\
\text { East Australia }\end{array}$ & Jane Catford & 4 & Restricted & $\begin{array}{l}\text { Catford, Morris, Vesk, Gippel, } \\
\text { and Downes (2014) }\end{array}$ \\
\hline
\end{tabular}


TABLE A1 (Continued)

\begin{tabular}{|c|c|c|c|c|c|}
\hline ID & Dataset name & Custodian & Version & Availability & Reference \\
\hline 227 & Leaf Structure and Economics Spectrum & $\begin{array}{l}\text { Bruno E. L. } \\
\text { Cerabolini }\end{array}$ & $3(4)$ & Restricted & $\begin{array}{l}\text { Pierce, Ceriani, De Andreis, } \\
\text { Luzzaro, and Cerabolini (2007) }\end{array}$ \\
\hline 229 & Hydrophytes Traits Database & $\begin{array}{l}\text { Bruno E. L. } \\
\text { Cerabolini }\end{array}$ & $3(4)$ & Restricted & $\begin{array}{l}\text { Pierce, Brusa, Sartori, and } \\
\text { Cerabolini (2012) }\end{array}$ \\
\hline 371 & Olive Lawn Orchid Trait Database (OLO) & $\begin{array}{l}\text { Bruno E. L. } \\
\text { Cerabolini }\end{array}$ & 4 & Restricted & $\begin{array}{l}\text { Pierce, Vagge, Brusa, and } \\
\text { Cerabolini (2014) }\end{array}$ \\
\hline 300 & $\begin{array}{l}\text { Leaf traits from the Loess Plateau region of } \\
\text { northern Shaanxi in China }\end{array}$ & Yongfu Chai & 4 & Restricted & Unpublished \\
\hline 120 & Tropical Respiration Database & Jeffrey Chambers & 2 & Public & Chambers et al. (2004) \\
\hline 73 & Tundra Plant Traits Database & F Stuart Chapin III & 1 & Public & Unpublished \\
\hline 501 & Leaf traits of beech forest understory species & Stefano Chelli & 6 & Restricted & Unpublished \\
\hline 611 & Temperate tree species in New Jersey USA & Anping Chen & 6 & Public & $\begin{array}{l}\text { Chen, Lichstein, Osnas, and } \\
\text { Pacala (2014) }\end{array}$ \\
\hline 498 & Fruit type, fruit dimension and flowering time & Si-Chong Chen & 6 & Public & $\begin{array}{l}\text { Chen, Cornwell, Zhang, and } \\
\text { Moles (2017) }\end{array}$ \\
\hline 349 & Mediterranean psammophytes & Daniela Ciccarelli & 4 & Public & Ciccarelli (2015) \\
\hline 394 & $\begin{array}{l}\text { Great Basin sagebrush seedlings-greenhouse } \\
\text { experiment }\end{array}$ & Courtney Collins & 5 & Public & Unpublished \\
\hline 1 & Abisko and Sheffield Database & $\begin{array}{l}\text { Johannes } \\
\text { Cornelissen }\end{array}$ & 1 & Public & Cornelissen et al. (2004) \\
\hline 37 & Sheffield Database & $\begin{array}{l}\text { Johannes } \\
\text { Cornelissen }\end{array}$ & 1 & Public & $\begin{array}{l}\text { Cornelissen, Diez, and Hunt } \\
\text { (1996) }\end{array}$ \\
\hline 72 & Sheffield and Spain Woody Database & $\begin{array}{l}\text { Johannes } \\
\text { Cornelissen }\end{array}$ & 1 & Public & $\begin{array}{l}\text { Castro-Díez, Puyravaud, } \\
\text { Cornelissen, and Villar-Salvador } \\
\text { (1998) }\end{array}$ \\
\hline 121 & Fern Spore Mass Database & $\begin{array}{l}\text { Johannes } \\
\text { Cornelissen }\end{array}$ & 2 & Public & Unpublished \\
\hline 55 & $\begin{array}{l}\text { Jasper Ridge Californian Woody Plants } \\
\text { Database }\end{array}$ & Will Cornwell & 1 & Public & $\begin{array}{l}\text { Preston, Cornwell, and DeNoyer } \\
\text { (2006) }\end{array}$ \\
\hline 89 & ArtDeco Database & Will Cornwell & $1(2)$ & Restricted & Cornwell et al. (2008) \\
\hline 430 & A Global Dataset of Leaf $\Delta 13 C$ Data & Will Cornwell & 5 & Public & Cornwell et al. (2018) \\
\hline 280 & Global Woodiness Database & William Cornwell & 3 & Public & Zanne et al. (2014) \\
\hline
\end{tabular}


TABLE A1 (Continued)

\begin{tabular}{|c|c|c|c|c|c|}
\hline ID & Dataset name & Custodian & Version & Availability & Reference \\
\hline 10 & Roots Of the World (ROW) Database & Joseph Craine & 1 & Public & $\begin{array}{l}\text { Craine, Lee, Bond, Williams, and } \\
\text { Johnson (2005) }\end{array}$ \\
\hline 163 & $\begin{array}{l}\text { Plant Traits for Grassland Species (Konza Prairie, } \\
\text { Kansas, USA) }\end{array}$ & Joseph Craine & 3 & Public & Craine et al. (2011) \\
\hline 293 & Jasper Ridge leaf chemistry data & Kyla Dahlin & 4 & Public & Dahlin, Asner, and Field (2013) \\
\hline 164 & Italian Alps Plant Traits Database & Matteo Dainese & 3 & Public & Dainese and Bragazza (2012) \\
\hline 346 & $\begin{array}{l}\text { Leaf traits of Dipterocarpus alatus Roxb. ex. G. } \\
\text { Don }\end{array}$ & Anh Tuan Dang-Le & 4 & Public & $\begin{array}{l}\text { Dang-Le, Edelin, and Le-Cong } \\
\text { (2013) }\end{array}$ \\
\hline 224 & $\begin{array}{l}\text { LBA-ECO CD-02 C and N Isotopes in Leaves and } \\
\text { Atmospheric } \mathrm{CO}_{2} \text {, Amazonas, Brazil }\end{array}$ & $\begin{array}{l}\text { Alessandro de } \\
\text { Araujo }\end{array}$ & 3 & Public & de Araujo et al. (2012) \\
\hline 643 & Lapalala grass trait data 2019 & Arend de Beer & 6 & Restricted & Unpublished \\
\hline 289 & Cabo de Gata-Níjar Natural Park & Angel de Frutos & 4 & Restricted & $\begin{array}{l}\text { de Frutos, Navarro, Pueyo, and } \\
\text { Alados (2015) }\end{array}$ \\
\hline 167 & Leaf N-Retention Database & Franciska de Vries & 3 & Public & de Vries and Bardgett (2016) \\
\hline 525 & $\begin{array}{l}\text { Arboretum Grosspoessna } 2014 \text { leaf chemical } \\
\text { and photosynthesis traits }\end{array}$ & Benjamin Dechant & 6 & Restricted & $\begin{array}{l}\text { Dechant, Cuntz, Vohland, Schulz, } \\
\text { and Doktor (2017) }\end{array}$ \\
\hline 542 & $\begin{array}{l}\text { Smilax auriculata nonstructural carbohydrates } \\
\text { under-ground }\end{array}$ & Milton Diaz & 6 & Restricted & Unpublished \\
\hline 86 & Sheffield-Iran-Spain Database & Sandra Díaz & 1 & Public & Díaz et al. (2004) \\
\hline 189 & Mycorrhiza Database & Ian Dickie & 3 & Public & $\begin{array}{l}\text { Koele, Dickie, Oleksyn, } \\
\text { Richardson, and Reich (2012) }\end{array}$ \\
\hline 231 & TROBIT West Africa & Tomas Domingues & 3 & Restricted & Domingues et al. (2010) \\
\hline 232 & LBA ECO CD02: Tapajos Leaf Water Potential & Tomas Domingues & 3 & Restricted & Almeida et al. (2001) \\
\hline 255 & $\begin{array}{l}\text { LBA ECO Tapajos: Leaf Characteristics and } \\
\text { Photosynthesis }\end{array}$ & Tomas Domingues & 3 & Restricted & $\begin{array}{l}\text { Domingues, Martinelli, and } \\
\text { Ehleringer (2007) }\end{array}$ \\
\hline 614 & Ausplot traits & Ning Dong & 6 & Public & Dong et al. (2017) \\
\hline 169 & $\begin{array}{l}\text { Traits for Submerged Species (Aquatic } \\
\text { Macrophytes) }\end{array}$ & Matthew Dunkle & 3 & Public & Unpublished \\
\hline 301 & $\begin{array}{l}\text { Specific leaf area responses to environmental } \\
\text { gradients through space and time }\end{array}$ & John Dwyer & 4 & Public & $\begin{array}{l}\text { Dwyer, Hobbs, and Mayfield } \\
(2014)\end{array}$ \\
\hline 467 & $\begin{array}{l}\text { Data on chlorophylls and carotenoids in plants } \\
\text { and lichens at the European Northeast of } \\
\text { Russia }\end{array}$ & Olga Dymova & 6 & Restricted & $\begin{array}{l}\text { Golovko, Dymova, Yatsco, and } \\
\text { Tabalenkova (2011) }\end{array}$ \\
\hline
\end{tabular}


TABLE A1 (Continued)

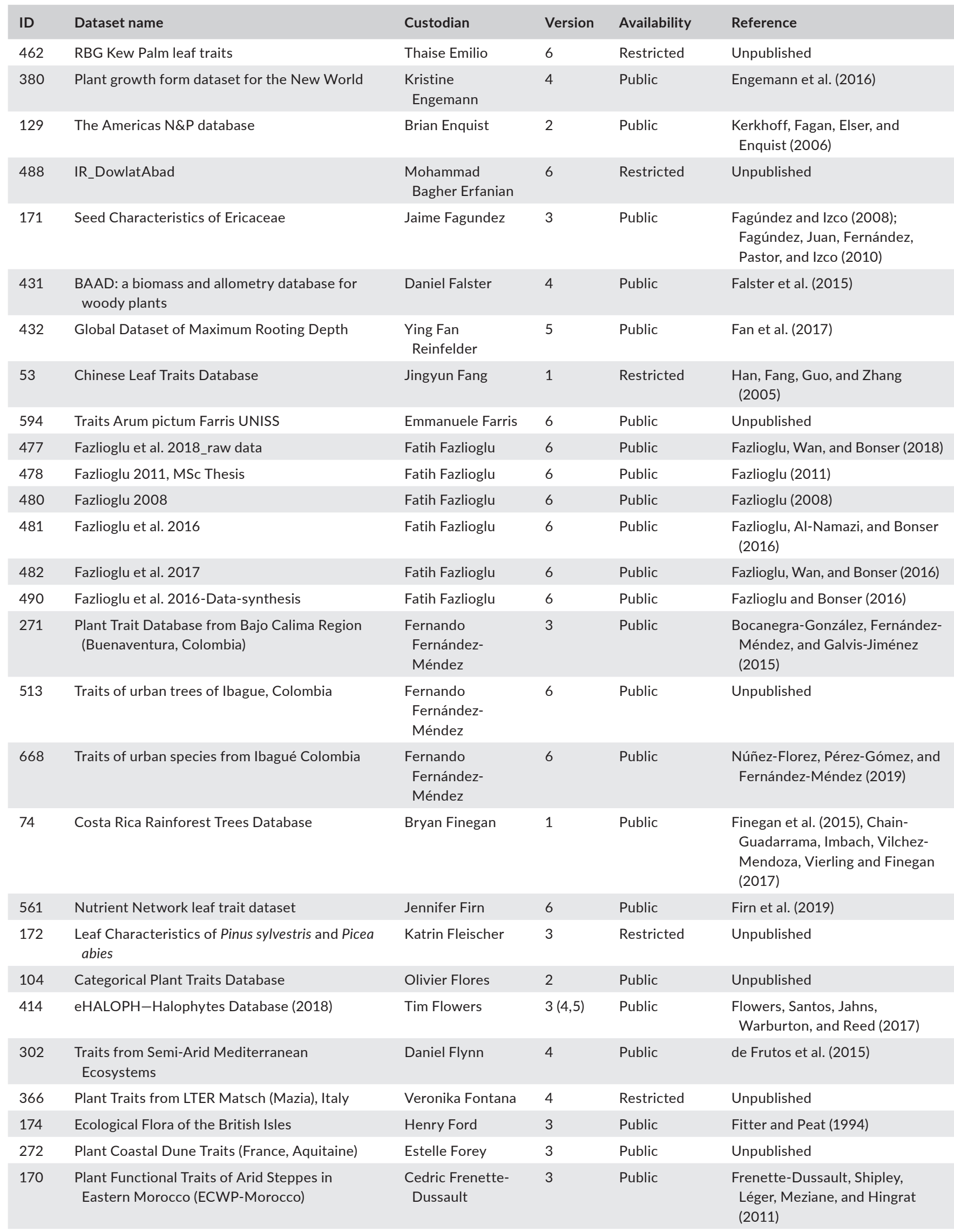


TABLE A1 (Continued)

\begin{tabular}{|c|c|c|c|c|c|}
\hline ID & Dataset name & Custodian & Version & Availability & Reference \\
\hline 507 & Freschet et al. 2018 & Gregoire Freschet & 6 & Public & Freschet et al. (2018) \\
\hline 511 & Freschet et al. 2015-Mount Hutt & Gregoire Freschet & 6 & Public & $\begin{array}{l}\text { Freschet, Kichenin, and Wardle } \\
\text { (2015) }\end{array}$ \\
\hline 661 & $\begin{array}{l}\text { Mediterranean Forests in Transition (MEDIT) } \\
\text { dataset }\end{array}$ & Nikolaos Fyllas & 6 & Restricted & Fyllas et al. (2017) \\
\hline 175 & $\begin{array}{l}\text { BASECO: a floristic and ecological database of } \\
\text { Mediterranean French flora }\end{array}$ & Sophie Gachet & 3 & Public & Gachet, Véla, and Tatoni (2005) \\
\hline 176 & Climbing plants trait dataset & Rachael Gallagher & 3 & Public & Gallagher and Leishman (2012) \\
\hline 177 & Litter Traits Dataset & $\begin{array}{l}\text { Pablo } \\
\text { García-Palacios }\end{array}$ & 3 & Public & $\begin{array}{l}\text { García-Palacios, Maestre, Kattge, } \\
\text { and Wall (2013) }\end{array}$ \\
\hline 45 & The VISTA Plant Trait Database & Eric Garnier & 1 & Restricted & Garnier et al. (2007) \\
\hline 383 & Species and trait shifts in Apennine grasslands & Eleonora Giarrizzo & 4 & Public & Giarrizzo et al. (2016) \\
\hline 664 & Khalil Prairie Plant Traits & David Gibson & 6 & Public & $\begin{array}{l}\text { Khalil, Gibson, Baer, and Willand } \\
\text { (2018) }\end{array}$ \\
\hline 382 & $\begin{array}{l}\text { Species able to reproduce after fire in a Brazilian } \\
\text { Savanna }\end{array}$ & Aelton B. Giroldo & 4 & Restricted & Giroldo (2016) \\
\hline 514 & Macquarie xylem leaf site hydraulics & Sean Gleason & 6 & Public & Unpublished \\
\hline 529 & $\begin{array}{l}\text { Diurnal and nocturnal gas exchange Quercus } \\
\text { spp. }\end{array}$ & Elena Granda & 6 & Restricted & Unpublished \\
\hline 530 & $\begin{array}{l}\text { Seasonal gas exchange photoperiod Quercus } \\
\text { spp. }\end{array}$ & Elena Granda & 6 & Restricted & Granda et al. (2020) \\
\hline 92 & PLANTSdata USDA & Walton Green & 1 & Public & Green (2009) \\
\hline 512 & Chromosome numbers of the Flora of Germany & Thomas Gregor & 6 & Public & Paule et al. (2017) \\
\hline 275 & $\begin{array}{l}\text { Plant Traits From Spanish Mediterranean } \\
\text { shrublands }\end{array}$ & Nicholas Gross & 3 & Public & Unpublished \\
\hline 460 & TRY Categorical Traits Dataset (update 2018) & Angela Guenther & 5 & Public & Unpublished \\
\hline 179 & $\begin{array}{l}\text { Leaf Gross Morphometrics Within one Species } \\
\text { in Relation to Latitude, Altitude and Time }\end{array}$ & Greg Guerin & 3 & Public & Guerin, Wen, and Lowe (2012) \\
\hline 123 & Virtual Forests Trait Database & $\begin{array}{l}\text { Alvaro G. } \\
\text { Gutierrez }\end{array}$ & 2 & Public & Gutiérrez and Huth (2012) \\
\hline 609 & SERC-PREMIS Leaf Trait Dataset & Lillie Haddock & 6 & Public & Unpublished \\
\hline 586 & Cedrus atlantica traits & Alain Hambuckers & 6 & Restricted & Unpublished \\
\hline 180 & Leaf Ash Content in China's Terrestrial Plants & Wenxuan Han & 3 & Public & Han et al. (2012) \\
\hline 181 & $\begin{array}{l}\text { Leaf Nitrogen and Phosphorus for China's } \\
\text { Terrestrial Plants }\end{array}$ & Wenxuan Han & 3 & Public & $\begin{array}{l}\text { Chen, Han, Tang, Tang, and Fang } \\
\text { (2013) }\end{array}$ \\
\hline 236 & Chinese Traits & Sandy Harrison & 3 & Public & Prentice et al. (2010) \\
\hline
\end{tabular}


TABLE A1 (Continued)

\begin{tabular}{|c|c|c|c|c|c|}
\hline ID & Dataset name & Custodian & Version & Availability & Reference \\
\hline 183 & Komati Leaf Trait Data & Wesley Hattingh & 3 & Restricted & Unpublished \\
\hline 541 & Rede Amazônia Sustentável & Joseph Hawes & 6 & Public & Unpublished \\
\hline 367 & $\begin{array}{l}\text { Tree species functional traits from Dinghushan } \\
\text { Biosphere Reserve, southern China }\end{array}$ & Pengcheng $\mathrm{He}$ & 4 & Public & Li et al. (2015) \\
\hline 669 & $\begin{array}{l}\text { Leaf Economics Traits of Woody Species in } \\
\text { Dinghushan Biosphere Reserve, Southern } \\
\text { China }\end{array}$ & Pengcheng $\mathrm{He}$ & 6 & Public & He et al. (2019) \\
\hline 434 & $\begin{array}{l}\text { Seed mass and nutrient concentration in } \\
\text { Grevillea and Hakea species }\end{array}$ & Tianhua He & 5 & Public & He, Fowler, and Causley (2015) \\
\hline 472 & $\begin{array}{l}\text { Traits data for plant species from Western } \\
\text { Australia }\end{array}$ & Tianhua He & 6 & Public & Unpublished \\
\hline 628 & Peel Forest New Zealand Sycamore dataset & Mason Heberling & 6 & Public & Heberling and Mason (2018) \\
\hline 634 & $\begin{array}{l}\text { Trillium Trail Forest Wildflower Carbon Gain } \\
\text { Phenology }\end{array}$ & Mason Heberling & 6 & Public & $\begin{array}{l}\text { Heberling, Cassidy, Fridley, and } \\
\text { Kalisz (2019) }\end{array}$ \\
\hline 546 & $\begin{array}{l}\text { Bark, Leaf and Root traits of tropical trees from } \\
\text { the semi-deciduous forests of TENE, West } \\
\text { Africa }\end{array}$ & Bruno Herault & 6 & Restricted & Unpublished \\
\hline 185 & cDNA Content of Carex & Andrew Hipp & 3 & Public & Chung, Hipp, and Roalson (2012) \\
\hline 671 & $\begin{array}{l}\text { Morton Arboretum Experimental Prairie traitset } \\
\text { 1, } 2019\end{array}$ & Andrew Hipp & 6 & Public & Hipp et al. (2018) \\
\hline 659 & $\begin{array}{l}\text { Sjöman-Hirons Leaf Turgor Loss with Osmotic } \\
\text { Potential at Full Turgor }\end{array}$ & Andrew Hirons & 6 & Restricted & $\begin{array}{l}\text { Sjöman, Hirons, and Bassuk } \\
\text { (2015) }\end{array}$ \\
\hline 509 & $\begin{array}{l}\text { Leaf functional traits for tropical saplings from } \\
\text { Jianfengling, Hainan Island, China }\end{array}$ & J. Aaron Hogan & 6 & Public & $\begin{array}{l}\text { Hogan, Valverde-Barrantes, } \\
\text { Ding, Xu, and Baraloto (2019) }\end{array}$ \\
\hline 306 & Plant traits from Costa Rica & Karen Holl & 4 & Public & Unpublished \\
\hline 291 & MARGINS-leaf traits database & Daniel Hornstein & 4 & Public & Unpublished \\
\hline 476 & $\begin{array}{l}\text { Leaf traits and litter properties in Dinghu moun- } \\
\text { tain, Guangdong province, China }\end{array}$ & Enqing Hou & 6 & Public & $\begin{array}{l}\text { Hou, Chen, McGroddy, and Wen } \\
\text { (2012) }\end{array}$ \\
\hline 287 & $\begin{array}{l}\text { Biomass allocation of Carex obnupta and Carex } \\
\text { stipata }\end{array}$ & Nate Hough-Snee & 4 & Public & $\begin{array}{l}\text { Hough-Snee, Nackley, Kim, and } \\
\text { Ewing (2015) }\end{array}$ \\
\hline 355 & Knautia arvensis; Mid-Norway & Knut Hovstad & 4 & Restricted & Unpublished \\
\hline 580 & Alpyr & Estela IIla & 6 & Restricted & Unpublished \\
\hline 551 & Coffee traits & Marney Isaac & 6 & Public & Isaac et al. (2017) \\
\hline 463 & Leaf Chlorophyll and Carotenoids Database & Leonid Ivanov & 6 & Restricted & Unpublished \\
\hline
\end{tabular}

(Continues) 
TABLE A1 (Continued)

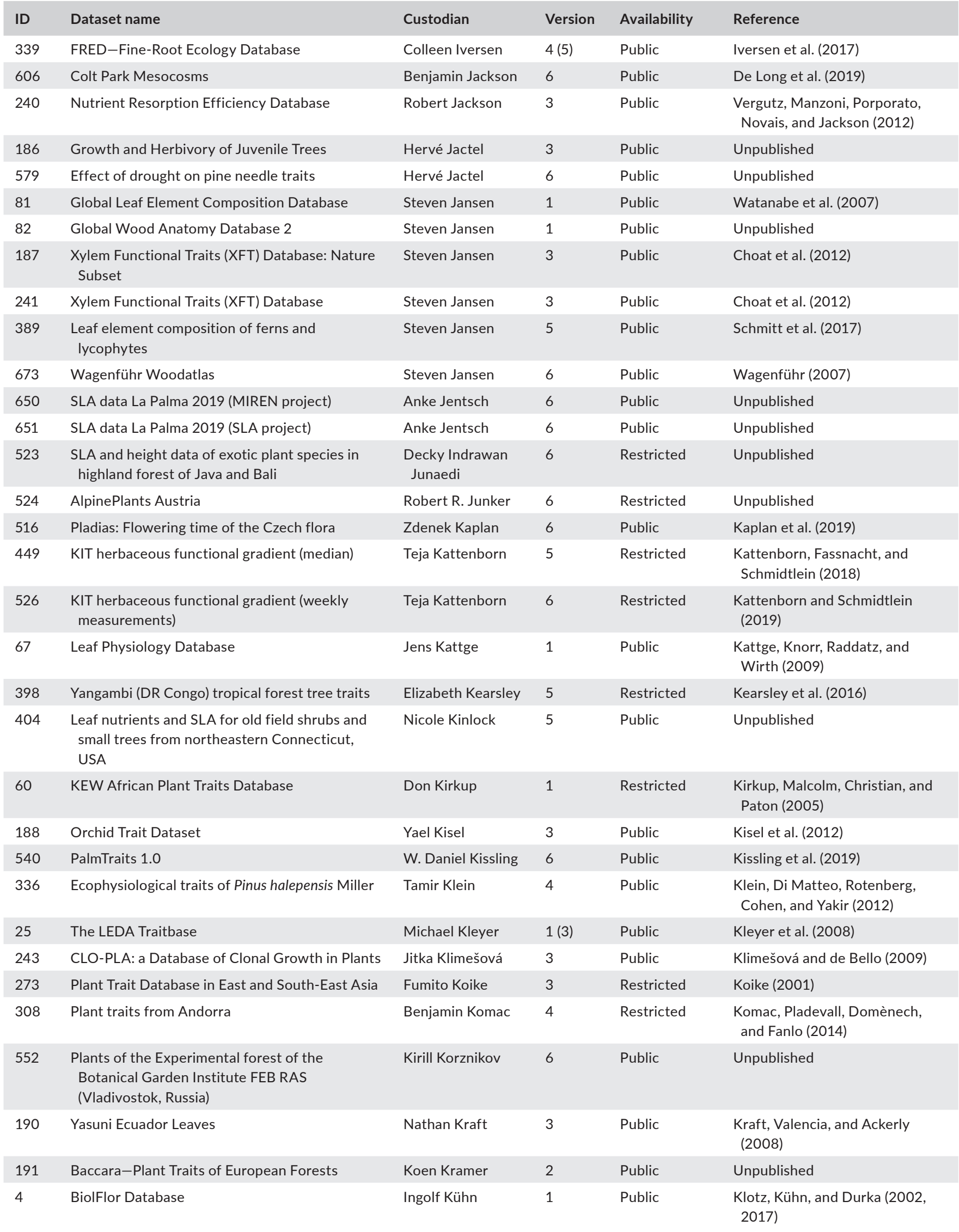


TABLE A1 (Continued)

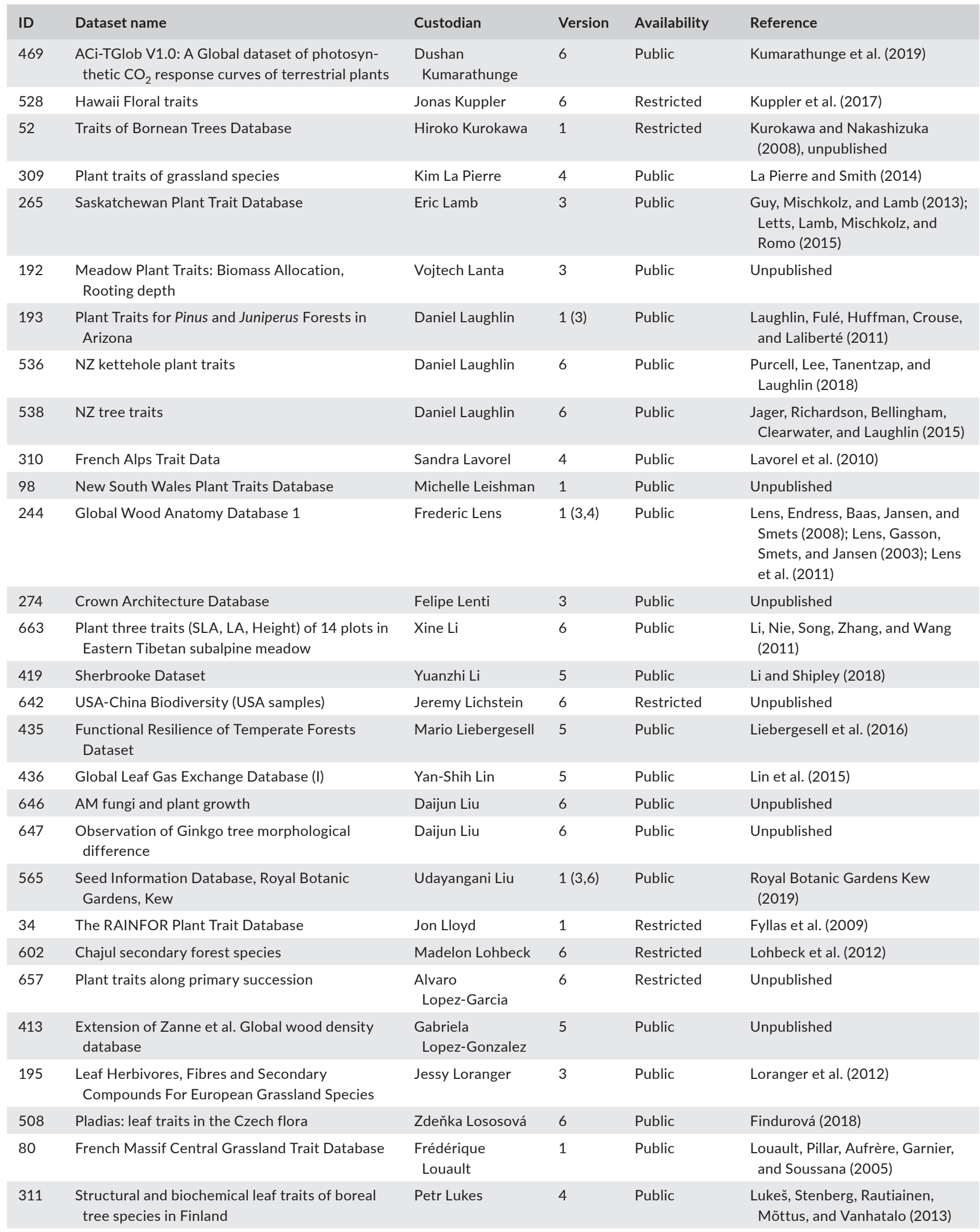


TABLE A1 (Continued)

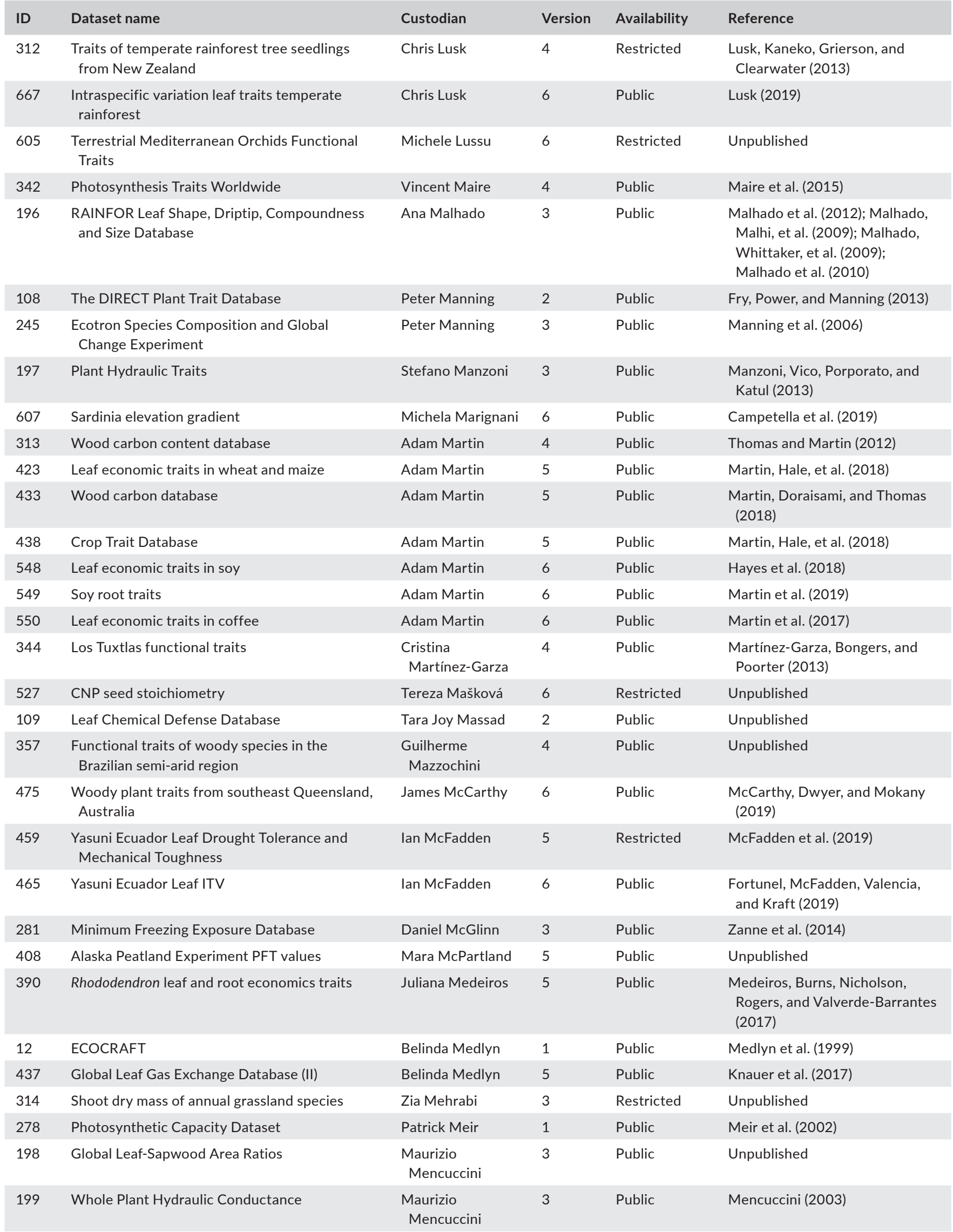


TABLE A1 (Continued)

\begin{tabular}{|c|c|c|c|c|c|}
\hline ID & Dataset name & Custodian & Version & Availability & Reference \\
\hline 315 & $\begin{array}{l}\text { Leaf traits for Picea glauca and Pinus sylvestris on } \\
\text { University of Calgary (Canada) campus }\end{array}$ & Sean Michaletz & 4 & Public & Michaletz and Johnson (2006) \\
\hline 200 & Altitudinal Vicariants Spain & Ruben Milla & 3 & Public & Milla and Reich (2011) \\
\hline 415 & Ozark glade grassland plants & Jesse Miller & 5 & Public & $\begin{array}{l}\text { Miller, Ives, Harrison, and } \\
\text { Damschen (2017) }\end{array}$ \\
\hline 247 & $\begin{array}{l}\text { Traits of Halophytic Species in } \\
\text { North-West-Germany }\end{array}$ & Vanessa Minden & 3 & Restricted & Minden and Kleyer (2011) \\
\hline 456 & $\begin{array}{l}\text { Trait-responses of Impatiens species to light and } \\
\text { nutrients }\end{array}$ & Vanessa Minden & 5 & Restricted & Minden and Gorschlüter (2016) \\
\hline 457 & Antibiotics-effects on plant traits & Vanessa Minden & 5 & Restricted & $\begin{array}{l}\text { Minden, Deloy, Volkert, } \\
\text { Leonhardt, and Pufal (2017) }\end{array}$ \\
\hline 458 & Antibiotics-effects on plant elements & Vanessa Minden & 5 & Restricted & $\begin{array}{l}\text { Minden, Schnetger, Pufal, and } \\
\text { Leonhardt (2018) }\end{array}$ \\
\hline 518 & Plant traits along NPK gradients & Vanessa Minden & 6 & Restricted & $\begin{array}{l}\text { Minden and Olde Venterink } \\
\text { (2019) }\end{array}$ \\
\hline 317 & $\begin{array}{l}\text { Traits of Hypochaeris radicata under shade and } \\
\text { drought conditions }\end{array}$ & Rachel Mitchell & 4 & Public & Unpublished \\
\hline 202 & Traits from the Wildfire Project & Marco Moretti & 3 & Public & Moretti and Legg (2009) \\
\hline 307 & Hokkaido leaf traits & Akira Mori & 4 & Restricted & Mori et al. (2015) \\
\hline 537 & Hokkaido plant traits 2 & Akira Mori & 6 & Restricted & Unpublished \\
\hline 555 & Teshio grassland plant traits & Akira Mori & 6 & Restricted & Unpublished \\
\hline 556 & Utanai forest tree traits & Akira Mori & 6 & Restricted & Unpublished \\
\hline 557 & Kuujjuarapik-Whapmagoostui & Akira Mori & 6 & Restricted & Unpublished \\
\hline 655 & Functional Flowering Plant Traits & Jane Morrison & 6 & Public & Unpublished \\
\hline 318 & $\begin{array}{l}\text { Leaf traits related to mesophyll conductance in } \\
\text { wild relatives of tomato (Solanum lycopersicon) }\end{array}$ & Christopher Muir & 3 & Public & $\begin{array}{l}\text { Muir, Hangarter, Moyle, and } \\
\text { Davis (2013) }\end{array}$ \\
\hline 648 & LEVEG-UFRGS & Sandra Müller & 6 & Restricted & Unpublished \\
\hline 353 & Old fields of Eastern US (Siefert Data) & Luka Negoita & 4 & Public & Siefert, Fridley, and Ritchie (2014) \\
\hline 484 & $\begin{array}{l}\text { Larix occidentalis branch section, specific leaf } \\
\text { area and dry mass }\end{array}$ & Andrew Nelson & 6 & Restricted & Williams and Nelson (2018) \\
\hline 409 & Seed trait data from Neuschulz et al. 2016 & $\begin{array}{l}\text { Eike Lena } \\
\text { Neuschulz }\end{array}$ & 5 & Restricted & Neuschulz et al. (2016) \\
\hline 560 & Fruit Traits Ecuador & $\begin{array}{l}\text { Eike Lena } \\
\text { Neuschulz }\end{array}$ & 6 & Public & Quitián et al. (2018) \\
\hline 49 & Tree Tolerance Database & Ülo Niinemets & 1 & Restricted & Niinemets and Valladares (2006) \\
\hline
\end{tabular}


TABLE A1 (Continued)

\begin{tabular}{|c|c|c|c|c|c|}
\hline ID & Dataset name & Custodian & Version & Availability & Reference \\
\hline 453 & European North Russia & $\begin{array}{l}\text { Alexander } \\
\text { Novakovskiy }\end{array}$ & 5 & Public & $\begin{array}{l}\text { Dalke, Novakovskiy, Maslova, } \\
\text { and Dubrovskiy (2018) }\end{array}$ \\
\hline 203 & Plant Traits from Romania & Kinga Öllerer & 3 & Public & $\begin{array}{l}\text { Ciocârlan (2009), Sanda, Bita- } \\
\text { Nicolae and Barabas (2003) }\end{array}$ \\
\hline 635 & Olson PNAS 2018 & Mark E. Olson & 6 & Restricted & Olson, Soriano, et al. (2018) \\
\hline 637 & Rosell Olson Self Non self VD scaling & Mark E. Olson & 6 & Restricted & Rosell and Olson (2014) \\
\hline 638 & Olson et al. AnnBot 2018 Corners Rules & Mark E. Olson & 6 & Restricted & $\begin{array}{l}\text { Olson, Rosell, Zamora Muñoz, } \\
\text { and Castorena (2018) }\end{array}$ \\
\hline 410 & Onoda 2017 leaf traits dataset & Yusuke Onoda & 5 & Public & Onoda et al. (2017) \\
\hline 319 & $\begin{array}{l}\text { Plant Traits from Fynbos Forests in the Cape } \\
\text { Region }\end{array}$ & Renske Onstein & 4 & Public & $\begin{array}{l}\text { Onstein, Carter, Xing, and Linder } \\
\text { (2014) }\end{array}$ \\
\hline 88 & The Netherlands Plant Traits Database & Jenny Ordonez & 1 & Public & Ordoñez et al. (2010) \\
\hline 520 & Pinnacle Reserve, ACT & $\begin{array}{l}\text { Andrew } \\
\text { OReilly-Nugent }\end{array}$ & 6 & Public & OReilly-Nugent et al. (2019) \\
\hline 604 & $\begin{array}{l}\text { Absorptive root morphological traits of boreal } \\
\text { and hemi-boreal alder, birch and spruce forests }\end{array}$ & Ivika Ostonen & 6 & Public & $\begin{array}{l}\text { Ostonen et al. (2013); Ostonen, } \\
\text { Tedersoo, Suvi, and Lõhmus } \\
\text { (2009) }\end{array}$ \\
\hline 464 & Leaf traits of selected trees and Liana traits & Vivek Pandi & 6 & Public & Unpublished \\
\hline 623 & Fagus sylvatica Paggeo Greece & $\begin{array}{l}\text { Aristotelis C. } \\
\text { Papageorgiou }\end{array}$ & 6 & Public & Unpublished \\
\hline 320 & Grassland Plant Trait Database & Meelis Pärtel & $3(4)$ & Public & Takkis (2014) \\
\hline 27 & BROT Plant Trait Database & Juli Pausas & 1 & Public & Paula et al. (2009) \\
\hline 440 & P50R-A global P50 and Resprouting Database & Juli Pausas & 5 & Public & Pausas et al. (2015) \\
\hline 441 & BBB-A global Belowground Bud Bank database & Juli Pausas & 5 & Public & $\begin{array}{l}\text { Pausas, Lamont, Paula, Appezzato- } \\
\text { da-Glória, and Fidelis (2018) }\end{array}$ \\
\hline 474 & BROT 2.0 & Juli Pausas & 6 & Public & Tavşanoğlu and Pausas (2018) \\
\hline 270 & Plant Traits of Acidic Grasslands in Central Spain & Begoña Peco & 3 & Public & $\begin{array}{l}\text { Peco, de Pablos, Traba, and } \\
\text { Levassor (2005) }\end{array}$ \\
\hline 91 & Catalonian Mediterranean Forest Trait Database & Josep Peñuelas & 1 & Restricted & Ogaya and Peñuelas (2003) \\
\hline 114 & Hawaiian Leaf Traits Database & Josep Peñuelas & 2 & Restricted & Peñuelas et al. (2009) \\
\hline 131 & $\begin{array}{l}\text { Catalonian Mediterranean Shrubland Trait } \\
\text { Database }\end{array}$ & Josep Peñuelas & 1 & Restricted & Unpublished \\
\hline 493 & Weiqi-Sardans-Peñuelas China plants & Josep Peñuelas & 6 & Restricted & Unpublished \\
\hline 496 & Garraf-Peñuelas & Josep Peñuelas & 6 & Restricted & Peñuelas et al. (2017) \\
\hline
\end{tabular}


TABLE A1 (Continued)

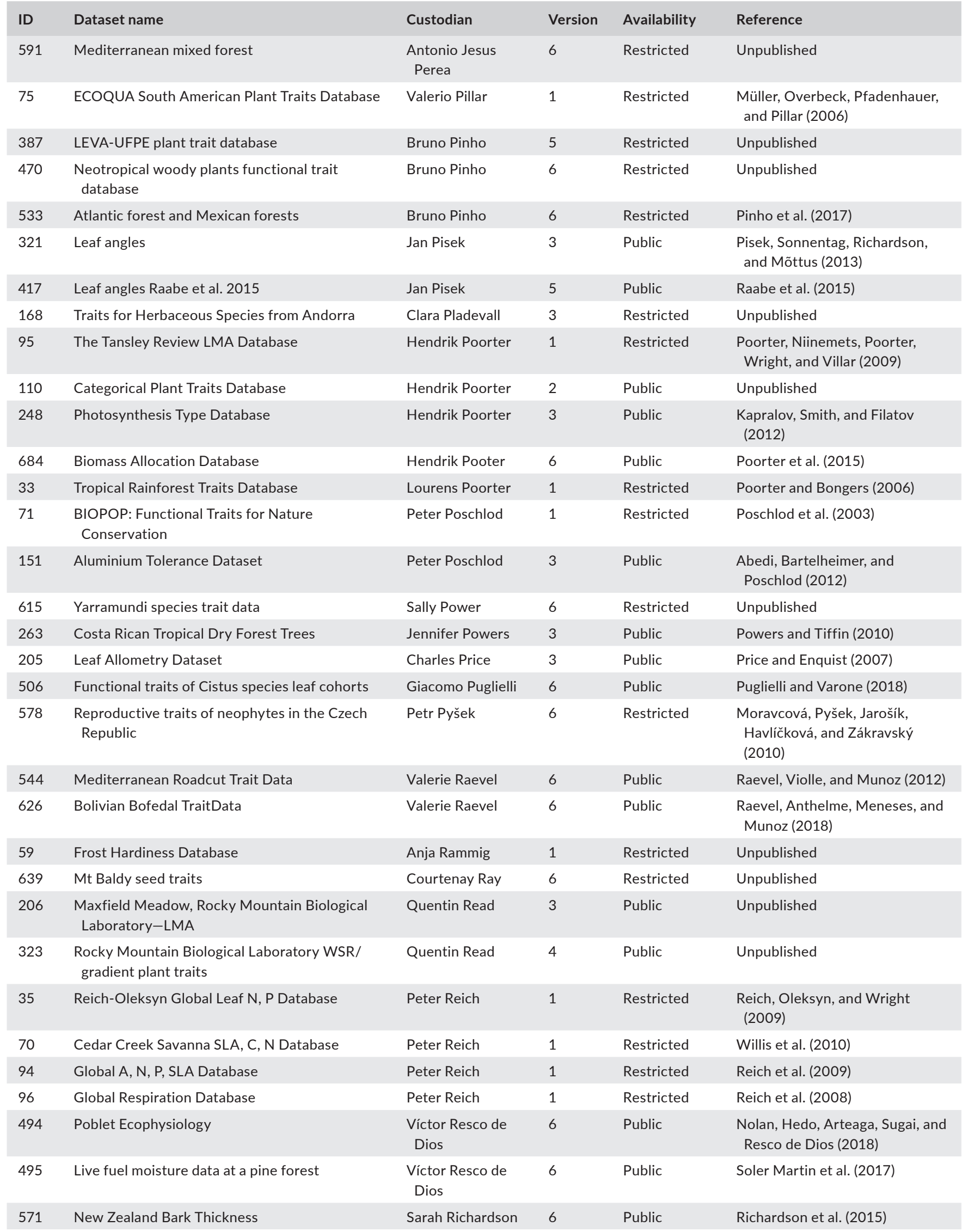


TABLE A1 (Continued)

\begin{tabular}{|c|c|c|c|c|c|}
\hline ID & Dataset name & Custodian & Version & Availability & Reference \\
\hline 573 & $\begin{array}{l}\text { New Zealand Alpine Granite Leaf Nutrient } \\
\text { Concentrations }\end{array}$ & Sarah Richardson & 6 & Public & Richardson et al. (2012) \\
\hline 343 & Sphagnum tissue CNP & Bjorn Robroek & 4 & Public & Unpublished \\
\hline 620 & Leaf and flower pressure volume curve data & Adam Roddy & 6 & Public & $\begin{array}{l}\text { Roddy, Jiang, Cao, Simonin, and } \\
\text { Brodersen (2019) }\end{array}$ \\
\hline 207 & $\begin{array}{l}\text { Herbaceous Plants Traits From Southern } \\
\text { Germany }\end{array}$ & $\begin{array}{l}\text { Christine } \\
\text { Roemermann }\end{array}$ & 3 & Public & Unpublished \\
\hline 400 & $\begin{array}{l}\text { Leaf Mass Area, Leaf Carbon and Nitrogen } \\
\text { Content from Barrow, Alaska }\end{array}$ & Alistair Rogers & 5 & Public & $\begin{array}{l}\text { Rogers, Serbin, Ely, Sloan, and } \\
\text { Wullschleger (2017) }\end{array}$ \\
\hline 402 & $\begin{array}{l}\text { Arctic Photosynthetic parameter Vcmax } \\
\text { Estimated Using the 1-Point Method }\end{array}$ & Alistair Rogers & 5 & Public & Rogers et al. (2017) \\
\hline 325 & Rollinson DBH & Emily Rollinson & 4 & Public & Unpublished \\
\hline 326 & Leaf nutrient concentrations & $\begin{array}{l}\text { Victor Rolo } \\
\text { Romero }\end{array}$ & 3 & Public & $\begin{array}{l}\text { Rolo, López-Díaz, and Moreno } \\
\text { (2012) }\end{array}$ \\
\hline 396 & Rehabilitating Coastal dune forest & $\begin{array}{l}\text { Victor Rolo } \\
\text { Romero }\end{array}$ & 5 & Restricted & $\begin{array}{l}\text { Rolo, Olivier, and van Aarde } \\
\text { (2016) }\end{array}$ \\
\hline 590 & Leaf Traits of Aquatic Plants & Dina Ronzhina & 6 & Public & Ronzhina and P'Yankov (2001) \\
\hline 589 & Jena Experiment Traits & $\begin{array}{l}\text { Christiane } \\
\text { Roscher }\end{array}$ & 6 & Public & $\begin{array}{l}\text { Gubsch et al. (2011); Lipowsky } \\
\text { et al. (2015); Roscher, Schmid, } \\
\text { Buchmann, Weigelt, and } \\
\text { Schulze (2011) }\end{array}$ \\
\hline 633 & $\begin{array}{l}\text { Bark Wood traits New Phytol } 2014 \text { and } \\
\text { Oecologia } 2015\end{array}$ & Julieta Rosell & 6 & Restricted & $\begin{array}{l}\text { Rosell, Gleason, Méndez-Alonzo, } \\
\text { Chang, and Westoby (2013) }\end{array}$ \\
\hline 519 & Swiss National Park, Engadine & Christian Rossi & 6 & Restricted & Rossi (2017) \\
\hline 208 & $\begin{array}{l}\text { Response of Tree Growth to Light and Size, } \\
\text { Barro Colorado Island, Panama }\end{array}$ & Nadja Rüger & 3 & Public & $\begin{array}{l}\text { Rüger, Berger, Hubbell, } \\
\text { Vieilledent, and Condit (2011) }\end{array}$ \\
\hline 283 & $\begin{array}{l}\text { Response of Tree Mortality to Light, Size and } \\
\text { Past Growth, Barro Colorado Island, Panama }\end{array}$ & Nadja Rüger & 3 & Public & $\begin{array}{l}\text { Rüger, Huth, Hubbell, and Condit } \\
\text { (2011) }\end{array}$ \\
\hline 284 & $\begin{array}{l}\text { Response of Tree Recruitment to Light, Barro } \\
\text { Colorado Island, Panama }\end{array}$ & Nadja Rüger & 3 & Public & $\begin{array}{l}\text { Rüger, Huth, Hubbell, and Condit } \\
\text { (2009) }\end{array}$ \\
\hline 672 & DISEQU-ALP & Sabine Rumpf & 6 & Public & Rumpf et al. (2018) \\
\hline 111 & Leaf and Whole-Plant Traits Database & Lawren Sack & 2 & Restricted & $\begin{array}{l}\text { Nakahashi, Frole, and Sack } \\
(2005)\end{array}$ \\
\hline 675 & Salguero-Gomez Cistus albidus 2019 & $\begin{array}{l}\text { Rob } \\
\text { Salguero-Gomez }\end{array}$ & 6 & Public & Unpublished \\
\hline 249 & California Coastal Grassland Database & Brody Sandel & 3 & Public & Sandel, Corbin, and Krupa (2011) \\
\hline 543 & Functional traits related to flammability & $\begin{array}{l}\text { Carolina } \\
\text { Santacruz }\end{array}$ & 6 & Restricted & Unpublished \\
\hline 407 & $\begin{array}{l}\text { Leaf nutrient concentrations from Scalon et al. } \\
2017\end{array}$ & Marina Scalon & 5 & Public & Scalon et al. (2017) \\
\hline
\end{tabular}


TABLE A1 (Continued)

\begin{tabular}{|c|c|c|c|c|c|}
\hline ID & Dataset name & Custodian & Version & Availability & Reference \\
\hline 211 & BIOTREE Trait Shade Experiment & $\begin{array}{l}\text { Michael } \\
\text { Scherer-Lorenzen }\end{array}$ & 3 & Public & $\begin{array}{l}\text { Scherer-Lorenzen, Schulze, Don, } \\
\text { Schumacher, and Weller (2007) }\end{array}$ \\
\hline 350 & Trait Data for African Plants-A Photo Guide & Marco Schmidt & 4 & Public & $\begin{array}{l}\text { Dressler, Schmidt, and Zizka } \\
\text { (2014) }\end{array}$ \\
\hline 395 & $\begin{array}{l}\text { Senckenberg leaf venation data of West African } \\
\text { Plants }\end{array}$ & Julio Schneider & 5 & Restricted & Unpublished \\
\hline 584 & $\begin{array}{l}\text { Traits of Woody Plants in Hluhluwe-iMfolozi } \\
\text { Park, South Africa }\end{array}$ & $\begin{array}{l}\text { Simon D. } \\
\text { Schowanek }\end{array}$ & 6 & Restricted & Unpublished \\
\hline 251 & The Xylem/Phloem Database & $\begin{array}{l}\text { Fritz } \\
\text { Schweingruber }\end{array}$ & 3 & Public & $\begin{array}{l}\text { Schweingruber and Landolt } \\
(2005)\end{array}$ \\
\hline 356 & $\begin{array}{l}\text { Aboveground morphological traits of grassland } \\
\text { species }\end{array}$ & $\begin{array}{l}\text { Marina } \\
\text { Semchenko }\end{array}$ & 4 & Restricted & $\begin{array}{l}\text { Abakumova, Zobel, Lepik, and } \\
\text { Semchenko (2016) }\end{array}$ \\
\hline 351 & Miombo tree species-SLA, leaf and seed size & Colleen Seymour & 4 & Public & $\begin{array}{l}\text { Joseph, Seymour, Cumming, } \\
\text { Cumming, and Mahlangu (2014) }\end{array}$ \\
\hline 352 & Miombo tree species-Leaf nutrients & Colleen Seymour & 4 & Public & Seymour et al. (2014) \\
\hline 485 & Catimbau National Park, Brazil & Julia Sfair & 6 & Restricted & $\begin{array}{l}\text { Sfair, de Bello, de França, } \\
\text { Baldauf, and Tabarelli (2018) }\end{array}$ \\
\hline 212 & $\begin{array}{l}\text { Herbs Water Relations on Soil Moisture } \\
\text { Gradients }\end{array}$ & Serge Sheremetev & 3 & Public & $\begin{array}{l}\text { Sheremetiev and Chebotareva } \\
\text { (2018) }\end{array}$ \\
\hline 412 & The Global Leaf Traits Database & Serge Sheremetev & 5 & Public & Unpublished \\
\hline 471 & Species Growth Forms (Angiosperms)-Update 9 & Serge Sheremetev & 6 & Public & $\begin{array}{l}\text { Sheremetiev and Chebotareva } \\
\text { (2018) }\end{array}$ \\
\hline 483 & $\begin{array}{l}\text { A Geological Age of an Angiosperm Genera and } \\
\text { Families }\end{array}$ & Serge Sheremetev & 6 & Public & $\begin{array}{l}\text { Sheremetiev and Chebotareva } \\
\text { (2018) }\end{array}$ \\
\hline 99 & Tropical Traits from West Java Database & Satomi Shiodera & 1 & Public & $\begin{array}{l}\text { Shiodera, Rahajoe, and Kohyama } \\
\text { (2008) }\end{array}$ \\
\hline 50 & Leaf and Whole Plant Traits Database & Bill Shipley & 1 & Public & Shipley (2002) \\
\hline 252 & Leaf Structure and Chemistry & Bill Shipley & 3 & Public & Auger and Shipley (2012) \\
\hline 608 & $\begin{array}{l}\text { Traits of understory plants of western Canadian } \\
\text { forest }\end{array}$ & $\begin{array}{l}\text { Tanvir Ahmed } \\
\text { Shovon }\end{array}$ & 6 & Restricted & Shovon et al. (2019) \\
\hline 616 & Div Resource Pot Experiment & Alrun Siebenkäs & 6 & Restricted & $\begin{array}{l}\text { Siebenkäs, Schumacher, and } \\
\text { Roscher (2015) }\end{array}$ \\
\hline 133 & New York Old Field Plant Traits Database & Andrew Siefert & 2 & Restricted & Siefert (2011) \\
\hline 327 & Eastern US Old Field Plant Traits Database & Andrew Siefert & 4 & Public & Siefert et al. (2014) \\
\hline
\end{tabular}


TABLE A1 (Continued)

\begin{tabular}{|c|c|c|c|c|c|}
\hline ID & Dataset name & Custodian & Version & Availability & Reference \\
\hline 568 & $\begin{array}{l}\text { LABDENDRO Brazilian Subtropical Forest Traits } \\
\text { Database [Dataset III] }\end{array}$ & Ana Carolina Silva & 6 & Restricted & Soboleski et al. (2017) \\
\hline 534 & Silva el al. 2019 & Vasco Silva & 6 & Restricted & Silva, Catry, et al. (2019) \\
\hline 358 & Leaf Respiration Acclimation in Panama & Martijn Slot & 4 & Public & $\begin{array}{l}\text { Slot, Rey-Sánchez, Winter, and } \\
\text { Kitajima (2014) }\end{array}$ \\
\hline 385 & Photosynthesis Temperature Response Panama & Martijn Slot & 5 & Restricted & Slot and Winter (2017) \\
\hline 424 & $\begin{array}{l}\text { LCE: Leaf carbon exchange dataset for tropical, } \\
\text { temperate, and boreal species of North and } \\
\text { Central America }\end{array}$ & Nick Smith & 5 & Public & Smith and Dukes (2017) \\
\hline 328 & Root Traits of Grassland Species & $\begin{array}{l}\text { Stuart William } \\
\text { Smith }\end{array}$ & 4 & Public & $\begin{array}{l}\text { Smith, Woodin, Pakeman, } \\
\text { Johnson, and van der Wal } \\
\text { (2014) }\end{array}$ \\
\hline 558 & Sonnier and Boughton ABS & Gregory Sonnier & 6 & Public & Unpublished \\
\hline 454 & Leaf traits from ECOSHRUB Dovrefjell Norway & $\begin{array}{l}\text { Mia Vedel } \\
\text { Sørensen }\end{array}$ & 5 & Public & Unpublished \\
\hline 77 & FAPESP Brazil Rainforest Database & Enio Sosinski & 1 (3) & Restricted & Unpublished \\
\hline 256 & Niwot Alpine Plant Traits & Marko Spasojevic & 3 & Public & Spasojevic and Suding (2012) \\
\hline 418 & Ozark Tree leaf traits & Marko Spasojevic & 5 & Public & $\begin{array}{l}\text { Spasojevic, Turner, and Myers } \\
\text { (2016) }\end{array}$ \\
\hline 674 & $\begin{array}{l}\text { Staples et al. Australian Reforestation Tree } \\
\text { Database }\end{array}$ & Timothy Staples & 6 & Public & $\begin{array}{l}\text { Staples, Dwyer, England, and } \\
\text { Mayfield (2019) }\end{array}$ \\
\hline 547 & $\begin{array}{l}\text { Traits of Alpine species in GLORIA regions } \\
\text { Hochschwab, Schrankogel, Majella and Lefka } \\
\text { Ori }\end{array}$ & Klaus Steinbauer & 6 & Restricted & Unpublished \\
\hline 364 & Plant species high elevation dataset & Christien Steyn & 4 & Public & $\begin{array}{l}\text { Steyn, Greve, Robertson, Kalwij, } \\
\text { and le Roux (2016) }\end{array}$ \\
\hline 577 & Marion Island Fine Scale & Tanya Strydom & 6 & Restricted & Unpublished \\
\hline 610 & $\begin{array}{l}\text { Ash Free Dry Mass of Ceratophyllum } \\
\text { submersum }\end{array}$ & Ivana Svitkova & 6 & Restricted & Unpublished \\
\hline 51 & Tropical Plant Traits From Borneo Database & Emily Swaine & 1 & Public & Swaine (2007) \\
\hline 214 & $\begin{array}{l}\text { Maximum Height of Chinese Tree Species (from } \\
\text { Silva Sinica) }\end{array}$ & Nathan Swenson & 3 & Public & Zheng (1983) \\
\hline 288 & CTFS Luquillo Forest Dynamics Plot & Nathan Swenson & 4 & Public & $\begin{array}{l}\text { Swenson, Anglada-Cordero, and } \\
\text { Barone (2010) }\end{array}$ \\
\hline
\end{tabular}

(Continues) 
TABLE A1 (Continued)

\begin{tabular}{|c|c|c|c|c|c|}
\hline ID & Dataset name & Custodian & Version & Availability & Reference \\
\hline 345 & CIRAD Selmet Tree LNC Sahel & $\begin{array}{l}\text { Simon } \\
\text { Taugourdeau }\end{array}$ & 4 & Public & Unpublished \\
\hline 451 & $\begin{array}{l}\text { NodDB-A global database of plants with root- } \\
\text { symbiotic nitrogen fixation }\end{array}$ & Leho Tedersoo & 5 & Public & Tedersoo et al. (2018) \\
\hline 625 & Tng et al. 2013 Traits & David Tng & 6 & Public & Tng et al. (2013) \\
\hline 575 & Myricaria germanica & Sitzia Tommaso & 6 & Restricted & $\begin{array}{l}\text { Sitzia, Michielon, lacopino, and } \\
\text { Kotze (2016) }\end{array}$ \\
\hline 665 & Species patch metrics & Sitzia Tommaso & 6 & Public & $\begin{array}{l}\text { Sitzia, Dainese, Krüsi, and } \\
\text { McCollin (2017) }\end{array}$ \\
\hline 338 & Leaf Traits and Seed Mass of Cover Crops & Hélène Tribouillois & 4 & Public & Tribouillois et al. (2015) \\
\hline 598 & $\begin{array}{l}\text { Soft traits of the Northern Swan Coastal Plain } \\
\text { and Geraldton Sandplain kwongan vegetation, } \\
\text { Western Australia }\end{array}$ & James Tsakalos & 6 & Restricted & Unpublished \\
\hline 685 & Tree and Forest Biomass Distribution & Vladimir Usoltsev & 6 & Public & Usoltsev (2010) \\
\hline 216 & Traits for Common Grasses and Herbs in Spain & $\begin{array}{l}\text { Fernando } \\
\text { Valladares }\end{array}$ & 3 & Public & Unpublished \\
\hline 56 & Wetland Dunes Database & $\begin{array}{l}\text { Peter van } \\
\text { Bodegom }\end{array}$ & 1 & Restricted & $\begin{array}{l}\text { van Bodegom, Sorrell, Oosthoek, } \\
\text { Bakke, and Aerts (2008) }\end{array}$ \\
\hline 617 & Forbs and grasses in North East Belgium & $\begin{array}{l}\text { Elisa Van } \\
\text { Cleemput }\end{array}$ & 6 & Public & $\begin{array}{l}\text { Van Cleemput, Roberts, Honnay, } \\
\text { and Somers (2019) }\end{array}$ \\
\hline 332 & $\begin{array}{l}\text { Photosynthetic parameters, respiration and leaf } \\
\text { traits of a Peruvian tropical montane cloud } \\
\text { forest }\end{array}$ & $\begin{array}{l}\text { Marjan van de } \\
\text { Weg }\end{array}$ & 4 & Public & $\begin{array}{l}\text { van de Weg, Meir, Grace, and } \\
\text { Ramos (2011) }\end{array}$ \\
\hline 333 & $\begin{array}{l}\text { LMA, leaf tissue density and N\&P content along } \\
\text { the Amazon-Andes gradient in Peru }\end{array}$ & $\begin{array}{l}\text { Marjan van de } \\
\text { Weg }\end{array}$ & 4 & Public & $\begin{array}{l}\text { van de Weg, Meir, Grace, and } \\
\text { Atkin (2009) }\end{array}$ \\
\hline 618 & Montane grassland Functional Traits & $\begin{array}{l}\text { Stephni van der } \\
\text { Merwe }\end{array}$ & 6 & Restricted & Unpublished \\
\hline 619 & Sub Antarctic tundra_Functional Traits & $\begin{array}{l}\text { Stephni van der } \\
\text { Merwe }\end{array}$ & 6 & Restricted & Unpublished \\
\hline 331 & $\begin{array}{l}\text { Traits of savannah trees in the Hluhluwe- } \\
\text { iMfolozi Game reserve, South Africa }\end{array}$ & Fons van der Plas & 4 & Public & $\begin{array}{l}\text { Van der Plas, Howison, Reinders, } \\
\text { Fokkema, and Olff (2013) }\end{array}$ \\
\hline 599 & Trait data Pibiri-Masha van der Sande & $\begin{array}{l}\text { Masha van der } \\
\text { Sande }\end{array}$ & 6 & Public & van der Sande et al. (2017) \\
\hline 562 & 1000 Seedweight & Mark van Kleunen & 6 & Public & $\begin{array}{l}\text { Chrobock, Kempel, Fischer, and } \\
\text { van Kleunen (2011) }\end{array}$ \\
\hline 563 & Germination & Mark van Kleunen & 6 & Public & Chrobock et al. (2011) \\
\hline 564 & Competition & Mark van Kleunen & 6 & Public & $\begin{array}{l}\text { Kempel, Chrobock, Fischer, Rohr, } \\
\text { and van Kleunen (2013) }\end{array}$ \\
\hline
\end{tabular}


TABLE A1 (Continued)

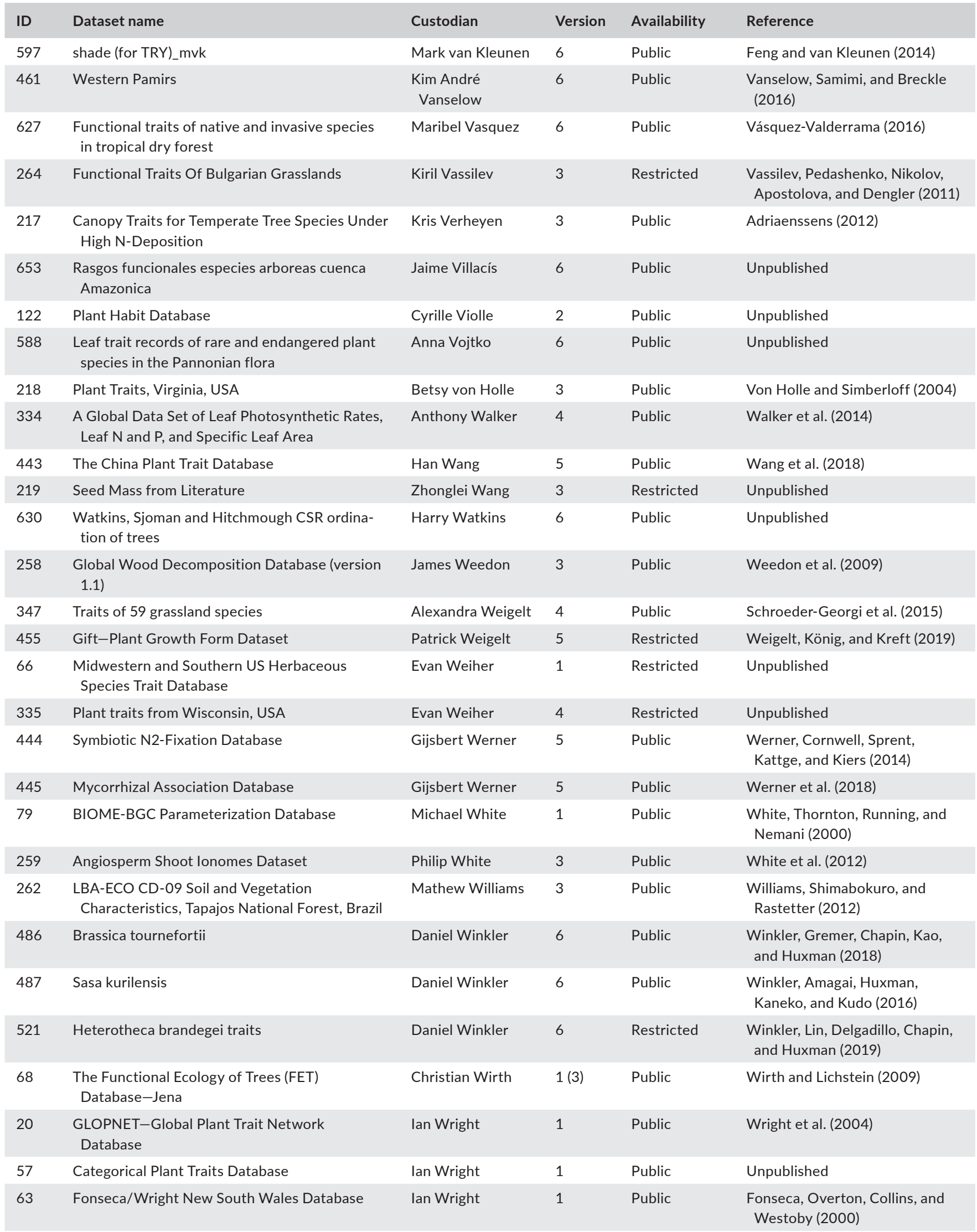


TABLE A1 (Continued)

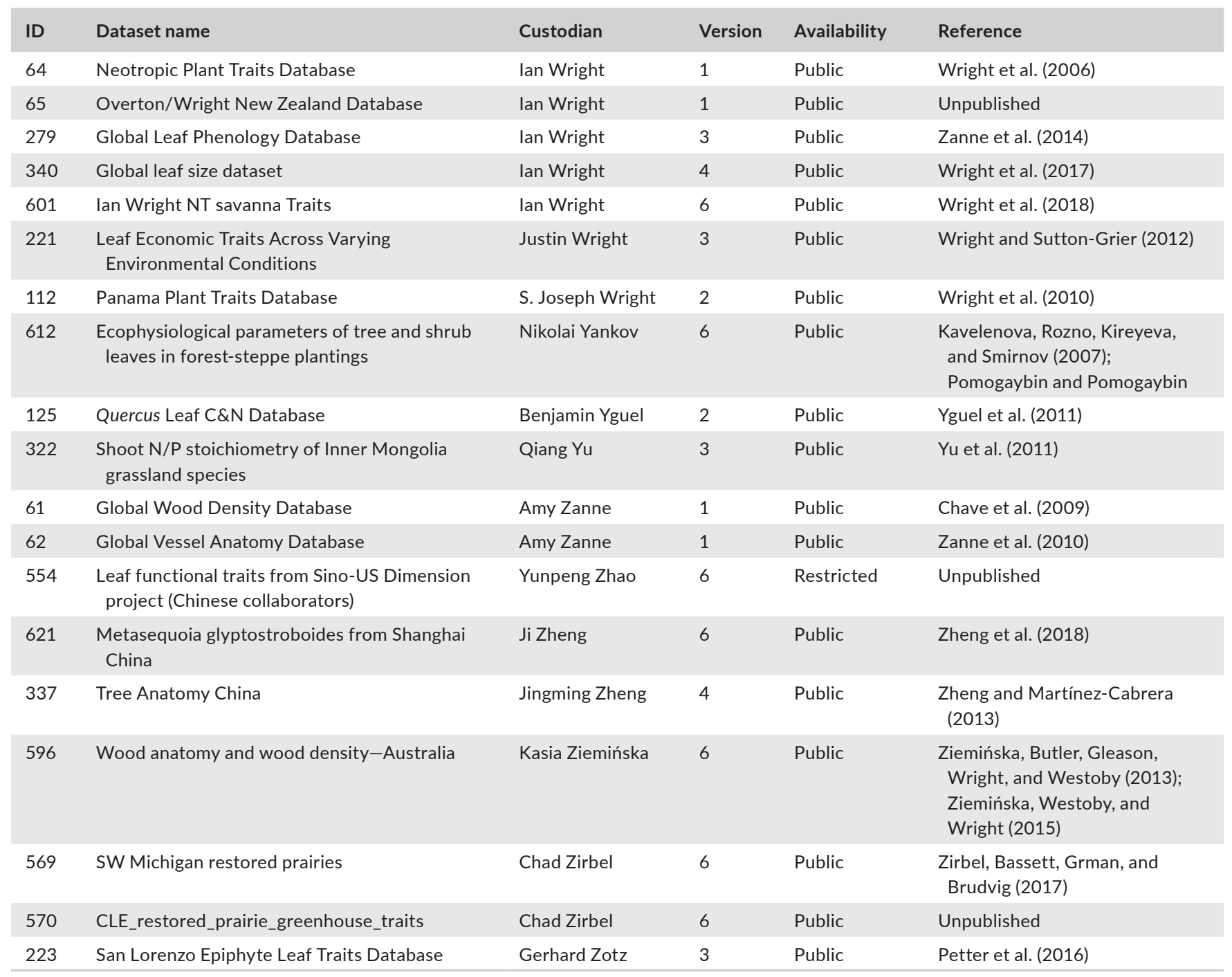

